UNMANNED AERIAL VEHICLE MAPPING FOR RESPONSIBLE LAND ADMINISTRATION

Elvira Claudia Stöcker 



\title{
UNMANNED AERIAL VEHICLE MAPPING FOR RESPONSIBLE LAND ADMINISTRATION
}

\author{
DISSERTATION
}

to obtain

the degree of doctor at the University of Twente, on the authority of the rector magnificus, prof.dr.ir. A. Veldkamp

on account of the decision of the Doctorate Board, to be publicly defended

on Thursday 9 December 2021 at 10.45 hrs

by

\section{Elvira Claudia Stöcker}

born on 19 January, 1989

in Dresden, Germany 
This dissertation has been approved by

Prof. mr. dr. ir. J.A. Zevenbergen, supervisor

Dr. M. N. Koeva, co-supervisor

Dr. F. C. Nex, co-supervisor

ITC dissertation number 407

ITC, P.O. Box 217, 7500 AE Enschede, The Netherlands

ISBN 978-90-365-5303-2

DOI $10.3990 / 1.9789036553032$

Cover designed by Claudia Stöcker and Job Duim

Printed by CTRL-P Hengelo

Copyright (C) 2021 by Claudia Stöcker

\section{UNIVERSITY OF TWENTE.}


Graduation committee:

\section{Chairman/Secretary}

Prof.dr. F.D. van der Meer University of Twente (ITC)

\section{Supervisor}

Prof.mr.dr.ir. J.A. Zevenbergen University of Twente (ITC)

\section{Co-supervisors}

Dr. M.N. Koeva

University of Twente (ITC)

Dr. F.C. Nex

University of Twente (ITC)

\section{Committee members}

Prof.mr.dr. M.A. Heldeweg

University of Twente (BMS)

Prof.dr.ir. C.H.J. Lemmen

University of Twente (ITC)

Prof.dr.ir. M.G. Vosselman

Prof.dr. G. Barnes

University of Twente (ITC)

Dr. J.B.K. Kiema

University of Florida

University of Nairobi 



\section{Acknowledgements}

Alles muss klein beginnen. Lass etwas Zeit verrinnen.

Es muss nur Kraft gewinnen. Und endlich ist es gross.

Gerhard Schöne (1988)

In accordance with the quote above, this $\mathrm{PhD}$ project also needed time to grow. Many experiences, conversations and encounters have significantly enriched this phase of my life and supported me in achieving this scientific, but also personal goal. This multifaceted PhD journey would not have been possible without the support and guidance of many people who made themselves available in numerous capacities.

I want to express my gratitude to my promoter Jaap Zevenbergen and my supervisors Mila Koeva and Francesco Nex. Jaap, thank you for your patient guidance and valuable critiques throughout the past years. I am grateful for the trust and freedom you gave me, always calling on the firm belief: she will manage. Mila, thank you for your constant research support, warmth, friendship and continuous encouragement to follow my way. Thank you, Francesco, for keeping me safe while working with UAVs. Your huge knowledge about this subject and your constant guidance truly helped me to achieve my goals. I do not want to miss the opportunity to thank Rohan Bennett and Markus Gerke, who supervised me early on in my PhD research. Thank you for inspiring me during the first year of this journey - it was so much fun to work with both of you. You taught me to reflect on my research findings critically and to take different perspectives. I am particularly grateful for our continued collaboration and constructive discussions, even after you left ITC. Jaap, Mila, Francesco, Rohan and Markus, you formed an outstanding complementary team, and I am very appreciative of your efforts.

I was lucky that my work was embedded in the $\mathrm{H} 2020$ its4land project (project number 687828). Rohan and Mila, thank you for your tremendous effort in running this multidisciplinary project, certainly a challenge on its own. I enjoyed the team spirit and learnt a lot about effective communication strategies. The project setting gave me numerous occasions to present and discuss my work internally. Sophie Crommelinck, Joep Crompvoets, Serene Ho, Angela Schwering, 
Malumbo Chipofya, Jan Sahib, Christian Timm, Berhanu Kefale Alemie, Cornelia Schmidt, thank you for all your critical questions during presentations and the constructive reviews of my project deliverables.

My fieldwork in Eastern Africa would not have been possible without the support of the its4land UAV pilot crew - the pilotees. First and foremost, Cornelia Schmidt, the first certified female UAV pilot in Rwanda. You are one of the most dedicated and eager persons I got to know during this PhD journey. Thank you, Dirk Ohnsorge, Placide Nkerabigwi, Peter Odwe, Robert Wayumba and Berhanu Kefale, for accomplishing the practical UAV training and for your constant efforts to seek flight approval. Kaspar and Connie, thanks for transforming from an excellent host in Kigali to my "Rwandan parents".

Without any doubt, the numerous fieldwork travel were the most exciting times of this PhD journey. I learned that it is good to make plans ahead. Still, it is even more important to stay flexible and spontaneous with creativity to find a viable solution for unforeseeable problems. Thank you all for bearing with me. Special thank goes to the staff of INES Ruhengeri, Technical University of Kenya, Charis UAS Ltd. and Tanzania Flying Labs for your field work assistance and the numerous flying hours for the sake of my research. The local support of the Rwanda Land Management and Use Authority and the Commission of Lands in Zanzibar ensured that my research did not get lost in theories but found answers to practical problems. Thanks to all the participants of the workshops, interview partners and respondents to my questionnaires all around the world.

I would also like to acknowledge my ITC colleagues for the nice time I had during my PhD. As a "sandwich" between EOS and PGM, I truly benefitted from both sides (not only from the two coffee breaks). Thanks to all EOS and PGM colleagues for their constant support and curiosity about my research, inspirational conversations and social activities. I thank Roeloff for his smile each morning, Watse and Robert for their support during the UAV flights, Benno and Job for the timely delivery of printing media for fieldwork and the cover design of this thesis, Chiel and Glen for keeping books about our finances. I am grateful for the constant support of the ITC library team, and especially to Marga who helped me with the data repository. A huge DANKE goes to Petra, who was always eager to help out. Thanks to my PhD colleagues Abishek, Alice, Andres, Anna, Andrea, Aulia, Eduardo, Fashuai, Fenna, Ieva, Kwabena, Mahmood, Phillipp, Shayan, Sophie, Rosa, Vera and Zaid, for your friendship, support and discussions at various moments. 
I am also grateful to the boulder enthusiasts in the Cube Bouldergym. This place slowly matured into our second home and was always an excellent spot to forget about work and focus on other physical and mental challenges. Especially the team challenge was a yearly highlight, thanks to the entire A+ team for the fun moments and joint boulder events.

Along my way, I met new friends who truly enriched this beautiful time in Enschede. Anna, thanks for being my mental mate in town and for all the joyful meetings with our little girls. Without you and Flo, we would never have found consensus on how to name our third child. Caroline, thank you for your close friendship and all your advice throughout my time in Enschede, and hopefully beyond. I enjoyed it so much to have you as a boulder mate and I cannot wait to go and do a multi-pitch with you. Desi for introducing us to the volleyball gang and the alternative festivals in Enschede. Shayan and Ieva, for all the nice events and the tasty Iranian food at your place. Maria and Nick for introducing us to the Dutch culture and for all the nice moments in the Cube. Ine for having such a great time together in Kenya and for hosting me in Leuven. I would also like to thank all my young and old friends from the Noorderkroon school.

Last but not least, to my friends and family back home in Dresden. I cannot imagine life without our lovely house community - I never felt home so quickly after moving. To my dear friends Sandra, Christoph, Odette, Elisabeth, Gabriel, Jule, Chrissi, Thomas, Juli, Paul, Kati and Flo who stayed very close even though we have not met very regularly during my time in Enschede. Thanks to Anne and Michael for being such great grandparents for our children and for helping Martin to watch the kids during the times I was on field work. To my sister Astrid who always supported me and stayed with us during the most pressing times. To my father for his assistance and patience. To my dear mother, for her love and encouragement - I wish you could still be with us.

Ida, Lotta and Jonathan, from the beginning of this encounter, you taught me patience and serenity. Being your mother and watching you grow is the most rewarding and beautiful thing in the world. Martin, thank you for daring to take this step with me, I remain with love and gratitude! 


\section{Table of Contents}

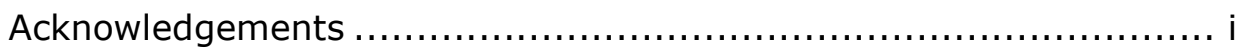

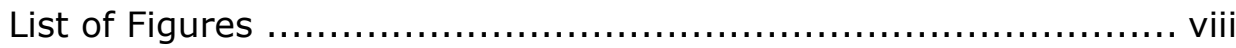

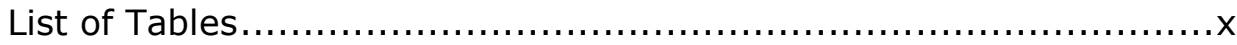

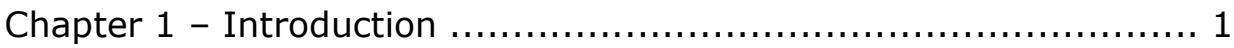

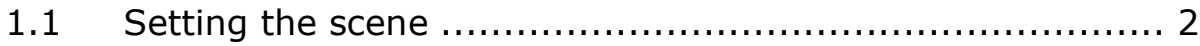

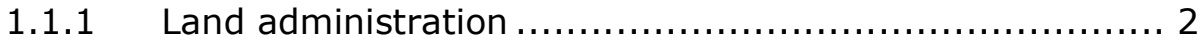

1.1 .2 Land information ........................................... 3

1.1.3 Alternative tools to collect land information ................. 4

1.1.4 Unmanned Aerial Vehicles ................................... 5

1.1.5 UAVs in land administration ............................... 7

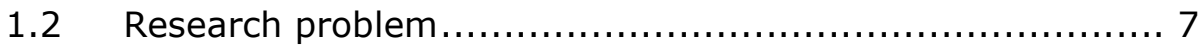

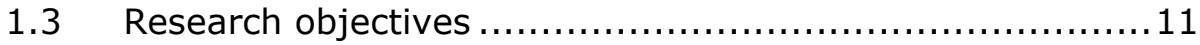

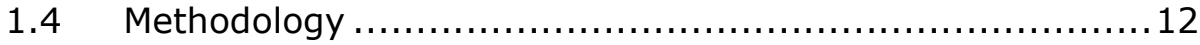

1.5 Outline of the thesis............................................ 14

Chapter 2 - UAV imagery, Land Data and User Needs .................17

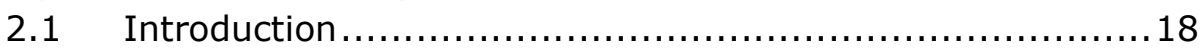

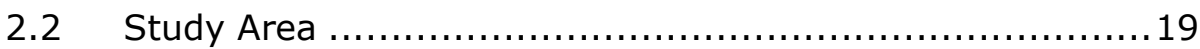

2.3 Material and Methods .......................................... 20

2.3.1 Needs Assessment......................................... 21

2.3.2 UAV Data Collection .........................................23

2.3.2.1 UAV Regulations in Rwanda ...............................23

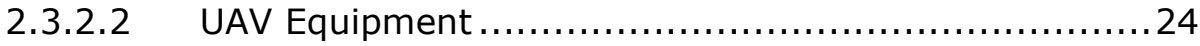

2.3.2.3 Ground Control Measurements ..........................27

2.3.2.4 Software and Hardware Requirements .................... 28

2.4 Results....................................................... 29

2.4.1 What Land Information do Rwandan Stakeholders Need?29

2.4.1.1 Government Stakeholders' Needs ...........................29

2.4.1.2 Local Government and Communities' Needs............... 31

2.4.1.3 Non-Government Stakeholders' Needs ..................... 31

2.4.2 What Data Quality can be Achieved with UAVs?............... 32

2.4.3 Can UAV Respond to the Needs Expressed by Different

Stakeholders? .................................................. 34

2.4.3.1 High Geometric Accuracy................................. 36

2.4.3.2 Provision of up-to-Date Data................................ 36

2.4.3.3 High Spatial and/or Temporal Resolution ...................37

2.4.3.4 High Level of Interpretability.............................. 38

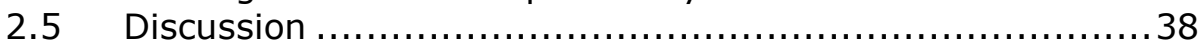

2.5.1 Opportunities of UAV Data Collection to Match Land Information Needs ...........................................39

2.5.2 Challenges of UAV Data Collection to Match Land

Information Needs...........................................40

2.5.3 Limitations of this Research and Future Work................4 41

2.6 Conclusion .................................................... 41 
Chapter 3 - Legal Considerations for Applying UAVs in Land

Administration ............................................................. 43

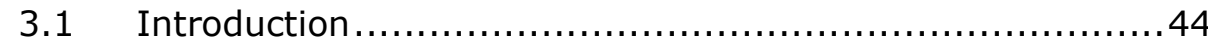

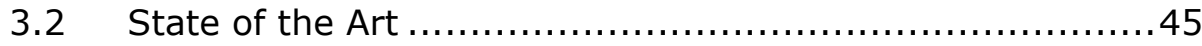

3.3 Data Sources and Methods ................................... 47

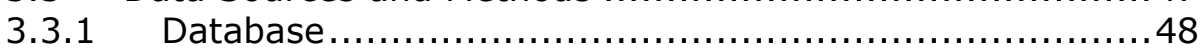

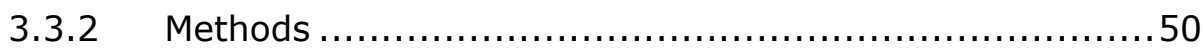

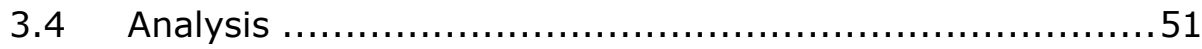

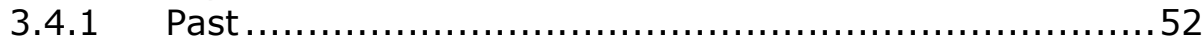

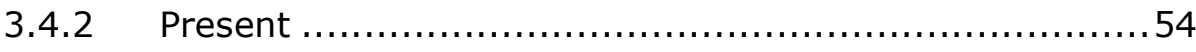

3.4.2.1 International Context ................................... 54

3.4.2.2 National Context ........................................ 56

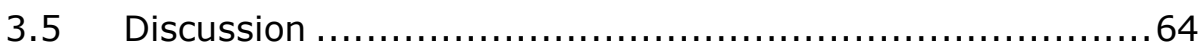

3.5.1 Future Trends and Challenges ............................6 64

3.5.2 Unmanned Aerial Vehicles (UAVs) in the Broader Context of Law and Technology.......................................69

3.5.3 Limitations of This Research .............................. 71

3.6 Conclusions .................................................. 72

Chapter 4 - Guidance for Optimal UAV Flight Configurations to Derive

High-Quality Orthophotos ........................................... 73

4.1 Introduction ....................................................... 74

4.2 Material and methods ......................................... 77

4.2.1 UAV and GNSS data collection.............................. 78

4.2.2 Estimating the impact of land cover on the number of automated tie points ....................................... 80

4.2.3 Estimating the impact of the number of GCPs on the final geometric accuracy ........................................ 82

4.2.4 Estimating the impact of different flight plans on the characteristics of extracted cadastral features ...............8 84

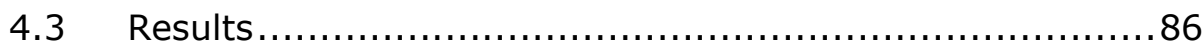

4.3.1 Image matching: image correspondences ...................86

4.3.2 Absolute accuracy: Checkpoint residuals in DSM and orthophotos ................................................ 88

4.3.3 Relative accuracy: Characteristics of automatically extracted cadastral features................................. 91

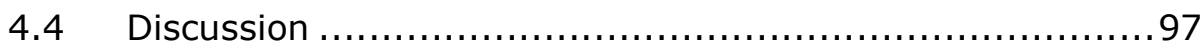

4.5 Conclusion ..................................................... 100

Chapter 5 - Scaling up UAVs in Land Administration: Towards the

Plateau of Productivity................................................. 103

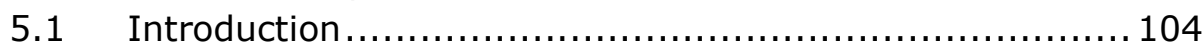

5.2 Background ............................................ 106

5.2.1 Framework for Effective Land Administration (FELA) ....107

5.2.2 Technology innovation and diffusion models ............... 108

5.2.3 Gartner's Hype Cycle ..................................... 110

5.3 Methods and Material ........................................ 111 
5.3.1 Combining the Hype Cycle with FELA $\ldots \ldots \ldots \ldots \ldots \ldots \ldots 112$

5.3.2 Data sources and analysis ................................. 114

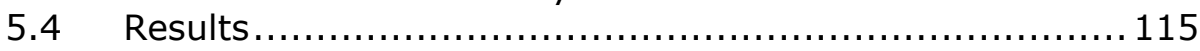

$5.4 .1 \quad$ Hype Cycle Analysis .......................................... 115

5.4.2 The relevance of FELA pathways during the progression of

UAV technology in land administration .................... 119

5.5 Discussion ..................................................... 126

5.6 Conclusion ..................................................... 128

Chapter 6 - Summary of the Main Findings and Reflections.......... 129

6.1 Introduction .................................................. 130

6.2 Conclusions per research objective ........................ 131

6.3 Conclusions on the main research objective ................. 134

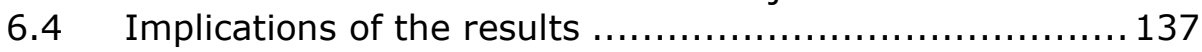

6.4.1 Contributions to scientific research ...................... 137

6.4.2 Contributions to land administration and policy ........... 138

6.4.3 Contributions to UAV practice and policy ..................... 139

6.5 Limitations of this work ..................................... 140

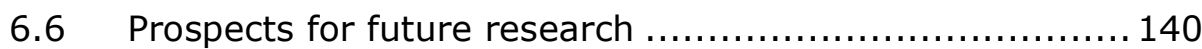

Bibliography......................................................... 142

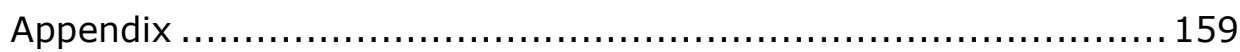

Appendix 1 - The its4land project ................................ 160

Appendix 2 - A practical excursus: Exemplifying a UAV-driven community mapping exercise to support the updating process of the Rwandan cadastre ............................................... 161

Appendix 3 - Overview of UAV regulations in selected countries 171

Summary .......................................................... 179

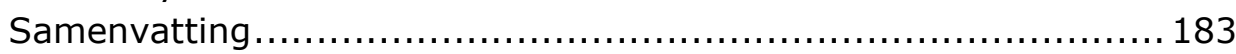

Zusammenfassung ...................................................... 187

Author's Biography .................................................... 191 


\section{List of Figures}

Figure 1.1:UAV-based mapping - requirements and data acquisition workflow

Figure 1.2: Conceptual framework and interrelationship of system

elements

Figure 1.3: Organization of this dissertation ......................... 14

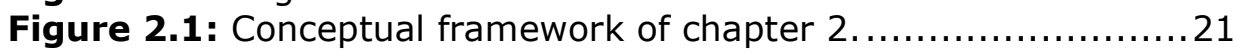

Figure 2.2: Overview of UAV data collection sites in Rwanda.........26

Figure 2.3: Measurement of reference points........................ 28

Figure 2.4: Overview of the generated orthomosaics and

$\mathrm{GCP} /$ checkpoint distribution.

Figure 2.5: Prioritized needs classified by the ability of UAV data to match stated needs ..................................................... 35

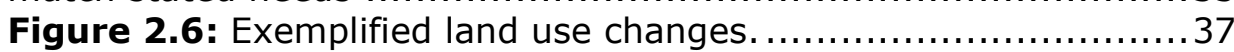

Figure 3.1: Conceptual framework of chapter $3 \ldots \ldots \ldots \ldots \ldots \ldots \ldots . \ldots . \ldots . \ldots . \ldots$

Figure 3.2: Global overview of the current status of UAV regulations

on a country-level resolution.

Figure 3.3: Global distribution of first releases of UAV regulations on country level resolution ................................................. 54

Figure 3.4: Categorisation of UAV operations according to Commission Implementing Regulation 2019/947 ......................56

Figure 3.5: Schematic distinction between UAV flight ranges ........60 Figure 3.6: Consolidated overview of past, present and future developments........................................................... 71

Figure 4.1: Conceptual framework of chapter $4 \ldots \ldots \ldots \ldots \ldots \ldots \ldots . \ldots . \ldots \ldots$

Figure 4.2: Overview of all UAV datasets presented as orthomosaics, scales vary. ............................................................... 79

Figure 4.3: Distribution of GCPs for experimental assessment of the spatial accuracy ......................................................... 83

Figure 4.4: Workflow to define reference lines and a search mask for lines representing concrete walls and rooftops.......................... 85

Figure 4.5: Workflow to compute and select MCG lines representing rooftops or walls and analytical tools to describe geometric characteristics of selected MCG lines. .................................. 86

Figure 4.6: Standardized values of automated tie-points using SIFT, AKAZE, and SURF as feature extraction, detection and matching algorithm............................................................... 87

Figure 4.7: RMSE of checkpoint residuals measured in the DSM (vertical) and orthophoto (horizontal).

Figure 4.8: Selected reference lines representing rooftops (green) and walls (red) for the AOI in Kibonde.

Figure 4.9: Example showing the differences of automatically extracted rooftops and walls 
Figure 4.10: Box-Whisker plot of point distances to reference lines separated according to the reference wall and rooftop....

Figure 4.11: Scatterplot of error metrics for delineated rooftops and walls of orthophotos captured with different flight configurations....96 Figure 4.12: Box-Whisker plot of distances to reference lines separated according to the direction of walls.

Figure 5.1: Nine Pathways of the Framework for Effective Land Administration

Figure 5.2: Prominent innovation curves plotted together........... 110

Figure 5.3: Scheme of the Hype Cycle ................................ 111

Figure 5.4: Scheme of the research approach ...................... 112

Figure 5.5: Scheme of the methodological approach and expected

results.

Figure 5.6: Reflections on the Hype Cycle of Gartner Inc........... 118 Figure 5.7: Combined matrix of FELA and the Hype Cycle with the assessment of the importance in each cell (High- Medium-Low)....120 Figure 6.1: Main conclusions on the dependencies of the nexuses in the socio-technical system including land data needs (chapter 2), UAV regulations (chapter 3 ), data quality (chapter 4 ), and technology diffusion (chapter 5). .................................................. 135

Figure A.1: Concept of the its4land project......................... 160 Figure A.2: Case study area for community-based participatory mapping

Figure A.3: UAV data collection ..................................... 163

Figure A.4: Participatory mapping activity........................ 163

Figure A 5: Data products derived from UAV images................ 164

Figure A.6: Overview of parcel boundaries derived from participatory mapping (red) and cadastral database LAIS (yellow) ..................165 Figure A.7: Percentage of overlay from parcel area derived from participatory mapping with parcel area from LAIS .................... 166

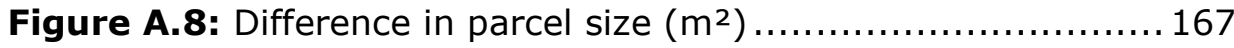

Figure A.9: Statistical analysis of distances of parcel corner points derived from GNSS measurements and participatory mapping (left), graphical examples for a regularly shaped parcel (upper right) and irregularly shaped parcel (lower right) ............................... 168 


\section{List of Tables}

Table 2.1: Types of stakeholders participating in data collection workshops.

Table 2.2: Operational limitations for UAV flight missions in Rwanda

according to Rwandan regulations...................................... 24

Table 2.3: Specifications of UAV used in this study. ...................25

Table 2.4: Block orientation method and ground-truthing information

of all datasets. ......................................................... 27

Table 2.5: Number of deployed reference points - count before and

after the UAV flight

Table 2.6: Land information needs as identified by government

stakeholders. ............................................................... 30

Table 2.7: Land information needs identified by non-government

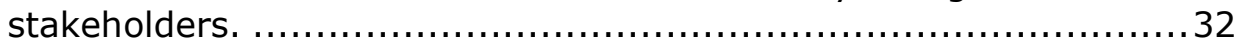

Table 2.8: Specifications of final results.............................. 33

Table 3.1: Overview of online-accessible lists and overviews of UAV

regulations...

Table 3.2: Overview of criteria and variables of the comparative analysis...................................................................... 51

Table 4.1: UAV equipment and specifications of the sensor ..........79

Table 4.2: Specifications of GCP measurements ....................... 80

Table 4.3: Land use classes and representation in datasets (Bentelo,

Gerleve, Kajiado, Kibonde, Muhoza, Mukingo) .......................... 81

Table 4.4: Mean of automated tie-points of image pairs showing

different land use classes and overlap ................................. 88

Table 4.5: Differences of RMSE of checkpoint residuals measured

after the BBA and in the orthophoto/DSM .............................91

Table 4.6.: Qualitative and quantitative characteristics of line geometries representing rooftops (R) and walls (W) separated according to flight configuration and flight pattern ......................93

Table A 1: Comparative analysis of 19 national UAV regulations .. 172 
Chapter 1 - Introduction 


\subsection{Setting the scene}

The World Population Prospects of UN-DESA 2019 states that the global population could grow to around 9.7 billion people in 2050. According to this report, more than half of that global population growth is expected to occur in sub-Saharan Africa (UN-DESA, 2019). This sets its own challenges because all people need shelter and have to be fed in a sustainable way. Amongst others, these basic societal needs require, first of all, land. The livelihoods of many, especially the poorest and vulnerable, are based on access and control over this resource, both in rural and in urban contexts (UN-HABITAT, 2008). In this regard, secure land rights promote tenure security, a critical factor for the eradication of hunger and poverty, the promotion of peace, economic growth, and the sustainable use of the environment (UNFAO, 2012; UN-GGIM, 2015). Accordingly, equal access to basic services, ownership and control over land and other forms of property is one of the targets mentioned in the first goal in the 2030 Agenda for Sustainable Development with the aim to end poverty in all its forms everywhere (UN, 2015). Target 1.4 is assessed by the proportion of the total adult population with secure tenure rights to land, with legally recognized documentation, and who perceive their rights to land as secure, by sex and by type of tenure. However, according to a global analysis of Prindex, nearly 1 in 5 adults feels insecure about their land or property rights (Feyertag et al., 2020), mainly attributed to missing documentation. This global statistic implies that millions of people-toland relationships need to be identified and recorded - the core task of land administration. New tools and approaches are needed to overcome the cadastral divide (Bennett et al., 2013). The its4land project $^{1}$, funded under the Horizon 2020 scheme of the European Commission (project number 687828), investigated several innovative geospatial technologies to accelerate the documentation of land rights. This dissertation explores Unmanned Aerial Vehicles (UAVs) as one of these promising technologies as an innovative tool for responsible land administration.

\subsubsection{Land administration}

By definition, land administration can be understood as the process of recording and disseminating information on ownership, value and use of land and its associated resources (UNECE, 1996). In this context,

\footnotetext{
${ }^{1}$ More information can be found in Appendix 1 and on the its4land homepage www.its4land.com (last accessed 27.07.2021)
} 
the term land is considered with its two dimensions. At first, the physical dimension represents the surface of the earth and all things attached: they are inherently immoveable. In contrast, the abstract dimension of land covers the set of rights residing in the conceptual understandings of people, relating to its use and a specific value (be it economic or spiritual).

A conceptual framework for the holistic view on land administration processes is given by (Enemark, 2004). The operational component of the provided paradigm includes interrelated functions within the area of land tenure, land value, land use and land development - which ensure proper management of rights, restrictions, responsibilities and risks in relation to property, land and natural resources. Seeking for sustainable development, these four land administration functions are facilitated by appropriate land information infrastructures. Therefore, land information and geospatial data serve as reliable base data and thus are crucial to the successful implementation of land policies and strategies (Williamson et al., 2010).

\subsubsection{Land information}

According to (Enemark, 2004), land information ideally combines cadastral and topographic datasets in order to link the built environment (including legal and social land rights) with the natural environment (including topographical, environmental, and natural resource information). A critical aspect of land information refers to the legal and spatial interests of people in land. This dataset and the components used to manage it are called cadastres, which provide parcel-based information about rights, restrictions and responsibilities. The legal extent of cadastral information includes rights, restrictions and responsibilities and is commonly documented in a land register. Meanwhile, the spatial description of size, shape, boundary and location of land parcels is depicted or digitized on cadastral maps. Some argue that the cadastre is the operational core or "engine" of land administration systems (Williamson et al., 2010). Depending on the societal and cultural context of a geographical region, cadastres may be designed in many different ways serving the purpose of taxation and security of land rights.

Conventional cadastral maps are often based on visual boundaries which are surveyed in the field and aim for highly accurate delineation and adjudication of formal property rights, typically ownership and long term leasehold. Whilst most developed countries already maintain wellestablished cadastres, most countries in the Global South do not have 
complete cadastral coverage (GLTN, 2012; Zevenbergen et al., 2013). Notwithstanding political motives and power imbalances contributing to the problem, the limitations of western-oriented land administration systems and traditional surveying approaches, and a substantial lack of capacity to undertake those surveying activities have indisputably contributed to this reality. As a consequence, people cannot claim their legal rights to land. Resulting land tenure insecurity may cause land disputes, instability and hinders effective land markets and investments (Besley, 1995).

Therefore, the overall challenge for the global land community is clear: tenure security for all people, in all places, at all times (Lemmen et al., 2015). Rethinking the way people's relation to land is recorded and managed has led to emerging land administration approaches, variously described as fit-for-purpose, pro-poor, inclusive, progressive, responsible, amongst other terminology. Although they seem to lack a shared agreement on definition (Zevenbergen, De Vries and Bennett, 2015), the underlying principles and characteristics coincide. Core requirements include complex, layered tenures through the continuum of land rights, low-cost land registration, which is affordable to the poorest and to the state, gender equality in relation to tenure security, and finally, flexible and local people-oriented land administration tools (Williamson et al., 2010). The implementation and development of these principles need policies, actors and tools.

\subsubsection{Alternative tools to collect land information}

In the past decades, alternatives to costly classical cadastral surveying techniques with theodolites or high-end GNSS emerged. First and foremost, the involvement of local communities in the mapping process gained importance, as shown during the nationwide project in Rwanda (Ngoga, 2018) or the numerous projects run by international organisations such as Cadasta.org or Namati. In this aspect, two main methods can be distinguished: direct measurements using GNSS sensors and indirect measurements based on maps.

In contrast to high-end surveying equipment commonly used in western-oriented countries, alternative approaches to direct measurements utilize mobile phones or hand-held GNSS devices to determine parcel boundaries by walking the boundary and tracing corner points or lines. As opposed to this, the use of maps for indirect measurements does not require field measurements. Here, satellite images or aerial photography are used as base maps for participatory mapping activities based on the principle to identify physical features 
such as roads, houses, walls or other objects typically representing cadastral boundaries. Various studies prove that such visual boundaries can be identified on plotted satellite images and either be demarcated by local stakeholders in a participatory manner (Asiama, Bennett and Zevenbergen, 2017; Aditya et al., 2020; Panday et al., 2021) or delineated using manual or automatic feature extraction approaches (Crommelinck et al., 2018; Kohli, Unger and Lemmen, 2018). Depending on the topography and land use, different spatial resolutions may be needed (Enemark et al., 2014).

However, satellite images also impose certain challenges, referring to an insufficient spatial resolution which did not allow to identify parcel boundaries in urban contexts (Panday et al., 2021), long and expensive processing times, and challenges to obtain up-to-date data due to frequent cloud cover mainly in tropical regions (Tatham, 2009; Ngoga, 2018). Missions to capture classical aerial photography involve substantial organisational efforts and are less cost-effective for small to medium-sized areas (Matese et al., 2015). In this context, UAVs can mitigate some of these issues and are seen as an alternative tool to capture aerial images for further boundary delineation.

\subsubsection{Unmanned Aerial Vehicles}

Unmanned aerial vehicles (UAVs) - also known as the gender-neutral term unoccupied aerial vehicle (UAV), remotely piloted aircraft systems (RPAS) or just 'drones' - are remotely controlled and follow semiautonomously or autonomously predefined flightpaths. The term unmanned aerial systems (UAS) can also be found throughout the literature and considers the whole system, which includes the unmanned aircraft and the on-ground command-and-control station (Everaerts, 2009). Within the last decade, UAVs became a genuine gain for scientific as well as commercial applications. Since the price and the size of UAVs significantly dropped over the past years (Barnes and Volkmann, 2015), they stand out as an affordable acquisition tool for mapping and investigations at short time frames.

In general, UAV flight missions include both technical and non-technical aspects. As shown in Figure 1.1, the UAV itself, the UAV pilots, and the legal permission to conduct UAV flights are the main requirements for UAV-based mapping. The UAV data acquisition mission follows three phases: flight planning, data acquisition, and data processing. In most cases, most emphasis is put on the UAV flight as the main event. However, it makes up only $20 \%$ of the time effort needed for the entire UAV-based field campaign (Nex and Remondino, 2014). Depending on 
the flight configuration and mission, ground truthing and image processing may be 2-3 times more time demanding than the actual UAV flight.

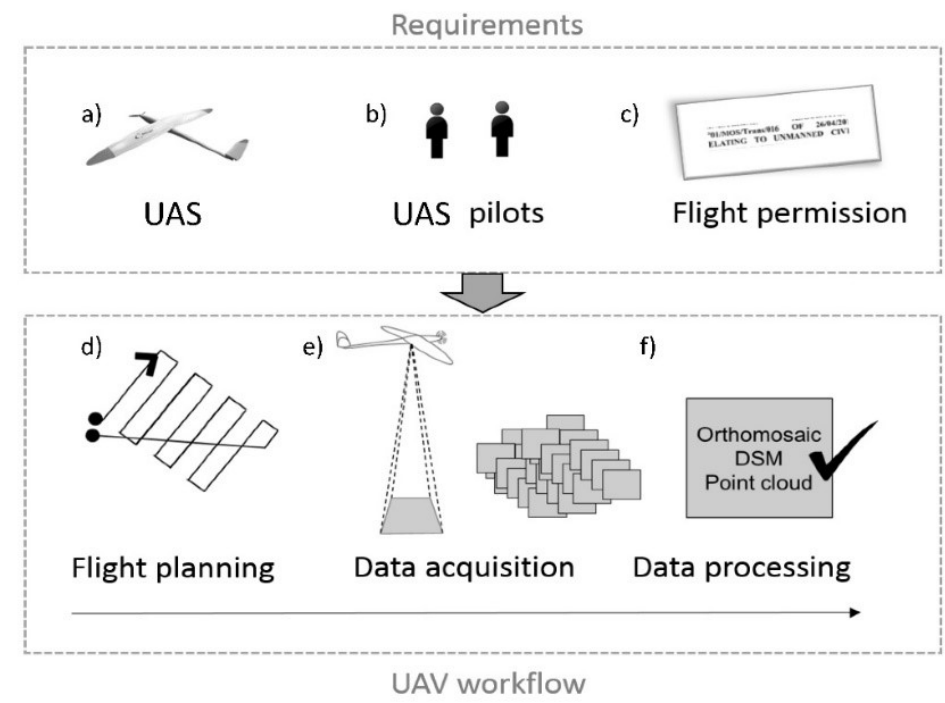

Figure 1.1:UAV-based mapping - requirements and data acquisition workflow. (a) UAV equipment, payload and the ground control station; (b) trained staff to pilot and operate the UAV; (c) legal permission to conduct the UAV flight mission, which can set its own requirements according to the national jurisdiction; (d) flight planning with appropriate software and definition of flight characteristics; (e) data acquisition flight; (f) data cleansing and photogrammetric processing including quality assessment.

Data processing usually follows structure from motion and modern photogrammetric algorithms, which typically require very few operator decisions allowing for a high degree of automation (Colomina and Molina, 2014; Sanz-Ablanedo et al., 2018). Data outputs may include orthoimages, digital elevation models and 3D point clouds which can all serve as possible base data to derive land information. The spatial accuracy of these data outputs depends on many configurations, including sensor specifications, UAV itself, mode of georeferencing, flight pattern, flight height, photogrammetric processing, image overlap, and external variables such as weather, illumination, or terrain.

Subject areas are manifold and include computer sciences and engineering as well as social sciences, earth and planetary sciences. Amongst others, applications comprise high-resolution surface reconstruction in geosciences (Eltner et al., 2015), documentation of 
cultural heritages and archaeological sites (Remondino et al., 2011), agriculture and forest change detection (Grenzdörffer, Engel and Teichert, 2008; Honkavaara et al., 2012; Zhang and Kovacs, 2012) support of disaster management (Tatham, 2009; Adams and Friedland, 2011; Maza et al., 2011), surveying (Tampubolon and Reinhardt, 2014) or cadastral mapping (Mesas-Carrascosa et al., 2014; Barnes and Volkmann, 2015; Mumbone et al., 2015; Koeva et al., 2016).

\subsubsection{UAVs in land administration}

A large body of literature proves that UAVs can bridge the gap between field surveys and space- or airborne surveys and promise flexible, cheap, real-time and fast data acquisition. Characteristics, which are considered key for addressing current land administration challenges.

So far, several studies have investigated the benefits of using UAVs within diverse cadastral applications and highlight versatility and efficiency (Manyoky et al., 2012), the multi-purpose-characteristic (Lee, Kang and Lee, 2016) and the empowerment of local citizens and support of participatory mapping (Hardiono et al., 2016; Meha et al., 2016). (Kelm et al., 2014) and (Ramadhani, Bennett and Nex, 2018) further prove that UAVs can meet the requirements of fit for purpose mapping (Enemark et al., 2014). Furthermore, some authors highlight the easy-to-use and straightforward approach of UAV operations, allowing various stakeholders like individuals, communities or businesses to acquire mapping capacities (Barnes and Volkmann, 2015).

Reported limits of UAV based cadastral mapping refer to harsh environmental conditions such as heat, rain, wind (Rijsdijk et al., 2013; Spectairgroup, 2016), data quality inconsistencies due to insufficient image overlap (Maurice et al., 2015), or motion blur and low-cost camera applications (Manyoky et al., 2012). Furthermore, cumbersome regulatory frameworks and undeveloped ground-truthing strategies were also described as the main issues currently impeding large-scale implementation.

\subsection{Research problem}

UAVs are seen as a widely established technology to gather highresolution imagery for land use or land cover mapping (Colomina and Molina, 2014). Although reported benefits seem to be auspicious, the adoption of UAVs as an innovative tool for cadastral mapping workflows remains remarkably poor. Previous works - which seldom exceed the scope of feasibility studies - mainly refer to data-driven and 
technological assets of the utilization of UAVs and do not consider societal, governance and institutional settings and needs of people and groups. To address this gap, this thesis aligns technology-push with societal-pull by utilizing a socio-technical approach emphasizing technical and social factors. A systems approach addresses the complexity of existing problems by stressing interrelationships and connections between system elements (Montuori, 2011). Both approaches are not new to research in land administration. A sociotechnical approach has already been exemplified for cadastral systems (Ottens and Stubkjær, 2007) and the real estate market (Liddell, 2004). Moreover, (Zevenbergen, 2004) applied a systems approach to land registration and cadastre. Overall, existing works strongly advocate taking a holistic viewpoint instead of single-lens perspectives.

The conceptual framework of this dissertations is based on the interrelationship of three key elements integrated into a holistic framework: technology, processes and actors. These three elements are rooted in the approach proposed by (Leavitt, 1965) which evolved during the past decades to a widely applied framework for organizational change.

As illustrated in Figure 1.2, processes involve land administration processes, technology comprises UAV technology, and actors embrace stakeholders.

Land administration processes include the process of providing spatial information and base data to gather information about rights, restrictions and responsibilities of people to land relationships.

UAV technology includes the hard- and software required to acquire land information data

Stakeholders are seen as people or groups that are directly involved in or affected by this research. Here, public sector entities, nonstatutory entities, private sector entities and NGOs/non-profitorganisations are included.

Above that overarching problem statement, each chapter addresses another underlying problem reflecting on the different viewpoints to investigate a specific interrelationship: 1) land data needs (actors processes), 2) UAV regulations (actors - technology), 3) data quality (processes - technology), 4) technology diffusion (technology-actorsprocesses). 


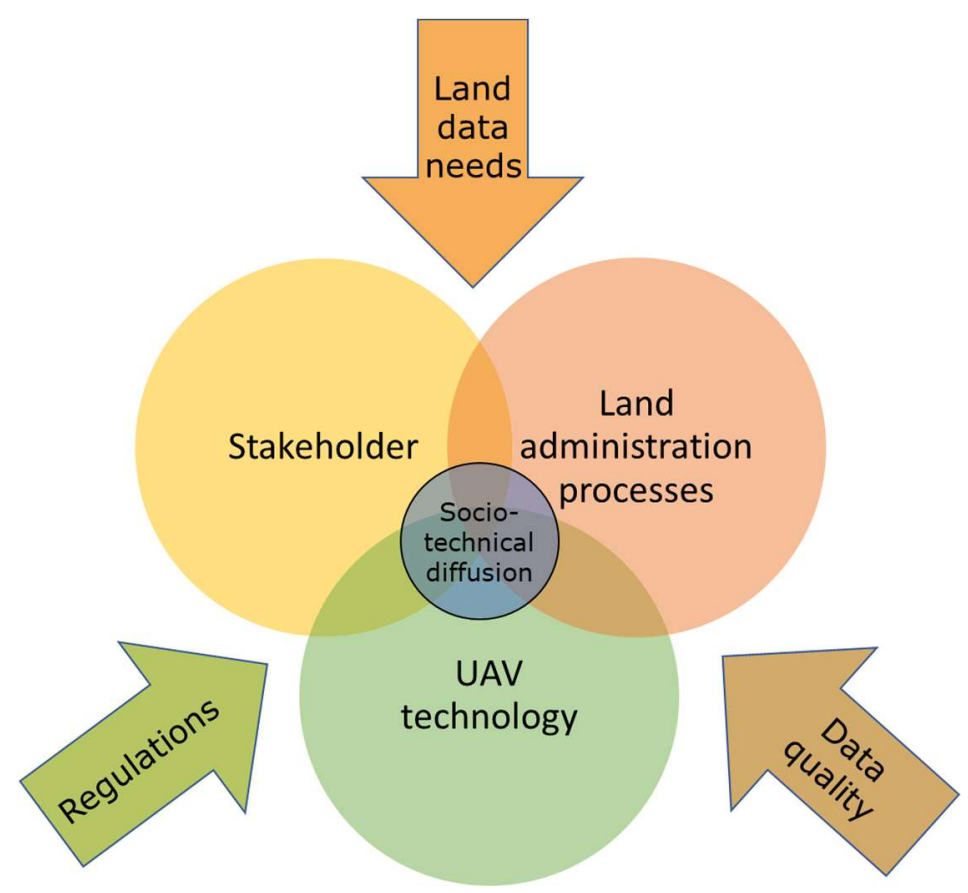

Figure 1.2: Conceptual framework and interrelationship of system elements

The following paragraphs provide a snapshot of the underlying research problems.

\section{Deficient knowledge on how UAV technology can meet land data needs}

A flexible and pragmatic approach to meeting people's needs and their relationship to land refers to the key principle of recent land administration approaches (Zevenbergen et al., 2013; Enemark et al., 2014; de Vries, Bennett and Zevenbergen, 2015). While UAVs appear to be a promising technology, there has been little discussion in the literature as to what extent this technology can match the needs of communities and governments. Unlike leveraging technical standards, these approaches advocate that the data acquisition method of the underlying spatial framework should have a strong focus on managing current land issues in a specific context. Little has been done to study how UAV technologies precisely fit different needs articulated by governmental and non-governmental actors. Hence, a critical examination of stakeholders' needs and how UAV technology can meet those requirements is needed. 


\section{Lack of a global review of UAV regulations}

Recent technological improvements and increasing operational capabilities of UAVs present specific challenges to flight operators, endusers and aviation authorities: especially concerns about privacy, data protection, and public safety (Finn and Wright, 2014). To minimize the risks of UAV-triggered incidents or accidents, an increasing number of national and international authorities have introduced legal provisions that mandate "Go," "No go", or "How to go" statements that either allow, prohibit or restrict flight operations. Such regulations significantly impact how, where, and when data can be captured-and the diffusion of the technology within a national context. As the legal frameworks to operate UAVs are deemed the main driver for technology uptake in almost any application area, it is essential to understand the importance, impact, and diversity of UAV regulations on a global scale.

\section{Absence of guidance for optimal UAV flight configurations to derive high-quality orthophotos}

When UAV-based orthophotos are used to derive spatial information on land rights, incorrect geometries of the base data may cause negative consequences to civil society as the subject deals with the spatial representation of land parcels and attached rights and responsibilities. For example, erroneous localization and estimations of parcel sizes might imply inadequate tax charges, problems with land compensation funds, or challenges to merge existing databases spatially. During the entire UAV workflow, the operator needs to take conscious decisions on various parameters to ensure that expected data quality requirements can be met - a criterion often specified by the concept of spatial accuracy. Most existing work elaborates on means of groundtruthing and georeferencing, undeniably crucial aspects of any UAV flight mission. However, the results of those studies are very heterogeneous, and most of them remain narrow in focus, dealing mainly with only one study site situated in non-populated areas. Thus, it is questionable whether recommendations can be transferred to the cadastral context. Additionally, no research investigated the quality of the spatial representation of typical cadastral features yet, an aspect which is particularly important if the data is being used for (semi-) automated feature extraction. Hence, a comprehensive analysis of varying data quality measures is needed to provide a factual basis for clear recommendations to guide UAV-based cadastral mapping. 


\section{Lack of evidence on the innovation process of UAVs in land administration}

Looking at the prospects of UAV technology, from a global perspective, there has been hype and excitement around UAVs as a disruptor for land administration. In a review, (Floreano and Wood, 2015) do not see significant technological or scientific barriers to advocate UAVs in research and commercial applications. It is often argued that technology needs to be mature enough, economically feasible and efficient to create impact. However, this only considers the (technical) innovation, which does not necessarily guarantee a successful diffusion or adoption. Instead, it has been shown that political, socio-cultural and institutional settings within a system are among the main determinants to steer the transformation of existing land administration processes in favour of innovative technologies (Bennett, Pickering and Sargent, 2019). The studies presented thus far provide evidence that a holistic perspective, including technology and social and institutional systems, is fundamental to understanding the drivers and barriers to scale implementation of innovative technologies. However, in the context of UAVs, a comprehensive global analysis on the requirements and processes needed to support leveraging the technology innovation and to foster its wider adoption and diffusion in land administration contexts is absent.

\subsection{Research objectives}

The main objective of this dissertation is to explore UAV-based data acquisition workflows as a tool for responsible land administration. To achieve this main objective, the following sub-objectives are addressed:

1) To examine stakeholders' land information needs and understand how UAV technology can meet respective requirements.

2) To analyse the interface between UAV data acquisition workflows and stakeholders in terms of legal and regulatory frameworks.

3) To assess different data quality measures of UAV-based orthophotos and recommend optimal UAV data acquisition workflows.

4) To understand the dynamics of the diffusion process of UAV technology as a tool for land administration. 
These research objectives are based on the following definitions:

Unmanned Aerial Vehicles (UAVs) are to be understood as uninhabited and reusable motorized aerial vehicles (van Blyenburgh, 1999).

Data acquisition workflows encompass the whole operational UAV procedure, including planning and preparation, fieldwork, data processing and quality assessment.

$A$ tool is a practical way to solve a problem in land administration and management (GLTN, 2012).

Responsible land administration aligns land administration processes, structures and outcomes with ever-changing dynamics of societal demands (Zevenbergen, De Vries and Bennett, 2015; De Vries and Chigbu, 2017).

\subsection{Methodology}

Overall, this dissertation follows a practice-oriented research approach aiming to support a practical problem to be solved or a decision to be taken (cf. Bleijenbergh, Korzilius and Verschuren, 2011). The authors further outline that, unlike a theoretical problem, a practical problem requires intervention to change reality in the desired direction. In this dissertation, the practical problem refers to the millions of undocumented land rights and the people lacking tenure security. This research explores the opportunities of UAV-based data acquisition workflows as an intervention to support responsible land administration to increase the share of people with secure land rights eventually. It is hoped that this dissertation can guide future decision-making processes related to the application and implementation of UAV-based data acquisition workflows to support land administration efforts at a national, regional or local scale.

This thesis lies at the intersection of remote sensing, land administration, and information systems to account for this objective. In general, the choice of a research methodology is primarily driven by the research objective itself and should be according to the research situation (Yin, 2003). Thus, different research methodologies are applied to cater to the contextual variety of sub-questions posed, each targeting another nexus of the system elements actors, processes, and technology. The following paragraphs briefly describe the selected methods. A detailed description of experiments, data collection strategies and study areas is provided in each chapter. 
The first objective seeks to match land data needs with specifications of UAV data collection workflows. A mixed-methods approach is applied to integrate, mix, analyse, or collect both qualitative and quantitative data within one single study. On the one hand, land information needs of various stakeholder groups are identified through a group interview process to capture the group's ideas and provide deep and meaningful results ranked by importance to the topic of interest. On the other hand, experimental test flights provide input data to evaluate the institutional environment and the quality of data outputs. Rwanda serves as a case study location to reach this objective. The mixedmethods approach is vital in this chapter as in neither case, one kind of data would be sufficient, by itself, to capture a holistic understanding of the characteristics of a particular situation (Tashakkori and Teddlie, 1998).

The second objective seeks to analyse the interface between UAV technology and stakeholders in terms of legal and regulatory frameworks. To reach this objective, a research synthesis is carried out using existing facts and multiple sources to generalise the topic of research (Cooper and Hedges, 2009). Data sources are manifold, including regulatory frameworks, scientific articles, and grey literature. The first pillar of the research synthesis involves a comparative analysis of the various documents that regulate UAV operations in 19 countries. In addition, a review of the scientific literature focussing on the relationship between law, innovation, and technology constitutes the second pillar. Together, both pillars of the research synthesis provide a comprehensive overview of UAV regulations and their implications for flight operations and infer on past, present and future developments.

The third objective recommends optimal flight configurations and thus requires assessing the data quality of differently acquired and processed UAV-based orthophotos. An experimental setup includes UAV imagery from six study areas across Europe and Africa and more than 100 scenes representing different flight configurations. The statistical quality analysis incorporates three main aspects: (1) the impact of land cover on the number of tie-points as an indication of how well bundle block adjustment can be performed, (2) the impact of the number of ground control points (GCPs) on the final geometric accuracy, and (3) the impact of different flight plans on the extractability of cadastral features.

The fourth objective seeks to understand the dynamics of the diffusion process of UAV technology as a tool for land administration and draws on observations and experiences of experts in the land 
administration and UAV sector. Data on the progression of UAV technology in land administration stems from a qualitative content analysis of interview transcripts from nine semi-structured expert interviews. The theoretical model developed in this chapter fuses established models from land administration and information systems and proposes a new framework to analyse the emergence of innovative technologies for effective land administration. Given the relatively limited amount of "real" practical projects in this sector, this research is more explorative than confirmative. It proposes a scientific foundation to understand the requirements to scale up the use of UAV technology in land administration.

\subsection{Outline of the thesis}

The overall structure of the thesis takes the form of six chapters, including an introductory chapter, four chapters that elaborate on the research questions, and the last chapter, which summarises the key findings and outlines the implication of the results. The main chapters are derived from scientific articles, with each publication addressing one sub-objective, thus, reflecting on one specific interrelationship as shown in Figure 1.3.

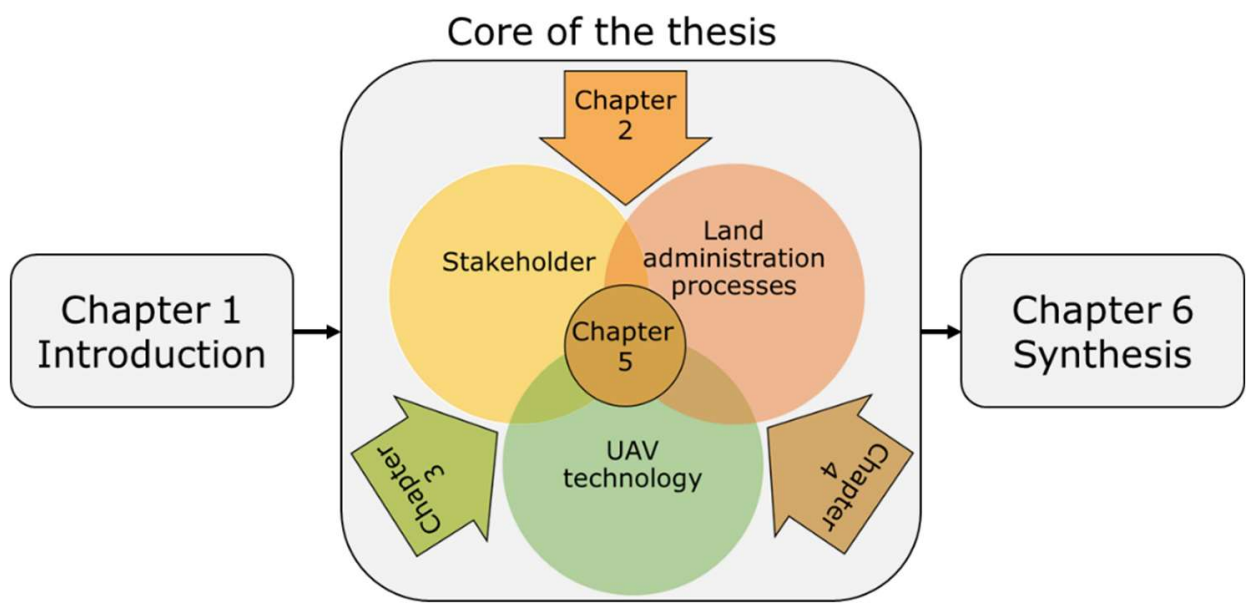

Figure 1.3: Organization of this dissertation

Each of the main chapters includes a brief background section, outlines materials and applied methods, and presents and discusses the results. With this design, chapters may slightly overlap in the introduction and motivation but can be considered individually according to the reader's interest. 
Chapter 1 - introduces this dissertation by sketching the research problem, outlining the research questions, and describing each chapter's research methodology and content.

Chapter 2 - considers the nexus between stakeholders and land administration processes by investigating land data needs. Results of the needs assessment are compared with characteristics of UAV data acquisition workflows and specifications of data outputs. Thus, emphasis is put on technical as well as institutional elements. This analysis is complemented with a practical excursus testing and evaluating a UAV-driven community mapping process (see Appendix 2 ). It results in a matrix highlighting four main characteristics of UAV data and associated land data needs.

Chapter $\mathbf{3}$ - investigates the nexus between stakeholders and UAV technology in terms of legal and regulatory frameworks surrounding the commercial operation of UAV technology. This chapter identifies past, present and future trends and core themes of current UAV regulations, often considered one of the main hurdles to successful UAV operationalization.

Chapter 4 - focuses on the nexus between land administration processes and UAV technology and emphasizes data quality as one of the critical determinants for choosing an appropriate surveying technique. Various data quality measures, as well as different groundtruthing strategies and flight configurations, are analysed. A detailed analysis of the findings allows deducing recommendations for optimal flight configurations for UAV-based data acquisition.

Chapter 5 - analyses the diffusion process of the use of UAV technology in land administration. Models from innovation processes and effective land administration are integrated to derive insights on development stages and key aspects at a given time. This chapter reflects on past and present developments and indicates possible future advancements to reach the plateau of productivity.

Chapter 6 - synthesizes the results of the core chapters and outlines the implications of this research to science, land administration, and UAV practice and policy. 


\section{Chapter 2 - UAV imagery, Land Data and User Needs $^{2}$}

2 This chapter is based on:

Stöcker C, Ho S, Nkerabigwi P, Schmidt C, Koeva M, Bennett R, Zevenbergen J. Unmanned Aerial System Imagery, Land Data and User Needs: A SocioTechnical Assessment in Rwanda. Remote Sensing. 2019; 11(9):1035. https://doi.org/10.3390/rs11091035 


\subsection{Introduction}

Compared to other remote sensing techniques such as satellite images or classical aerial images, UAV data has clear advantages regarding the spatial resolution, which is often below $10 \mathrm{~cm}$ and provides a high level of detail. To reach low ground sampling distances, flight height is usually set to less than $100 \mathrm{~m}$ - a limitation given mainly by the national UAV regulations. Thus, the scale of one UAV mission is small, reaching from a few hectares up to hundreds of hectares, depending on the platform, the field of view of the sensor, image overlap and flight pattern. Thus, aerial/satellite images are more suitable for largescale mapping. With a particular focus on land rights recording, (Stöcker, Koeva, et al., 2019) concluded additional advantages of UAV data collection workflows: reliability of the data, open and transparent data collection procedure and ease of implementation. The latter parameters are of particular importance to the implementation of a fitfor-purpose land administration tool with a strong focus on developing countries.

While UAVs appear to be a promising technology, there has been little discussion in the literature as to what extent this technology can match the needs of communities and governments, especially when land administration is absent, incomplete, or in a state of decay. A flexible and pragmatic approach to meet the needs of people and their relationship to land refers to the key principle of recent land administration approaches (Zevenbergen et al., 2013; Enemark et al., 2014; Zevenbergen, De Vries and Bennett, 2015). Unlike leveraging technical standards, these approaches advocate that the data acquisition method of the underlying spatial framework should have a strong focus on managing current land issues in a specific context. Little has been done to study how different innovative geospatial technologies fit different needs.

Therefore, this chapter aims to critically examine the match between stakeholders' needs and the characteristics of the UAV data acquisition workflow and its final products as valuable spatial information for land administration. This was achieved through undertaking a case study in Rwanda where a mixed-methods approach was applied. First, the needs of potential end-users were investigated; second, the UAV technology was trialled in Rwanda; third, the performance of the entire UAV-based data acquisition workflow and its ability to match end-user requirements was assessed. A combined analysis of qualitative and quantitative results provides the empirical basis for discussing the degree of fitness of UAV technology for matching users' needs. The 
integration of the results makes this chapter a significant contribution as it reveals the opportunities and limitations of UAV technology in the context of current discourses in land administration.

The remainder of this chapter is organized as follows. After a short overview of the study area in Rwanda, the third section describes the research methodology. The fourth section presents the results focusing on the needs assessment, the UAV test flight missions, and a fusion of both data, which ultimately debates the fitness of UAV technology to attain land administration and spatial planning processes. The discussion relates the results of this study to existing scientific investigations and further reflects on the significance of the work. The conclusion with opportunities and remaining challenges as well as a future outlook complete this chapter.

\subsection{Study Area}

With an area of over $26,000 \mathrm{~km} 2$ and a population of almost 12 million people, Rwanda is the most densely populated country in Africa (467 per $\mathrm{km}^{2}$ ) (National Institute of Statistics Rwanda, 2014). The same report further outlines that the population of Rwanda is still primarily rural, with $83 \%$ living in rural areas, with the majority depending on subsistence farming. However, most agricultural workers own less than 0,5 ha of land or none at all (UNDP and Government of Rwanda, 2015). Despite its land scarcity and prevalence of hilly landscapes, the country continues to be highly reliant on agriculture as a form of employment and subsistence, and an increasing population exerts a growing demand for housing and infrastructure. After independence in 1962, land ownership in the country has evolved from customary law to a system of state ownership. This shift was formalized by implementing a new land policy in 2004 and the Organic Land Law (OLL) in 2005, which aimed to improve tenure security through land registration, facilitate the development of an equitable land market in Rwanda and promote the sustainable use of land. In approximately 2013, a countrywide land tenure regularization program (LTRP) was completed. More than 11 million parcels were demarcated, and almost 9 million parcels were titled to offer Rwandan citizens a range of perceived social, legal, and economic benefits. The LTRP used $96 \%$ aerial images captured in 2008 and 2009 and 4\% satellite imagery as base data to demarcate and adjudicate parcel boundaries in a community-mapping exercise (Gillingham and Buckle, 2014). Geo-information derived from the LTRP has also enabled the development of a national cadastral map (titlebased land administration system), which now underpins a range of 
purposes (Ngoga, 2018). However, base data has not been updated since and geo-information is still based on orthoimages from 2008/2009.

When it comes to the organized use of UAV, Rwanda can be considered progressive compared to other East African countries. At the 2017 World Economic Forum in Davos, high-level delegates from the Government of Rwanda promoted Rwanda as the first country to adopt performance-based UAV regulations. They further outline that the development of infrastructure and policy frameworks will spur business growth and social impact. In October of 2016, Zipline and the Government of Rwanda launched the world's first national drone delivery service to make on-demand emergency blood deliveries to transfusion clinics across the country. After initial difficulties to receive permission to operate beyond the visual line of sight, the business experienced constant growth. In addition to introducing new products, Zipline plans to build a second distribution centre in the east of Rwanda (Rosen, 2017). Besides foreign businesses, local UAV companies such as Charis UAS Ltd. provide professional services in various UAV industries, including mapping, crop monitoring, surveying and aerial photography.

\subsection{Material and Methods}

This chapter employs a mixed-methods approach, including qualitative and quantitative data, to assess the potential of UAV technology to meet land administration requirements in developing countries. The research framework addresses both the social/institutional and the spatial/technical perspective (Figure 2.1). On the one hand, land information needs of various stakeholder groups are identified through a needs assessment process. On the other hand, case studies of multiple test flights provide input to evaluate the institutional environment and data quality of UAV-based orthoimages. Results are synthesized and jointly discussed to better understand UAV technology as a fit-for-purpose tool in the context of land administration (Enemark et al., 2014) and how policies can build on this. 


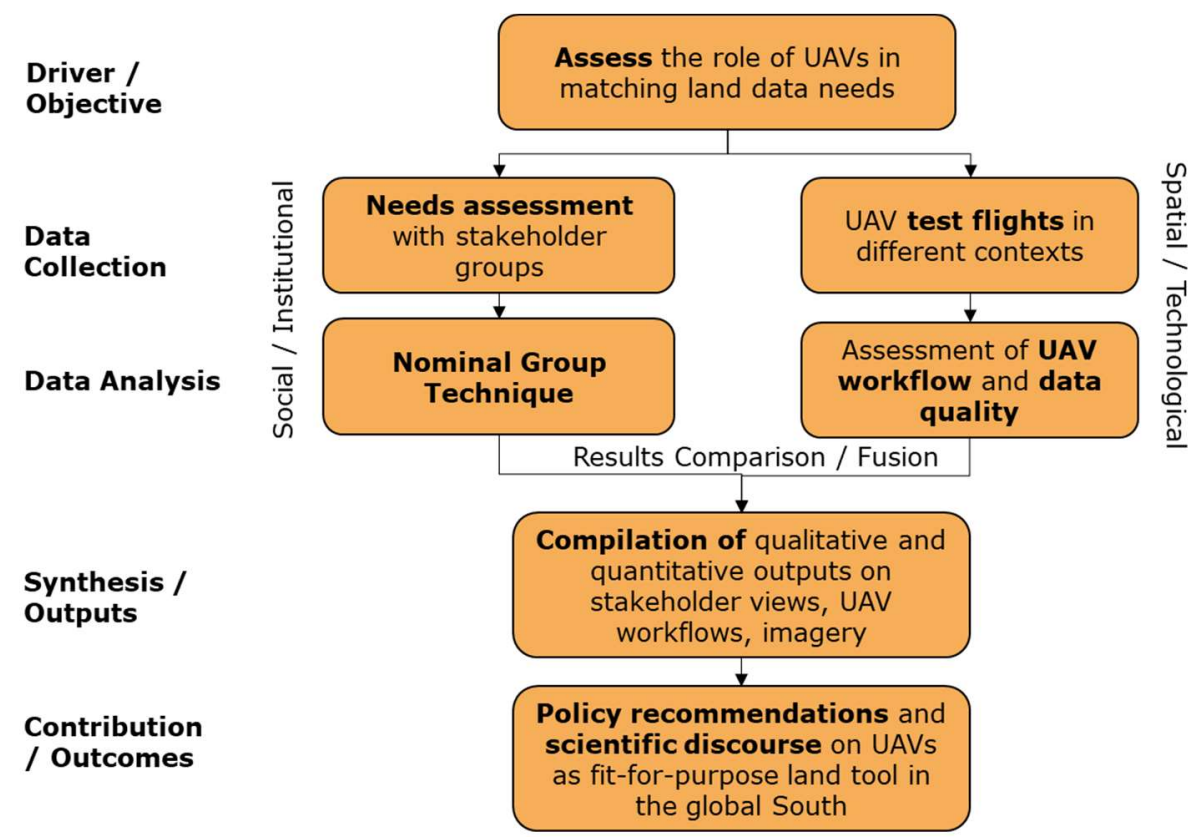

Figure 2.1: Conceptual framework of chapter 2.

\subsubsection{Needs Assessment}

Land information needs assessment for Rwanda was conducted using a form of group interview known as the Nominal Group Technique (NGT). NGT was selected as it facilitates a balanced input from all participants, taking advantage of individuals' knowledge and experience to provide deep and meaningful results ranked by importance to the topic of interest (Delbecq, Van de Ven and Gustafson, 1975). NGT is an effective approach when an identified problem requires a group's ideas and evaluation and is therefore wellsuited for conducting a needs assessment (Sink, 1983; Witkin and Altschuld, 1995; WBI Evaluation Group, 2007). Only one to two questions are posed to the group during the session, as each question takes around two hours to complete. A response to the question in terms of ideas are generated individually then gathered and combined as a group. Group consensus is reached through two rounds of individual voting, a process that prioritizes ideas and provides insight into the extent that individual participants agree or disagree with the consensus vote. This structured process has been proven to effectively address power imbalances or dominant behaviour in group data collection, like some participants being more vocal than others (Delbecq and Van de Ven, 1971; Langford, Schoenfeld and Izzo, 2002; Lloyd, 2011). 
Validity in the method is accounted for by recruiting participants who are considered experts on the topic (Cantrill, Sibbald and Buetow, 1996). Hence, participants were identified by local land administration experts using purposive and snowball sampling. Thirty-eight organizations were contacted; of these, 22 participated (58\% response rate). Three workshops were held at local and national levels. Invited organisations included national and local (district, sector and cell levels), public sector organisations associated with land (e.g. planning, housing, registration, infrastructure, development), non-statutory organisations, private sector organisations (e.g. leading geospatial consultancies), and several universities (Table 2.1). Invitations were sent to senior executives within organizations, and it was left to the organization to send the most appropriate representative to the workshops. For national workshops, attending participants tended to be middle- or senior-level managers; at the local level, attending participants tended to be frontline operational staff.

At each workshop, one nominal question was posed (due to time limitations): "What land tenure and land-related information are still needed for sustainable urbanization?". This was followed by a discussion on how UAV might meet these needs. Cell (smallest administrative entity in Rwanda) officials who could not attend the workshops were interviewed individually using an adapted version of the NGT. Data collection ceased after six interviews when no significantly different insights were gained after four interviews.

Table 2.1: Types of stakeholders participating in data collection workshops.

\begin{tabular}{lll}
\hline \multirow{2}{*}{ Stakeholder Class } & \multicolumn{2}{l}{ Organisations } \\
\cline { 2 - 3 } & Contacted & Participated \\
\hline Public sector organizations specific to & & 12 \\
$\begin{array}{l}\text { land administration (national, province, } \\
\text { district, sector, cell levels) }\end{array}$ & 12 & \\
$\begin{array}{l}\text { Public sector organizations (adjacent } \\
\text { domains to land) }\end{array}$ & 12 & 3 \\
Non-statutory organizations & 1 & 1 \\
Private sector organizations & 3 & 3 \\
NGOs, Not-for-profit/Donors and & 6 & 0 \\
Development partners; & 4 & 3 \\
Research \& Development (R\&D) & & \\
\hline
\end{tabular}




\subsubsection{UAV Data Collection}

The UAV data collection aimed to provide an accurate orthophoto of the study area as base data to derive further land information. Flight planning, data acquisition, and data processing were executed accordingly. However, before the data acquisition commenced attention needed to be drawn on UAV regulations.

\subsubsection{UAV Regulations in Rwanda}

UAV related regulations are a vital requirement in the safe and successful use of UAV technology. In May 2016, the Ministerial Regulations $\mathrm{N}^{\circ} 01 / \mathrm{MOS} / \mathrm{Trans} / 016$ relating to UAV use in Rwanda were officially gazetted (Rwanda Civil Aviation Authority, 2016). Respective regulations are very prescriptive and contain subparts dealing with UAV registration and marking, privacy and safety, airworthiness certification, operating rules and pilot licensing (Stöcker, Bennett, et al., 2017). Before any commencement of activities, the UAV needs to be registered and marked with a unique identifier. Furthermore, pilots and operating agencies need to hold specific licenses issued by Rwanda Civil Aviation Authority. These requirements demand a high standard of UAV professionality and make it a challenge for external companies and institutions to obtain legal flight permissions. At the time of writing, the authors were yet to complete the administrative procedure required (despite commencing the process in 2017) to operate a UAV in Rwanda. Therefore, Charis UAV Ltd., a Rwandan company specialising in UAV services, carried out all data collection flights. The authors' experiences with the UAV regulations and respective governmental institutions point to very high institutional barriers for market entry. Only one company is a certified UAV operator (for landrelated mapping) and arguably has a monopoly. For the specific case related to the work at hand, processes were not transparent and slow with limited access and availability of authoritative, unambiguous and assured information. Although UAV regulations are in place, gaps and lack of capacity can be seen in enforcement and implementation.

Besides requirements for pilot certification, UAV registration, and operator certification, Rwandan UAV regulations outline several operational limitations that must be considered during all UAV flight missions (Table 2.2). Most specifications reflect common restrictions (Stöcker et al., 2018) except for the lateral distance between the pilot and the UAV. Even though the visual line of sight remains undefined, the maximum lateral distance of the pilot to the UAV in operation was set to $300 \mathrm{~m}$ in 2016. This imposed a substantial constraint on UAV 
mapping projects. However, in 2018, UAV regulations were revised, and the maximum lateral distance disappeared from the restrictions, and the flight height was lifted to $120 \mathrm{~m}$ (Rwanda Civil Aviation Authority, 2018). Specifications of restricted areas and requirements towards distances to structures and people are comparable to standard practice.

Table 2.2: Operational limitations for UAV flight missions in Rwanda according to Rwandan regulations (Rwanda Civil Aviation Authority, 2016, 2018).

\begin{tabular}{ll}
\hline Operational Limitation & Specification \\
\hline $\begin{array}{l}\text { Maximum take-off weight } \\
\text { Time for UAV operation }\end{array}$ & $25 \mathrm{~kg}$ \\
$\begin{array}{l}\text { Minimum distance to an } \\
\text { aerodrome }\end{array}$ & Only daylight operation \\
$\begin{array}{l}\text { Maximum flight height } \\
\text { Visual Line Of Sight }\end{array}$ & $10 \mathrm{~km}$ \\
$\begin{array}{l}\text { Maximum lateral distance pilot to } \\
\text { UAV }\end{array}$ & $\begin{array}{l}\text { Required but undefined } \\
\text { Minimum lateral distance to }\end{array}$ \\
$\begin{array}{l}\text { people, vessels, animals, buildings } \\
\text { and structures }\end{array}$ & $30 \mathrm{~m}$ (increased to $100 \mathrm{~m}$ in 2018) \\
Restricted areas & Congested areas of cities, towns or \\
& $\begin{array}{l}\text { settlements } \\
\text { Respect privacy of others, surveillance of } \\
\text { Ethics and privacy }\end{array}$ \\
& $\begin{array}{l}\text { people and property without their } \\
\text { consent is prohibited }\end{array}$ \\
\hline
\end{tabular}

\subsubsection{UAV Equipment}

Three different types of UAVs were tested in this study to assess the variety of UAVs as a platform: one rotary-wing UAV (Inspire 2), one hybrid UAV (FireFLY 6) and one fixed-wing UAV (DT18). The consciously chosen platforms have different specifications in terms of operability, coverage, price, and necessity of ground control measurements. This study set-up reflected the broad spectrum of commonly used UAVs and allowed to acknowledge these varieties within assessing the fitness of use. All platforms were equipped with an RGB sensor to capture nadir images (Table 2.3). The Inspire2 from DJI refers to a semi-professional UAV with a focus on filmmaking. Both the FireFLY6 and the DT18 PPK are survey-grade UAVs, of which the FireFLY 6 presents a lower-cost solution, and the DT18 PPK refers to a professional UAV with high-end components. The DT18 PPK is equipped with a combined Inertial Measurement Unit/ GNSS solution from 
Applanix (APX15), allowing direct georeferencing and minimising the need for ground control measurements.

Table 2.3: Specifications of UAV used in this study.

\begin{tabular}{|c|c|c|c|}
\hline Name & $\begin{array}{l}\text { Inspire } 2 \\
D J I\end{array}$ & $\begin{array}{l}\text { FireFLY6 } \\
B I R D S E Y E V I E W\end{array}$ & $\begin{array}{l}\text { DT18 PPK } \\
\text { Delair Tech }\end{array}$ \\
\hline Type & Rotary wing UAV & Hybrid UAV & Fixed-wing UAV \\
\hline Sensor & Zenmuse X5S & SONY A6000 & DT18 3Bands PPK \\
\hline Sensor size & $13 \times 17.3 \mathrm{~mm}$ & $23.5 \times 15.6 \mathrm{~mm}$ & $8.45 \times 7.07 \mathrm{~mm}$ \\
\hline Pixel pitch & $2.48 \mu \mathrm{m}$ & $3.92 \mu \mathrm{m}$ & $3.45 \mu \mathrm{m}$ \\
\hline $\begin{array}{l}\text { Sensor } \\
\text { resolution }\end{array}$ & $\begin{array}{l}5280 \times 3956 \\
(20.1 \mathrm{MP})\end{array}$ & $\begin{array}{l}6000 \times 4000 \\
(24 \mathrm{MP})\end{array}$ & $\begin{array}{l}2448 \times 2048 \\
(5 \mathrm{MP})\end{array}$ \\
\hline Area & $\begin{array}{l}\text { Busogo (50 ha) } \\
3 \text { flights }\end{array}$ & $\begin{array}{l}\text { Muhoza ( } 94 \text { ha) } \\
2 \text { flights }\end{array}$ & $\begin{array}{l}\text { Gahanga (14 ha) } \\
1 \text { flight }\end{array}$ \\
\hline $\begin{array}{l}\text { Data } \\
\text { collection }\end{array}$ & $\begin{array}{l}497 \text { nadir images } \\
\text { (total flight time: } \\
45 \mathrm{~min} \text { ) }\end{array}$ & $\begin{array}{l}991 \text { nadir images } \\
\text { (total flight time: } \\
60 \mathrm{~min} \text { ) }\end{array}$ & $\begin{array}{l}372 \text { nadir images } \\
\text { (total flight time: } \\
20 \mathrm{~min} \text { ) }\end{array}$ \\
\hline $\begin{array}{l}\text { Main } \\
\text { features }\end{array}$ & $\begin{array}{l}\text { Versatility, } \\
\text { Requires only } \\
\text { small space for } \\
\text { landing }\end{array}$ & $\begin{array}{l}\text { Flight stability, } \\
\text { Requires only small } \\
\text { space for landing, } \\
\text { Long endurance }\end{array}$ & $\begin{array}{l}\text { Long flight } \\
\text { endurance, } \\
\text { PPK-capable, } \\
\text { Automatic flight } \\
\text { and landing mode }\end{array}$ \\
\hline
\end{tabular}

During flight planning, the first step for the UAV data collection, areas for take-off and landing, the UAV trajectory and the flying height are specified. A typical procedure to create the flight trajectory with strips is $80 \%$ forward overlap and $40-80 \%$ side overlap (Colomina and Molina, 2014) since redundancy can compensate for aircraft instabilities. This study's flight planning configurations were constrained by the regulations (operational limitations), requirements for accurate data in an urban environment, and external flight conditions such as wind and illumination. Therefore, considering all these factors, the flight missions were carried out with $80 \%$ forward and $70 \%$ side overlap. According to the regulations, flight height was set to $100 \mathrm{~m}$ above the surface. All UAVs were equipped with an RGB sensor, and resulting ground sampling distances varied between 2$3 \mathrm{~cm}$ depending on the camera's specification. 
Data collection included three different contexts to emphasize the diversity of possible flight configurations - one urban study area, one peri-urban, and one rural study area. The location of the study sites is visualized in Figure 2.2.

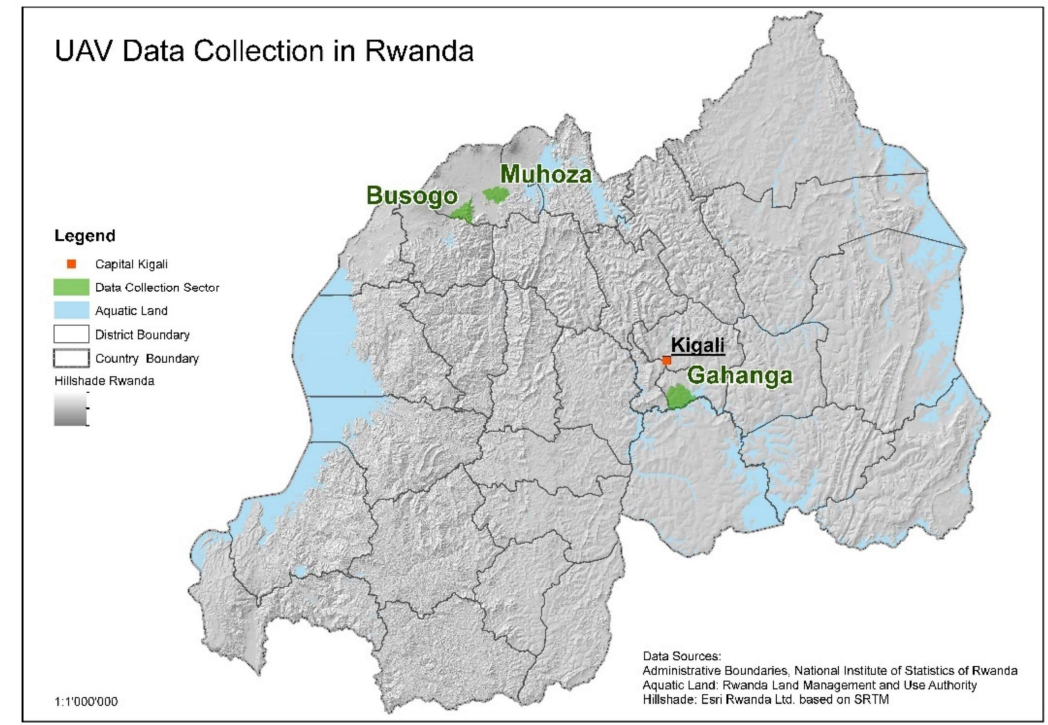

Figure 2.2: Overview of UAV data collection sites in Rwanda.

Due to the limited availability of open spaces, a hybrid UAV for the data collection of the densely populated urban environment in the Muhoza Sector was chosen. In contrast, the rotary-wing UAV was used to collect the images of the peri-urban area in the Busogo Sector. It is located on volcano slopes and is highly affected by local wind systems, which strongly influences the stability of fixed-wing UAVs. Due to the regulatory restrictions and the difficulty of finding good large landing sites, the fixed-wing flight was conducted from a cricket stadium 20 $\mathrm{km}$ south of Kigali. At the time of data acquisition, the maximum lateral distance of the pilot to the UAV was limited to $300 \mathrm{~m}$. Since only one area provided sufficient space for landing, the DT18 could only capture images over the cricket stadium embedded in a rural area in Gahanga Sector. Both the Inspire 2 and the FireFLY6 were equipped with a consumer-grade GNSS antenna allowing geotagging of all images. However, the measurement of additional Ground Control Points (GCPs) is indispensable. In contrast, the DT18 stood out for its high-quality navigation sensor that records detailed attitude logs, including both angular observations ( $<10$ arcmins) as well as camera positions $(<2.3$ cm) (Stöcker, Nex, et al., 2017). However, former test flights showed 
that the DT18 requires additional GCPs to correct for (minor) systematic errors, particularly when no GNSS corrections are applied.

\subsubsection{Ground Control Measurements}

Reference points were deployed and measured in all three case locations to include known point coordinates for georeferencing and a means for quality control. The visible ground marker had a quadratic shape with an edge length of $30 \mathrm{~cm}$ showing a black and white chess pattern (cf. Figure 2.3) evenly distributed in the area of interest. As specified in Table 2.4, ground truth measurements were carried out with two different GNSS devices. The first was a pair of Leica CS10 stations used in a base and rover set with an absolute RTK measurement accuracy of $2 \mathrm{~cm}$. The second device used was a handheld Trimble GeoXH receiving RTK corrections via the Rwandan Continuously Operating Reference Station (CORS) GeoNet with an absolute measurement accuracy of $10 \mathrm{~cm}$. Whereas GCPs were included as a weighted observation during the photogrammetric image processing (Remondino et al., 2011), checkpoints were not considered during image processing and present as a classical way to evaluate the geometric accuracy.

The georeferenced orthomosaic has been generated following two different block orientation methods. The Gahanga dataset was processed employing an integrated sensor orientation method (Benassi et al., 2017) that uses the information of camera positions and attitude and object coordinates of GCPs for the Bundle Block Adjustment. Since no attitude measurements were available for the FireFLY 6 and the Inspire 2, the block orientation of the Muhoza and Busogo dataset followed the GNSS-supported Aerial Triangulation method based on information on camera positions and object coordinates of GCPs (cf. Benassi et al., 2017).

Table 2.4: Block orientation method and ground-truthing information of all datasets.

\begin{tabular}{|c|c|c|c|c|}
\hline Dataset & Block Orientation Method & GNSS Device & $\begin{array}{l}\text { Count } \\
\text { GCPs }\end{array}$ & $\begin{array}{l}\text { Count } \\
\text { CPs }\end{array}$ \\
\hline Muhoza & $\begin{array}{l}\text { GNSS-supported Aerial } \\
\text { Triangulation (GNSS-AT) }\end{array}$ & $\begin{array}{l}\text { Leica CS10 + } \\
\text { Trimble GeoXH }\end{array}$ & 9 & 20 \\
\hline Busogo & $\begin{array}{l}\text { GNSS-supported Aerial } \\
\text { Triangulation (GNSS-AT) }\end{array}$ & Leica CS10 & 9 & 9 \\
\hline Gahanga & $\begin{array}{l}\text { Integrated Sensor } \\
\text { Orientation }\end{array}$ & Trimble GeoXH & 5 & 8 \\
\hline
\end{tabular}




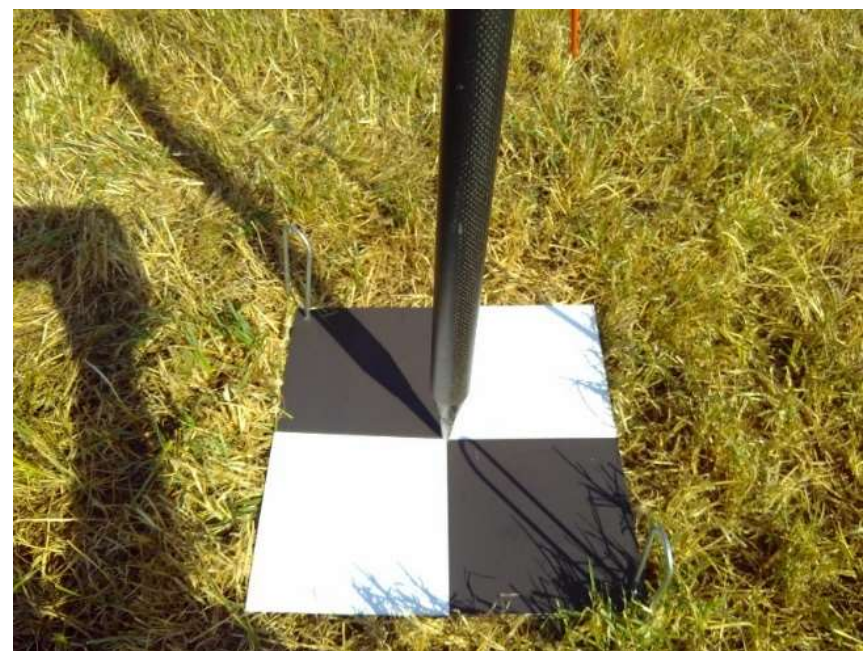

Figure 2.3: Measurement of reference points.

Although less than ten reference points are sufficient to achieve high geometric accuracy, redundancy in deployed points has proven to be the preferable option as ground markers might vanish or be destroyed. Due to unforeseen administrative problems, the time between the deployment of the ground marker and the UAV flight itself was almost $5 \mathrm{~h}$. This can explain that nearly $25 \%$ of all deployed points in the area of Muhoza were taken away.

As summarised in Table 2.5, the authors experienced less time delay and fewer losses of the ground marker in the peri-urban and the rural regions of Busogo and Gahanga.

Table 2.5: Number of deployed reference points - count before and after the UAV flight

\begin{tabular}{lllll}
\hline Area & $\begin{array}{l}\text { Teams } \\
\text { De- } \\
\text { ployed }\end{array}$ & $\begin{array}{l}\text { Reference } \\
\text { Points } \\
\text { Measured } \\
\text { Pre-Flight }\end{array}$ & $\begin{array}{l}\text { Reference } \\
\text { Points } \\
\text { Remained } \\
\text { Post-Flight }\end{array}$ & $\begin{array}{l}\text { Time between } \\
\text { Measurement } \\
\text { and Final } \\
\text { Collection of } \\
\text { Ground Marker }\end{array}$ \\
\hline Muhoza & 2 & 39 & 30 & $5 \mathrm{~h}$ \\
Busogo & 1 & 22 & 18 & $3 \mathrm{~h}$ \\
Gahanga & 1 & 13 & 13 & $2 \mathrm{~h}$ \\
\hline
\end{tabular}

\subsubsection{Software and Hardware Requirements}

UAV data has been processed with the commercially available software Pix4D, which refers to a well-established professional photogrammetric software. Next to commercially available software, freely available 
software for UAV image processing, such as Open Drone Map, offer viable alternatives. Open Drone Map follows a structure-from-motion pipeline which is based on Open SFM. Whereas previous open-source software most often had the deficiency of missing an intuitive, userfriendly user interface, Open Drone Map can be used as a commandline tool, with a live USB or via a user-friendly Web-based graphical user interface. Recommended system requirements are similar for Pix4D and Open Drone Map, and refer to 16 GB RAM for small projects over with 100-500 images, 32 GB RAM for projects over 500 images and 64 GB RAM for extensive projects with over 2000 images. The photogrammetric processing in this chapter was completed with Pix4D and took several hours for the Gahanga and Busogo dataset and more than a day for the Muhoza dataset.

\subsection{Results}

\subsubsection{What Land Information do Rwandan Stakeholders Need?}

The needs assessment results revealed that land information needs were not merely about data but also other enabling requirements such as access, functionality, and tool types. Table 2.6 and Table 2.7 show the outcomes of workshops with government and non-government stakeholders and how final decisions around land information needs were prioritised. The column 'relative importance' reflects the proportion of votes awarded to a specific need, while 'popularity' reflects the proportion of participants who voted for that need.

\subsubsection{Government Stakeholders' Needs}

Spatial data with high accuracy (although participants did not quantify this) related to land tenure and other land information was a priority for national and sub-national government stakeholders, attracting almost $20 \%$ and $30 \%$ of the total votes, respectively. At national and sub-national levels, the emphasis was less on land tenure information and more on other types of land information such as utilities, existing developments and land use, climatic and topographic data. Additionally, there was an emphasis on capacity needs (usability and accessibility), indicated in needs such as integration of land parcel other types of land information (e.g. utilities and administrative boundaries), the desire to transition to open source systems and have greater transparency around data custodianship and access rights, and implementation of the District Land Use Plan (DLUP) and masterplan. 
Table 2.6: Land information needs as identified by government stakeholders.

\begin{tabular}{|c|c|c|}
\hline National Level Government & $\begin{array}{l}\text { Relative } \\
\text { Importance }\end{array}$ & Popularity \\
\hline High accuracy satellite/aerial imagery & 18.7 & 0.8 \\
\hline To know who owns what spatial data & 14.7 & 0.8 \\
\hline Current land use information & 9.3 & 0.4 \\
\hline 3D cadastral data & 8.0 & 0.4 \\
\hline Utility supply data & 6.7 & 0.6 \\
\hline $\begin{array}{l}\text { Convert existing web-based system to } \\
\text { opensource }\end{array}$ & 6.7 & 0.2 \\
\hline Match land parcel to admin boundary & 6.7 & 0.2 \\
\hline Monitor operation of utilities and projects & 6.7 & 0.4 \\
\hline Integration of utility supply data & 6.7 & 0.4 \\
\hline Existing development at parcel level & 4.0 & 0.2 \\
\hline \multicolumn{3}{|l|}{$\begin{array}{l}\text { Sub-national level Government } \\
\text { (District) }\end{array}$} \\
\hline $\begin{array}{l}\text { Highly accurate spatial data (incl. } \\
\text { imagery) }\end{array}$ & 29.63 & 1.00 \\
\hline More mobile tools & 11.85 & 0.56 \\
\hline Physical characteristics of land & 11.85 & 0.44 \\
\hline Access to information & 10.37 & 0.56 \\
\hline Geological data & 8.15 & 0.33 \\
\hline Land use & 5.93 & 0.56 \\
\hline $\begin{array}{l}\text { Implementation of masterplan and DLUP } \\
\text { in an efficient way }\end{array}$ & 4.44 & 0.22 \\
\hline Parcel boundaries & 2.96 & 0.22 \\
\hline $\begin{array}{l}\text { Sub-national level Government } \\
\text { (District) cont. }\end{array}$ & $\begin{array}{l}\text { Relative } \\
\text { Importance }\end{array}$ & Popularity \\
\hline Location of underground infrastructure & 2.96 & 0.22 \\
\hline All transactions made on parcel & 1.48 & 0.11 \\
\hline Information to stakeholders & 0.74 & 0.11 \\
\hline Wireless infrastructure & 0.74 & 0.11 \\
\hline \multicolumn{3}{|l|}{ Local level Government (Cell) } \\
\hline $\begin{array}{l}\text { Spatial dataset of master plan and land } \\
\text { parcels }\end{array}$ & & 0.67 \\
\hline GIS software & & 0.67 \\
\hline Soft copy of master plan & & 0.5 \\
\hline Soft copy of the DLUP & & 0.33 \\
\hline $\begin{array}{l}\text { Integration of land use map with land } \\
\text { information database }\end{array}$ & & 0.33 \\
\hline Information about planned infrastructure & & 0.17 \\
\hline
\end{tabular}




\subsubsection{Local Government and Communities' Needs}

The local government identified similar needs, with priorities around digital data and supporting software and a related desire for land tenure information to be more readily integrated with existing or planned land use and infrastructure. In general, land use tended to accord with the purpose defined in the title, but inconsistency is starting to be an issue in areas transitioning to more urban land use types. Here, it is common for the community to be found not using their land as zoned, or in some instances, attributed to the information on the title not being updated. For example, in one Cell, despite being rezoned for urban land use (residential), some land titles still reflected the previously planned agricultural land use. In these instances, it appeared that land records were only updated if the landowner formally sought a building permit or other land-related services; otherwise, the land title remains unharmonised with the Master Plan. Also, although most of the land in Rwanda has been demarcated and titled during the LTRP, some plots (or owners who occupy the plots) remain untitled due to information gaps at the time of the LTRP e.g., lack of identification, family disputes, etc. The use of general boundaries during the LTRP for demarcation for titling has also not been updated accurately due to the resolution of GPS receivers $(3 \mathrm{~m})$ or lack of GPS receivers, leading to the on-ground practices like pacing by foot to resolve conflicts.

Lack of information, or lack of access to information, about the Master Plan (i.e., information about proposed new development) was identified. This inhibits the ability of local government to play a role in plan implementation. Additionally, given that the Master Plan plays a crucial role in setting out future development, it appears that local community consultation is ad hoc. For example, in some villages, local communities do not participate in establishing the master plan/LUP: in some others, only Cell officers are contacted, whereby it then falls onto them to inform the community; in yet others, local consultation has been undertaken.

In summary, it appears that at the Cell level, land information needs can be generalised as lack of access to development information (which affects land use practices and enforcement of intended land use types) and lack of up-to-date spatial and administrative information about individual parcels or persistent gaps in land information.

\subsubsection{Non-Government Stakeholders' Needs}

In contrast, non-government stakeholders' needs were less focused on land tenure information and more on other information needs. 
Information needs like land value, land use, history of the land, and proposed infrastructure were identified; the only tenure-related information was sub-meter accuracy of parcel boundaries in urban and peri-urban areas.

Table 2.7: Land information needs identified by non-government stakeholders.

\begin{tabular}{|c|c|c|}
\hline Non-Government & $\begin{array}{l}\text { Relative } \\
\text { importance }\end{array}$ & Popularity \\
\hline Value of land (valuation process) & 22.67 & 0.8 \\
\hline Accessible open data & 18.67 & 0.6 \\
\hline $\begin{array}{l}\text { Consultative process around land use } \\
\text { planning }\end{array}$ & 12.00 & 0.4 \\
\hline $\begin{array}{l}\text { More detailed (sub-use) land use } \\
\text { planning in Master Plan }\end{array}$ & 10.67 & 0.6 \\
\hline Actual land use information & 9.33 & 0.6 \\
\hline $\begin{array}{l}\text { History of land Information to resolve } \\
\text { conflict between infrastructure } \\
\text { development and arable land }\end{array}$ & 9.33 & 0.6 \\
\hline Integrated demographic information & 6.67 & 0.6 \\
\hline $\begin{array}{l}\text { Sub-meter accuracy of parcel } \\
\text { boundaries (urban/peri-urban) }\end{array}$ & 6.67 & 0.4 \\
\hline $\begin{array}{l}\text { Information about proposed } \\
\text { infrastructure development and } \\
\text { potential risks }\end{array}$ & 4.00 & 0.4 \\
\hline Maintained web-based Master Plans & 2.67 & 0.2 \\
\hline
\end{tabular}

It is no surprise that capacity needs around data accessibility, stakeholder engagement (e.g. consultation) and up-to-data web-based masterplans were all identified and prioritized.

\subsubsection{What Data Quality can be Achieved with UAV-Technology?}

The data quality can be derived from checkpoint residuals and a visual evaluation of the final orthomosaic. An assessment of the geometric specifications of the generated orthomosaics is outlined in Table 2.8, indicating the ground sampling distance and spatial accuracy. Almost pixel-level geometric accuracy was achieved with the Busogo dataset. Both Gahanga and Busogo show more than $10 \mathrm{~cm}$ RMS error of horizontal residuals. Differences regarding final geometric accuracy can be attributed to the UAV equipment and sensor and the device and conditions for the measurements of reference points. Nonetheless, obtained orthomosaics are of high geometric accuracy and comparable 
to results in other scientific contributions (Gerke and Przybilla, 2016; Agüera-Vega, Carvajal-Ramírez and Martínez-Carricondo, 2017).

Table 2.8: Specifications of final results.

\begin{tabular}{lll}
\hline Area & $\begin{array}{l}\text { Ground Sampling } \\
\text { Distance }[\mathbf{c m}]\end{array}$ & $\begin{array}{l}\text { RMS Error of CP Residuals } \\
(\mathbf{X} / \mathbf{Y} / \mathbf{Z})[\mathbf{m}]\end{array}$ \\
\hline Muhoza & 2.16 & $0.122 / 0.086 / 0.467$ \\
Busogo & 2.18 & $0.033 / 0.031 / 0.349$ \\
Gahanga & 2.63 & $0.127 / 0.170 / 0.244$ \\
\hline
\end{tabular}

The visual evaluation revealed commonalities but also some differences in the datasets. Figure 2.4 presents the final orthomosaics of all three datasets. Sufficient overlap was considered during the flight missions, and the reconstructed scene entirely covered the area of interest. Differences in the visual quality are evident in the close-up views. Here, the image quality was best for the Muhoza dataset, as most features, including rooftops and vegetation, were well exposed in the orthomosaic. In contrast, the Busogo dataset showed a lower image quality, visible in over-exposed roofs and problems to fulfil a proper histogram matching during image processing. This can be attributed to the adverse lighting conditions during data capture. Even though meteorological conditions were perfect for flying during data capture of the Gahanga dataset, the sensor showed substantial difficulties to deal with bright and dark image features. Especially a 
large part of the parking area is very overexposed, even though the surface was covered with reddish gravel.

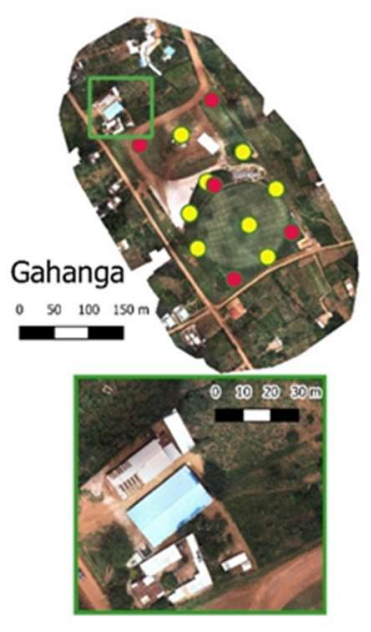

Legend

Check point

- Ground control point (GCP)

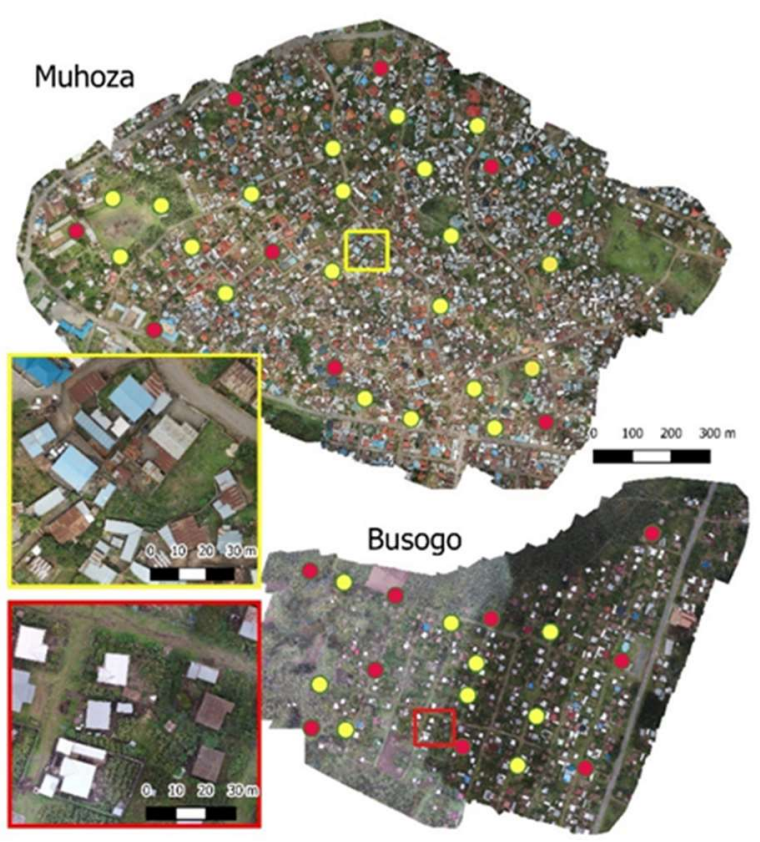

Figure 2.4: Overview of the generated orthomosaics and GCP/checkpoint distribution.

\subsubsection{Can UAV Respond to the Needs Expressed by Different Stakeholders?}

To conclude the ability of UAV data and UAV-based workflows to satisfy prioritized needs, the results of Table 2.6 and Table 2.7 were categorised and integrated with a matrix that distinguishes between (a) needs where UAV data has no significant contribution toward the achievement and (b) needs that can be matched with UAV data (Figure 2.5). The latter category was further associated with one of the four key characteristics of UAV data: high geometric accuracy, provision of up-to-date data, high spatial and temporal resolution, and a high level of interpretability. 


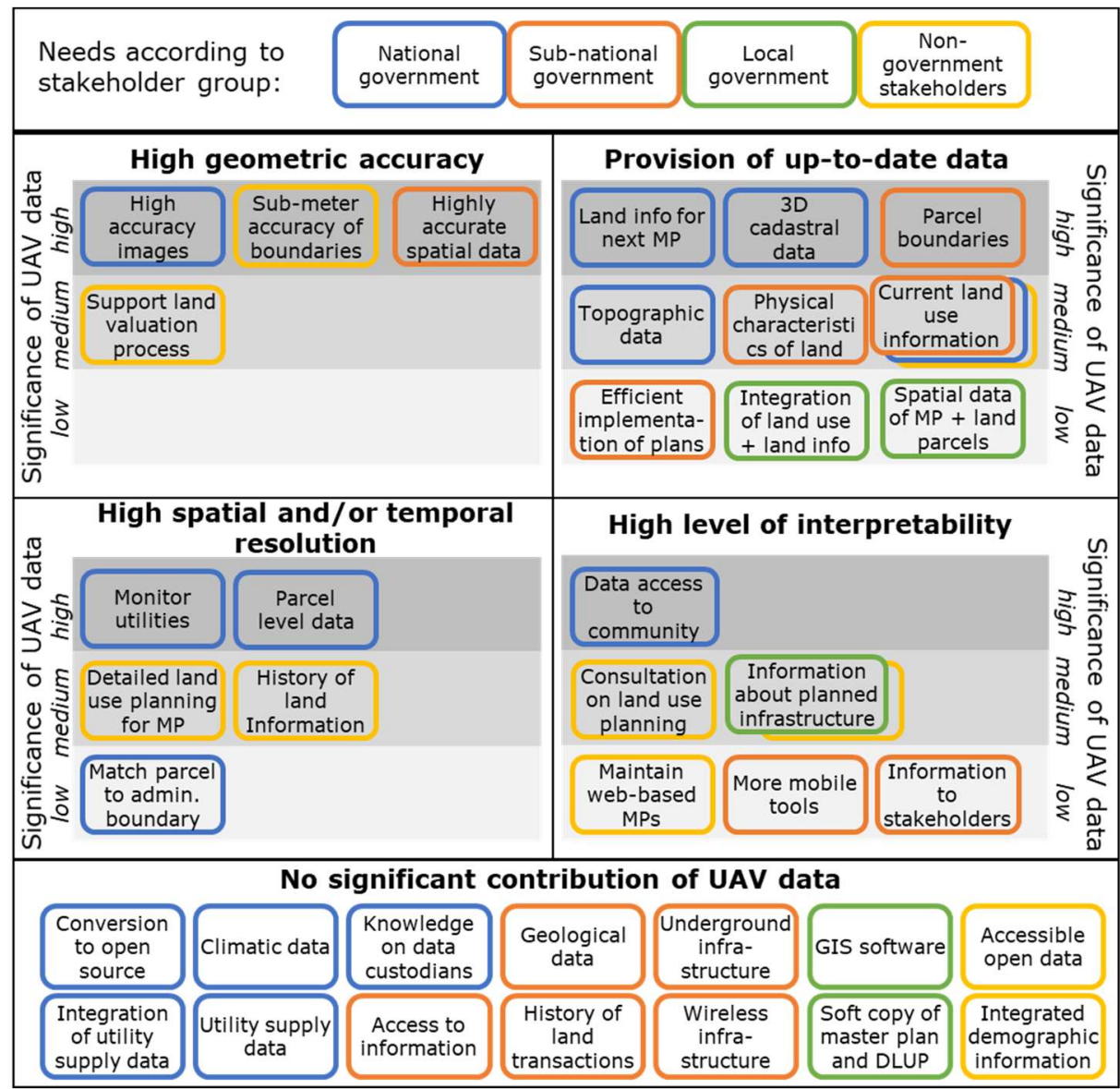

Figure 2.5: Prioritized needs classified by the ability of UAV data to match stated needs with further association to key characteristics of UAV data and ranking according to the significance of the contribution.

Figure 2.5 reveals that UAV data can significantly match 27 out of 41 prioritised needs. The remaining 14 needs mainly refer to access to data, information, and software. A high and medium significance of UAV data was primarily found among national-level stakeholders, both governmental (eight needs) and non-governmental (seven needs) organisations. The needs of the local government could be met with medium (one need) or low (two needs) significant contributions of UAV data. Most of the prioritised needs of the sub-national government can only partially be fulfilled by UAV data (i.e., medium or low significance). A comparison of the four characteristics shows that the provision of up to date data and the high level of interpretability are key in matching the stated needs. However, both aspects are highly interrelated to high geometric accuracy as well as high spatial resolution. Otherwise, the 
data would not show such a high level of detail, which leads to high interpretability and significant contribution to land use and topographic information. Although the quadrants in Figure 2.5 feature unique characteristics, they are highly interrelated and are therefore considered overlapping.

UAV regulations were found to have a considerable impact on the scale of UAV utilisation in the context of land administration. Especially flight height and line of sight restrictions limit one data collection to several tens of hectares. Mapping larger areas would thus require constant moving of the ground control station, adversely impacting the mapping efficiency. Geometric accuracy was found to be less affected by UAV regulations. In contrast, the high level of interpretability and high spatial resolutions could be an issue regarding privacy and ethical constraints. Even though not the case in Rwanda, some countries demand public consent for the data collection of private property. A condition that requires sound data collection preparations and might put significant restrictions on the UAV missions, particularly in urban and peri-urban areas.

\subsubsection{High Geometric Accuracy}

More specifically, the expressed need by government stakeholders for highly accurate spatial data can entirely be met by UAV imagery, as shown by the low RMSE of checkpoint residuals in this study. Even though the national CORS in Rwanda cannot be considered a source of GNSS corrections for PPK workflows, different means of georeferencing have proven to hit similar accuracies. This data characteristic facilitates the manual or digital delineation of parcel boundaries and supports valuation and taxation processes - two needs prioritised by nongovernment stakeholders. The current cadastral map is based on the LTR programme, which followed a general boundary approach that sometimes shows several meters offset to the correct boundary location. Most disputes arose during land transactions in densely populated areas, where plots are small, and people depend on their land for subsistence farming. In those cases, UAV data ultimately facilitates a reliable and geometrically correct database to correct existing cadastral data.

\subsubsection{Provision of up-to-Date Data}

A comparison of the obtained UAV-based orthomosaic of Muhoza and the corresponding orthomosaic, which is based on classical aerial photos from 2009 shows a high number of clearly visible changes 
(Figure 2.6), where 13 large buildings and 28 small buildings/annexes were newly constructed, 5 buildings were demolished, and 28 large buildings and 10 small buildings/annexes remained. Especially urban and peri-urban areas face numerous changes concerning development and urbanization. The high level of detail and the immediate availability of aerial photos provide the geospatial basis to extract up-to-date land use, land development, and topographic information of small scale areas, which is crucial to efficiently implementing current urban development plans. Similarly, the timely provision of UAV data could support the delineation of parcel boundaries based on orthomosaics or regular base map updating activities. Incredibly up-to-date 3D point cloud data obtained from UAV images was identified as input for the 3D cadastre. This data type can neither be provided by satellite images nor by aerial images.

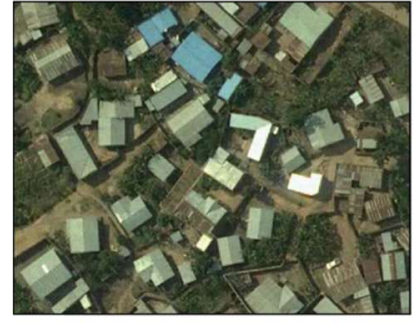

Aerial image - 2009

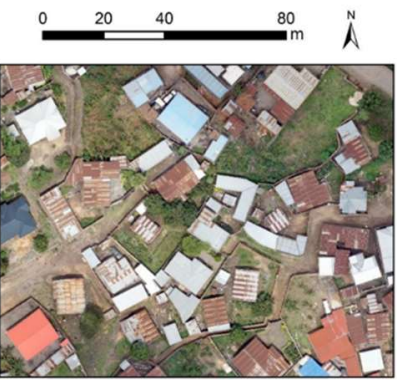

UAV image - 2018

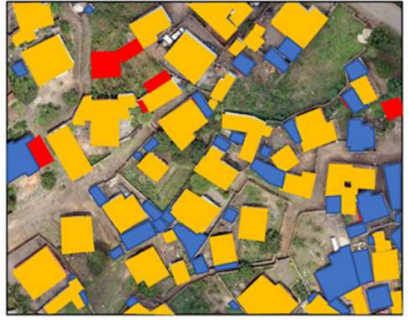

Change detection buildings

Figure 2.6: Exemplified land use changes. Left: Orthomosaic based on an aerial image from 2009; centre: Orthomosaic based on UAV images from 2018; right: Change detection of buildings (orange: buildings remained the same, red: buildings got demolished, blue: new building constructions).

A minor significant contribution of UAV data was seen in providing a multi-purpose spatial data infrastructure that enables the integration of different data, further supporting the implementation of spatial development plans. In general, UAV-based data acquisition workflows allow stakeholders to gradually upgrade existing base-maps at a small scale, without the need for significant financial outlay upfront - two fundamental aspects of fit-for-purpose approaches.

\subsubsection{High Spatial and/or Temporal Resolution}

The proven flexibility of UAV data acquisition supports the collection of multi-temporal base data for ongoing and current tasks such as the revision of the National Development Plans (i.e., Master Plans) for Secondary Cities or development plans for towns and villages. Frequent changes in land use projects can be tracked and monitored using 
repetitive UAV data collections, especially since plot sizes and administrative areas in Rwanda tend to be small and lend themselves well to UAV data capture. Additionally, disputes about former land ownership and land use can be solved more efficiently with evidence from a multi-temporal database (i.e., history of land information). Next to the temporal aspect, the high spatial resolution of UAV data allows users to extract information about developments at parcel level or a more detailed land use planning which also includes sub-uses in urban and peri-urban areas.

\subsubsection{High Level of Interpretability}

This fundamental characteristic is attributed to the high level of detail in the generated UAV products, allowing for a straightforward interpretation of the aerial dataset. People are more likely to correctly interpret the orthophoto as they recognise specific surface textures or physical features such as bushes, hedges or particular buildings. This allows UAV data to significantly enhance the (mobile) database for visualising land tenure data or planned infrastructure. This asset supports participatory mapping activities for land administration or urban planning. In many cases, the authors observed that de-jure land rights do not represent de-facto land rights as the cadastral maps show little detail on the physical extent (except for the parcel boundary). The integration of an orthophoto in the cadastral map would support the alignment of de-facto and de-jure land rights. It would spatially outline adjudicated land rights that are easy to interpret even for nonspecialists. Furthermore, UAV data could aid consultative processes of land use planning with clear and understandable background data. A profound significance of UAV data was found in support of maintaining a web-based spatial plan, promoting more mobile tools, and sharing information with stakeholders.

\subsection{Discussion}

This study was designed to determine the match between stakeholders' needs and the characteristics of the UAV data acquisition workflow and its final products as valuable spatial base data for land administration and spatial planning, particularly within the discourse of a more fit-forpurpose land administration. 


\subsubsection{Opportunities of UAV Data Collection to Match Land Information Needs}

The socio-technical assessment revealed that the technical capabilities of UAV-based data are well-placed to match most of the prioritised needs in Rwanda. These needs reflected the type of data (e.g. land use data, geological data, utility supply data, etc.) and on characteristics of data and processes (e.g. geometric accuracy, spatial resolution, custodian of data, data integration accessibility, etc.). This enabled the matching of the characteristics of UAV data to a particular type of data and the specific requirements of the data, such as temporal resolution or geometric accuracy. The synthesis, as shown in Figure 2.5, demonstrates a high number of needs where UAV data could potentially have a significant contribution. The results suggest that UAVs as a data acquisition device could most likely be adopted by national-level stakeholders or sub-national government stakeholders, which can be attributed to the system in Rwanda where the national government is the primary provider of geospatial data. However, with UAV as a lowcost and flexible data acquisition platform, sub-national or local government stakeholders could increase their share of data provision, especially concerning small scale mapping or multi-temporal flight missions in a local context. This would facilitate the co-production of land information in a decentralized way, a finding that is also reflected by (Rhamadani et al., 2018). The opportunity of using UAV-based images to delineate, update, or upgrade parcel boundaries, as exemplified in Appendix 2 of this thesis, ${ }^{3}$ is in line with the guiding principles for country implementation of fit-for-purpose land administration systems (Enemark et al., 2016). Here, UAVs were specifically emphasized as they provide large scale maps to delineate spatial units in densely populated areas (urban central, informal settlements and small towns). Results further suggest that UAV data can fulfil multiple needs across different domains such as planning and surveying. This is contrary to conventional ground surveying with GNSS or total station, where acquired data only serves a single purpose. Although not explicitly prioritised as a need, the UAV test flights showed that the (nearly) immediate availability of orthophotos could promote citizen participation in the adjudication process, a critical result also outlined by (Widodo et al., 2016; Ramadhani, Bennett and Nex, 2018). Even though the Rwandan land administration information system is very advanced in comparison to other African

${ }^{3}$ Appendix 2 reports on a practical excursus which exemplifies the use of UAV imagery for community mapping 
countries, it was found that the digital nature of a generated UAVbased orthomosaic can easily be integrated into existing spatial data infrastructures to be used by numerous GIS applications, or if absent, support the modernization of current paper-based land registration systems.

\subsubsection{Challenges of UAV Data Collection to Match Land Information Needs}

Aside from those advantages, the UAV test flights in Rwanda also reveal four main challenges about implementing UAVs as a data acquisition tool to match land information needs.

Firstly, it needs to be noted that the terrain in Rwanda - the country of the thousand hills - is a very challenging testbed. Fixed-wing drones have only limited climbing rates, and flight planning must be aligned with the physical environment. The availability of sufficient open space for appropriate landing strips is an essential precondition that was challenging to fulfil. Hybrid UAV and rotary-wing UAV are likely more suitable instruments for small scale mapping activities. Current limitations concerning battery capacity and flight time make hybrid UAVs more effective for mapping tasks as they have a better flight endurance. In contrast, a rotary-wing UAV should be preferred to monitor the operation of utilities.

The second hurdle refers to the UAV regulations in Rwanda. With an operational limitation to fly only in visual sight, scaled application of UAV-mapping activities remains aspirational. Acknowledging the plans of the Government of Rwanda, legislation with a more performancebased orientation may soon be drafted and implemented more effectively. This development could pave the way for broader use of UAV-based data acquisition that supports land tenure recording and extensive land information collection for development purposes (Union African and NEPAD, 2018).

The third hurdle includes the topic of ground-truthing. It has been shown that the collection and measurement of reference points are challenging, especially in an urban environment, and ground marking should be context-specific. PPK and RTK capable UAVs can provide an answer to this challenge as they minimise or even eliminate the need for ground control measurements. However, the availability of professional GNSS equipment or a national network of existing GNSS reference stations is an essential precondition for RTK or PPK-based workflows. If the national CORS is unreliable or nonexistent, other 
means of accurate GNSS measurements such as Precise Point Positioning should be considered.

The fourth challenge refers to soft- and hardware requirements for data processing. The authors' experiences in Rwanda revealed that the majority of employees of the Rwanda Land Management and Use Authority have a machine that could process smaller datasets up to 500 images. However, to facilitate the processing of the data of an entire township, devices with more RAM and disk space would be needed - ideally, a server environment. Cloud-based processing is seen as very critical, as internet connections are very often subject to outages. Financial barriers to purchase the required hardware and commercial software such as Pix4D or similar were perceived as very high - costs that are likely to exceed the procurement costs of the UAV equipment. At the same time, current open source software cannot reproduce the same data quality as commercially available software. However, given the rapid development of Open Drone Map, and the increasing number of users, the software algorithm is likely to mature soon.

\subsubsection{Limitations of this Research and Future Work}

The scope of this study was limited regarding the validation of the UAV technology in an operational environment as UAV flights were only trialled without the direct implementation of the outcomes. Thus, this study did not address gaps and challenges on how the expressed stakeholder needs were met in the context of Rwanda. Future work could address this with a strong focus on implementation to evaluate the fitness-of-use of UAV workflows with due consideration of the entire innovation chain, including GIS applications. This could be coupled with the design and evaluation of appropriate governance and capacity building models to allow the prototype demonstration of UAV-based workflows in an operational environment. Additionally, further research will be needed to explore the role of UAV compared to other geospatial technologies such as satellite data and classical aerial photographs in providing base data that serves as a spatial framework for the various land administration functions (Enemark, 2004).

\subsection{Conclusion}

The presented work highlights the capabilities of UAV technology to match the needs of land professionals in Rwanda. Results of a sound needs assessment across different stakeholder groups demonstrate the prioritised demands of respective respondents. Although ranked 
differently, the need for high-resolution, up-to-date land information is consistently identified in the final lists of all group discussions. Across the globe, UAVs have become an attractive technology, and only the upcoming years will show whether multiple governmental and nongovernmental stakeholders can capitalise on the numerous benefits of this data acquisition method. The flight missions in Rwanda showed that UAV as a platform to remotely capture images have clear advantages in terms of fit-for-purpose data provision by facilitating accurate, up-to-date data with a potentially high spatial and temporal resolution. However, the integration of the needs assessment and the UAV test flights indicates that structural and capacity conditions currently undermine the vast potential of the UAV data acquisition method. Therefore, a key policy priority should be to implement country-specific capacity development and governance strategies; otherwise, scaled implementation and increasing technology uptake might remain wishful thinking. Notwithstanding the outlined challenges, the results of this study show that UAV technology can be an appropriate land tool with a significant contribution in catering the base data for most of the prioritized land information needs in Rwanda. 


\section{Chapter 3 - Legal Considerations for Applying UAVs in Land Administration ${ }^{4}$}

4 This chapter is based on:

Stöcker, C., Bennett, R., Nex, F., Gerke, M., \& Zevenbergen, J. (2017). Review of the current state of UAV regulations. Remote sensing, 9(5), 459.

Stöcker, C., Zevenbergen, J. (forthcoming). Legal Considerations of UAV flights. In: A. Eltner, D. Hoffmeister, A. Kaiser, P. Karrasch, L. Klingbeil, C. Stöcker, A. Rovere (Eds.). UAVs for the Environmental Sciences. wbg Academic. 


\subsection{Introduction}

Recent technological improvements and increasing operational capabilities present specific challenges to flight operators, end-users and aviation authorities: especially concerns about privacy, data protection, and public safety (Finn and Wright, 2014). To minimize the risks of UAV-triggered incidents or accidents, an increasing number of national and international authorities have introduced legal provisions that mandate "Go," "No go", or "How to go" statements that either allow, prohibit or restrict flight operations. Such regulations significantly impact how, where, and when data can be captured-and the diffusion of the technology within a national context.

Due to the rapid emergence of this technology, UAV regulations are still embryonic, and heterogeneity of national rules and varying levels of implementation can be observed (Watts, Ambrosia and Hinkley, 2012; van Blyenburgh, 2016). The CEO of the AscTec UAV company stresses that "legislation and policymaking are lagging way behind the technology" (van Wegen and Stumpf, 2016). This lack creates a significant barrier to research and development as scientific projects are hindered (Watts, Ambrosia and Hinkley, 2012), both private and public innovation. As a result, market opportunities and societal gains are not being exploited (Boucher, 2015). Common problems with UAV regulations include flight approval times (Rango and Laliberte, 2010) and poorly documented administrative processes that limit the desired flexibility and impede the widespread utilization of the technology. However, some national aviation authorities and international organizations are already moving to "modernize" the first wave of regulations: they seek to accommodate user demands and recent technological developments while still maintaining safe operations.

Motivated by a lack of a sound overview of the recent developments of UAV regulations, this chapter highlights the importance of UAV regulations as one prerequisite that dictates when, where, under which conditions and by whom data can be captured. Furthermore, it will help better understand the impact of UAV regulations on the remote sensing community as this review unveils the practical limitations of UAVs as a remote sensing platform and the need for improved technical solutions. This chapter aims to provide an exploratory investigation of UAV regulations on a global scale. This includes a global overview and a thorough discussion of the main mandated criteria in the regulations. Next, we particularly highlight time as an additional dimension to distinguish past, present and expected future developments. 
To this end, and to underline the urgency of the work, the following section presents the background to contemporary socio-technical perspectives on UAV regulations. Materials and methods, including the data utilized and analysis techniques applied, are then outlined. The results section presents the status of UAV regulations considering past, present, and future trends. Similarities and contrasting elements in various national UAV regulations and their implications for data acquisition activities are explored. In addition, the work investigates and discusses current considerations of privacy, data protection and public safety within the legal frameworks for operating UAVs. In considering the scientific discourse on "law and technology" and the current state of UAV regulations, possible future trends are outlined. The discussion section aims to place the results into the state-of-theart context and show the limitations of the data utilized and applied methods in this chapter. Finally, conclusions are drawn, and directions for future work are suggested.

\subsection{State of the Art}

Before the paragraph proceeds with vital background information, it is necessary to spend a few lines on the definition of UAVs. Although UAV is the most well-known term in both professional and non-professional domains, regulatory bodies like the International Civil Aviation Authority (ICAO) emphasize clear distinctions between UAVs-these are of particular interest for the applicability of laws and regulations. According to (ICAO, 2015), the broad group of UAVs can be distinguished to remotely piloted aircraft (RPA) and autonomous aircraft. Here, the latter refers to UAVs that do not allow pilot interventions during the flight and are mainly used in military contexts. The former refers to unmanned aircraft which are remotely controlled by a pilot (ICAO, 2015). RPA is used mainly for civil applications. Model aircraft represent the third group and are defined to be solely intended for fun and recreational purposes. As the ICAO terminology is not yet implemented in all countries, this terminology was used differently. However, the following sections only acknowledge regulations, statements and content that focus on civil applications.

UAV regulations have already been mentioned in various publicationsa refined Scopus literature search found more than 300 publications (conference paper, articles, reviews ...) from 2000 onwards. However, authors of relevant articles either discuss the topic from one context (e.g., privacy), technical specification (e.g., sense and avoid systems), or cover only a few countries. 
Although the legal provisions are one important part of the preparation phase of UAV data acquisition flights, most technically or applicationoriented articles do not specifically mention the regulations that applied to their data collection flights (Watts, Ambrosia and Hinkley, 2012). Some review articles on UAV applications discuss UAV legislative frameworks: they present national and international regulatory bodies and give short introductions to risk-based approaches, UAV safety level classifications and current efforts of international organisations to harmonize UAV legislation (Remondino et al., 2011; Zhang and Kovacs, 2012; Colomina and Molina, 2014). However, the works provide only brief overviews and remain highly generalized. In this context, the Federal Aviation Administration (FAA-US) regulations are exceptional and need to be emphasized in particular as they are subject to dedicated research articles (Rango and Laliberte, 2010; Watts, Ambrosia and Hinkley, 2012). The authors stress the impact of regulations for UAV operations in the US and highlight safety procedures that need to be followed. They further indicate the desired future situation that mainly concerns the extension of visual line-ofsight flights and simplified administrative procedures. In comparing six different national regulations, (Morales, Paez and Arango, 2015) evaluated various options for Colombian regulatory frameworks.

In contrast to technically or application-oriented articles, scientific publications with a social-technical background mainly focus on societal aspects. Here, the discourse of UAV regulations includes public safety and liability in case of an accident, societal benefits and stakeholders in international regulatory bodies such as the European Commission (Boucher, 2015), rule-guided risks and opportunities of UAV (Villasenor, 2013), ethical tolerability (Jansen, 2015), and effects upon privacy and other civil liberties (Finn and Wright, 2012). The latter mentioned authors further used self-reported material of representatives of different drone sectors (including civil aviation authorities and data protection authorities) to study perspectives on privacy, data protection and ethical aspects (Finn and Wright, 2016). They detected "[...] significant gaps in the industry's knowledge about their privacy, data protection and ethical obligations under European and national laws" and that the legislation regarding these concerns lacks tremendously (Finn and Wright, 2016). Interestingly, (Boucher, 2015) showed another example of non-compliance. The authors conclude that the strategies to manage public acceptance in the European roadmap (European RPAS Steering Group, 2013) do not conform with the European concept of responsible research and innovation (European Commission, 2016). 
Furthermore, in a series of papers, (Clarke, 2014) identified the risks and drawbacks of the utilization of UAVs. Among others, he mainly raises the topic of air safety laws and summarizes national as well as international legislation as incomplete due to "[...] inadequate and very slowly-adaptive formal regulation" (Clarke and Bennett Moses, 2014).

The literature review reveals that although the methodologies of application-oriented articles differ from those with socio-technical backgrounds, the subject of interest is, in many cases, the same-e.g., FAA regulations. However, very few cross-citations from one domain to the other were noted.

Lobby groups, including UVS International, are pushing towards regulatory standards and harmonization. International roadmaps as in Europe (e.g. European RPAS Steering Group, 2013) anticipate a future with full integration of civil UAVs into national aviation systems. In contrast to these prospects, lawyers and social scientists emphasize privacy, data protection and public safety concerns and identify significant gaps in current regulatory frameworks. At the same time, emerging and fast-developing technologies such as UAVs often involve legal problems (Bennett Moses, 2007) due to inflexibility and slow adaptation processes of formal laws. The multitude of evidence, perspectives, and visions of scholars and various stakeholders alike, let alone (sometimes inappropriate) media reports, make it hard to draw a clear picture of past developments, current status and future trends of UAV regulations. Thus far, to the best of the authors' knowledge, no scientific publication comprehensively analyses different statuses of UAV regulations. In response, this chapter seeks to develop a holistic perspective by synthesizing approaches, concepts and ideas gained from a comprehensive analysis of civil UAV regulatory frameworks and recent scientific literature from the fields of law, innovation, and technology.

\subsection{Data Sources and Methods}

The overall methodology encompasses a research synthesis of multiple data sources related to UAV governance, legislation, and regulatory frameworks. In general, a research synthesis uses existing facts and numerous references to generalise the research topic (Cooper and Hedges, 2009). It thus fits the aim of providing a comprehensive overview of UAV regulations and their implications for flight operations. The first methodological pillar of the research synthesis is a comparative analysis of the various documents that regulate UAV operations. This analysis embraces national regulatory frameworks, 
international principles and guidelines, which are analysed comparatively. Facilitated by a range of variables, a point-by-point comparison allows for both quantitative and qualitative analysis. The variables are assigned to a set of six criteria that consider the main aspects of UAV regulations. Criteria and respective variables were derived following a heuristic approach based on an iterative review process of legal frameworks for UAVs. Hence, the findings provide an overview of the characteristics of both past and present UAV regulatory approaches and enable predictions of future trends. In addition, a review of the scientific literature that focuses on the relationship between law, innovation, and technology constitutes the second pillar of the methods for this research synthesis. The literature is reviewed from the point of view of legal considerations of UAV regulations and lessons learned from other "problems with 'technology' as a regulatory target" (Bennett Moses, 2013). Outcomes will provide further inputs for the prediction of possible future trends. The framework for this analysis is outlined in Figure 3.1.

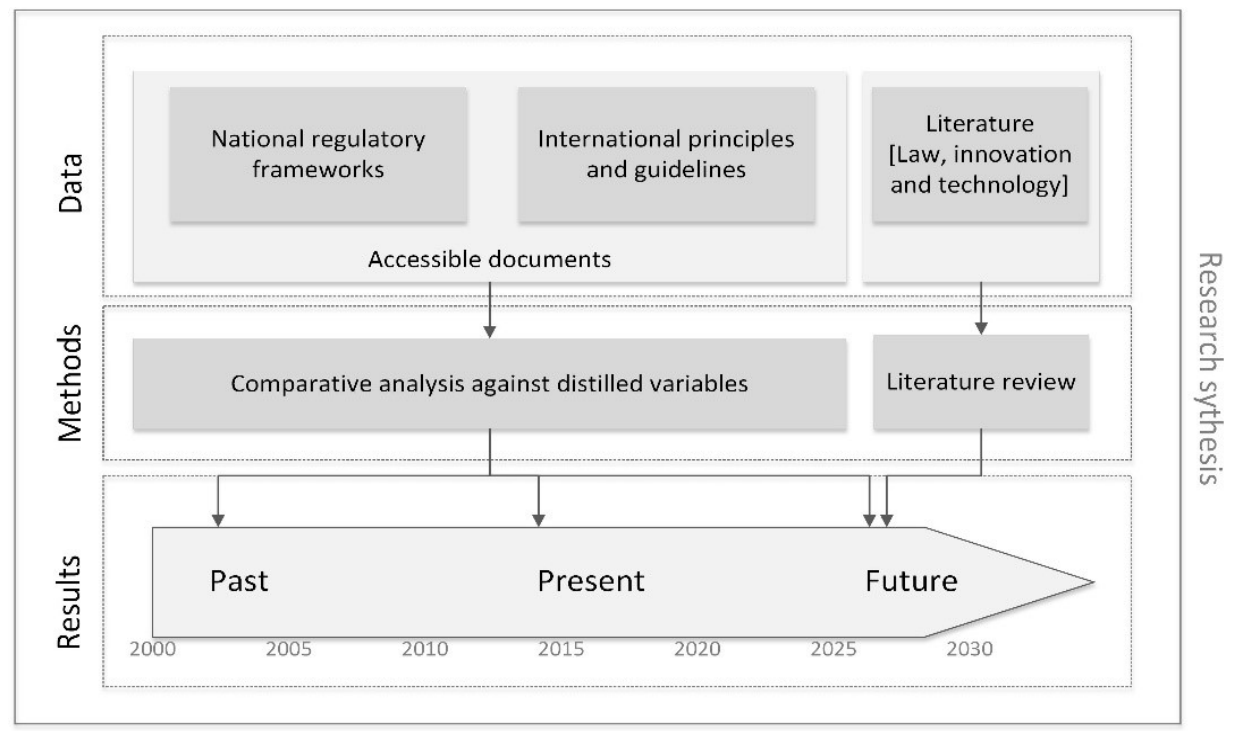

Figure 3.1: Conceptual framework of chapter 3.

\subsubsection{Database}

The search strategy and selection criteria need to be explicitly outlined to ensure an objective and reproducible database. The first step of the search strategy targets national UAV regulations and involves a comprehensive online search. Due to local language constraints, an online search of regulatory documents country by country would be 
impractical for this project. Thus, internet sources of relevant international UAV organizations are reviewed for pre-compiled lists and overviews. Table 3.1 presents a list of known sources that link national UAV regulations and briefly outlines their content.

Table 3.1: Overview of online-accessible lists and overviews of UAV regulations (status October 2019)

\begin{tabular}{|c|c|}
\hline Internet Presence & Content \\
\hline http://jarus-rpas.org/regulations & $\begin{array}{l}\text { Joint Authorities for Regulation of } \\
\text { Unmanned Systems (JARUS): List } 30 \\
\text { national UAV regulations and provide } \\
\text { a detailed comparison }\end{array}$ \\
\hline https://droneregulations.info & $\begin{array}{l}\text { Collaborative wiki: Global UAV } \\
\text { Regulations Database which is } \\
\text { comprised of a country directory with } \\
\text { summaries of national drone laws, } \\
\text { links to original regulatory documents } \\
\text { and additional resources. }\end{array}$ \\
\hline https://dronerules.eu & $\begin{array}{l}\text { Co-funded by the European Union, } \\
\text { the portal informs about the basic } \\
\text { requirements and applicable drone- } \\
\text { related laws and regulations across } \\
\text { the EU, Norway and Switzerland. }\end{array}$ \\
\hline
\end{tabular}

Due to the rapid emergence of and ongoing changes to UAV regulations, none of the collections provides a reliable, complete and coherent picture of the worldwide situation. Links, documents and information are either outdated, incomplete or still in development and therefore not yet released. Thus, this chapter used all links available from these platforms for a global overview of UAV regulations.

Based on this information, a sub-sampling of regulations for a deep and detailed comparative analysis was realised. Consequently, the representative sample of 19 regulations covers all continents and a diverse range of legal systems, economic development levels, and geographical environments. Furthermore, various times of the first release were acknowledged to identify both pioneers and followers. Data sources include principles, guidelines, codes of conduct and roadmaps. Oral communications and references from the grey literature provided an additional valuable source of information. 


\subsubsection{Methods}

A comparative analysis is a very general research method (Lijphart, 1971): it compares two or more cases and thus puts a particular sample of subjects into a relation. The rationale behind a comparative analysis in the context of UAV regulations is to aim for a narrative of developments, commonalities, and differences in various regulatory documents. Measurable variables need to be determined to achieve a quantitative point-by-point analysis rather than a qualitative case-bycase comparison (UNC, 2003).

Twenty variables were distilled in a heuristic approach by an iterative review and comparison of UAV regulations. In our case, all variables are dichotomous (Kumar, 2014) and can show one of two characteristics: addressed or not addressed by respective UAV regulations. In addition to this, the analysis includes descriptive information of the majority of variables to improve their significance and highlight the qualitative variations among specific characteristics.

As shown in Table 3.2, six main criteria and respective variables were chosen. (1) Applicability refers to the scope of respective regulations. The first two variables distinguish regulations' applicability to model aircraft (MA) or UAVs in this group. Furthermore, applicable classifications and weight limits are addressed by the latter two variables. (2) Technical prerequisites acknowledge essential instruments that are mandated. Referring to current technological developments, collision avoidance capabilities are treated as an individual variable. (3) Operational limitations cover restrictions for the flight itself and include the following variables: distances to airports/airstrips and people, limitations to operating over congested areas, acknowledgement of prohibited areas, additional limitations, maximum flying height, and limitations regarding the visibility of the UAV. (4) Administrative procedures refer to visits to authorities as well as required documents and services. In this context, the application procedure, the need for registration and the required insurance cover are separate variables. (5) Human resource requirements encompass the piloting skills mandated. (6) Implementation of ethical constraints refers to the inclusion of references to privacy and data protection regulations.

This comparison builds the base of the discourse regarding pioneering countries and the identification of possible trends. The outcomes will be reflected towards the current mandates of international organizations and lobby groups. 
Table 3.2: Overview of criteria and variables of the comparative analysis.

\begin{tabular}{|c|c|}
\hline Criteria & Variable(s) \\
\hline Applicability & $\begin{array}{l}\text { Applicable for model aircraft (MA), applicable } \\
\text { for UAVs, classification, weight limits }\end{array}$ \\
\hline Technical requirements & $\begin{array}{l}\text { Required instruments, required level of sense } \\
\text { and avoid mechanism }\end{array}$ \\
\hline Operational limitations & $\begin{array}{l}\text { Distance to airports/strips, limitations to fly } \\
\text { over people, restrictions over congested areas, } \\
\text { prohibited areas, additional restrictions, } \\
\text { maximal flying height, visual line-of-sight, } \\
\text { beyond visual line-of-sight }\end{array}$ \\
\hline $\begin{array}{l}\text { Administrative } \\
\text { procedures }\end{array}$ & $\begin{array}{l}\text { Application procedure and operational } \\
\text { certificate, need for registration, need for } \\
\text { insurance }\end{array}$ \\
\hline $\begin{array}{l}\text { Human resource } \\
\text { requirements }\end{array}$ & Qualification of pilots \\
\hline $\begin{array}{l}\text { Implementation of ethical } \\
\text { constraints }\end{array}$ & $\begin{array}{l}\text { Indication of requirements for data protection, } \\
\text { Indication of requirements for privacy }\end{array}$ \\
\hline
\end{tabular}

\subsection{Analysis}

As UAVs are a new object in the airspace, they pose a potential risk to other airspace users and third parties on the ground. Therefore, a growing number of countries are establishing regulations to minimize this risk. The results reveal that UAV regulations are subject to national legislation and focus upon three key aspects: (1) targeting the regulated use of airspace by UAVs as they pose a danger for manned aircraft; (2) setting operational limitations to assure appropriate flights; and (3) tackling administrative procedures of flight permissions, pilot licenses and data collection authorization.

As presented in Figure 3.2, the global overview of UAV regulations as of October 2019, more than $50 \%$ of all nations have documents containing specific instructions for the use of UAVs. Most of these documents refer to regulations enforced by law, whilst a few countries published only guidelines or public notices as the law-making process is still in progress. In 2019, six nations banned the use or even the import of UAVs (Kenya, Egypt, Uzbekistan, Brunei Darussalam, Cuba and Morocco). Approximately one-third of all countries do not provide any information regarding the use of UAVs for civil applications. However, this does not imply that flights are per se prohibited or allowed. Announcements for pending UAV regulations were found in 8 countries. In 13 cases, the information of relevant precompiled lists 
could not be validated, and no documents were found proving the existence of particular regulations.

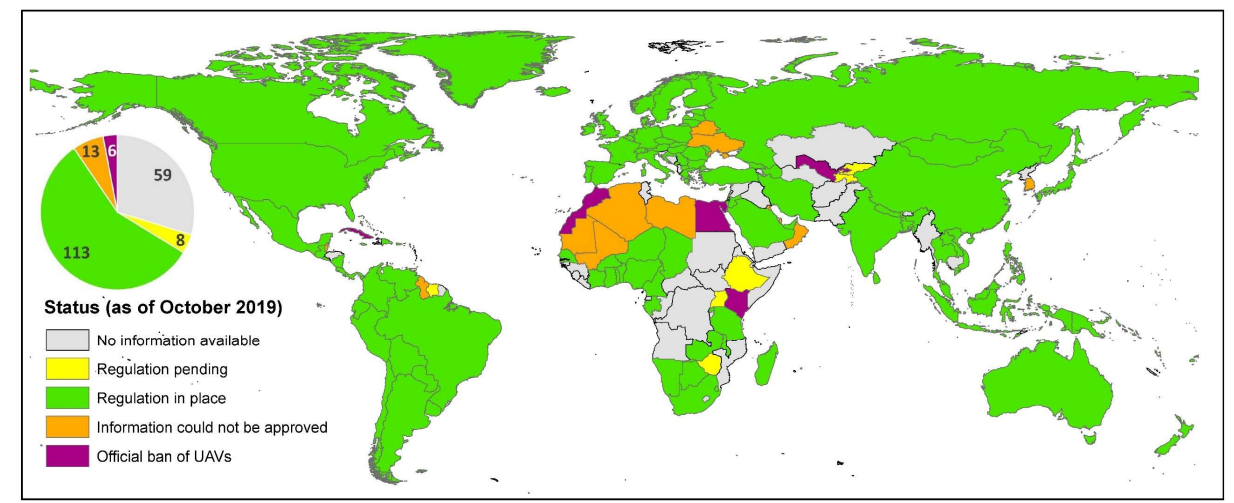

Figure 3.2: Global overview of the current status of UAV regulations on a country-level resolution (status: October 2019, based on Stöcker et al. 2017).

The following subsections cover past developments and highlight present states of application both in national and international contexts.

\subsubsection{Past}

The history of UAV regulations dates back to manned aviation and the emergence of aeroplanes during World War II. In 1944, the international community established the first globally acknowledged aviation principles - the Chicago Convention. Besides the main focus on requirements for safe and secure flights in manned aviation, one article addresses pilotless aircraft and highlights the need for special authorization of UAV operations.

"No aircraft capable of being flown without a pilot shall be flown without a pilot over the territory of a contracting State without special authorization by that State and in accordance with the terms of such authorization. Each contracting State undertakes to ensure that the flight of such aircraft without a pilot in regions open to civil aircraft shall be controlled as to obviate danger to civil aircraft."

\section{Article 8 (ICAO, 1944)}

Due to the early developments of UAVs in manipulated model aircraft (Eisenbeiß et al., 2009), UAV operations were usually conducted under respective regulations for model aircraft (Rango and Laliberte, 2010). In the 2000s-after years of technological research and innovation- 
UAVs developed into a commercially workable system. Hence, in 2006, the ICAO identified and declared the need for international harmonized terms and principles of the civil use of UAVs (ICAO, 2015).

To strengthen the operation of UAVs throughout the world in a safe manner, ICAO published Circular 328 AN/190 in 2011 as a first step to provide a fundamental international regulatory framework through Standards and Recommended Practices. In 2016, the same organisation published an online toolkit that delivers general guidance for regulators and operators. ICAO further issued recommendations for the safe integration of UAVs into controlled airspace. In those, UAVs are "envisioned to be an equal partner in the civil aviation system [that is] able to interact with air traffic control and other aircraft on a realtime basis" (ICAO, 2015). As this manual mainly focuses on the global harmonisation of UAV operations in air traffic-controlled environments, lower priority is granted to visual line-of-sight (VLOS) operations (ICAO, 2015).

At a national level, the UK and Australia were the first nations that promulgated regulations in 2002. Some European countries and the US, Canada, Brazil, and Russia followed during the next years. As visualised in Figure 3.3, most countries - particularly in Asia and Africa - remained without regulations during that time. Only after 2012, aided by guiding documents of the ICAO and a continually growing UAV market, the number of countries with enacted UAV rules and regulations increased significantly.

Generally speaking, national UAV regulations were established due to the growing UAV industry and the identified need to regulate the emerging technology for public safety. In several cases, countries further promulgated regulations to respond to high-profile incidents, as witnessed in Japan. Here, UAVs have initially been widely operated without a sophisticated regulatory framework. However, an incident where a UAV that carried radioactive soils and intentionally landed on the rooftop of the Prime Minister's office triggered the discussion and the subsequent revision and amendment of the Japanese Aviation Act (The Japan Times, 2016). 


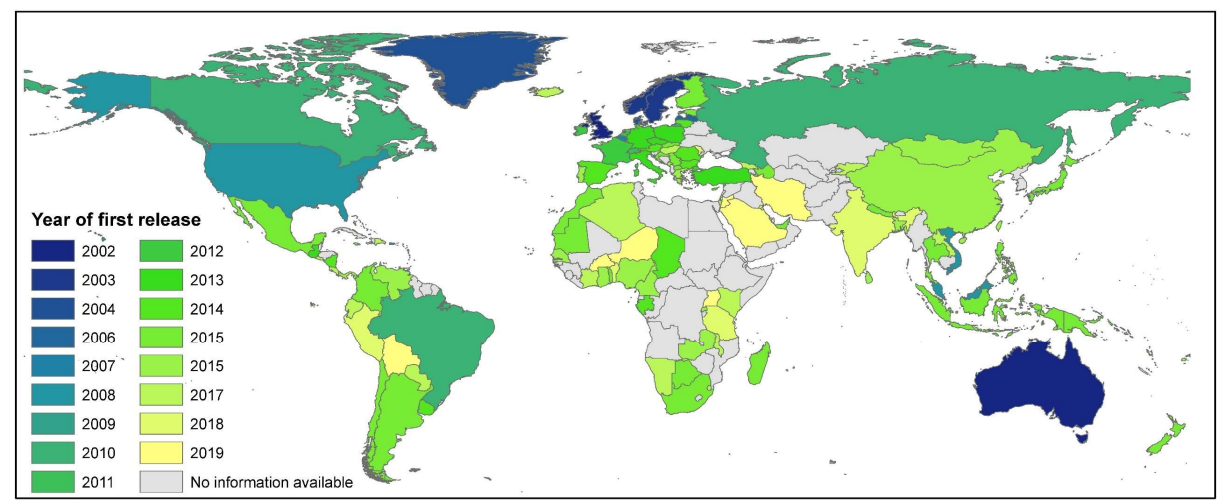

Figure 3.3: Global distribution of first releases of UAV regulations on country level resolution (status: October 2019, based on Stöcker et al. 2017).

Besides national efforts to introduce UAV regulations, international organizations took initiatives in parallel. In 2002, the Joint Aviation Authorities (JAA) and European Organization for the Safety of Air Navigation (EUROCONTROL) jointly established a UAV Task Force that aimed at safely integrating UAVs into European Airspace through the setting out of a guiding concept for civil UAV regulations (Joint Aviation Authorities and Eurocontrol, 2004). As a successor of the JAA, the European Safety Aviation Authority (EASA) further pursued this mission from 2008 onwards. Various documents for the development of one European policy on UAVs were published. In 2012, the European Commission set up the European RPAS steering group (ERSG)-a gathering of organizations and experts in this field. ERSG received the mandate to create a roadmap for integrating civil UAVs into the European aviation system (European RPAS Steering Group, 2013).

Next to the regional context of Europe, global interest groups and professional organizations such as the Joint Authorities for Rulemaking on Unmanned Systems (JARUS)-a particular UAV study group of ICAO-evolved as well. The most relevant documents are outlined in the next subsection, following a brief description of these international organisations.

\subsubsection{Present}

\subsubsection{International Context}

The ICAO is an international actor that serves as a collaboration and communication platform for national civil aviation authorities. They are concerned with fundamental regulatory frameworks globally and 
provide information material, Standards and Recommended Practices with supporting Procedures for Air Navigation Services (ICAO, 2011).

JARUS is a group of 49 national authorities (12/2016) and experts. Founded in 2012, this international actor aims for harmonized standards and provides guidance material to support and facilitate the creation of national UAV regulations. In particular, they recommend operational, safety and technical regulations that notably focus on UAVs that weigh less than $150 \mathrm{~kg}$. Amongst others, current publications include detailed recommendations for light unmanned rotorcraft systems (JARUS, 2013), requirements for C2 command and control link (JARUS, 2016) and recommendations for personnel licensing (JARUS, 2015). Ongoing work concerns design objectives for detect and avoid systems and operational categorizations.

Besides global acting organizations, diverse stakeholders in the European Union discuss developments and principles for future regulatory frameworks of UAVs. One crucial step includes the publication of the Riga Declaration on Remotely Piloted Aircraft (EASA, 2015). This declaration highlights five main principles that should guide the regulatory framework in Europe: (1) Drones need to be treated as new types of aircraft with proportionate rules based on the risk of each operation; (2) EU rules for the safe provision of drone services need to be developed now; (3) Technologies and standards need to be developed for the full integration of drones in the European airspace; (4) Public acceptance is key to the growth of drone service; (5) The operator of a drone is responsible for its use (EASA, 2015).

Regulation of UAVs below $150 \mathrm{~kg}$ was handled by all member states individually until August 2018. With Regulation (EU) 2018/1139, the European Commission received the order to regulate all sizes of UAVs (European Parliament, 2018). Following its mandate, EASA published the first common European UAV rules in Summer 2019, which has come into effect as of January $1^{\text {st }} 2021$ and will replace existing national provisions (European Commission, 2019). Ultimately, this regulatory reform allows to harmonise the European UAV market and enables UAV pilots to easily accomplish UAV flights in the EU without struggling with heterogeneous national legislation. While aiming primarily to ensure safe operations of UAVs, the European regulatory framework will also facilitate the enforcement of citizens' privacy rights and contribute to addressing security issues and environmental concerns. The current approach is risk-based (3.2.1) and distinguishes three main categories applicable for commercial and recreational users alike: the low risk "open category", the "specific category", and the 
high risk "specific category". Specifications are outlined in Figure 3.4. In this scheme, European and national aviation authorities (NAA) share responsibilities for authorisation. The regulations are planned to be fully implemented by national aviation authorities by January 2023. The U-Space concerns a Unmanned Traffic Management System and includes services and procedures to manage the safe operation of UAVs in various airspace classes.

\begin{tabular}{|c|c|c|c|}
\hline Category of operations & $\begin{array}{l}\text { Open } \\
\text { low risk }\end{array}$ & $\begin{array}{c}\text { Specific } \\
\text { medium risk }\end{array}$ & $\begin{array}{l}\text { Certified } \\
\text { high risk }\end{array}$ \\
\hline Authorisation needed & None & $\begin{array}{l}\text { Authorisation from NAA based } \\
\text { on operational risk assessment } \\
\text { or specific scenario }\end{array}$ & $\begin{array}{l}\text { Authorisation from } \\
\text { NAA/EASA }\end{array}$ \\
\hline UAS & $\begin{array}{l}\text { Compliant with Commission } \\
\text { Delegated Regulation on UAS }\end{array}$ & $\begin{array}{l}\text { Compliant with requirements } \\
\text { included in the authorisation }\end{array}$ & Certified UAS \\
\hline Operations allowed & $\begin{array}{l}\text { Restricted to: } \\
\text { - VLOS } \\
\text { - } \quad \text { Altitude }<120 \mathrm{~m} \\
\text { - Other limitations defined } \\
\text { by: } \\
\text { - Commission } \\
\text { Regulation on UAS } \\
\text { operations } \\
\text { - National airspace } \\
\text { zones }\end{array}$ & $\begin{array}{l}\text { Restricted to: } \\
\text { - Operations specified in } \\
\text { the authorisation } \\
\text { - Limitations defined by } \\
\text { national airspace zones }\end{array}$ & $\begin{array}{l}\text { Controlled airspace } \\
\text { U-Space }\end{array}$ \\
\hline \multirow[t]{2}{*}{ Regulations } & \multicolumn{2}{|c|}{ Commission Regulation on UAS operations in open and specific } & $\begin{array}{l}\text { Revision of existing } \\
\text { aviation regulation }\end{array}$ \\
\hline & $\begin{array}{l}\text { Commission Delegated } \\
\text { Regulation on UAS }\end{array}$ & $\begin{array}{l}\text { No regulatory requirement } \\
\text { (UAS requirements included in } \\
\text { the authorisation) }\end{array}$ & \\
\hline
\end{tabular}

Figure 3.4: Categorisation of UAV operations according to Commission Implementing Regulation 2019/947 (source: Dronerules.eu)

Next to governmental efforts on national and international levels, UAViators, Humanitarian UAV Network refers to a worldwide initiative with more than 2500 members. Besides other tasks, this initiative developed clear standards for the responsible use of UAVs. Documents and databases are the results of a collaborative action of active members. Their code of conduct was drafted in an open consultative process involving UAViators members, UAV experts, and global acting organizations (UAViators, 2015).

\subsubsection{National Context}

The detailed comparative analysis reveals an apparent heterogeneity of national UAV regulations as of October 2016. Except for the variable that addresses VLOS and the lateral distance of the pilot, none of the 
remaining 19 variables shows any widespread accordance across all cases. However, clusters and similarities between countries become visible as well. The following paragraphs provide a summary of each criterion, highlighting generalities and particularities before certain country-specific contexts and constellations are shown. Specifications are outlined in Appendix 3.

\section{Applicability}

In general, UAV regulations only apply for specific scenarios of civil UAVs that are classified and limited by the weight of the UAV and the area, operational range or purpose of its utilization. Most countries define commercial flight operations as flights for purposes other than just for the flight itself. Here, a mounted camera can already indicate commercial use (e.g., Austria). However, 15 countries also include the regulations of UAVs for fun and recreational purposes. More than half of the countries do not distinguish any purpose and thus have both uses. Only four countries explicitly exclude UAVs for non-commercial uses. This initial definition of the applicability is further reflected in different safety levels for respective uses. The same applies to another classification criterion: weight. All countries except Japan, China, Rwanda and Nigeria have a classification scheme according to the weight and thus follow the basic concept of a risk-based approach-the higher the associated risk (i.e., weight), the stricter the flight conditions.

Regarding weight limitations, only two countries-U.S. and Japanintroduce a minimum threshold: $250 \mathrm{~g}$ and $200 \mathrm{~g}$, respectively. All drones that are lighter than the threshold are not subject to the regulations. In contrast, almost all countries incorporate a maximum take-off mass (MTOW). UAVs heavier than $150 \mathrm{~kg}$ are usually not regulated by national aviation authorities but by international bodies like EASA in Europe. This group of UAVs is generally handled similarly to manned aircraft (EASA, 2016). However, the MTOW is not consistent in all cases. Here, China and Chile are extreme cases that allow comparatively low MTOWs. Other predominant weight thresholds refer to $20 / 25 \mathrm{~kg}$ and $\leq 10 \mathrm{~kg}$. The presence of weight classes allows for multi-layered regulations that are adopted to the associated risk. This approach allows for further differentiations regarding administrative procedures and requirements for pilot qualifications.

Next to the classification according to the weight, Austria, France, and Italy include the intended flight area as a second criterion. Consequently, these countries create different scenarios compiled in a 
more complex risk matrix (cf. Austria). France and South Africa also incorporate visibility in their scheme. Consequently, beyond visual lineof-sight (BVLOS) flights are already considered as one flight scenario.

\section{Technical Prerequisites}

Besides general recommendations for pre-flight checks of all technical functionalities, 12 out of 19 countries mention special technical instruments as prerequisites for UAV operations. Here, Chile and Colombia stand out for pervasive and concrete requirements that encompass the material of the blades, the GNSS device, command and control requirements, and the autopilot and recovery capabilities, to name a few. In contrast, the remaining ten countries mainly demand special technical arrangements if the flight operation is classified as risky by either exceeding a certain MTOW and being operated in populated areas. In six cases, the main requirements concern the command and control system and notable failure and safety instruments like parachutes that can safely terminate the flight in emergencies like malfunction or loss of command and control links (e.g., in France and Italy).

In addition to general requirements, collision avoidance capabilities were chosen as a separate variable as it is currently widely discussed for the safe integration of UAVs into national airspace systems ( $Y u$ and Zhang, 2015). In manned aircraft aviation, the pilot observes whether other airspace users are on a collision path and adjusts the aeroplane and flight if necessary. As UAVs are operated without any pilot on board, the 'see and avoid' concept of manned aircraft can no longer be fulfilled. Especially in cases where UAVs are used beyond visual lineof-sight (BVLOS), substitutes like special technical instruments may become necessary to achieve an equivalent level of safety. In this comparative analysis, only seven cases mention sense and avoid, see and avoid or detect and avoid requirements, respectively. However, these mandates are only applicable in particular operational conditions like flights in controlled airspace (UK), BVLOS (France) or UAV operations above a certain MTOW (Canada). In all cases, respective requirements remain very general. The U.S. further outlines the reasoning behind not incorporating any collision avoidance requirements into their regulations as technology is still in its early stage. None of the instruments has an airworthiness certification so far (Federal Aviation Administration, 2016). 


\section{Operational Limitations}

Operational limitations are the main criteria of most UAV regulations and refer to restrictions of the UAV flight. Apart from Nigeria, all countries have defined horizontal distances to people and property, or so-called no-fly zones, which need to be considered. The most prominent example of such a no-fly zone is the surrounding of airports and airstrips. As UAVs pose a severe risk to manned aircraft, they are usually not allowed to fly in controlled airspace and thus in the proximity of places where manned planes land or take off-special authorization might be possible on a case-by-case basis. Besides the prohibition to operate UAVs in controlled airspace, some countries define additional no-fly zones that can be retrieved from online map services (e.g., Japan, the Netherlands). Another essential operational limitation states a safe distance to people, property, and vessels not associated with the UAV flight itself. Here, ten countries specifically mention minimum lateral distances in the range of $30 \mathrm{~m}$ to $150 \mathrm{~m}$ to people. Six countries raise a general prohibition to fly in the vicinity of people and crowds of people. The remaining countries, Nigeria, China and Malaysia, do not discuss operational limitations related to people.

One hierarchal level higher than prohibitions to fly over people refers to flight regulations over congested areas like towns, cities and roads. Here, 12 countries prohibit UAV flights over these areas-some even include a minimum distance needing to be kept. However, terms like congested areas and crowds of people remain vague, and expressions are rarely defined. In contrast, the extent of the restricted regions is very sharp and includes UAV flights over jails, military areas, industrial buildings, nuclear power plants, hospitals, and governmental buildings, respectively. Except for Malaysia and Nigeria, all cases incorporate these kinds of prohibited areas into their legal framework. In addition to permanent restricted areas, emergencies like police or fire brigades operations might be subject to temporal UAV flight restrictions (e.g., Australia, Germany). More exceptional operational limitations refer to a maximum flight time of $60 \mathrm{~min}$ (Chile), a restricted distance of 9.2 $\mathrm{km}$ towards all international borders (Colombia), or a specified distance of $10 \mathrm{~km}$ to any other aircraft (China).

Besides flight restrictions due to the location of the UAV operation, general limitations refer to a maximum height level and horizontal distances in terms of visibility and range. Regarding the height level, all cases apart from China and Nigeria allow only low-altitude flights and define a maximum flying height within the range of a minimum of $90 \mathrm{~m}$ (Canada) to a maximum of $152 \mathrm{~m}$ (Colombia) above ground level. 
These particular heights can be explained by the fact that UAV regulations currently aim to separate manned aircraft and UAVs-and thus allow UAV flights only below the minimum safe altitude for aircraft. Besides international recommendations by ICAO, the value for minimum safety altitude changes in different national contexts, e.g., the U.S., defines non-congested areas as up to 500 feet (152 m) AGL (Federal Aviation Regulations, Sec. 91.119). Regarding the horizontal distance of UAVs to the pilot, regulatory bodies usually distinguish between two ranges: VLOS and BVLOS. All cases of the comparative analysis allow UAV flights in VLOS. In VLOS conditions, the pilot must maintain direct unaided visual contact to the UAV (ICAO, 2015). In addition to this definition, eight countries designate maximum horizontal distances that range from a minimum of $100 \mathrm{~m}$ in France to a maximum of $750 \mathrm{~m}$ in Colombia. The required distance bounds a vague interpretation of the term VLOS. Some cases further include extended visual line-of-sight (EVLOS) operations. Here, the pilot uses an additional observer or remote pilot to keep visual contact with the UAV (cf. Figure 3.5). The US, UK, Italy, Spain and South Africa particularly mention the possibility of EVLOS operations within their UAV regulations. Furthermore, 13 out of 19 countries allow BVLOS flights. However-apart from Spain, France and Nigeria-BVLOS flights are outside the scope of the UAV regulations and thus require either special flight conditions or exceptional approvals.

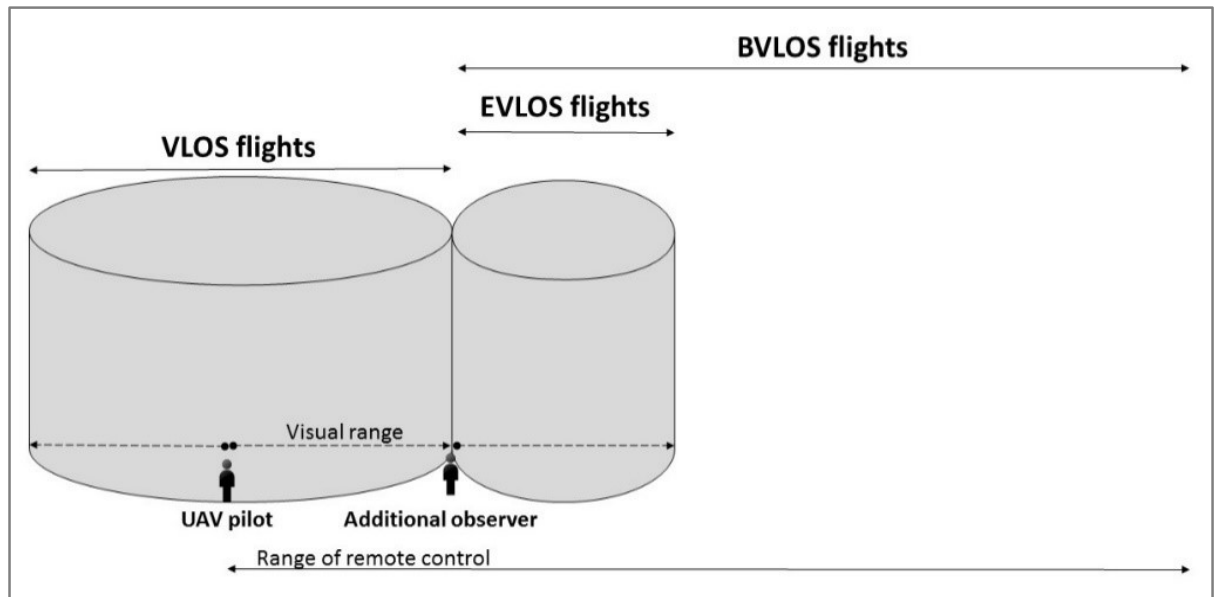

Figure 3.5: Schematic distinction between UAV flight ranges- visual line-ofsight (VLOS), extended visual line-of-sight (EVLOS) and beyond visual line-ofsight (BVLOS), based on (South African Civil Aviation Authority, 2016) 


\section{Administrative Procedures}

The criteria of administrative procedures distinguish variables according to the application process, the need for UAV registration and the need for insurance coverage. Here, marked heterogeneity can be observed. In general, the amount of effort to apply for flight permission depends on the complexity of the UAV operations. Due to the initial classification of different UAV operation scenarios, nearly all application procedures are multi-layered, and various strategies must be followed in various contexts. To provide a comparable basis, the following results are grounded on best-case scenarios where the UAV flight meets all operational prerequisites and does not fall under special approval conditions-if not mentioned otherwise. According to this assumption, huge differences become visible: some countries do not envisage any application procedures for UAV operations below a certain MTOW-neither for the platform's registration nor for a flight approval.

In contrast, others follow a single-case flight authorization approach that entails a new application for each flight operation. Australia, Italy and Canada do not require a formal flight application if the UAV does not exceed an MTOW of $25 \mathrm{~kg}$. Others demand applications if the flight is intended to happen in areas that do not conform to standard operational procedures (Japan, France, UK). Light UAVs in Austria and Germany are subject to general permission, granted for a specified timeframe. In six cases, UAV flight permissions encompass single-case application procedures.

In addition to official and formal flight permission procedures at respective national aviation authorities, notifications and local flight approvals are common praxis for most cases. Based on the regulatory texts, the approval and notification procedure follows four main purposes: (1) to acknowledge specific local operational restrictions; (2) to impede conflicts of airspace users; (3) to avoid concerns and interruptions from the public; and (4) to allow surveillance of the intended UAV flight. Furthermore, declarations of compliance to applicable sections in the UAV regulations can also be mandatory before granting flight permission (e.g., Italy, Spain, UK). If the flight will be (partly) executed in controlled airspace, a notification to the air traffic control service is compulsory in all cases compared. At this, Spain stands out as a particular case. All commercial UAV flights involve a special notification to airmen (NOTAM) regardless of whether the UAV enters controlled or non-controlled airspace.

Besides the notification to other airspace users, announcements to the local police and the approval by the landowner where the UAV is about 
to take-off and land are obligatory (e.g., Germany). At this point, search and rescue operations by fire brigades or UAV operations of governmental institutions are usually exempt from these formal application procedures.

Next to the application procedure, eleven cases require registration for any kind of commercial flight operation. Here, registration involves a registration number, markings, or an electronic ID plate (e.g., Italy). Aviation authorities in Malaysia and Azerbaijan require registration only if the weight of the UAV exceeds $20 \mathrm{~kg}$. Six cases do not embrace any registration process.

Insurance obligations are further considered within the majority of UAV regulations of this analysis. The insurance addresses the subject of a transparent liability regime that can sufficiently compensate for any harm or damage caused by the flight operation (Boucher, 2015). However, in six out of 19 UAV regulations, an adequate insurance policy is not considered compulsory for UAV flights.

\section{Human Resource Requirements}

Besides the UAV itself, many regulations include demands upon the UAV pilot. Practical training, theoretical knowledge tests, aeronautical tests, and medical assessments encompass the most common requirements. Just as with application procedures, the level of required pilot skills usually depends on the flight mission's complexity and risk. So far, Japan does not mention any pilot needs, and Azerbaijan, UK, and Germany only request a basic confirmation of the competencies of the UAV pilot. Besides this, the majority of the cases either demand a pilot certification or a license. The main difference between both is grounded in the amount of training attached to the degree's issuance (cf. Italy). A certificate is usually granted by intermediaries like authorized training centres or UAV manufacturers and entails a pilot's basic practical and theoretical training.

In contrast, the procedure to obtain a UAV pilot license generally involves assessing aeronautical background knowledge, practical examinations and medical tests. National aviation authorities usually issue a pilot license. However, a sharp distinction between pilot qualifications is impossible, and some countries choose "middle ways" and refer either to certificates or licenses. In this comparison, Malaysia and Nigeria are exceptional cases. A UAV operation in Malaysia requires two persons-a pilot and a commander. Both shall hold a valid pilot license. In Nigeria, the UAV pilot needs to be licensed to operate manned aircraft to execute UAV flights. 


\section{Implementation of Ethical Constraints}

The aspect of privacy and data protection concerning the increasing use of UAVs underlines one currently widely discussed topic (Bennett Moses, 2007; van Wegen and Stumpf, 2016). UAVs can be equipped with multiple payloads such as imaging equipment or transmitters that can easily capture and record data of people, houses, or other objects and thus potentially violate citizen privacy and data protection rights. Based on the outcomes of this comparative analysis, these aspects are barely incorporated and reflected in current UAV regulations. Only 12 cases mention privacy and data protection. Here, the majority of cases "only" advise respecting personal privacy. Furthermore, many cases state that actions might be subject to other laws and that national and international applicable legislation must be followed. However, only five countries directly refer to respective laws. Rwanda mainly incorporates the prohibition of surveillance activities without people's consent into their UAV regulations.

\section{Country Context and Pattern}

The comparison of variables of all 19 cases reveals a heterogeneous picture. Even though all countries except Azerbaijan, Chile, Nigeria and Rwanda are part of JARUS, no coherent concept or strategy for national UAV regulations can be identified. Particularly striking examples are Japan and Nigeria, as more than half of the variables were not addressed by their legal frameworks. One would now suggest that both cases are similar. However, the opposite is the case. Without tangible technical and human resource requirements, UAV flights in Japan can easily be commenced within given operational and geographical restrictions. In Nigeria, no general operational limitations or technical requirements are stated.

Nonetheless, each flight needs special authorization, and the pilot requires a manned aircraft license which involves a very elaborate procedure and impedes widespread usage of UAVs. In contrast, France, Italy and Austria acknowledge nearly all variables in their regulations. In all three cases, the regulations show maturity as different scenarios and a complex risk-based classification is recognized. Technical and administrative requirements and operational restrictions are formulated according to the risk of the flight operation. All other cases exclude the area of interest as one classification criterion and primarily refer to special approval procedures if one intends to fly in usually restricted areas-such as developed and inhabited areas. 
Regarding the temporal aspect-date of release or last update-all UAV regulations except Spain and Malaysia were either issued or updated during the previous two years (2015 and 2016). All cases that show updates since the first release tend to involve lower administrative procedures and lower demands for pilot qualification for risk-less UAV operations within respective limitations. During the online search and investigations of the authors, different levels of the provision of information about respective UAV regulations became prevalent. Except for Malaysia, all cases characterized by early releases before 2012 show active public relations activities, dissemination and awareness campaigns of respective regulatory frameworks, including clear homepages that provide insightful infographics and online templates for notification forms. Besides this, a few countries like the US and the Netherlands already embrace different kinds of media and platforms to raise the consciousness of mandatory pre-flight requirements and operational limitations for UAV flights 5 .

\subsection{Discussion}

The following subsections synthesize the outcomes of the research investigation and aim to predict possible future trends. Here, the first part sketches identifiable trends that are based on the comparative analysis of 19 national UAV regulations. In contrast, the second part emphasizes developments in the broader field of law and technology in regard to legal UAV frameworks: the aim is to position current and possible future developments in a broader contextual setting.

\subsubsection{Future Trends and Challenges}

The current state of UAV regulatory frameworks constitutes a significant focus for many stakeholders in the domain (Clothier et al., 2011), and the heterogeneity of national UAV regulations shows varying approaches in response to the demands of various interest groups. However, commonalities are present, and recent changes in some national UAV regulations allow for predicting possible future developments and challenges. For example, civil UAV operations in both controlled and uncontrolled airspace are primarily restricted, impeding broader utilization, at least in the short term.

5 E.g.http://www.veiligvliegen.nl (NL), http://droneaware.org (GB), https://www.faa.gov/uas/where to fly/b4ufly/ (US) accessed on December 2016 
The critical challenge appears to be to find an optimum balance between the demands of the various actors, allowing for innovation on the one hand, but at the same time ensuring recognition and support for safety, fundamental human rights and civil liberties. The future development of civil UAV use will ultimately involve multiple interest groups and various motivations (Clothier, Fulton and Walker, 2008; Rao, Gopi and Maione, 2016). Government institutions and regulatory bodies that hold political mandates want to ensure public safety and security, and civil liberties and promote UAV innovation and technology innovation. Stakeholders in research strive for UAV technical advancement. Hardware and software manufacturers aim to sell products and are interested in lowering market barriers and opening new application areas. End users have their own needs and market interests according to their priorities.

In both national and international contexts, a risk-based approach to regulate the use of UAVs appears to be the strategy of choice. Weight classifications acknowledge an initial step towards risk-based assessments and requirements, which is already evident in most cases. However, including other parameters such as area, purpose, and visibility provides an even more balanced approach. Following this, an all-embracing framework would ultimately streamline regulations for all kinds of civil UAV operations and disentangle complicated requirements for special exemptions and waivers. If a UAV flight can be considered riskless (i.e., lightweight, in uninhabited areas and VLOS) or extremely low risk, no bureaucratic barriers should impede it simply for the sake of bureaucracy. The riskier the flight operation, the more requirements are applicable. This is reflected by the operation category by the current European regulations (cf. Figure 3.4.).

Grounded in this risk-based approach, detailed safety requirements for various scenarios can be inbuilt into the regulatory framework (Clothier et al., 2011). Damage to physical objects or injuries of people on the ground caused by UAVs are threats to public safety. They may result from a technical malfunction or inadequate maintenance of equipment, mid-air collisions, or misuse by its operator. Concerning the growing UAV market and activity levels, the requests for approvals for flights are certain to increase. In this context, aviation authorities would presumably prefer to avoid treating every request as a stand-alone request: the administrative time and expense would overwhelm bureaucracies and undermine any policies intended to promote technological innovation. Increased efficiency and capacities to deal with the administrative processes of flight approvals and UAV platform registration are necessary. In addition, general flight permissions for 
low-risk UAV operations in uncontrolled airspaces, such as in large parts of Australia and Canada, can be seen as pioneering. However, additional (in the best case, online) notification forms-with details of the date, time, and place of the intended UAV operation-are essential to allow the safe and efficient management of respective airspace and help avoid mid-air UAV collisions. In this regard, it is evident that countries with a long history of frequently updated UAV regulations show more maturity than countries that first implemented regulations only in $2015 / 16$. Here, maturity can lead to efficient administrative processes, awareness campaigns, and established procedures to register UAVs and to train UAV operators. Next to the more obvious causes of failure of UAV operations, unencrypted signals leave UAVs open to cyber-attacks and hacking. Here, manufacturers are expected to take action to increase the security aspects of their UAVs. Besides others, encrypted signals of the command and control links can impede unintentional interferences widely (ICAO, 2015).

Besides administrative procedures, accountability addresses another key aspect when it comes to UAVs and public safety. The operator's responsibility lasts for the entire flight mission and thus involves being aware of and adhering to legal regulations from the beginning of the UAV mission. However, UAV regulations can hardly be found in print, let alone on the homepages of aviation authorities. This situation was found in some countries where few cases were present in information services and consulting initiatives. Given the political will to foster the use of civil UAVs, educational modules, easily accessible information services and awareness campaigns that explain prevailing legal norms need to be developed, promoted, and made readily accessible. Lessons learned, best practices, and the awareness of consequences of misuse can reassure the public, thereby creating trust. As well as accountability in general, auditing the platform and pilot is necessary to investigate what happened in case of an accident and hold the responsible person accountable. This can only be achieved if the UAV platform carries unique identifiers like registration numbers or special ID plates. Although platform registration schemes exist in many countries, this requirement is also likely to become mandatory for the remaining countries. Once the UAV-and consequently also the pilotcan be identified after an incident that causes injury to people or damage to property, sufficient insurance coverage must be available to compensate for injury or damage. Accordingly, the financial risk of operating UAVs is transferred from the operator to the insurer. However, insurance companies impose their conditions relating to UAV operations and thus can set more requirements than mandated by 
national legal frameworks. As liability refers to one main concern, mandates for apportioned insurance coverage and, therefore, the influence of insurance companies in regulating UAV operations are expected to grow. Besides the definitive liability of the use of UAVs, liability for design and construction of UAVs are foreseeable aspects for future debates as a legal basis still has to be defined. A clear divide of responsibilities amongst those involved becomes particularly necessary when the degree of automation of UAV flights increases (European RPAS Steering Group, 2013).

Concerning the growing number of UAV operations, onboard communication devices can play a vital role for BVLOS-and even beyond radio line-of-sight flights-to avoid mid-air collisions. Nokia is an example of a firm currently developing technology for UAV-based traffic management. It is conceivable that UAVs in non-segregated airspace need to be equipped with communication devices that allow for safe operations with other airspace users-both manned and unmanned aircraft. Central flight coordination services must know where, when and which UAV is flying at each point in time to monitor and safely manage all operations. In this regard, the law will likely respond to such technological developments and set requirements for onboard devices. Accordingly, it seems most likely that UAVs that are not equipped with special communication techniques will only be allowed to fly in segregated airspace, i.e., in UAV test centres or special zones for private users who wish to use UAVs for recreational purposes.

The ability of UAVs to collect information on individuals and private places may infringe the right to privacy and question the protection of personal data. Following the outline of relevant publications (Finn and Wright, 2014; Jansen, 2015; Marzocchi, 2015; Rao, Gopi and Maione, 2016) and supported by the results of this comparative analysis, the challenge of data protection and the right to privacy is hardly being addressed in any of the national and international UAV regulations so far. However, a broad scientific discourse has already been initiated, and this topic continues to gain importance. Although national and international laws and regulations are mostly in place and implicitly deal with these ethical concerns, two main problems remain:

(1) gaps in the respective laws and regulations governing the use of UAVs (European RPAS Steering Group, 2013; Marzocchi, 2015),

(2) the lack of awareness about data protection and privacy rules (Finn and Wright, 2014; Marzocchi, 2015). 
The characteristics of UAVs-being both low cost in operation and small in size-make it very difficult to control and track them to ensure lawful use and hinder targeted observations and surveillance by individuals. Moreover, data collection by UAVs remains invisible in most cases, and the data subject is rarely aware that their data is being collected (Finn and Wright, 2012). Without knowledge about the operator, purpose of the flight and sensing equipment the UAV is equipped with, individuals face significant challenges enforcing their rights (Finn and Wright, 2014). All possible threats to the right to privacy and the use of personal data captured by UAVs should be assessed to incorporate them into national privacy and data protection laws adequately. Furthermore, following the outline of (European RPAS Steering Group, 2013), distinct boundaries for UAV operators and strict limitations regarding the ownership and further storage and dissemination of collected data need to be addressed in respective regulatory frameworks. To fully comply with legal demands, awareness-raising actions and communications between industry, users, and the general public need to be stimulated. In addition to this, easily accessible information platforms and soft law measures that are subject to guides, code of conduct and impact assessments on privacy are essential tools to adequately address challenges to fundamental rights regarding the utilization of UAVs. Privacy by design (Cavoukian, 2012; Marzocchi, 2015) might also be a future option and refers to design-specific technically embedded data protection measures.

International organizations are devoting much effort to formulate common standards such as prescriptive requirements for UAV operations (ICAO, 2011), technical standards for UAVs (JARUS, 2013), and pilot licensing recommendations (JARUS, 2015). Examples include the JARUS standards of light rotorcraft UAVs or the evolution of VLOS and BVLOS flights as defined in the European roadmap (European RPAS Steering Group, 2013). According to the latter, VLOS operation over populated areas should have already reached the ultimate goal of successfully integrating non-segregated airspace. However, only a few countries envisage UAV operations in populated areas without special permission. The same applies to BVLOS flights, which are rarely mentioned in national UAV regulations, but should by now have reached a certain level of national/international integration. This is mainly attributed to technological causes because an equivalent level of collision avoidance capabilities is yet to be developed. Besides the European context, global recommendations, such as those from ICAO and JARUS, are expected to gain relevance. They play a crucial role in 
paving the way for the convergence of national regulations towards international harmonized standards.

\subsubsection{Unmanned Aerial Vehicles (UAVs) in the Broader Context of Law and Technology}

Apart from foreseeable future developments of national and international UAV regulations, "history has shown us that some of the greatest obstacles facing the realisation of a new technology are not always technical in nature but are often related to its integration into society" (Clothier, Fulton and Walker, 2008). The same authors reviewed the developments of the regulations of early automobiles in the UK and presented insights about the risk management of new technologies in general. In this context, resentments, conspicuous drawbacks, limited public knowledge about the technology and threatened industrial competitors paved the way for the very precautionary formal regulations of automobiles in the UK (cf. "Red Flag Law" 1865). For 30 years, this law blocked further technological developments in the UK, leaving other countries to benefit from pioneering. In 1930, a comprehensive law included regulations on construction, weight, driver licensing, insurance obligations, and penalties defined. Although some differences exist, (Clothier, Fulton and Walker, 2008) show clear parallels with current UAV regulations, allowing for hypotheses about future regulatory developments in a broader context.

Existing proscriptive and prescriptive legal frameworks for UAVs are expected to change in the future. However, due to the ongoing emergence of new UAV technology, slowly adapting UAV regulations are limited in keeping the link to current developments (Bennett Moses, 2013). The main problems concern the constant need to address further potential harms, risks and negative impacts (Marchant, Allenby and Herkert, 2011). Therefore, soft laws as alternative means of rulemaking may also play a critical role in adequately addressing the gaps left by formal regulation. One commonly distinguishes between co-regulation, industry self-regulation and organisational selfregulation, resulting from the varying influences of the state, industry associations, corporations, and other stakeholders (Clarke and Bennett Moses, 2014). In the context of a review, the same authors found only a small number of initiatives in all three areas. However, concerning the growing UAV industry and the resulting competition, it is expected that efforts towards industrial co-regulation will gain importance. Key players will likely play an essential role in establishing internationally recognized standards to increase entry barriers against new market 
competitors. Industrial design standards for UAV components or standardized communication devices that prevent mid-air collisions are conceivable examples. Besides the influence of industrial manufacturers, professional users are expected to play another key role in regulating the use of UAVs. Their involvement will probably contribute to the decision as to whether UAVs will be a tool for everyone or just for professionals. According to the market interests of professional UAV users, their vote is likely to be for the benefit of already established UAV professionals and conventional image suppliers who extended their business by taking stakes in UAV companies.

As already discussed earlier, public acceptance is one cornerstone for the integration of UAVs into society. Here, public awareness and familiarity with the UAV technology and evident societal benefits will probably push acceptability. Current media coverage is mostly very polarized, shaping citizens' sentiments towards both positive and negative extremes. However, existing resentments can be neutralized by educational processes, awareness campaigns and readily accessible and authoritative information. Furthermore, it is essential to highlight the nature of public acceptance: this mainly refers to accepting the associated risks rather than the technology itself (European RPAS Steering Group, 2013). The same report further pointed out that the public shows a tendency to "overestimate small risks and to underestimate large risks." Thus, information about best practices and the awareness of potential benefits to the society will increase existing knowledge about UAV technology and help developing objective opinions about associated risks: "[This] will be influential in shaping public perceptions, and ultimately, acceptance of [UAV] technologies" (Clothier et al., 2015).

In essence, besides using law as a tool to manage risk, (Ison, Röling and Watson, 2007) identified market mechanisms and information provision as other ways of controlling behaviour. Although that particular framework was published in the context of the sustainable management and use of water, parallels to how to regulate UAVs can be drawn. As outlined in the results and discussion section, past, present, and future trends can be linked with developments in the law, the market, or information (Figure 3.6). Currently, legal frameworks are the driving force that regulates the use of UAVs. However, due to the growing UAV market, the market mechanism will soon gain the most importance. Furthermore, information and education as enabling tools for public acceptance and broad integration of UAVs into society are expected to receive increasing attention. However, without the 
basic requirement of political will, changes are unlikely to happen. Although UAV regulations are in place in more than half of all countries (as of October 2019), gaps and lack of capacity can be seen in enforcement and implementation.

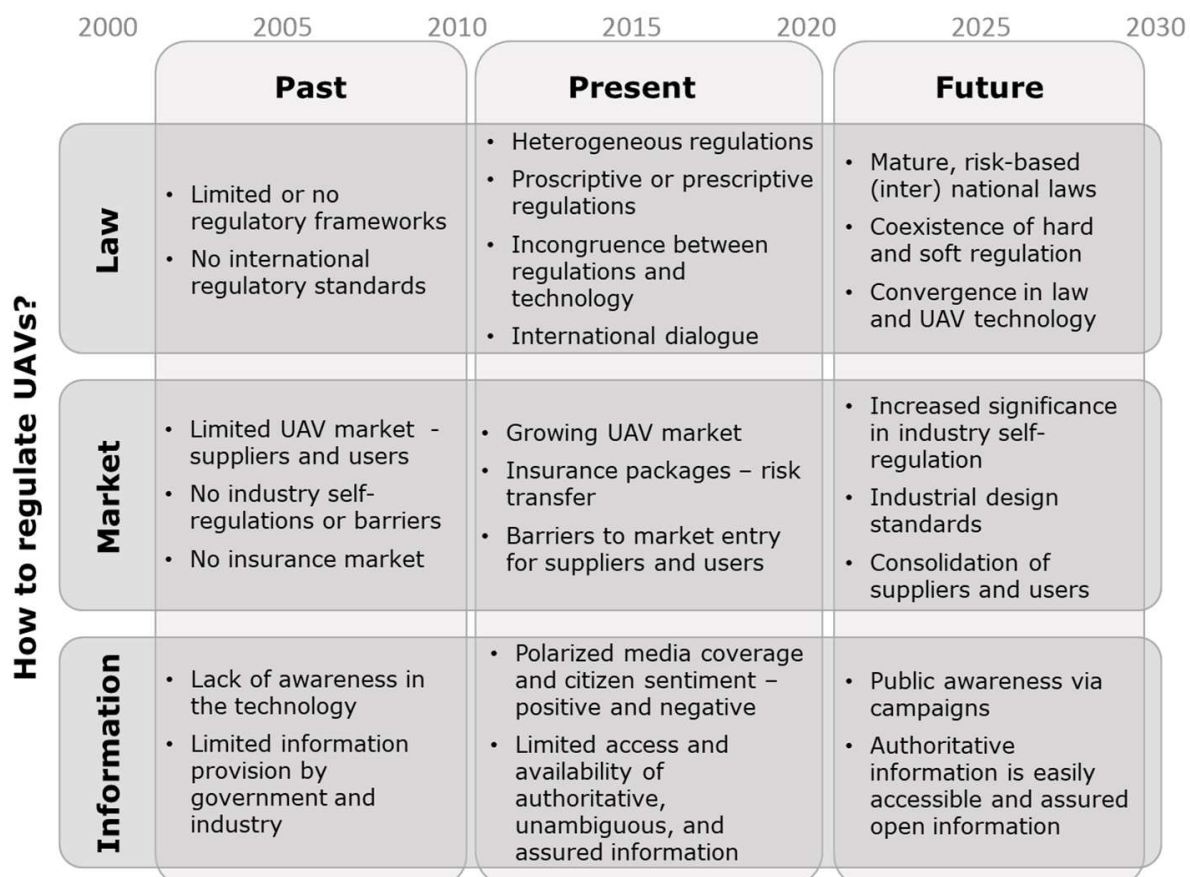

Figure 3.6: Consolidated overview of past, present and future developments distinguished according to main regulatory mechanism law, market and information.

\subsubsection{Limitations of This Research}

Regarding the extent of this research, the number of variables was limited to the insights that could be gained by reviewing national regulatory frameworks that deal with UAVs. However, additional criteria, such as political will, the establishment of dedicated institutional units, rulemaking parties, and social acceptance, could also be valuable sources of information but would demand other research methods that were not within the scope of this chapter. Although enacted UAV regulations are expressed in tangible documents, they do not allow insights "behind the scenes" such as political backgrounds, law enforcement, human capacities and processes. Thus, the presence of UAV regulations does not necessarily mean that UAV operations will be straightforward. 


\subsection{Conclusions}

This research investigation is the first to provide a holistic global overview of the current status of unmanned aerial vehicle (UAV) regulations. It further delivers insights into the past, present and future development of legal frameworks that regulate the use of UAVs. Based on a research synthesis that includes a thorough literature review and comparative analysis of 19 national regulatory frameworks and their status in 2016, similarities and contrasting elements in various national UAV regulations and their implications for data acquisition activities are explored. In essence, regulations target the management of risks and minimization of perceived harms. Within the context of UAVs, the main harms are malfunction, mid-air collisions and consequential damages to people and property on the ground. To address these harms, it is found that UAV regulations focus upon three key aspects: (1) targeting the regulated use of airspace by UAVs; (2) imposing operational limitations; and (3) tackling the administrative procedures of flight permissions, pilot licenses and data collection authorization. Since the early 2000s, countries have gradually established national legal frameworks for UAVs. Although all UAV regulations aim at one common goal, minimizing the risks for other airspace users, people, and property on the ground, a distinct heterogeneity of national regulations exists. However, commonalities such as mandatory platform registration, obligatory insurance coverage and standard pilot licensing procedures indicate trends towards mature national or even international UAV regulations, as seen by the efforts of the European Union. Desirable trends are seen in the coexistence of hard and soft regulations and the successful international dialogue that will eventually provide a legal framework for harmonized regulatory standards. Besides the strong presence of law, market forces such as industry design standards and reliable information are public goods that are expected to shape future developments.

One direction for further research is related to the implementation of UAV regulations. Although this review focuses mainly on the content of respective regulatory frameworks, it can only provide limited statements regarding internal administrative processes, local capacities, and subsequent implications for disseminating UAV technology. Another direction for further research entails a detailed investigation of the impact of the release of UAV regulations on realized UAV flights and economic developments in UAV businesses. 


\section{Chapter 4-Guidance for Optimal UAV Flight Configurations to Derive High-Quality Orthophotos $^{6}$}

6 This chapter is based on:

Stöcker C, Nex F, Koeva M, Gerke M. High-Quality UAV-Based Orthophotos for Cadastral Mapping: Guidance for Optimal Flight Configurations. Remote Sensing. 2020; 12(21):3625. https://doi.org/10.3390/rs12213625 


\subsection{Introduction}

The fact that UAV-derived geospatial information can support decisionmaking processes involving people's land rights raises questions about the quality of UAV data. In this context, the concept of quality is closely linked to spatial accuracy, which can be defined as absolute (external) or relative (internal) accuracy. According to (ISO, 2013), absolute accuracy refers to the closeness of reported coordinate values to values accepted as or being true. In contrast, relative accuracy describes the similarity of relative positions of features in the scope to their respective relative positions accepted as or being true. Both measures are equally crucial in land administration contexts (c.f. Grant et al., 2020); firstly, the correct representation of image objects such as houses or walls (relative accuracy) as well as the correct position of corner points (absolute accuracy) (ISO, 2013). Generally speaking, the spatial accuracy depends on the UAV flight mission configurations such as sensor specifications, UAV itself, mode of georeferencing, flight pattern, flight height, photogrammetric processing, image overlap, and external factors such as weather, illumination, or terrain.

During the past decades, remote sensing and computer vision communities alike studied those impacting parameters emphasizing image matching algorithms, different means of georeferencing, and various flight planning parameters, among others. Finding accurate and reliable image correspondences is the basis for a successful imagebased 3D reconstruction. Numerous authors investigated this fundamental part of the photogrammetric pipeline while increasing the precision of image correspondences and optimising computational costs (Förstner and Gülch, 1987; Lowe, 2004; Snavely et al., 2006; Remondino et al., 2014). The quantity of tie-points derived during feature matching mainly depends on the type and the content of the image signal. Deficient success rates negatively impact the spatial accuracy and overall reliability of the 3D reconstruction and ultimately worsen the quality of the DSM and orthophoto (Gruen, 2012).

Next to the aspect of feature matching, georeferencing refers to one of the most practice-relevant yet most discussed topics when utilizing UAV imagery for surveying and mapping applications. More than 60 studies examined various methods of sensor orientation for terrestrial applications, as outlined by (Singh and Frazier, 2018). The choice for a georeferencing approach typically represents trade-offs between spatial accuracy and operational efficiency (Rehak and Skaloud, 2016). Even though direct sensor orientation or integrated sensor orientation brings significant time-savings for the data collection operation, 
planimetric accuracies usually range between 0.5 to $1 \mathrm{~m}$ due to the low accuracy and reliability of directly measured attitude and positional parameters by onboard navigational units without a reference station (Haala et al., 2012; Pfeifer, Glira and Briese, 2012; Turner, Lucieer and Wallace, 2014). Note that because of inaccurate scale estimation coming along with those insufficient methods, the absolute and relative accuracy might not be suitable for a particular application. With the use of real-time kinematic (RTK) or post-processing kinematic (PPK) enabled GNSS devices, spatial accuracies can be improved to $\mathrm{cm}$-level of accuracy (Eling, Klingbeil and Kuhlmann, 2014; Gerke and Przybilla, 2016; Hugenholtz et al., 2016; Stöcker, Nex, et al., 2017; Forlani et al., 2018). However, issues of sensor synchronization, as well as insufficient lever-arm and boresight calibration, remain challenging (Stöcker, Nex, et al., 2017; Ekaso, Nex and Kerle, 2020), particularly for off-the-shelf UAVs.

In addition to positional or full aerial control, integrated sensor orientation offers the option to include ground observations, known as ground control points (GCPs). This has proven beneficial to mitigate systematic lateral and vertical deformations in the resulting data products (James et al., 2014). Various studies addressed the impact of the survey design of GCPs in terms of quantity and distribution. In their meta-study, (Singh and Frazier, 2018) did not find a clear relationship between the number of GCPs and the size of the study area but investigated a weak negative relationship between statistics of the residuals and the number of GCPs collected per hectare. Data from several sources confirm that the distribution of GCPs strongly impacts spatial accuracy, and an equal distribution is recommended (MesasCarrascosa et al., 2015; Manfreda et al., 2019; Villanueva and Blanco, 2019). However, looking at the results of the optimal number of GCPs, different conclusions are evident. Results from relatively small study sites suggest that the vertical error stabilizes after 5 or 6 GCPs (Tonkin and Midgley, 2016; Manfreda et al., 2019) and the horizontal error after 5 GCPs (Mesas-Carrascosa et al., 2015; Manfreda et al., 2019). In contrast, (Sanz-Ablanedo et al., 2018) obtained a low spatial quality with $5 \mathrm{GCP}$ and recommended using a medium to a high number of GCPs to reconstruct large image blocks accurately. Other studies (Agüera-Vega, Carvajal-Ramírez and Martínez-Carricondo, 2017; Oniga et al., 2020) achieved similar results with a concluding recommendation to integrate 15 or $20 \mathrm{GCPs}$ in the image processing workflow, respectively.

Aside from GCPs, higher spatial accuracy can be achieved by additionally including oblique imagery (James et al., 2017) or 
perpendicular flight strips (Gerke and Przybilla, 2016). In most cases, checkpoint residuals were measured in the point cloud or obtained directly after the bundle block adjustment. Thus, they do not necessarily represent the displacement of image points in the final data product, as potential offsets during the orthophoto generation were not considered. However, particularly for the application in cadastral mapping, the correct estimation of spatial accuracy is vital.

Even though weak dependencies between several impacting factors on the data quality are evident, the results of existing studies are very heterogeneous. Furthermore, most studies remain narrow in focus, dealing mainly with only one study site situated in non-populated areas, and it is questionable whether recommendations can be transferred to the cadastral context. To the best of the authors' knowledge, existing studies on UAV-based cadastral mapping only highlight the usability of UAVs without assessing different flight configurations or the impact on the final absolute or relative accuracy. To this end, a comprehensive analysis of varying data quality measures should provide a factual basis for clear recommendations that ensure data quality for UAV-based cadastral mapping. Thus, this chapter seeks to conclude on best practice guidance for optimal flight configurations by integrating results of a detailed quality assessment including three main aspects: (1) feature matching, (2) ground-truthing, and (3) reconstruction of cadastral features. Whereas the first two approaches target the data quality evaluation during and after photogrammetric processing, the latter method focuses on the implications of different orthophoto qualities for the automated extraction of cadastral features. Similar to diverse quality assessment practices, research data are also manifold and drawn from six study sites in Africa and Europe.

In many low- and middle-income countries, conditions for flying, controlling and referencing respective data are more complex than in western-oriented countries, a situation which is often underestimated. Especially spatial and radiometric accuracy can be negatively influenced by poor flight planning and adverse meteorological conditions. Moreover, ground control measurements can be problematic due to a lack of reference stations, the availability of professional surveying equipment, or capacity. In the field of land administration in general and cadastral mapping in particular, incorrect geometries of the orthophoto might cause negative consequences to civil society as the subject deals with a spatial representation of land parcels and attached rights and responsibilities. For example, 
erroneous localization and estimations of parcel sizes might imply inadequate tax charges, problems with land compensation funds, or challenges to merge existing databases spatially. With its unique combination of methods and integration of various study sites, it is hoped that the results and recommendations presented here help land administration professionals and bottom-up initiatives alike to optimise existing and future data collection workflows.

The remainder of the chapter is structured as follows. Section two provides background information on data collection, data processing and quality assessment methods. The results section is divided into three separate subsections with 1) findings showing the impact of land use on the number of tie-points, 2) a comprehensive comparison of different ground control setups and their effect on the final absolute accuracy, and 3) an evaluation of qualitative and quantitative characteristics of extracted cadastral features. The discussion critically reflects on the results based on existing literature and outlines best practice guidance for UAV-based data collection workflows in land administration contexts.

\subsection{Material and methods}

The study setup foresaw three different means of quality assessment targeting absolute and relative accuracy as outlined in the conceptual framework in Figure 4.1. Well-known methods as the statistical evaluation of checkpoint residuals were combined with quantitative measures of image matching results and characteristics of automatically delineated cadastral features. Different clues on spatial accuracy substantiate the results to provide best practice guidance. Detailed workflows and specifications of the analysis are outlined below. 


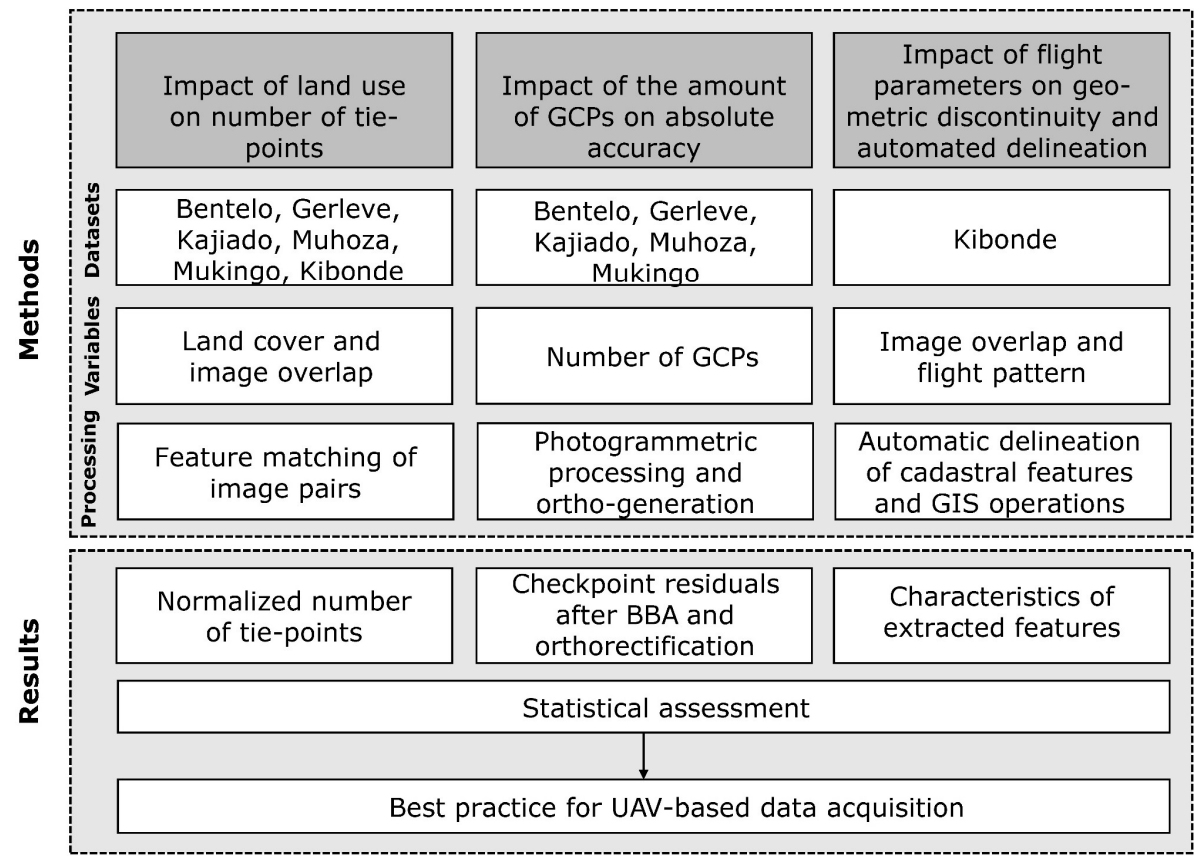

Figure 4.1: Conceptual framework of chapter 4

\subsubsection{UAV and GNSS data collection}

To test the transferability of the findings and ultimately claim bestpractice recommendations, methods were applied to different datasets collected with diverse UAVs and sensor equipment. This includes, in total, six study areas across Europe (Gerleve, Bentelo) and Africa (Kajiado, Kibonde, Muhoza, Mukingo) ranging from $0.14 \mathrm{~km}^{2}$ to $8.7 \mathrm{~km}^{2}$ (Figure 4.2). The UAV equipment, as well as sensor specifications, are outlined in Table 4.1 and include two fixed-wing UAVs (Ebee Plus, DT18), one hybrid UAV (FireFly6), and two rotarywing UAV (DJI Inspire 2, DJI Phantom 4) equipped with an RGB sensor. Two out of the five UAVs worked with a PPK. Prices for the platforms and sensors range from 1.000 to 40.000 EUR. Flights in Gerleve, Bentelo, Muhoza, Kajiado, and Mukingo were carried out according to a classical flight pattern without cross-flights and an overlap of $80 \%$ forward overlap and $70 \%$ side lap for all datasets. Additionally, the study in Kibonde foresaw several flights that were repeatedly carried out with varying image overlap ( $60 \%, 70 \%$, and $80 \%$ side lap) to assess the impact of flight parameters on the characteristics of extracted cadastral features. Following existing literature that proves the benefit of cross flight patterns (Gerke and Przybilla, 2016), three perpendicular strips in a different flight height were added to the 
regular flight and are part of the accuracy evaluation for the dataset Kibonde as well.

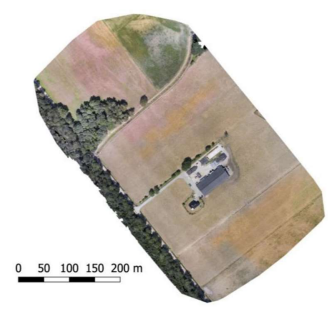

a) Bentelo (The Netherlands)

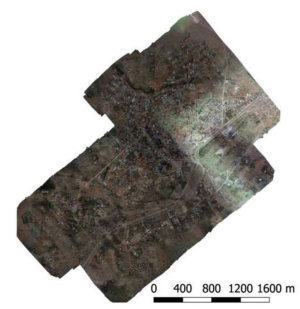

d) Kajiado (Kenya)

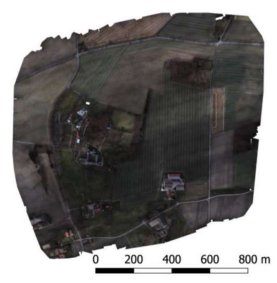

b) Gerleve (Germany)

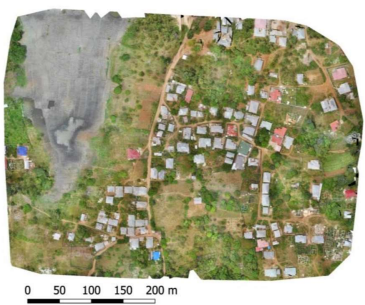

e) Kibonde (Tanzania)

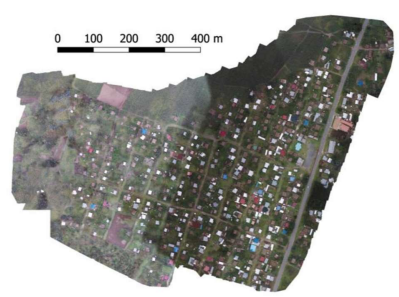

c) Mukingo (Rwanda)

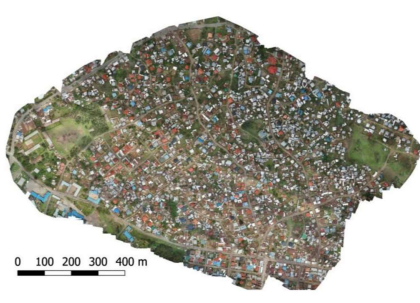

f) Muhoza (Rwanda)

Figure 4.2: Overview of all UAV datasets presented as orthomosaics a) Bentelo, b) Gerleve, c) Mukingo, d) Kajiado, e) Kibonde, and f) Muhoza (scales vary).

Table 4.1: UAV equipment and technical specifications of the sensor

\begin{tabular}{|c|c|c|c|c|c|}
\hline Dataset & $\begin{array}{l}\text { Area } \\
{\left[\mathrm{km}^{2}\right]}\end{array}$ & $\begin{array}{l}\text { GSD } \\
{[\mathrm{cm}]}\end{array}$ & UAV & Camera & $\begin{array}{l}\text { Sensor size } \\
{[\mathrm{mm}]}\end{array}$ \\
\hline Muhoza & 0.98 & 2.1 & $\begin{array}{l}\text { BirdEyeView } \\
\text { FireFLY6 }\end{array}$ & $\begin{array}{l}\text { SONY } \\
\text { ILCE- } \\
6000\end{array}$ & $13.50 \times 15.60$ \\
\hline Mukingo & 0.50 & 2.2 & $\begin{array}{l}\text { DJI Inspire } \\
2\end{array}$ & $\begin{array}{l}\text { DJI } \\
\text { FC652 }\end{array}$ & $13.00 \times 17.30$ \\
\hline Kajiado & 8.70 & 5.8 & $\begin{array}{l}\text { DJI } \\
\text { Phantom } 4\end{array}$ & $\begin{array}{l}\text { DJI } \\
\text { FC330 }\end{array}$ & $06.20 \times 04.65$ \\
\hline Kibonde & 0.15 & 3.0 & $\begin{array}{l}\text { SenseFly } \\
\text { Ebee Plus }\end{array}$ & $\begin{array}{l}\text { SenseFly } \\
\text { S.O.D.A. }\end{array}$ & $12.70 \times 08.50$ \\
\hline Gerleve & 1.10 & 2.8 & $\begin{array}{l}\text { DelairTech } \\
\text { DT18 }\end{array}$ & $\begin{array}{l}\text { DT } \\
\text { 3Bands }\end{array}$ & $08.45 \times 07.07$ \\
\hline Bentelo & 0.14 & 2.7 & $\begin{array}{l}\text { DJI } \\
\text { Phantom } 4\end{array}$ & $\begin{array}{l}\text { DJI } \\
\text { FC330 }\end{array}$ & $06.20 \times 04.65$ \\
\hline
\end{tabular}

To allow the inclusion of external reference points into the bundle block adjustment (BBA) and for means of independent quality assessment, 
GCPs were deployed. Due to different contexts and time delays between marking and the data collection flights, different shapes and methods to mark control points were used. In Musanze, Mukingo, Bentelo, and Kibonde quadratic plastic tiles with two equally sized black and white squares were fixed with iron pegs. Crosses marked with permanent white paint were used in Kajiado, as the flight missions took several days. For Gerleve, white sprayed Compact Disks were deployed and fixed with survey pins. Three-dimensional coordinates of the central point were determined with survey-grade GNSS devices. As Continuous Operating Reference Stations (CORS) are only available at a few locations in Africa, different modes were used to achieve a measurement accuracy of less than $2 \mathrm{~cm}$. Real-time CORS corrections could be harnessed in Europe, while a base-rover setting over a known survey point and radio-transmitted real-time corrections or a classical post-processing approach was the preferred surveying operation for the African missions. All GCPs were measured twice, before and after the UAV flight. The average of both measurements was converted from the local geodetic datum to WGS84 or ETRF89. A detailed list of specifications about the GNSS device, the number of measured control points, and original and target geodetic datums are given in Table 4.2.

Table 4.2: Specifications of GCP measurements

\begin{tabular}{|c|c|c|c|c|}
\hline Dataset & $\begin{array}{l}\text { GNSS } \\
\text { device }\end{array}$ & $\begin{array}{l}\text { Measured } \\
\text { points }\end{array}$ & $\begin{array}{l}\text { Original } \\
\text { datum }\end{array}$ & $\begin{array}{l}\text { Target } \\
\text { datum }\end{array}$ \\
\hline Muhoza & Leica CS10 & 17 & ITRF 2005 & $\begin{array}{l}\text { WGS84 } \\
\text { UTM35S }\end{array}$ \\
\hline Mukingo & Leica CS10 & 19 & ITRF 2005 & $\begin{array}{l}\text { WGS84 } \\
\text { UTM35S }\end{array}$ \\
\hline Kajiado & $\mathrm{CHC} \times 900+$ & 16 & Cassini & $\begin{array}{l}\text { WGS84 } \\
\text { UTM37S }\end{array}$ \\
\hline Kibonde & $\begin{array}{l}\text { Sokkia } \\
\text { Stratus }\end{array}$ & 11 & Arc1960 & $\begin{array}{l}\text { WGS84 } \\
\text { UTM37S }\end{array}$ \\
\hline Gerleve & Trimble & 22 & ECEF & $\begin{array}{l}\text { ETRS89 } \\
\text { UTM32N }\end{array}$ \\
\hline Bentelo & Leica GS14 & 18 & Amersfoort & $\begin{array}{l}\text { WGS84 } \\
\text { UTM32N }\end{array}$ \\
\hline
\end{tabular}

\subsubsection{Estimating the impact of land cover on the number of automated tie points}

The establishment of image correspondences is a crucial component of image orientation. In the first step, primitives are extracted and defined by a unique description. Secondly, the descriptors of 
overlapping pictures are compared and correspondences determined. With a low number of automated tie-points, the image orientation is less reliable and negatively impacts the quality of subsequent image matching processes. Different land use classes were defined (cf. Table 4.3 ) to evaluate the impact of land cover on the number of automated tie-points. If a particular land use was present in a dataset, representative image pairs were manually selected and processed as described below.

Table 4.3: Land use classes and representation in datasets (Bentelo, Gerleve, Kajiado, Kibonde, Muhoza, Mukingo). Digits indicate the number of image pairs used for the experiment. Percentage, as outlined in the definition, refers to pixels representing specific land cover.

\begin{tabular}{|c|c|c|c|c|c|c|c|}
\hline Land Use Class & Definition & B & G & $\mathbf{K a}$ & $\mathbf{K i}$ & & Muk \\
\hline Forest & $>70 \%$ covered by trees & 4 & 5 & & & & \\
\hline $\begin{array}{l}\text { Agriculture } \\
\text { (cropland) }\end{array}$ & $\begin{array}{l}>70 \% \text { cultivated } \\
\text { agricultural fields }\end{array}$ & 5 & & & & & 5 \\
\hline $\begin{array}{l}\text { Agriculture } \\
\text { (Grassland or } \\
\text { uncovered soil) }\end{array}$ & $\begin{array}{l}>70 \% \text { bare soil or } \\
\text { sparse grass vegetation }\end{array}$ & 5 & 5 & 5 & 5 & & 5 \\
\hline Rural context & $\begin{array}{l}<20 \% \text { structures, a } \\
\text { predominance of } \\
\text { agricultural activities }\end{array}$ & & & 5 & 5 & & 5 \\
\hline Peri-urban context & $20-70 \%$ structures & & & 5 & 5 & 5 & 5 \\
\hline Urban context & $\begin{array}{l}>70 \% \text { structures, } \\
\text { densely populated }\end{array}$ & & & 5 & & 5 & \\
\hline
\end{tabular}

Most commercial photogrammetric software packages do not provide information on their image matching techniques, and respective codes might be subject to frequent changes. Instead of using such a blackbox software, we chose three state-of-the-art feature matching approaches which were selected, reflecting the variety of blob and corner detectors with binary and string descriptors: SIFT (Lowe, 1999), SURF (Bay et al., 2008), and AKAZE (Alcantarilla, Nuevo and Bartoli, 2013). The open-source photogrammetric software PhotoMatch (Gonzales-Aguilera et al., 2020) was utilized to carry out the tests. Before the feature matching process, all images were pre-processed by a contrast-preserving decolourization tool (Lu, Xu and Jia, 2012), maintaining the full image resolution. The feature matching was conducted with a Brute-Force method and supported by RANSAC for filtering wrong matches. Thus, image correspondences are searched by comparing each key point with all key points in the overlapping 
image. Settings for feature extraction and description were kept to default values as this analysis is meant to detect relative changes of feature matching rates according to the type of land cover instead of performance evaluation of different approaches. The resulting tiepoints (i.e. inlier of key-point matches) were normalized according to the image resolution to reach comparability between different sensor specifications within one land use class. To enable an evaluation of matching quality and variation in different land use classes and feature extraction/matching technique, the number of matches per image pair gets normalized concerning the number of matches within a certain matching algorithm; see equation below for the so-called z-score. Here $A T P$ indicates the normalized number of automated tie-points per image pair (ATP), $\overline{A T P}$ the mean of all matches of the respective feature extraction approach, and $\sigma$ ATP the standard deviation of all matches of the respective matching approach. The z-score provides insights on how many standard deviations below or above the mean the quantity of tie-points in comparison to the other algorithms, within a land use class, is.

$$
Z \text { score }=\frac{(A T P-\overline{A T P})}{\sigma A T P}
$$

To visualize absolute quantities, the mean value of ATP for all different datasets in the same land use class was calculated. Furthermore, the overlap of image pairs was added as an additional variable. For this analysis, data from Kibonde and Bentelo served as input image pairs as both datasets offered various overlap configurations.

\subsubsection{Estimating the impact of the number of GCPs on the final geometric accuracy}

All images were processed using Pix4D, keeping the original image resolution. Point clouds were created with an optimal point density, and digital surface models (DSM) and the orthomosaics were produced with a resolution of 1 ground sampling distance (GSD). To allow the comparability of the spatial accuracy of different datasets, uniformly distributed GCPs were included in the processing pipeline according to a standard pattern (Figure 4.3). Ground markers were identified and linked to at least six images. Depending on the specific number of GCPs $(0-10)$, the remaining points were used as independent checkpoints to estimate the vertical and horizontal accuracy of the final data products. 


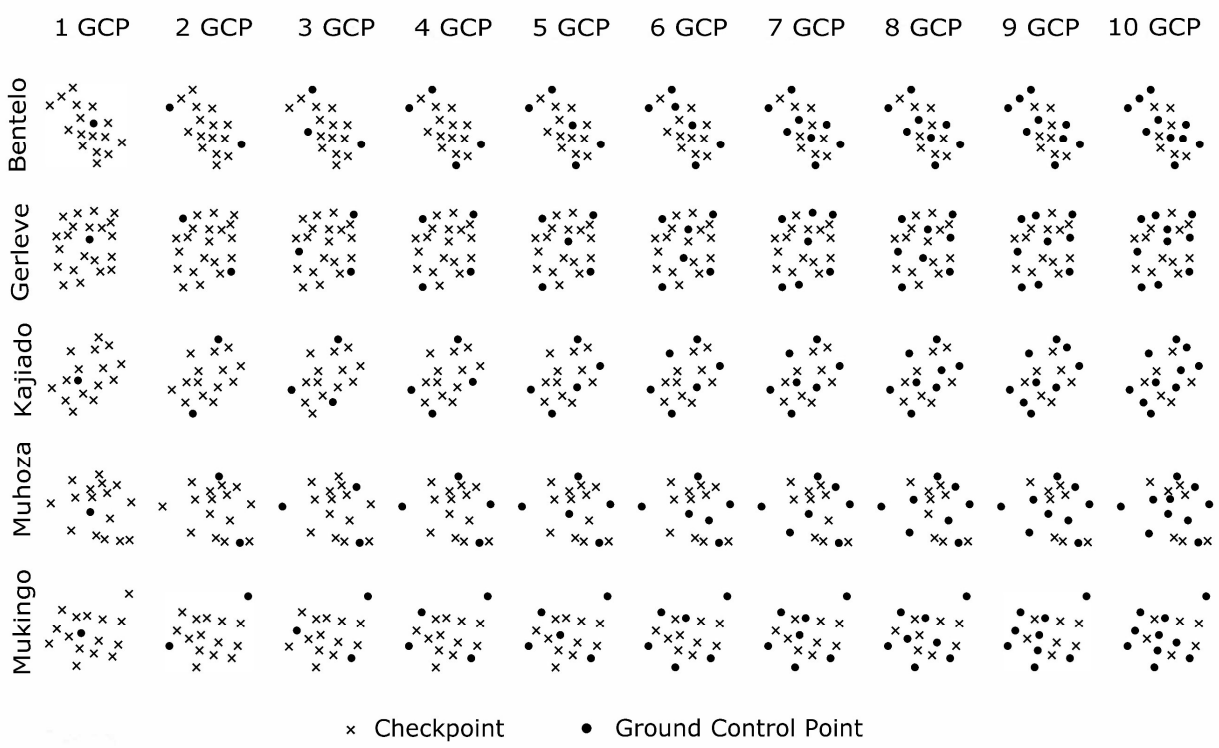

Figure 4.3: Distribution of GCPs for experimental assessment of the spatial accuracy

The final spatial accuracy was calculated during two different steps: firstly, the geometric error was determined after the BBA as outlined in the quality report of Pix4D. The horizontal error of a checkpoint was determined using the Euclidean distance of the residuals in $X$ and $Y$ directions. The residuals of the $Z$ coordinate represented the vertical offset. To also account for displacement errors during the orthophoto generation, this study secondly foresaw an accuracy assessment of checkpoint residuals in the final data product, as the absolute accuracy of points in the orthophoto, are of vital importance for cadastral surveying. Respective centre points were visually identified and marked in the orthomosaic using QGIS. Horizontal errors were derived by $X$ and $Y$ residuals, whereas the vertical error was extracted based on the raster value of the DSM. To describe the overall planimetric and vertical error of a particular processing scenario, the root mean square error (RMSE) was calculated following the ISO standard (ISO, 2013). In this context, the GNSS measurement of the checkpoint coordinate was treated as true value and the extracted coordinates from the orthophoto as the predicted value. 


\subsubsection{Estimating the impact of different flight plans on the characteristics of extracted cadastral features}

In contrast to the other two methods, the third quality evaluation utilizes only data from one regional context. Following basic photogrammetric principles, it is clear that the amount of image overlap significantly impacts the quality of the reconstructed scene. Thus, different flight patterns (with and without cross-flight), as well as different image overlap ( $50 \%$ and $75 \%$ forward overlap as well as $60 \%, 70 \%$ and $80 \%$ side lap) configurations, were exemplified for the study area Kibonde to ultimately show the impact of different flight plans on the reconstruction quality of cadastral features and subsequent automatic delineation results. Orthophotos were processed, and a quadratic shape of 500 by $500 \mathrm{~m}$ of the centre of Kibonde was chosen as an area of interest as required by the image segmentation algorithm (Crommelinck, 2019). To ultimately analyse geometric features and line discontinuities, this chapter foresaw a workflow including image segmentation algorithms as well as raster and vector operations, as shown in Figures 4.4 and 4.5. The first step was establishing reference lines and creating a mask to clip all candidate lines subject to this analysis. Reference lines were based on independently captured UAV images ( $80 \%$ forward overlap and side lap, cross-flight at a different altitude) and a resulting orthomosaic with $1.5 \mathrm{~cm}$ resolution. Two distinct features, namely building rooftops and concrete walls, were selected as representative visible objects important for cadastral applications. Both feature types were manually digitized and served as reference lines for subsequent analyses.

A uniform vector mask representing the vicinity of concrete walls and rooftops was created to minimize the number of candidate boundary lines. A slope layer was the basis for selecting a $1 \mathrm{~m}$ buffer of all raster cells of the DSM representing $>75 \%$ of the height gradient. Additionally, a vegetation mask was created to remove vegetated areas as those would negatively impact the straightness of selected cadastral features independent of the quality of the orthomosaic and thus would introduce unintended noise to the analysis of geometric discontinuities. The vegetation mask was based on the Green-Red Difference Index (GRDI). Raster cells above a GRDI of 0.02 were classified as vegetation and polygonised to calculate a buffer of $1 \mathrm{~m}$. Finally, the slope-based mask was clipped with the buffer of the GRDI to exclude vegetation from the samples. 


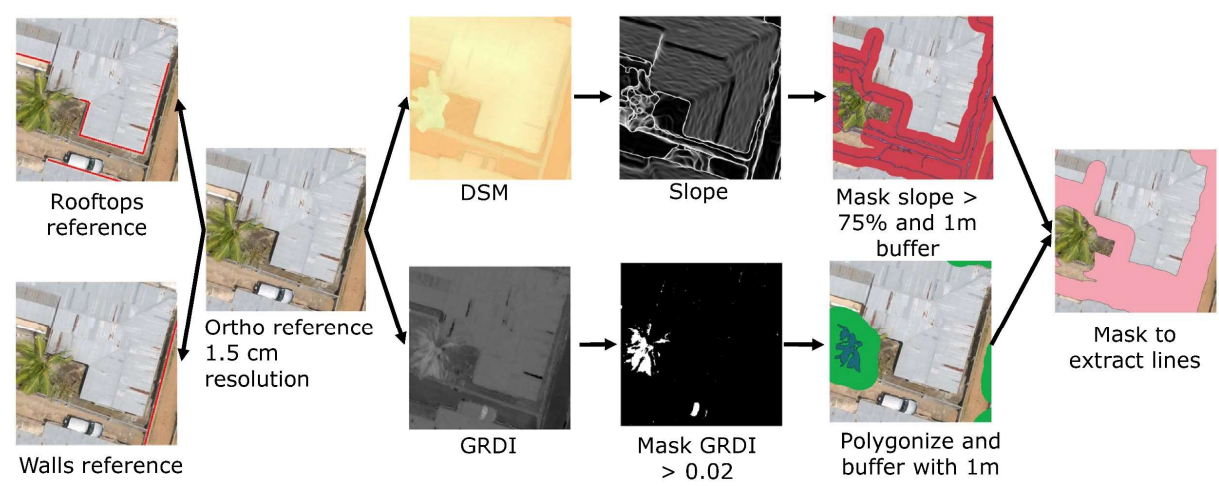

Figure 4.4: Workflow to define reference lines and a search mask for lines representing concrete walls and rooftops

In the second step, Multiscale Combinatorial Grouping (MCG) (PontTuset et al., 2017) was applied to all orthophotos to ultimately derive closed contour lines of visible objects, as suggested by (Crommelinck et al., 2019). The segmentation threshold was set to $k=0.6$ as this has proven to limit over-segmentation while still maintaining relevant cadastral objects in the context of this study. As shown in Figure 4.5, the resulting lines were polygonised and simplified according to (Crommelinck, 2019). Once the lines were clipped with the reference mask, several geometric and spatial characteristics were queried. Candidate lines were selected by overlaying the MCG lines with a 0.5 $\mathrm{m}$ buffer of reference lines. From those candidate lines, lines representing rooftops and walls were chosen manually. To calculate the correspondence and spatial difference to reference lines, the MCG lines representing walls and rooftops were split into segments of $10 \mathrm{~cm}$ and subsequently converted to points. Afterwards the distance from each point in the MCG line to the closest point of the reference line was calculated to derive statistical values for the spatial offset. To describe the amount of MCG lines that could automatically be extracted (i.e. correspondence with reference lines), a neighbourhood analysis was carried out to estimate the percentage of reference lines that could be reproduced by the MCG algorithm. As a last characteristic, this study calculated the sinuosity as a measure of the straightness of MCG lines to reflect on inconsistencies of critical features in the orthomosaics. Similar to the spatial offset, the sinuosity was calculated based on summed length of the MCG lines for one object in relation to the length of a virtual straight line (Figure 4.5). 


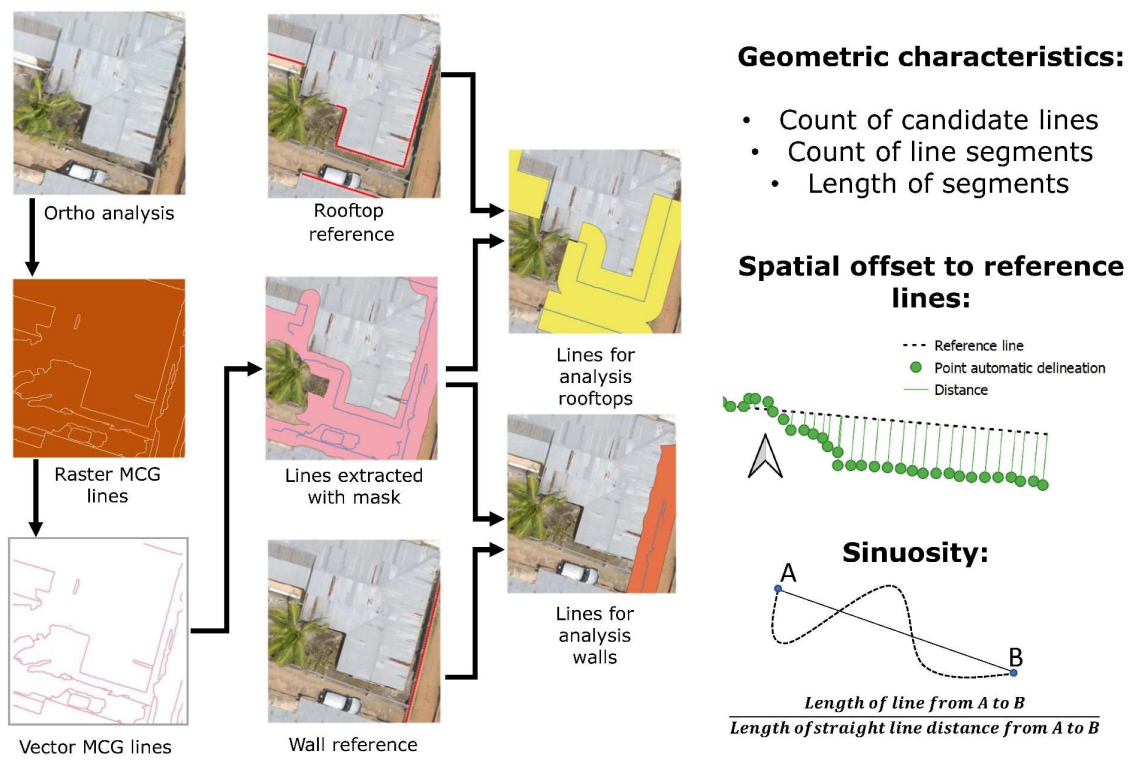

Figure 4.5: Workflow to compute and select MCG lines representing rooftops or walls and analytical tools to describe geometric characteristics of selected MCG lines.

\subsection{Results}

\subsubsection{Image matching: image correspondences}

The pairwise image correspondences were derived from comparing feature matching success rates representing certain land use classes prevalent in the images. The diagram in Figure 4.6 depicts standardized z-scores and mean values of automated tie-points for SIFT, SURF and AKAZE. At first glance, the results of various matching algorithms demonstrate a similar distribution, whereas apparent differences between land use classes are evident.

Image pairs characterized by forest and cultivated agricultural fields show significantly low numbers of automated tie-points. In some cases, no matches could be found. Images displaying non-cultivated agricultural field plots stick out by an extensive range of images correspondences for all three feature matching approaches. Here, the dataset Bentelo reaches the highest $\mathrm{z}$-scores, and the results are multiple standard deviations above the mean. However, insufficient numbers of automated tie-points are evident in this land use class, 
particularly for Gerleve. This can be ascribed to poor illumination conditions and little contrast in the images. The remaining datasets are clustered in a range between -0.5 to 1.5 of the $z$-score.

For image scenes showing human-made structures, two different trends are visible. The first trend describes the following correlation: On average, Kibonde, Muhoza and Mukingo indicate more key-point matches if less vegetation and more structures are prevalent. Thus, for Kibonde and Mukingo, a higher z-score was achieved with the periurban scene context compared to the rural context. The same applies to Muhoza with the land uses peri-urban and urban, respectively. In contrast, Kajiado does not follow this trend and represents the dataset with the highest z-scores for all three land use classes (rural, periurban, urban). The same applies to all three image matching algorithms. A possible explanation for this may be the climate zone. As indicated above, high vegetation presents an adverse condition for finding tie-points. In contrast to the humid climate in Kibonde, Mukingo and Muhoza, Kajiado is located in a semi-arid region characterized by sparse shrub and bush vegetation. Thus, the impact of vegetation is almost not visible and rural and urban scenes achieve similar z-scores.

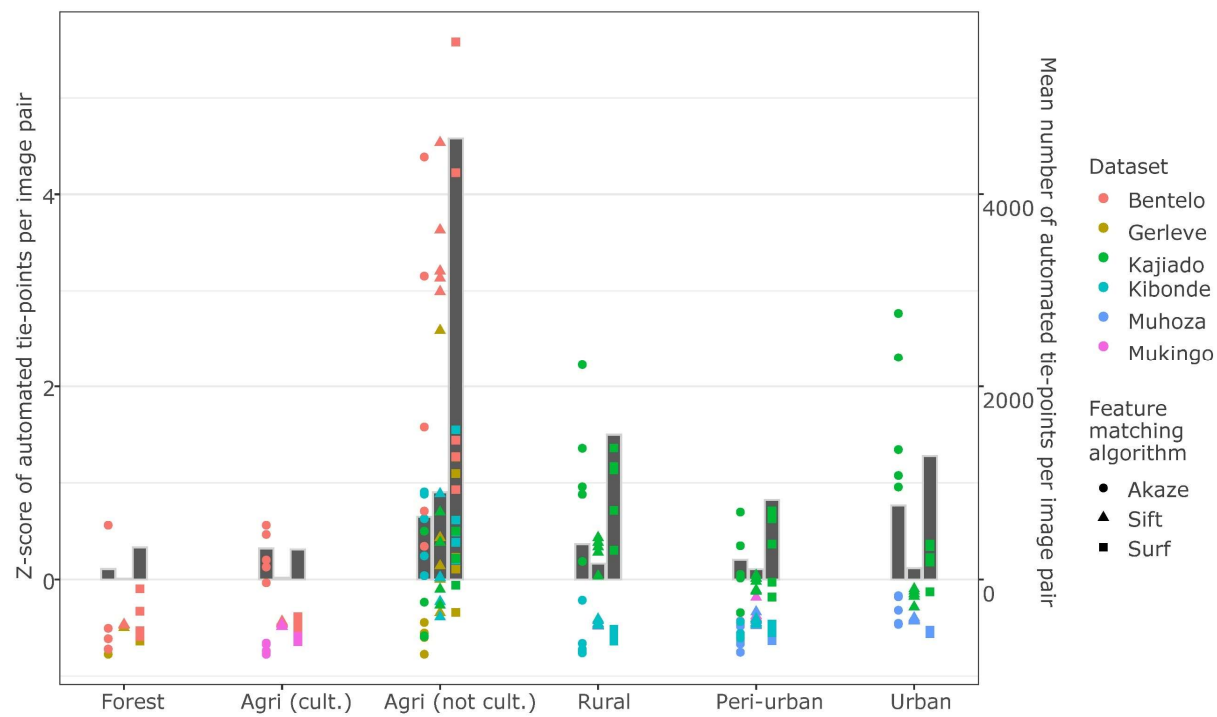

Figure 4.6: Standardized values of automated tie-points using SIFT, AKAZE, and SURF as feature extraction, detection and matching algorithm. The mean number of automated tie-points per algorithm and land use class is reflected as bars. The X-axis represents land use classes as defined in Table 4.3. 
Looking at the impact of image overlap on the automated tie-points in Table 4.4, it becomes clear that the poor feature matching results of forest can only be overcome with $90 \%$ image overlap while the other land use classes already show sufficient matches with less overlap. Similar to Figure 4.6, non-cultivated agricultural areas present the highest rate of image correspondences for all image overlap scenarios. Two adverse conditions could explain the poor rate of automated tiepoints in the forest. Firstly, although the flight is configured with a high image overlap, the difference in the viewing angle is larger between image points showing the tree's crown than for image objects on the ground. Thus, we observe that key points show insufficient similarity to be determined as image correspondence. This challenge can only be overcome by $80-90 \%$ image overlap. However, at the same time, the descriptors of leaves could also be too similar, leading to ambiguities during the feature matching process. Both effects are visible and could explain the comparatively low number of automated tie-points for all four image overlap configurations. In addition, and more or less independently from that, high vegetation cannot be regarded "static", which is an indispensable requirement for mono-camera bundle adjustment.

Table 4.4: Mean of automated tie-points of image pairs showing different land use classes and overlap

\begin{tabular}{lllll}
\hline & $\begin{array}{l}\mathbf{6 0 \%} \\
\text { overlap }\end{array}$ & $\begin{array}{l}\mathbf{7 0 \%} \\
\text { overlap }\end{array}$ & $\begin{array}{l}\mathbf{8 0 \%} \\
\text { overlap }\end{array}$ & $\begin{array}{l}\mathbf{9 0 \%} \\
\text { overlap }\end{array}$ \\
\hline Agriculture (not cultivated) & 289 & 666 & 2519 & $\mathrm{n} / \mathrm{a}$ \\
Rural & 83 & 116 & 291 & $\mathrm{n} / \mathrm{a}$ \\
Peri-urban & 18 & 302 & 326 & $\mathrm{n} / \mathrm{a}$ \\
Forest & 0 & 5 & 6 & 50 \\
\hline
\end{tabular}

\subsubsection{Absolute accuracy: Checkpoint residuals in DSM and orthophotos}

The absolute accuracy was determined after the bundle block adjustment as well as after the orthophoto generation. Figure 4.7 presents the RMSE of horizontal and vertical checkpoint residuals of all datasets. Looking at the results, it is evident that, in general, all datasets show a similar pattern. For photogrammetric processing with less than 5 GCPs, the resulting RMSE of the datasets differ widely. For results with more than 5 GCPs, the final RMSE seems to stabilize at a certain level. 
Looking at the horizontal RMSE, the large variance of the datasets for processing scenarios from 0 to 5 GCPs can be explained by the different quality of positional sensors. If no ground truth is included (0 GCPs), the BBA solely uses image geotags to estimate the absolute position of the reconstructed scene. Here, Gerleve was the only dataset with a professional PPK enabled GNSS device and attained the lowest RMSE (10 GSD) for all datasets processed with 0 GCP.

In contrast, Kajiado was flown with a consumer-grade UAV showing a large horizontal offset of more than 200 GSD. Bentelo, Mukingo and Muhoza achieve an RMSE between 50-100 GSD without GCPs, which is considered a typical error range of GNSS positioning without enhancement methods. Except for the dataset Mukingo, the RMSE drops significantly with including $1 \mathrm{GCP}$ as the systematic lateral shift can be corrected. For the scenario with $3 \mathrm{GCP}$, all datasets achieve a horizontal RMSE between 10-20 cm. Gerleve and Bentelo reach an RMSE of less than $10 \mathrm{~cm}$ after 6 GCPs and are followed by Kajiado and Muhoza after 7 GCPs. Subsequently, almost all datasets keep the same level alternating within a range of 1 GSD. In this aspect, Mukingo achieves the most accurate results with less than $5 \mathrm{~cm}$ RMSE after 5 GCPs. Muhoza is the only dataset that nearly improves its RMSE for each scenario that adds one more GCP.

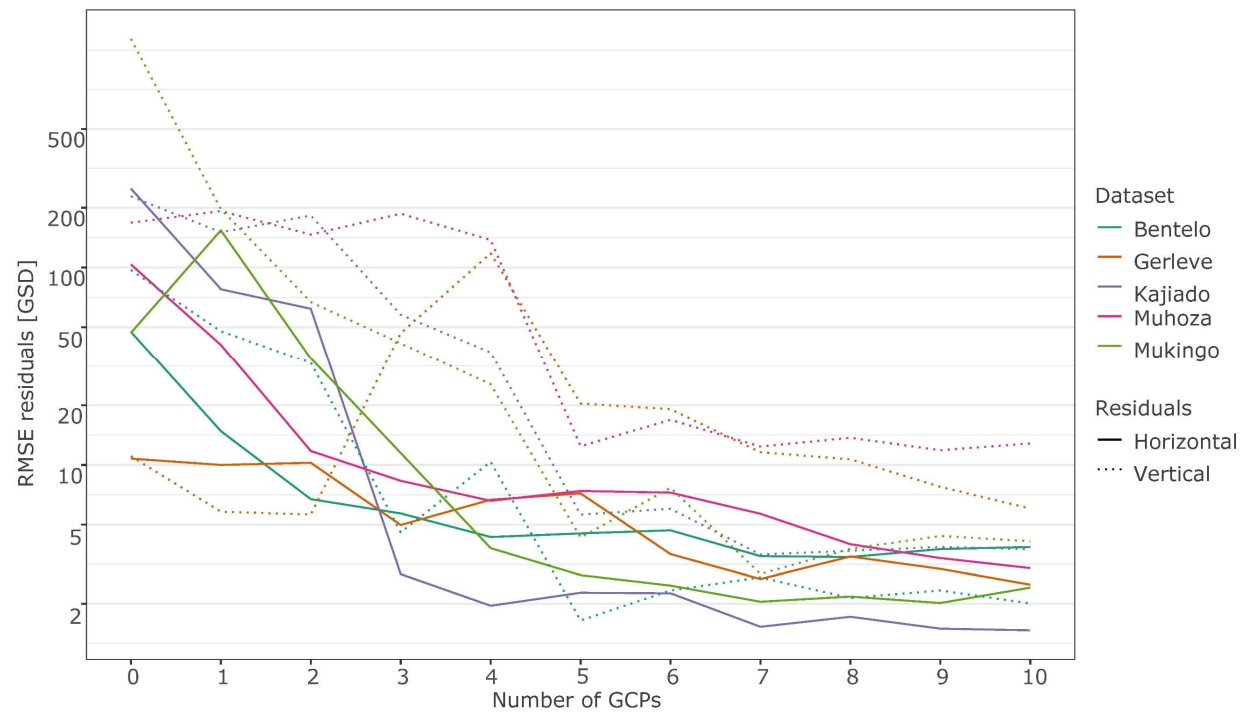

Figure 4.7: RMSE of checkpoint residuals measured in the DSM (vertical) and orthophoto (horizontal). 
Looking at the vertical residuals, Figure 4.7 suggests a higher dynamic compared to horizontal residuals. In general, residuals are larger than the values of the horizontal RMSE and start to level only after 7 GCPs. With a height offset of more than 1000 GSD, which corresponds to approximately $30 \mathrm{~m}$, the dataset Mukingo shows the maximum value without including GCPs. This can be attributed to a general definition problem of the height model used by DJI and can be corrected by adding at least 1 GCP. Similar to the horizontal residuals, Gerleve achieves the highest accuracy with an RMSE of only 10 GSD. However, after 2 GCPs, the height residuals abruptly increase before decreasing again after $4 \mathrm{GCP}$, indicating that this dataset requires a checkpoint in the centre of the scene to correct severe height deformations. At 5 GCPs, all datasets demonstrate a significant improvement of the vertical RMSE. Independent of the size of the area, five evenly distributed GCPs can be considered the minimum number of GCPs that efficiently fix cushion and dome deformations during scene reconstruction. After 7 GCPs, the vertical residuals of Bentelo, Kajiado, and Mukingo stabilize within the range of 1 GSD, whereas Muhoza and Gerleve continue to lower their RMSE.

Additional to the absolute accuracy, the difference of the RMSE after BBA to the RMSE after DSM and orthophoto generation are shown in Table 4.5. The presented values reveal insights about the share of the overall error, which accumulates after the BBA during the 3Dreconstruction and ortho-generation process, independent of horizontal or vertical displacement indicated during the BBA. Negative values suggest that the RMSE after the BBA is higher than the RMSE of the residuals taken from the DSM / orthophoto. On average, variations between the error measures remain very low (below 1 GSD) and do not show a clear trend of overestimating one or the other and no relation to the number of GCPs.

However, for Gerleve and Muhoza, horizontal residuals range up to 3 GSD, and for vertical residuals, we observe differences up to 5 GSD in two cases. For both datasets, significantly higher differences in the RMSE of checkpoint residuals could be explained by the challenging conditions for the 3D-reconstruction and orthophoto-generation processes. For Muhoza, difficulties could arise from considerable height (i.e. land surface) dynamics of the densely populated urbanized centre. 
Table 4.5: Differences of RMSE of checkpoint residuals measured after the $B B A$ and in the orthophoto/DSM. Values are normalized, according to GSD. Horizontal $(h)$ and vertical $(v)$ errors are treated separately. Differences $>1$ GSD are indicated in bold.

\begin{tabular}{|c|c|c|c|c|c|}
\hline & $\begin{array}{c}\text { Bentelo } \\
\text { h/v [GSD] }\end{array}$ & $\begin{array}{c}\text { Gerleve } \\
\text { h/v [GSD] }\end{array}$ & $\begin{array}{c}\text { Kajiado } \\
\text { h/v [GSD] }\end{array}$ & $\begin{array}{c}\text { Muhoza } \\
\text { h/v [GSD] }\end{array}$ & $\begin{array}{c}\text { Mukingo } \\
\text { h/v [GSD] }\end{array}$ \\
\hline $0 \mathrm{GCP}$ & $0.39 /-1.96$ & $-0.03 /-0.84$ & $0.05 /-0.45$ & $0.29 / 0.69$ & $0.38 / 0.22$ \\
\hline $1 \mathrm{GCP}$ & $-0.11 /-0.08$ & $0.05 / 0.73$ & $-0.09 /-0.10$ & $-0.01 / 3.79$ & $-0.31 /-0.02$ \\
\hline $2 \mathrm{GCP}$ & $0.37 / 0.07$ & $-0.02 /-0.11$ & $-0.24 /-0.18$ & $0.01 / 2.72$ & $-0.28 /-0.22$ \\
\hline 3 GCP & $0.05 /-0.28$ & $-0.19 / 0.20$ & $0.18 /-0.57$ & $0.72 / 1.07$ & $0.24 / 0.16$ \\
\hline 4 GCP & $0.15 /-0.52$ & 2.11/-0.72 & $0.23 / 0.94$ & $0.60 /-4.12$ & $-0.27 / 0.49$ \\
\hline 5 GCP & $0.10 / 0.01$ & $2.17 /-1.09$ & $0.44 /-0.24$ & 2.81/0.18 & $0.15 /-0.34$ \\
\hline $6 \mathrm{GCP}$ & $0.15 / 0.05$ & $-0.15 /-0.55$ & $0.58 /-0.37$ & $\mathbf{1 . 6 0} / 0.18$ & $0.19 / 0.16$ \\
\hline 7 GCP & $-0.13 /-0.35$ & $-0.05 / 0.57$ & $0.24 /-0.70$ & $1.42 /-0.12$ & $0.23 /-0.23$ \\
\hline $8 \mathrm{GCP}$ & $-0.08 /-0.22$ & $-0.03 / 1.63$ & $0.35 /-0.37$ & $-0.81 / 0.41$ & $0.27 /-0.07$ \\
\hline 9 GCP & $-0.22 /-0.31$ & $-1.55 /-1.02$ & $0.29 / 0.39$ & $-0.50 /-4.89$ & $0.11 /-0.66$ \\
\hline $10 \mathrm{GCP}$ & $-0.11 /-0.31$ & $0.42 / 0.68$ & $0.17 /-0.02$ & $-0.24 /-2.60$ & $0.28 /-0.05$ \\
\hline
\end{tabular}

\subsubsection{Relative accuracy: Characteristics of automatically extracted cadastral features}

Various line geometry measures present the quality of the scene reconstruction and subsequent feature extraction. For the chosen quadratic scene in the centre of the Kibonde dataset, houses are predominantly covered by corrugated iron roofs and parcels are usually separated by concrete walls or bushes. To minimize external noise to our statistical assessment, only walls and rooftops without the interference of vegetation were delineated as reference (Figure 4.8). This adds up to a total of $692.3 \mathrm{~m}$ of lines referring to rooftops and $196.4 \mathrm{~m}$ of lines representing walls. As presented in Table 4.6, this relation is also expressed by candidate lines counted in a $0.5 \mathrm{~m}$ buffer of all reference lines.

Interestingly, the impact of the flight pattern (cross-flight or no crossflight) is more evident for rooftops than for walls, shown by the difference of line counts for different flight pattern scenarios. Concerning reference walls, marginally (within 10\% range) fewer candidate lines were selected compared to the same scenario without a cross-flight pattern. In contrast, for rooftops, $10 \%-40 \%$ fewer candidate lines were identified for processing scenarios considering a cross-flight pattern. 


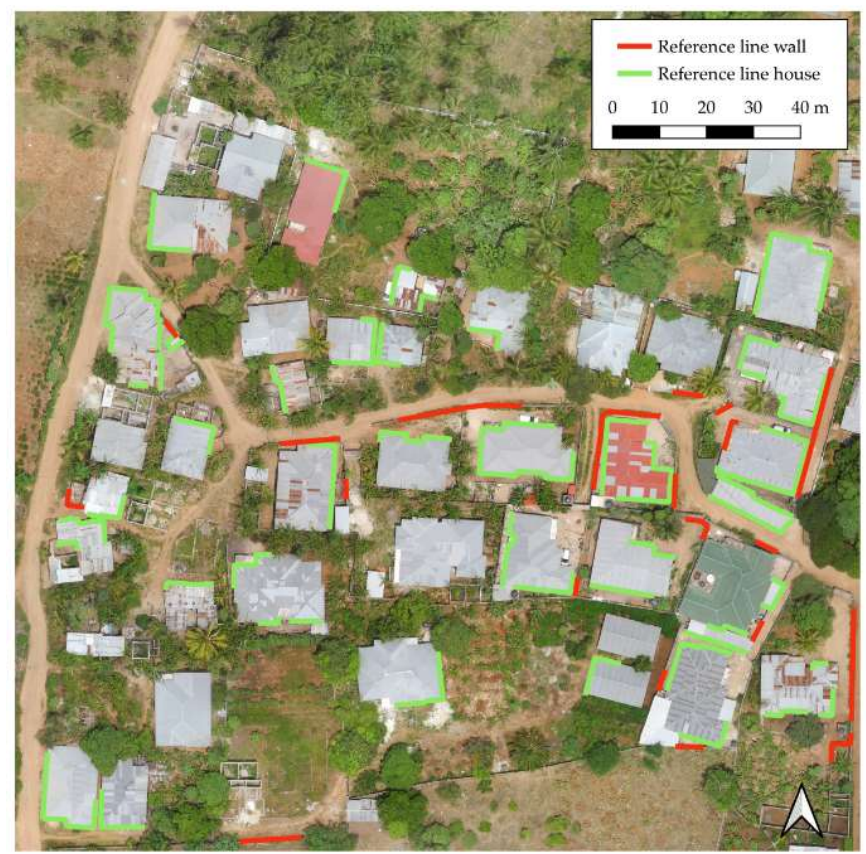

Figure 4.8: Selected reference lines representing rooftops (green) and walls (red) for the AOI in Kibonde

Looking at the count of selected line segments, a more homogenous picture can be drawn. In all cases, the line count for the cross-flight pattern is lower than for the same image overlap scenario without a cross-flight. The mean length of line segments shows no significant difference between walls and rooftops. However, an important observation can be made concerning the image overlap. On average, line segments are shorter for scenarios with only $50 \%$ forward overlap than flight plans with $75 \%$ overlap. Combining a higher count of line segments and a smaller average line length proves a higher fragmentation of boundary features for orthophotos without a cross flight pattern and lower image overlap scenarios. This result becomes even more apparent concerning the correlation of selected MCG lines with the reference dataset. Here, the improvement of the correlation with reference lines is more significant for walls than for rooftops. In this aspect, walls demonstrate a range between $71.5 \%$ to $93 \%$ and steadily increase with higher image overlap (both forward and side lap). This means the MCG algorithm applied to the orthophoto generated with a poor flight plan produces contours for only $71.5 \%$ of the walls. In contrast, an orthophoto based on a favourable flight plan achieves an object detection rate of $93 \%$. Hence, the detection range 
of contour lines for rooftops is comparatively small, with a maximal $2.9 \%$ variance between different flight plan scenarios.

A similar observation is evident for the sinuosity. Here, rooftops do not differ much, and lines of rooftops are on average 1.5 times longer than a perfectly straight line from the start to the endpoint. MCG lines representing walls are on average more curved and show a clear trend concerning the flight parameters reaching a minimal curviness with a cross flight pattern and $75 \%$ forward overlap and $80 \%$ side lap. Particularly for lines representing rooftops, it should be noted that the sinuosity values are relatively high due to the origin of the MCG lines, which were created based on a raster dataset and consequently still show undulations at the pixel level.

Table 4.6.: Qualitative and quantitative characteristics of line geometries representing rooftops $(R)$ and walls $(W)$ separated according to flight configuration (forward overlap [\%], side lap [\%]) and flight pattern (CF= cross flight pattern, no CF = no cross-flight pattern). Min. and max. are bold.

\begin{tabular}{|c|c|c|c|c|c|c|c|}
\hline \multirow[t]{2}{*}{ Overlap [f-s] } & & \multicolumn{2}{|c|}{$50 \%-60 \%$} & \multicolumn{2}{|c|}{$50 \%-70 \%$} & \multicolumn{2}{|c|}{$50 \%-80 \%$} \\
\hline & & no CF & CF & no CF & CF & no CF & $\mathbf{C F}$ \\
\hline Candidate lines & W & 144 & 146 & 121 & 133 & 177 & 157 \\
\hline $0.5 \mathrm{~m}$ buffer [count] & $\mathrm{R}$ & 333 & 273 & 410 & 285 & 505 & 310 \\
\hline Selected line & w & 73 & 42 & 76 & 63 & 67 & 50 \\
\hline segments [count] & $\mathrm{R}$ & 209 & 177 & 233 & 180 & 256 & 180 \\
\hline \multirow{4}{*}{$\begin{array}{l}\text { Mean length of line } \\
\text { segments }[\mathrm{m}] \\
\text { Correspondence with } \\
\text { reference }[\%]\end{array}$} & W & 3.22 & 4.68 & 3.50 & 3.39 & 3.32 & 4.96 \\
\hline & $\mathrm{R}$ & 3.65 & 4.37 & 3.34 & 4.28 & 3.01 & 4.21 \\
\hline & W & 71.5 & 85.8 & 79.0 & 90.1 & 82.5 & 87.8 \\
\hline & $\mathrm{R}$ & 94.9 & 95.8 & 94.8 & 95.1 & 96.6 & 95.6 \\
\hline \multirow{2}{*}{ Sinuosity } & W & 1.77 & 1.78 & 1.76 & 1.70 & 1.68 & 1.65 \\
\hline & $\mathrm{R}$ & 1.58 & 1.59 & 1.58 & 1.60 & 1.59 & 1.60 \\
\hline \multirow[t]{2}{*}{ Overlap [f-s] } & & \multicolumn{2}{|c|}{$75 \%-60 \%$} & \multicolumn{2}{|c|}{$75 \%-70 \%$} & \multicolumn{2}{|c|}{$75 \%-80 \%$} \\
\hline & & no CF & CF & no CF & CF & no CF & CF \\
\hline Candi & W & 158 & 122 & 165 & 133 & 129 & 134 \\
\hline $0.5 \mathrm{~m}$ buffer [count] & $\mathrm{R}$ & 402 & 271 & 366 & 295 & 444 & 369 \\
\hline \multirow{2}{*}{$\begin{array}{l}\text { Selected line } \\
\text { segments [count] }\end{array}$} & w & 78 & 44 & 75 & 57 & 54 & 40 \\
\hline & $\mathrm{R}$ & 243 & 161 & 189 & 168 & 220 & 173 \\
\hline \multirow{2}{*}{$\begin{array}{l}\text { Mean length of line } \\
\text { segments }[\mathrm{m}]\end{array}$} & W & 3.31 & 3.71 & 3.05 & 3.86 & 4.37 & 5.47 \\
\hline & $R$ & 3.24 & 4.87 & 4.14 & 4.54 & 4.40 & 4.50 \\
\hline \multirow{2}{*}{$\begin{array}{l}\text { Correspondence with } \\
\text { reference }[\%]\end{array}$} & w & 88.5 & 83.6 & 93.0 & 87.6 & 91.9 & 93.0 \\
\hline & $\mathrm{R}$ & 96.3 & 95.9 & 95.1 & 95.7 & 97.2 & 97.8 \\
\hline \multirow{2}{*}{ Sinuosity } & w & 1.66 & 1.62 & 1.74 & 1.62 & 1.66 & 1.58 \\
\hline & $\mathrm{R}$ & 1.60 & 1.61 & 1.58 & 1.59 & 1.61 & 1.58 \\
\hline
\end{tabular}


Aside from line feature characteristics, the spatial correlation was also investigated in terms of distance measurements of MCG lines to reference lines. Figure 4.9 visualizes the results and exemplifies the spatial correlation with a small sample of the entire dataset. Rooftops are mainly delineated close to the reference line, whereas walls show considerable variability. For example, we included the orthophoto generated with the poorest image overlap at the bottom of Figure 4.9. The visual interpretation reveals a significant deformation and poor orthorectification of the wall, leading to the displacement of MCG lines for the dataset with $50 \%$ 60\% overlap and without a cross-flight.

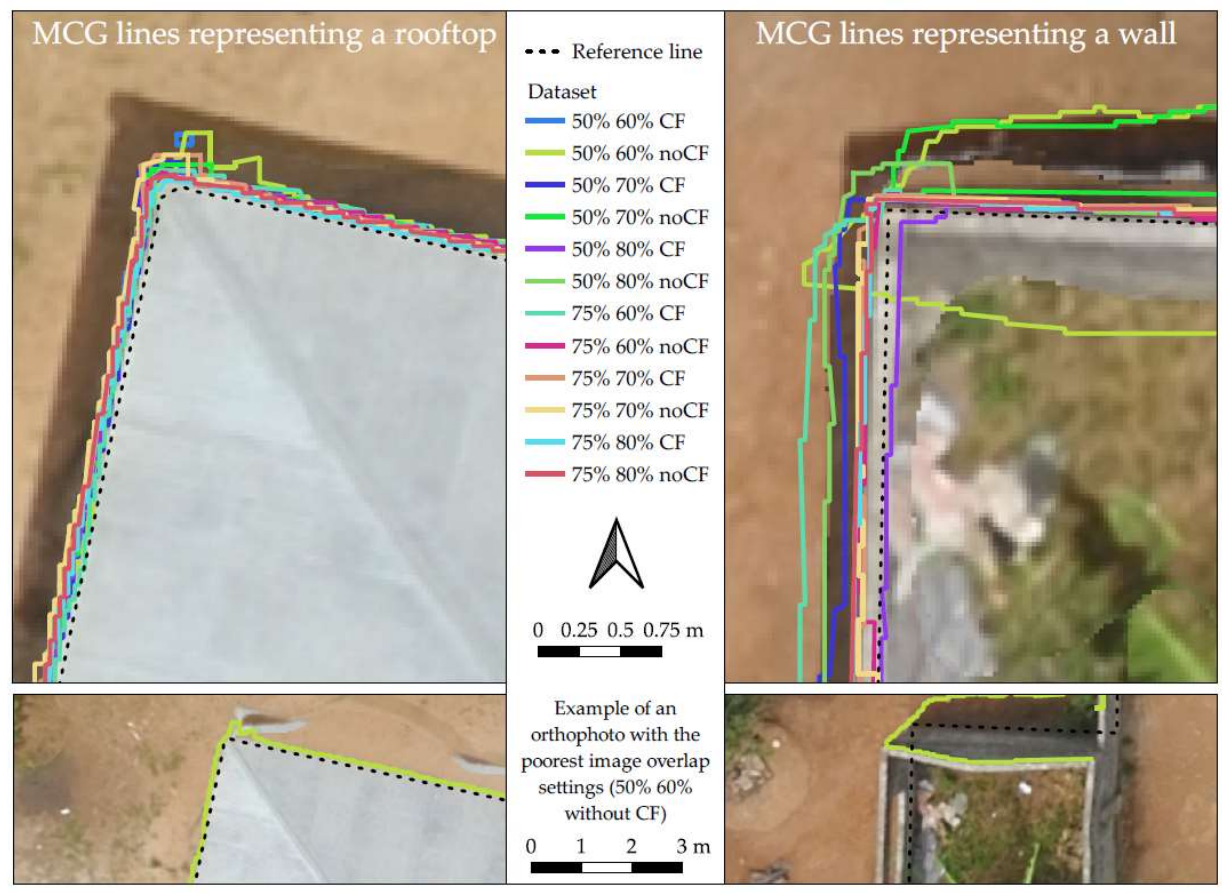

Figure 4.9: Example showing the differences of automatically extracted rooftops and walls separated according to flight configuration (forward overlap $[\%]$, side lap [\%]) and flight pattern (CF= cross flight, noCF = no cross flight).

This variability is also apparent in the statistics of the point-to-line distances, presented as Box-Whisker plots in Figure 4.10. The interquartile range of rooftops is significantly smaller than the one of the walls. It should be noted that the distances of reference walls are subject to a systematic offset of $15 \mathrm{~cm}$ as the reference line was placed in the centre of the wall, whereas the MCG algorithm produced lines on the right or left edge. 


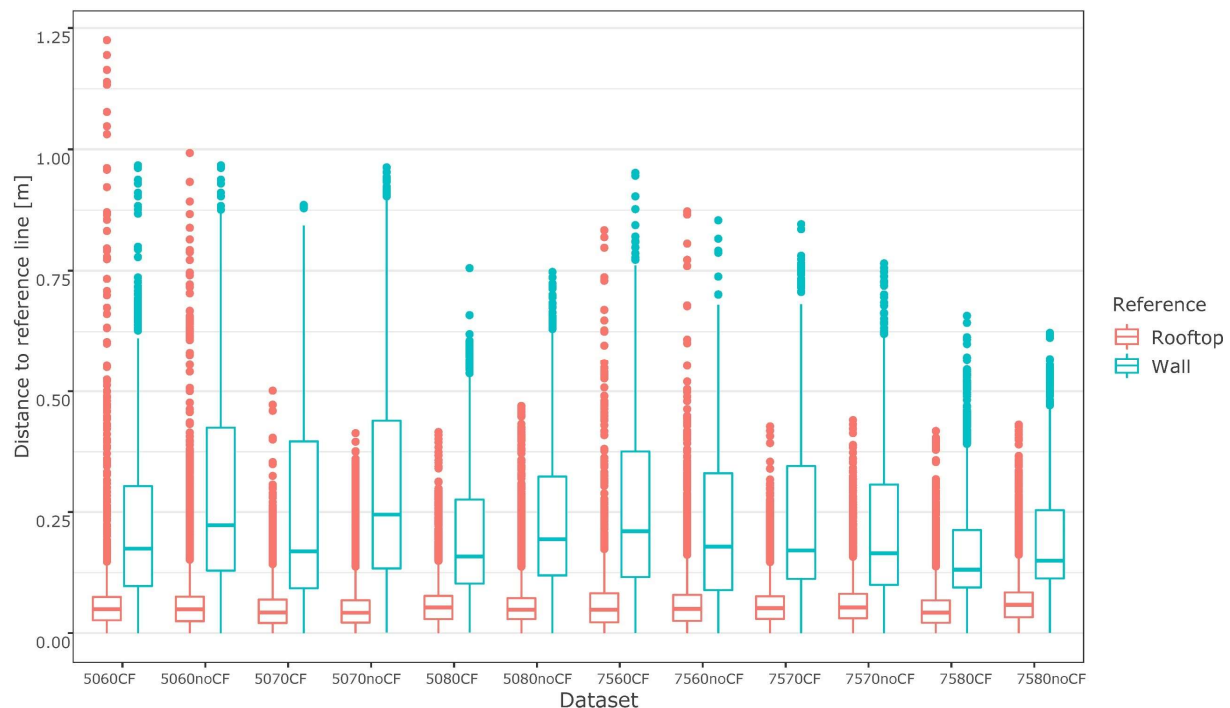

Figure 4.10: Box-Whisker plot of point distances to reference lines separated according to the reference wall and rooftop. Box represents the interquartile range (IQR) with the median; whisker represents 1.5 IQR, points represent outliers. X-axes label refers to flight parameter, e.g. 5060CF means 50\% forward overlap, 60\% side lap and cross-flight (CF) pattern. Distances reflect the length of perpendicular lines from points to reference lines. Points were created every $10 \mathrm{~cm}$ from a line geometry derived by feature extraction with the MCG algorithm.

For two flight scenarios with low overlap, outliers of point distances of rooftops exceed the outliers of walls. In general, the share of outliers is higher for rooftops than for walls indicating that almost all rooftops are delineated in a range of approximately $20 \mathrm{~cm}$ with a few extreme variations. For wall features, the statistical analysis confirms the observations from the line characteristics, showing that the overall quality of delineated walls differs highly concerning the image overlap and flight plan settings. Best results represented by the lowest fivenumber values of the Box-Whisker plot were returned for flight scenarios with $75 \%$ forward overlap, $80 \%$ side lap, and a cross flight pattern.

As evident in Figure 4.11, the RMSE of horizontal checkpoint residuals of the orthophoto stays between 0.8-1.5 GSD for all flight configurations, corresponding to $2.5-4 \mathrm{~cm}$. Similar to Figure 4.9, the statistics of the offset of detected line features show a noticeable discrepancy between rooftops $(<10 \mathrm{~cm})$ and walls $(20-40 \mathrm{~cm})$. 


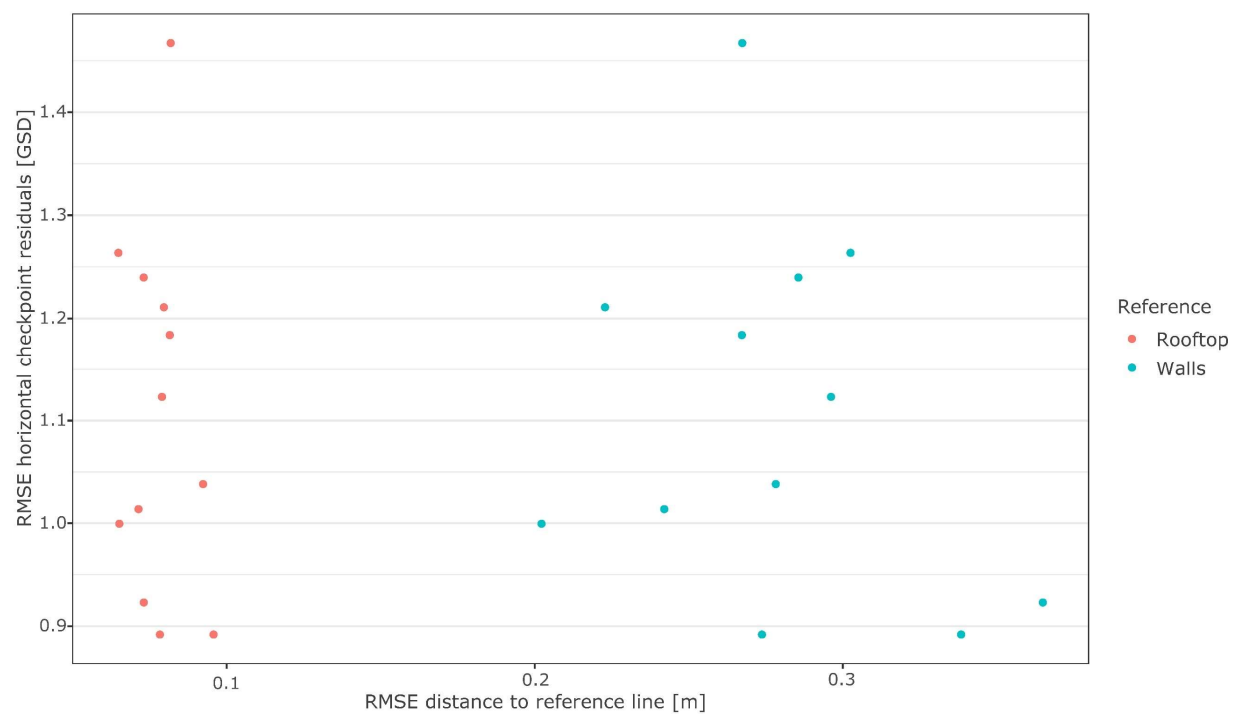

Figure 4.11: Scatterplot of error metrics for delineated rooftops and walls of orthophotos captured with different flight configurations. Absolute accuracy of the orthophoto is given on the $y$-axis with the RMSE of horizontal checkpoint residuals. Relative accuracy is shown on the $x$-axis given by the RMSE of point distances to reference lines. Note that both axes have different scales.

In contrast to checkpoint residuals of the orthophoto, which do not correlate with the error metrics, Figure 4.12 reveals that the flight pattern has implications on the relative accuracy of extracted features. Walls directed perpendicular to the flight direction show almost the same statistics for both scenarios, with a cross-flight or without a cross-flight pattern. However, a cross-flight pattern for walls parallel to the flight direction improves the results indicated by a lower median and a smaller IQR. This result could be attributed to the fact that geometries of features parallel to epipolar lines imply more challenges to correctly estimate the 3D position and subsequent image matching and ortho-generation. 


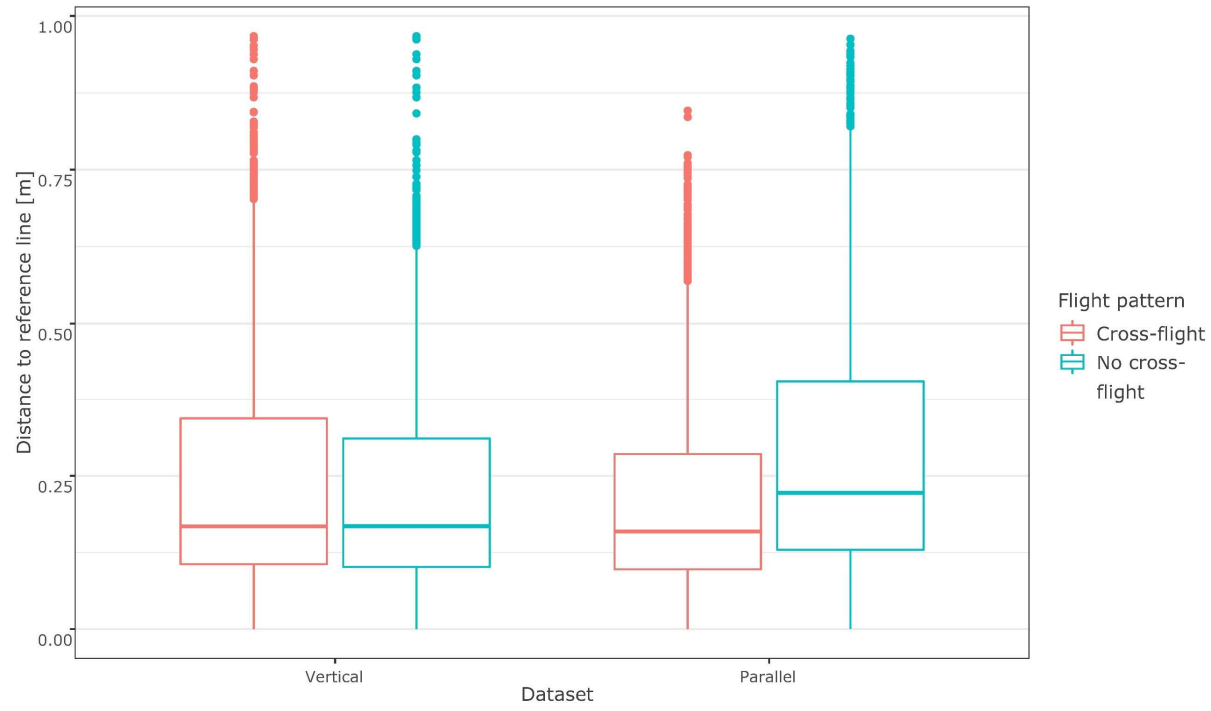

Figure 4.12: Box-Whisker plot of distances to reference lines separated according to the direction of walls (parallel or vertical to the flight direction). Box represents the interquartile range (IQR) with the median; whisker represent $1.5 I Q R$, points represent outliers.

\subsection{Discussion}

Even though UAV can collect images with a resolution of a few centimetres, results in this chapter show that the absolute and relative accuracy can differ from some centimetres up to several meters depending on the chosen flight configuration. To exploit the full potential of UAV-based workflows for land administration tasks, careful decisions on efficient mission planning are essential. This holds for both sides: collecting as many images and GCPs as needed to meet the expected survey accuracy and collecting just as many images and GCPs as necessary to minimize computational costs in favour of time constraints or potential hardware limitations.

Several reports have shown that the quantity of automated tie-points impacts the quality of the photogrammetric 3D reconstruction as image correspondences are fundamental for accurately estimating image orientation parameters. Even though different sensors, UAVs, scenes and flight conditions were analysed in this chapter, a homogenous picture can be drawn when looking at generated tie-points concerning land use classes. In image scenes showing trees or crops, significantly lower rates of tie-points could be extracted compared to scenes with human-made structures or grassland. In the former case, only a high 
image overlap of at least $80-90 \%$ is sufficient to achieve an adequate number of image correspondences. These results match those reported in (Seifert et al., 2019). Most rural and peri-urban scenes are also characterized by vegetated areas subject to subsistence farming sideby-side to residential buildings in tropical or subtropical regions. Thus, an optimal flight mission might need to be configured with higher image overlap than a flight mission in arid or semi-arid regions.

Although we can show a clear correlation of generated tie-points and land use, the results suggest that the optimal number of GCPs seems to be independent of the climate zone or land cover, as all datasets in this analysis show a similar pattern and indicate no significant changes of the RMSE after seven equally distributed GCPs. This result reflects those of (Mesas-Carrascosa et al., 2015; Tonkin and Midgley, 2016; Agüera-Vega, Carvajal-Ramírez and Martínez-Carricondo, 2017) who also observed no significant differences in the final vertical or horizontal RMSE after 5 or 6 GCPs, respectively. In contrast to earlier findings (Sanz-Ablanedo et al., 2018), no evidence of the impact of the GCPs density was detected. In terms of GSD and despite considerably different study areas, all datasets in this analysis reach a similar error level with 10 GCPs, 2-3 GSD for the horizontal accuracy and 2-4 GSD for the vertical accuracy. Thus, the number and distribution of GCPs might play a more critical role than the density of GCPs. This is particularly interesting for the mission planning and cost calculations, as placing, marking, and measuring GCPs is one of the most timeconsuming and costly aspects of the entire UAV-based data collection campaign. In the case of Mukingo, we observed a substantial offset of the terrain height in the scenario without GCPs. This magnitude of vertical offset was already reported before (Manfreda et al., 2019) and seemed to be specific to DJI UAV.

Contrary to most other studies investigating checkpoint residuals, this analysis presents the absolute accuracy concerning the residuals after the BBA and in the final DSM and orthophoto. For two out of six datasets, our results show significant discrepancies between the checkpoint residuals with a magnitude of up to 5 GSD. In both cases, challenging conditions were present, i.e. poor illumination conditions for Gerleve and a densely populated built-up area in Muhoza. We observe that particular the height component could be strongly impacted. Consequently, considering checkpoint residuals measured in the orthophoto is indispensable for evaluating the final accuracy, as additional offsets might be introduced during the 3D reconstruction and orthophoto-generation process. 
As a third central aspect, this study reveals yet another perspective on the orthophoto quality: success rates of the automated extraction of cadastral features. Here, our findings point to a clear difference between the delineation of rooftops and walls. Whereas various flight configurations showed less impact on the extractability of rooftops, the automatic extraction of walls achieves more accurate and complete lines with large image overlaps and a cross-flight pattern. Even though the absolute difference of the correlation seems minor in our example, values of either $70 \%$ correlation or $93 \%$ correlation with reference lines are significant for scaled applications. A smaller percentage would entail a lot more manual work of delineating respective walls that were not represented by MCG lines. Furthermore, the MCG algorithm applied on an orthophoto of a weak image block - as described by lower image overlap - produces shorter line segments which also implies more manual effort to receive a complete delineation finally. Thus, thoughts should also be given to characteristics of extractable features when designing a UAV flight mission. Our findings suggest that planar cadastral features are less sensitive to differences in flight configurations than thin image objects such as walls or fences. Consequently, the latter necessitates a larger image overlap to be reliably reconstructed and detectable during subsequent automatic delineation processing. Additionally, a cross flight pattern is clearly recommendable when thin cadastral objects are oriented towards different cardinal directions.

In combination, the results are significant in at least two aspects. Firstly, although we investigated very different study sites, common trends are evident, drawing some general recommendations as outlined below. Independent of the sensor or feature matching algorithm, vegetated spaces, and forests or cultivated agricultural areas still present challenges to establishing image correspondences. However, findings of checkpoint residuals suggest that the impact on the overall accuracy is only marginal when looking at scenes with multiple land use classes.

Secondly, the research investigations reveal discrepancies between the spatial accuracy and the completeness of automatically detected cadastral features, even though the RMSE of the orthophoto as a commonly accepted error measure is low. According to these data, we can infer that the flight configurations play a crucial role in achieving high data quality, particularly for cadastral features characterized by height differences and thin shapes as exemplified for concrete walls. Moreover, in most cases, checkpoints are put out in open and visible spaces which do not necessarily reflect objects subject to manual or 
automatic cadastral delineation. Consequently, it should be emphasized that context-driven error analysis is essential to assess the overall accuracy of UAV-based data products.

Finally, a multifactorial analysis, as presented in this chapter, includes shortcomings on various ends. Checkpoints were distributed, ensuring high visibility, and the surrounding of most checkpoints was characterized by open space. Following good survey practice, no checkpoints were put under trees, close to houses or in densely vegetated areas. Thus, an analysis of the implications of poor feature matching results on the checkpoint residuals was not possible with our datasets. Furthermore, it should be emphasized that all datasets were collected following flight plans as specified in section 2 and the transferability of our findings to flight configurations in other contexts is questionable.

\subsection{Conclusion}

This chapter provides recommendations on optimal UAV data collection workflows for cadastral mapping based on a comprehensive analysis of data quality measures applied to numerous orthophotos generated from various flight configurations. Methods covered several aspects ranging from statistics on automated tie-points and an evaluation of the geometric accuracy to characteristics of automatically delineated cadastral features. The results highlight that scene context, flight configuration, and GCP setup significantly impact the final data quality of resulting orthophotos and subsequent automatic extraction of relevant cadastral features. In a nutshell, the following recommendations can be drawn:

1) Land use has a significant impact on the generation of tiepoints. Image scenes characterized by a high percentage of vegetated areas, especially trees or forests, require image overlap settings of at least $80 \%$ to $90 \%$ to establish sufficient image correspondences.

2) Independent of the size of the study area, the error level of planimetric and vertical residuals remains steady after seven equally distributed $\mathrm{GCPs}^{7}$, given at least $70 \%$ forward overlap and $70 \%$ side lap. As the absolute accuracy does not increase significantly with adding more GCPs, 7 GCPs can be recommended as optimal survey design.

\footnotetext{
${ }^{7}$ According to the scheme presented in Figure 4.3
} 
3) As exemplified for concrete walls, the quality of reconstructed thin cadastral objects is highly variable to the flight configuration. A large image overlap and a cross-flight pattern have proven to enhance the reliability of the generated orthophoto as quantified by the increased accuracy and completeness of automatically delineated walls. In contrast, the delineation results of rooftops showed less sensitivity to the flight configuration.

4) Even though checkpoint residuals indicate high absolute accuracy of an orthophoto, the reliability of reconstructed scene objects could vary, particularly in adverse conditions with large variations in the height component. We furthermore recommend measuring checkpoint residuals in the generated orthophoto in addition to after the BBA.

Generally, these findings have important implications for developing UAV-based workflows for land administration tasks. The fact that the data quality can significantly change depending on the flight configurations involves risks and opportunities. The risk is that UAVs are used as off the shelf products with little knowledge of photogrammetric principles and options to customize flight configurations. Consequently, even though the end-product appears to be of good quality, spatial offsets, deformations, or poor reconstruction results of relevant features might be present but remain undetected. However, at the same time, we also realize immense opportunities in the customization of UAV workflows. The results in this analysis show that different flight configurations and various ground-truthing measures offer a wide range of options to tailor the data collection task to financial, personnel and time capacities and optimally align it to customer needs and requirements in the land sector.

Future research building upon our results could follow different pathways. Although our study foresaw six different contexts, the terrain was mostly flat or slightly undulated and showed only minor surface variations. It would be interesting to explore if data of hilly and larger study areas could substantiate our recommendations. Secondly, this study neglects the ground sampling distance as a variable in our assessment. It is expected that next to the orthophoto quality also variations in the resolution significantly impact the completeness and accuracy of automatically extracted line geometries. Clues on this correlation could expand best-practice examples by adding recommendations on camera specifications and flight heights. 


\section{Chapter 5 - Scaling up UAVs in Land Administration: Towards the Plateau of Productivity ${ }^{8}$}

\section{This chapter is based on:}

Stöcker, C., Bennett, R., Koeva, M., Nex, F., Zevenbergen, J. Scaling up UAVs for land administration: towards the plateau of productivity. In: Land Use Policy (undergoing minor revision). 


\subsection{Introduction}

Providing equitable land and resource access for all people by 2030 is implicit and explicit to multiple targets of the Sustainable Development Goals promulgated by the United Nations (UN, 2015). To achieve this global plan, it is recognized there needs to be a rethink of conventional land surveying and mapping practices, often rooted in western-style land administration systems. With current rates and methods, it is estimated that it will take many decades, if not centuries, to achieve global coverage to provide tenure security for all (Zevenbergen et al., 2013). Researchers and practitioners worldwide have developed various innovative approaches and technologies covering spatial, legal, and institutional frameworks to tackle the challenge. Pilot studies have been carried out in different land administration contexts to test the validity of those innovations, such as remote sensing technologies (Santos, Fletschner and Daconto, 2014; Stöcker, Ho, et al., 2019; Koeva et al., 2020), participatory mapping (Asiama, Bennett and Zevenbergen, 2017; Aditya et al., 2020), geo-cloud processing (Koeva et al., 2021), fit-for-purpose land administration (Enemark et al., 2014), pro-poor land administration (Zevenbergen et al., 2013; Hendriks et al., 2019), and the continuum of land rights (UN-Habitat and GLTN, 2015), amongst others.

Amongst those tools, unmanned aerial vehicles (UAVs) are considered a viable technology to capture aerial images, which may even have the ability to revolutionise the toolset for land administration tasks (Rubinov et al., 2015) in various specific contexts. Compared to traditional labour-intensive in-field surveys with geodetic equipment, UAVs offer the opportunity to collect high-resolution and accurate data (Stöcker et al., 2020) for small to medium-sized areas (few ha to a few $\mathrm{km}^{2}$ ) in a time-efficient workflow. This allows for the timely supply of geospatial products as base data for various land administration processes. Furthermore, the data provided by UAVs have been widely applied to derive insights on land use, land development, land value or land tenure, either during participatory mapping activities (Barnes and Volkmann, 2015; Mumbone et al., 2015; Ramadhani, Bennett and Nex, 2018; Stöcker, Koeva and Zevenbergen, 2020) or as input data for automatic scene understanding procedures and machine learning ( $\mathrm{Yu}$ and Zhang, 2015; Crommelinck et al., 2018; Fetai et al., 2019; Xia, Persello and Koeva, 2019).

Looking at the prospects of UAV technology from a global perspective, there has been hype and excitement around UAVs as a disruptor for cargo transport and professional applications (Freeman and Freeland, 
2015; Giones and Brem, 2017; Figliozzi, Tucker and Polikakhina, 2018). On the business side, leading institutions predict a global UAV market to reach $\$ 21.8$ billion by 2027 from $\$ 8.68$ billion in 2019 at a compound annual growth rate (CAGR) of $14 \%$, including military and commercial applications (Meticulous Research, 2019). Even though military applications are expected to hold the largest share, the retail market is likely to grow with the fastest CAGR, mainly attributed to the increasing demand for UAVs for commercial activities such as delivering goods or mapping and monitoring. In addition, sectors with a large proportion in automatable operational processes are likely to benefit the most by adopting UAV technology for their routine tasks, leveraging lower costs and improved productivity (PWC, 2018). In their review, (Floreano and Wood, 2015) do not see significant technological or scientific barriers to advocate autonomous vehicles in research and commercial applications. To create impact, the technology needs to be mature enough, economically feasible and efficient. However, this only considers the (technical) innovation, which does not necessarily guarantee a successful diffusion or adoption.

Despite the prospects and market opportunities of UAV technologies, there is scant literature on the requirements and processes needed to support leveraging the technology innovation and to foster its wider adoption and diffusion in land administration contexts. Literature published so far dealt with the innovation process of UAVs with an emphasis on governance frameworks (Casiano Flores et al., 2020), institutional settings (Ho et al., 2019), or capacity frameworks (Tan, Flores and Crompvoets, 2021). By assessing the institutional dimension of innovation dynamics in Rwanda and Ethiopia, (Ho et al., 2019) conclude that UAVs, among other technologies, are still in the development phase determining a lack of financial resources as one of the significant barriers to scaled innovation. Interestingly, finances seem to be less of an issue for the scaling of UAV technology (Dijkstra et al., 2020). Instead, the authors identify the need for increased cross-sectoral partnerships and more national engagement and communication. Based on data from Rwanda, Casiano Flores et al., 2020 designed and evaluated a governance framework for the implementation of UAVs. It is reported that the governance context favours a top-down approach and that a strong focus on capacity development at local authorities could support an effective uptake for the implementation of UAV technology (Casiano Flores et al., 2020). Widening the scope to other innovative geospatial technologies (Bennett, Pickering and Sargent, 2019) identify political, socio-cultural and institutional settings within a system as the main determinants to 
steer the transformation of existing land administration processes. The studies presented thus far provide evidence that a holistic perspective, including technology and social and institutional systems, is fundamental to understanding the drivers and barriers to scale implementation of innovative technologies. However, in the context of UAVs, a comprehensive global analysis is absent.

This work elaborates on aspects determining the dynamics of scaled implementation of UAV technology in a global context to guide future decision-making processes. Frameworks from innovation theories and land administration are integrated to unveil the dynamics of the innovation process of UAVs on the one hand and its adoption by land administration processes on the other hand. To account for the progression of adoption, a temporal dimension is added to the framework. Empirical data stems from literature and interviews of UAV and land administration experts worldwide, reflecting on technology adoption rates among land administration stakeholders. The results of this chapter will provide a generalised knowledge base to derive information on processes and resources that create an environment in which UAV technology can thrive. Going beyond single-lens perspectives and adding a temporal aspect to the framework for efficient land administration, it is hoped that this chapter can guide decision-making processes on policies and implementation strategies concerning the application of UAVs in land administration.

The remainder of this chapter is structured as follows. Section 2 provides background information on the applied and combined innovation theory and land administration framework. Methods used for data collection and data analysis are outlined in Section 3. Results are presented in Section 4, laying out the assessment of experts concerning different stages of the uptake of UAV technology. The analysis and the subsequent evaluation on the importance of different pathways provide the baseline for Section 5, where progress and prospects for the scaled implementation of UAV technology in land administration are discussed. Finally, main conclusions and several future research directions are outlined.

\subsection{Background}

This research lies at the intersection of remote sensing, land administration, information systems, and innovation and draws on relevant frameworks and theories as outlined below. 


\subsubsection{Framework for Effective Land Administration (FELA)}

The framework for efficient land administration, in short FELA, is an overarching policy guide prepared by the Expert Group of Land Administration and Management of the United Nations Committee of Experts on Global Geospatial Information Management (UNGGIM), finding endorsement from UNGGIM in August 2020. With the aim to contribute to close the cadastral divide (Bennett et al., 2013), it guides countries that want to develop or reshape existing land administration and management systems (UNGGIM, 2020). Based on the concept of Integrated Geospatial Information Framework (IGIF) prepared by UNGGIM, FELA specifies nine strategic pathways to strive for effective land administration. Yet, it is not considered another concept in addition to existing approaches, such as fit-for-purpose land administration or pro-poor land administration, but rather as an allencompassing umbrella framework that accounts for and includes the existing knowledge base. Figure 5.1 depicts all nine pathways, including Legal and Policy; Finance; Data; Innovation; Standards; Partnerships; Capacity and Education; Advocacy and Awareness; and Governance, Institutions and Accountability. It is recognized and should be noted that even though nine pathways are defined, some of the focus areas are linked and necessarily overlap (UNGGIM, 2020).

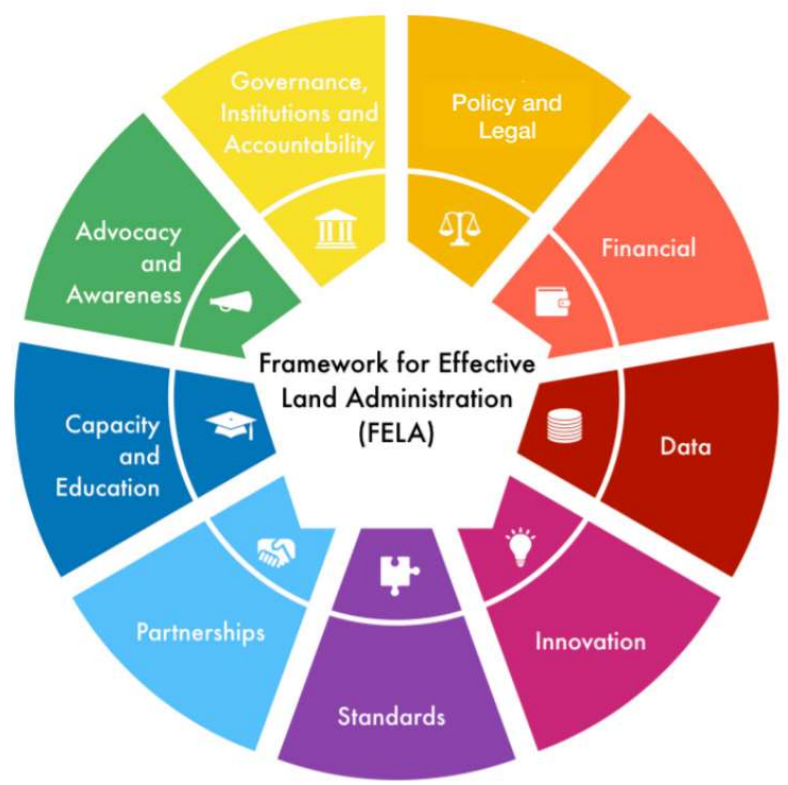

Figure 5.1: Nine Pathways of the Framework for Effective Land Administration (UNGGIM, 2020) 
With FELA's mission to support responsible innovation for effective land administration, this framework is helpful to analyse opportunities and hindrances for the scaled implementation of UAVs in land administration workflows. However, being only endorsed in 2020, translation of FELA into other languages, implementation tools, and methods remains ongoing at the time of writing. That said, the highlevel framework already finds application for gap assessments, if not project design work, in various country contexts. For example, one study describes a preliminary investigation in Nepal utilising FELA's categories to evaluate the role of UAVs and their impact on the land sector by using a traffic light assessment (Dijkstra et al., 2020). FELA was further used to investigate various land administration systems in the MENA region (Bennett et al., 2021). Although this worked well for describing the status-quo of land administration systems, this approach shows limitations concerning the progress of technology diffusion, which is highly likely to be different across various geographical regions. Some argue that especially countries of the Global South have a huge potential to accelerate technology adoption by skipping early and less efficient versions of innovation, i.e. technology leapfrogging (Davison et al., 2000). Thus, the next step involves selecting a suitable model that represents the innovation process over time.

\subsubsection{Technology innovation and diffusion models}

According to some researchers (e.g. Rogers, 1995), emerging technologies typically progress through different stages before they eventually reach majority and acceptance, fostering widespread adoption. In this context, the definition of key terms might be helpful to guide further discussion. (Rogers, 1995) defines innovation as an idea or object perceived as new and represents an alternative with new ways of solving problems. The same author outlines that diffusion can be understood as how innovation is communicated through specific channels over time. In contrast, the rate of adoption describes the relative speed at which entities of a social system adopt an innovation (Rogers, 1995).

Several models evolved during the past decades to predict the fundamental dynamics of diffusion and adoption of emerging technologies. The performance S-curve (Foster, 1986), the Adoption Curve (Rogers, 1995), and the Hype Cycle (Linden and Fenn, 2003) are ones widely used in existing works (Figure 5.2). All three curves indicate the evolution against time but consider different dependent variables on the $y$-axis. The time aspect is considered individually for 
each innovation, as not all technologies progress at the same speed. It is important to note that adopting innovative technologies is never the same and, in some cases, difficult to predict using such models.

The S-curve represents the general evolution process of the technology maturity over time, showing a sinusoidal line that is slightly increasing during the embryonic phase followed by a steep incline during the growth stage and again decreasing the slope when the technology reaches its maximum performance level. In contrast, the adoption curve model visualises the percentage of adoption, considered a normal frequency distribution and concludes that not all individuals adopt an innovation simultaneously. At a conceptual level, (Rogers, 1995) distinguished five different categories: Innovators $\left(2.5 \%{ }^{9}\right)$, Early Adopters (13.5\%), Early Majority (34\%), Late Majority (34\%), Laggards $(16 \%)$. Each category manifests different characteristics in terms of socioeconomic status, personality, and communication behaviour. Introduced by Gartner in 1995, the Hype Cycle depicts a curve showing expectations/visibility of emerging technologies based on the assumption that innovations will initially face overenthusiasm followed by disillusionment and eventually reach productivity. The curve merges the hype level curve (based on human attitudes) and the classical performance S-curve (Dedehayir and Steinert, 2016). Interestingly, most often, the invariant in the equation is people rather than technology, causing that most technologies conform to the Hype Cycle (Linden and Fenn, 2003).

Technology innovation and diffusion models have been increasingly used in the land domain, e.g. to understand the factors affecting the adoption of a land information system (Zeng and Cleon, 2018), to conclude on the opportunities of the blockchain (Kshetri, 2017), to discuss the expectations of UAV applications in agriculture (Freeman and Freeland, 2015), or the diffusion of GIS technology in the land and mapping agencies (Chan and Williamson, 1999), amongst others.

\footnotetext{
${ }^{9}$ Approximate percentage of individuals included in each category based on a normal frequency distribution.
} 


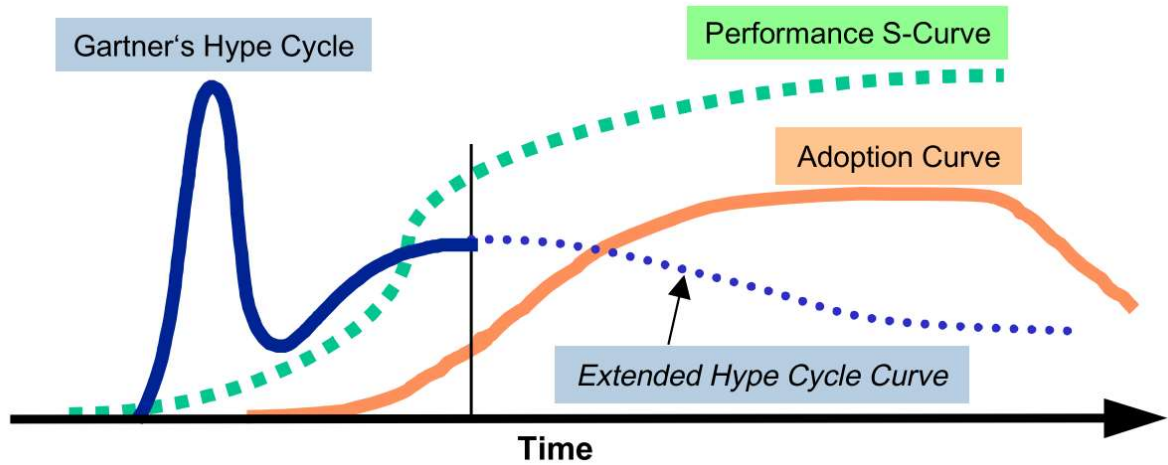

Figure 5.2: Prominent innovation curves plotted together. Source: (Linden and Fenn, 2003)

Although UAV technology reportedly reached a certain level of technology maturity, its use in the land administration domain is arguably not widespread (Ho et al., 2019; Stöcker, Ho, et al., 2019). Like other technologies, UAVs go through various stages before they are finally accepted and adopted by society. Since the Hype Cycle reflects on this phase, it is well suited to understand different levels of expectations and visibility in this early yet critical stage (Freeman and Freeland, 2015).

\subsubsection{Gartner's Hype Cycle}

Each year Gartner Inc. publishes Hype Cycles for 100+ technologies to advise firms on market potential and the (predicted) promises of emerging technologies. Aside from innovative technologies, Gartner Inc.'s scope is extended to strategies, standards, management concepts, competencies, and capabilities (Gartner Inc., 2021). The Hype Cycle takes place at an early stage of the technology's life cycle. As depicted in Figure 5.3, the Hype Cycle model proposes five sequential phases: innovation trigger, the peak of inflated expectations, the trough of disillusionment, the slope of enlightenment, and the plateau of productivity.

(Linden and Fenn, 2003) suggest that the first rise in the hype is triggered by $R \& D$ and early investigations with substantial media coverage. The sharp peak of exaggerated expectations is often followed by disappointing early results, causing the hype to collapse into a trough suddenly. Early adoptions fail to meet performance expectations, and adverse media reporting accelerate the decline in expectations, resulting in the trough of disillusionment. After some time, early adopters, who continued to work with the innovative 
technologies, eventually started to gain benefits. Investments are needed to push technology performance. Adoption will slowly rise, causing the rate of expectations to recover slowly. Methodologies and best practices evolve, and the technology starts to be socialised. The Hype Curve ends at the plateau of productivity where technology is realistically valued and adoption exceeds the rate of $20-30 \%$ of the potential audience.

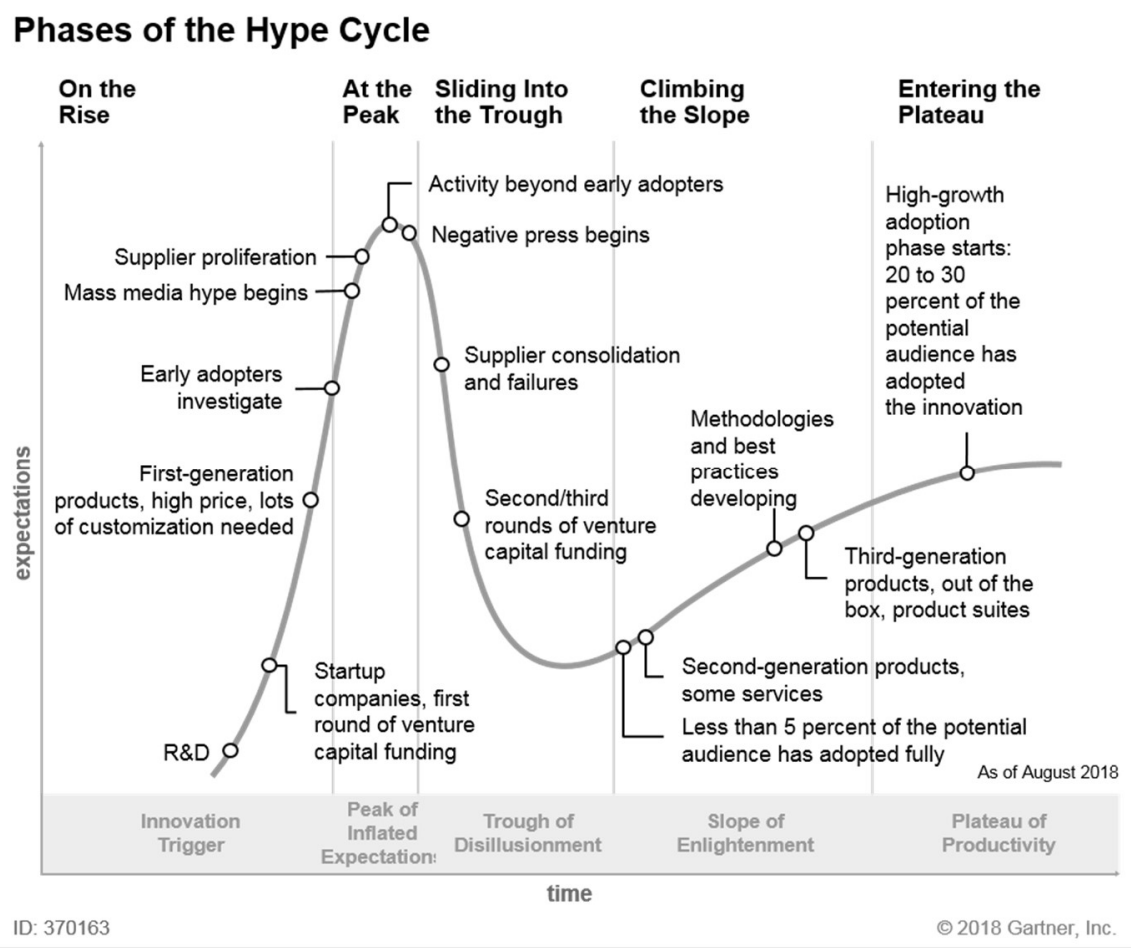

Figure 5.3: Scheme of the Hype Cycle, source: Gartner Inc. 2018

\subsection{Methods and Material}

Grounded in suitable theories to derive insights on the technology diffusion process in land administration, this research study followed a pragmatist philosophy (Tashakkori and Teddlie, 1998). As shown in Figure 5.4, data collection was guided by the underlying analytical framework, including the Hype Cycle model and FELA. Drawing on observations and experiences of experts in the land administration and UAV sector, results unveil a global perspective and best-practice examples. To this end, this research is more explorative than confirmative in nature and envisions how the use of UAV technology in land administration could reach the plateau of productivity. Data on 
the progression of UAV technology in land administration stems from a qualitative content analysis of interview transcripts from semistructured expert interviews. The analytical framework and methods for collecting and analysing data are further outlined below.

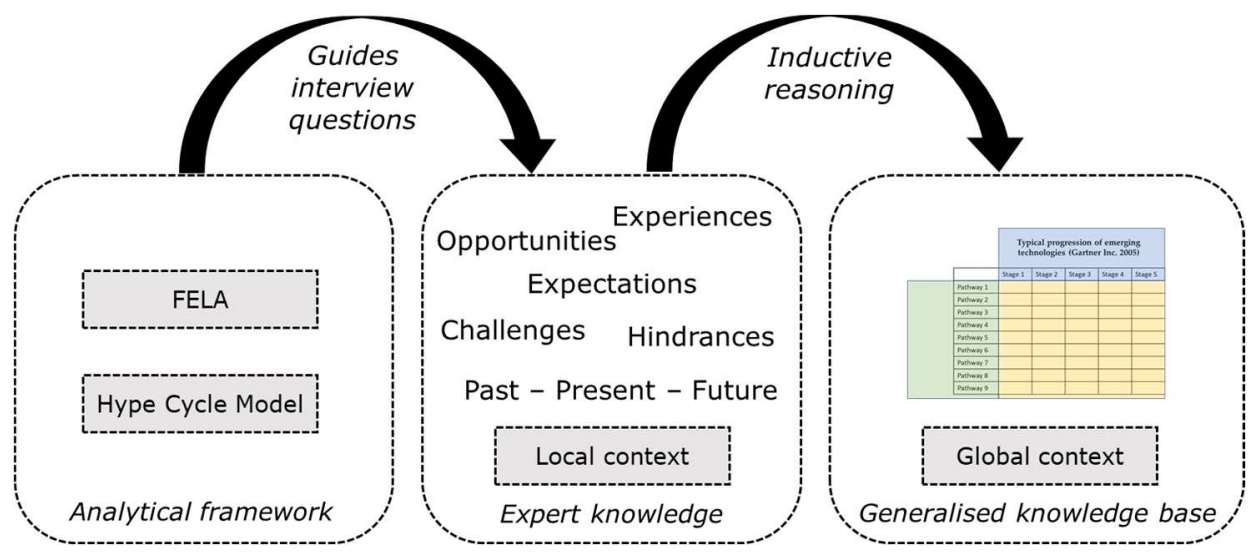

Figure 5.4: Scheme of the research approach

\subsubsection{Combining the Hype Cycle with FELA}

FELA provides an all-encompassing umbrella framework. Justified by its aim to support responsible innovation for effective land administration, this study utilizes FELA to set the context in which the diffusion of UAV technology occurs. The strategic pathways guide the analysis aiming to derive insights on how to embrace innovation and institutionalise UAV technology in land administration processes. However, FELA does not account for the temporal aspect of the innovation diffusion, which, as indicated above, revealed to be essential to understand the progression of emerging technologies. Thus, the Hype Cycle and FELA are fused with FELA to determine nine pathways that need to be considered for implementing effective land administration systems and five phases of the Hype Cycle, which add the temporal dimension of the emergence of UAV technology. This results in a two-dimensional matrix with 45 cells (Figure 5.5 ). 

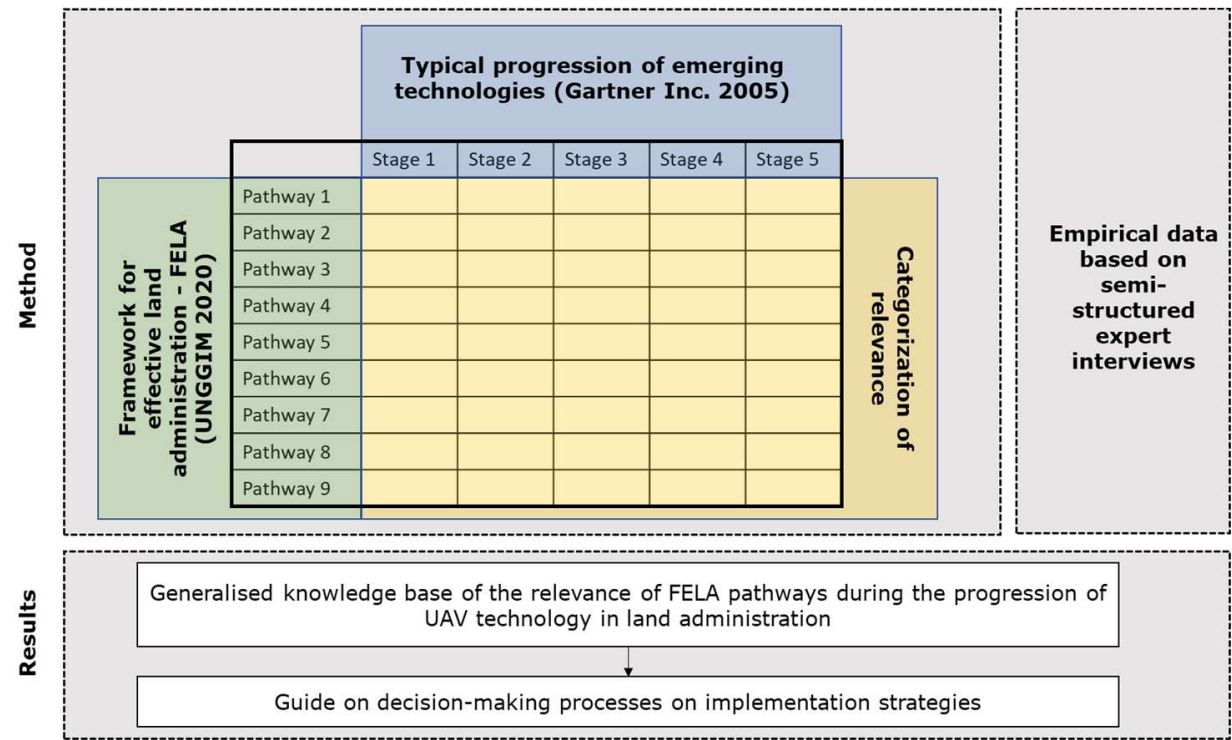

Figure 5.5: Scheme of the methodological approach and expected results. Empirical data is used to determine the importance of FELA pathways at different stages of the progression of UAV technology in land administration

As outlined by (UNGGIM, 2020), all FELA pathways need equal attention. At the same time, finding sufficient resources for innovation in land administration systems are an often reported challenge. By adding a temporal dimension to FELA, we can differentiate the importance of each pathway during the various stages. For this assessment, a three-level rating scale was developed to reflect on the relative importance of a FELA pathway at a given time: high importance, medium importance, low importance. High importance means that a pathway was reported to play a crucial role in advancing UAV technology as an innovation in land administration. If this aspect is not being addressed, the advancement of UAV technology might slow down or not progress any further. Medium importance implies that a pathway needs attention but is less essential for the progression at a given time. Low importance indicates that a pathway plays a minor role and is not explicitly crucial. If this pathway is not being addressed, it will only have a marginal impact on the advancement of UAVs in the land administration sector. It is essential to note that the ranking is relative and not meant to be quantified across different pathways. It cannot be translated into similar resources, be they financial and human, that need to be put in place for two or more pathways. The authors intentionally kept the scale to three categories to provide a simple relative assessment comparable to the works of (Casiano Flores et al., 2020; Tan, Flores and Crompvoets, 2021). Overall, it is hoped 
that the nuances can support policy and decision-making processes as they explicitly show which aspects to focus on most at a given time.

\subsubsection{Data sources and analysis}

Qualitative empirical data is used to derive insights on the diffusion process of UAVs in the domain of land administration which provides the basis for the three-level scale assessment of the combined Hype Cycle - FELA matrix. A linear sequential approach guided the data collection and analysis phase. From November 2020 to February 2021, the authors conducted nine semi-structured interviews via Zoom with national and international experts considered knowledgeable about the topic being investigated. The criteria for selecting experts were the documented engagement in a project that used UAVs for land administration purposes, emphasising technology implementation in 'real' practical projects. Data collection was designed to be crosssectional and included nine experts from the public sector (3), donor organisations (1), not-for-profit organisations (3), and the private sector (2). In general, the expertise of interviewees covered the following country contexts: Bulgaria, Germany, Ghana, Kenya, Korea, Kosovo, Indonesia, Namibia, Nepal, Peru, the Philippines, Rwanda, Senegal, Switzerland, Tanzania, and Vietnam. After some open-ended questions about the involvement in UAV-related projects, more targeted questions followed, covering aspects of all FELA pathways. Here, the use of UAVs in land administration only referred to the generation of base data for subsequent boundary delineation and adjudication. The interviewees were further asked about characteristics of an environment that would allow increased uptake of UAV technology. Another question sought to understand the main hindrance factors for scaled implementation, including reflecting past, present and potential future developments. Overall, the interviews took between one to two hours and were recorded after obtaining informed consent.

Data analysis followed the steps of a directed qualitative content analysis of interview transcripts (Hsieh and Shannon, 2005). Key concepts reflected the FELA pathways were used as coding categories (Policy and Legal; Financial; Data; Innovation; Standards; Partnerships; Capacity and Education; Advocacy and Awareness; Governance, Institutions and Accountability). One of the challenges of this method is the inherent bias of the qualitative interpretation and the risk that the researcher finds more data that is supportive rather than non-supportive of a theory. However, this risk is mitigated as the subject of this analysis is not whether a particular view - in this case, 
the Hype Cycle - is validated but how the respondent reflects on different FELA pathways at a specific time. By following this approach, the authors attempt to maximise the objectivity of the analysis accounting for increased validity and reliability (Potter and LevineDonnerstein, 1999).

\subsection{Results}

The results of this research study are twofold. First, the Hype Cycle curve is analysed based on reflections of the interviewees towards the emergence of UAV technology in land administration. Findings of the past progression, current status and future developments are reported. The second part elaborates on the combined FELA-Hype Cycle model and assesses the importance of FELA pathways during the Hype Cycle.

\subsubsection{Hype Cycle Analysis}

Most land administration experts could relate to various stages and name factors that negatively or positively contribute to technology diffusion in the land administration domain. Undeniably, it requires generalisation to look at this process globally, and various geographical contexts progress at a different speed. Some stages might be more influential than others as economic, governmental, or socio-cultural factors can considerably alter the advancement of technology adoption (Freeman and Freeland, 2015). However, overall, a similar pattern could be observed.

\section{Innovation trigger}

The use of UAVs for land administration was triggered by research and development projects between 2013-2017, mainly led by research institutions or donor organisations and presented at international conferences (Barnes et al., 2014; Kelm et al., 2014; Barnes and Volkmann, 2015; Mumbone et al., 2015; Meha et al., 2016; Koeva et al., 2017). The promotion of technology was heavily surrounded by other alternative concepts promoting aerial images instead of classical field surveys (Lemmen et al., 2015; Enemark et al., 2016). Since UAV technology has not been placed in concurrent land administration processes or organisational contexts, research mainly focused on the development and prototype stage.

As reported by the interviewees, the first pilot projects were initiated to collect aerial data to show the advantages of high-resolution imagery. At this time, most UAV flights did not require extensive flight permission procedures, and study areas covered only a few hectares 
(Manyoky et al., 2012; e.g. Rijsdijk et al., 2013; Devriendt and Bonne, 2014). However, through media reports in professional magazines and conference presentations, the idea of using UAV technology in land administration started to diffuse among the expert community. Through the spread of its potential impact (Kelm, 2014; Rubinov et al., 2015), expectations and visibility of UAV technology have begun to rise towards the second stage of the Hype Cycle.

\section{The peak of inflated expectations}

In contrast to the innovation trigger, the time of the peak of inflated expectations is more challenging to define and differs substantially from country to country. Expectations culminate in a peak, and benefits were reported to relate mainly to $\mathrm{cm}$-level accuracy (often rooted in a misconception between accuracy and resolution), unlimited access to technology and widespread application of it. Although technology-wise, these expectations can be satisfied, at least largely; legislative, administrative and institutional settings were not yet ready to allow the full exploitation of UAV technology in land administration at that stage. Throughout the interviews, several aspects were identified concerning problems of implementing UAV technology and contribute to a decline in expectations. First and foremost, pre-mature and prescriptive UAV regulations were mentioned, making it almost impossible for early adopters to enter the market. With the growing UAV industry, more and more concerns arose around public safety and data protection. As a response, countries enacted regulations determining procedures to receive flight approval (Stöcker, Bennett, et al., 2017). If UAV regulations are not in place, UAVs are criminalised by default and service providers cannot take the risk of adopting this technology and face challenges in finding capital, insurance and clients. However, most experts look to the future with optimism and see a trend towards riskbased or triage-type UAV regulations. The EU regulations are a prominent example of harmonized UAV regulatory frameworks (European Parliament, 2018; European Commission, 2019).

Second, some interviewees reported on UAV-based mapping services that did not consider accuracy requirements. For most applications, information on survey accuracy and data quality are pivotal for further land administration tasks. However, the complexity of ground truth measurements and the relation between flight configurations and the quality of data output (cf. Stöcker et al., 2020) is often underestimated, if at all considered. Particularly in the global South, the ease of flying a UAV by just pressing a few buttons and observing the automatic flight mission creates incentives to adopt the technology 
by a layperson, at the expense of understanding requirements towards data processing and analysis. Several projects also witnessed technological failures related to the communication link between the ground control station and the UAV or issues with the autopilot, a somewhat typical reality of first-generation products available on the market (Linden and Fenn, 2003). Some experts mentioned that, as a consequence, actors in the land administration profession slowly appeared to lose their interest in UAV related data products. Several respondents reported on such bad experiences with UAV technology which spread faster than success stories and led to a bad reputation.

\section{Trough of disillusionment}

However, amid the disillusionment and scepticism of stakeholders that feared disruption, some UAV advocates are still trialling and piloting to unveil the realistic potential and show clear limitations of UAV technology for land administration. To realise these pilots, it has been said that UAV regulations need to be appropriate ${ }^{10}$; otherwise, entrepreneurial efforts are halted from the beginning. Workshops by relevant stakeholders, including civil aviation authorities, government agencies, and private sector companies, were reported to support institutionalising adequate UAV-based workflows in a given country context. Ideally, this dialogue unveils the values UAV technology can add to concurrent surveying practices side-by-side of classical aerial photography and ground surveying methods.

\section{Slope of enlightenment}

Cross-sectional advocacy can ultimately pave the way towards policies and standards accommodating and legitimising the use of UAVs in existing land administration procedures. Real-world experiences by a growing number of actors adopting UAV technology can contribute towards a holistic understanding of the technology's applicability, benefits and risks signifying the beginning of the slope of enlightenment (Linden and Fenn, 2003). Backed by political will and a clear understanding of the value added by the technology, organically created demand for UAV-based products is likely to push the market to grow. Several interviewees shared that if the value that UAV technology can add to existing procedures is understood, there will be money for it. This is considered a crucial aspect during this phase

${ }^{10}$ Appropriate UAV regulations were characterized by three conditions: riskbased, affordable, and timely. 
because what counts in the end is profitability. The interviewees further outlined that only if the implementation of UAV technology is profitable actors can become self-sustainable and advance from donor-funded projects.

\section{Plateau of productivity}

To reach the plateau of productivity, which is characterised by a mainstream adoption (approx. $20-30 \%$ of the target audience) and a complete acceptance of the innovative technology, substantial capacity-building efforts, both in UAV piloting and UAV data processing skills alike, are required. Almost all interviewees raised data literacy and data processing capabilities as a condition for increased technology adoption.

Overall, each stage's characteristics and the perceived conditions to proceed through the Hype Cycle argue for an interplay of top-down and bottom-up strategies, as summarised in Figure 5.6. R\&D efforts in the first stage by research institutions and donor organisations and piloting by public and private institutions in the third stage are clear bottomup initiatives trying to advance and advocate UAV technology in the land administration sector. However, the content analysis suggests that if top-down decisions on UAV regulations, land policies, and standards are not in place or in favour of allowing and accelerating the widespread use of UAVs, technology diffusion and adoption is likely to slow down or even stall as indicated by the red circles in Figure 5.6.
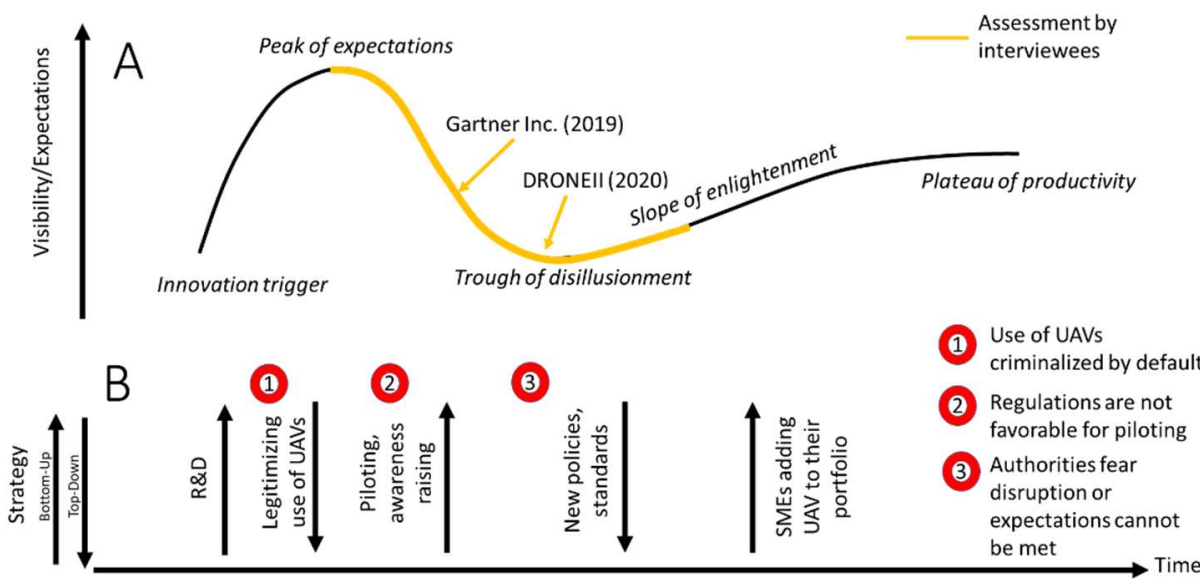

Figure 5.6: Reflections on the Hype Cycle of Gartner Inc. A: Assessment of experts on the status of implementing UAV technology in land administration processes. B: Strategies and forces influencing the innovation process, hindrances indicated in red circles 
As already mentioned above, this model is highly generalised, and various countries are likely to proceed at different speeds through this development. However, among the contexts covered during the interviews, a significant difference in innovation diffusion becomes apparent. A few interviewees indicated that they see the county where they worked just at the peak of inflated expectations. In contrast, the majority saw respective countries already sliding into the trough of disillusionment. More economically developed countries, in particular, were assessed to be on the slope of enlightenment. Besides empirical evidence sourcing from expert interviews, various reports have already been published about the status of UAV technology concerning the Hype Cycle. Although they do not focus on land administration applications, this assessment indicates the global evaluation of UAV technology. First and foremost, Gartner Inc. themselves published an extensive report summarising that commercial UAVs are currently sliding into the trough of disillusionment and seem to reach the plateau of productivity only in 5-10 years from now (Gartner Inc., 2019). Opposed to this, Drone Industry Insights (DRONEII) concludes in its yearly report Drone Industry Barometer that the UAV industry is already in the trough of disillusionment (DRONEII, 2019) - a phase to prove the concept with significant pressure to offer viable products.

\subsubsection{The relevance of FELA pathways during the progression of UAV technology in land administration}

The importance of the nine pathways of FELA was assessed individually for each of the five stages of the Hype Cycle. This results in a matrix with 45 positions (Figure 5.7). Whereas the assessment of the first stages is based on real-world experiences and derived best practices, the assessment of the last stage (the plateau of productivity) is based on the future predictions of the interviewed experts. Overall, each focus area shows a slightly different succession of its relevance, moving up and down over time. Some, like innovation obviously, seem to be key on an early stage and reduce in importance later. Some others are rated with the same importance at the beginning, and the end but have gone up (like legal) or down (like finance) in the middle steps. Standards and capacity appear most relevant towards the end of the Hype Cycle once more actors are adopting UAV technology. Almost all pathways, except for innovation, could be equally relevant at the slope of enlightenment, the most crucial phase when it comes to the commercial launch of an innovation. The peculiarities of each of the nine FELA pathways during the five stages of the Hype Cycle are described below. It should be noted that the scope of each pathway 
follows its definition in the Framework for Effective Land Administration (UNGGIM, 2020).

\begin{tabular}{|c|c|c|c|c|c|}
\hline & $\begin{array}{l}\text { Innovation } \\
\text { trigger }\end{array}$ & $\begin{array}{l}\text { Peak of inflated } \\
\text { expectations }\end{array}$ & $\begin{array}{c}\text { Trough of } \\
\text { disillusionment }\end{array}$ & $\begin{array}{c}\text { Slope of } \\
\text { enlightenment }\end{array}$ & $\begin{array}{l}\text { Plateau of } \\
\text { productivity }\end{array}$ \\
\hline $\begin{array}{l}\text { Governance, } \\
\text { Illstivions ond } \\
\text { Accountobility }\end{array}$ & High & High & High & High & High \\
\hline Legal ond Policy & Medium & High & High & High & Medium \\
\hline Finonce & High & Medium & Medium & High & High \\
\hline Dota & High & High & High & High & High \\
\hline Innovation & High & High & High & Medium & Low \\
\hline Stondards & Low & Low & Medium & High & High \\
\hline Portnerships & Medium & Medium & High & High & Medium \\
\hline 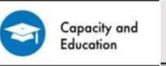 & Low & Low & Medium & High & High \\
\hline (3) Advocacy and & Medium & Medium & High & High & High \\
\hline
\end{tabular}

Figure 5.7: Combined matrix of FELA and the Hype Cycle with the assessment of the importance in each cell (High-Medium-Low).

\section{Governance, Institutions, and Accountability}

The governance, institutions, and accountability pathway is deemed a cross-cutting issue and fundamental throughout the innovation diffusion process. However, emphasis is put on different aspects at different times. Key actors named during the interviews refer to land administration agencies, civil aviation authorities, donor organisations, private surveying companies, research institutes, UAV consulting companies, grassroots initiatives and UAV vendors. During the innovation trigger phase, the primary responsibility lies with researchoriented institutions (i.e. both R\&D organisations or start-ups with an R\&D mindset such as Micro Aerial Projects ${ }^{11}$ ). Afterwards, an incremental process is seen as necessary to adequately support UAV technology/data governance in centralised cadastral agencies, which cannot be achieved without political will and adequate support from administrative instructions. In line with FELA, this can only be realised through strong leadership, tech-advocates and agents of change (public or private), which become particularly important during the trough of disillusionment and the slope of enlightenment, as reported in Ghana and Tanzania. But not only governmental institutions impose

${ }^{11}$ https://microaerialprojects.com (accessed on 13.07.2021) 
particular challenges to the adoption of innovative technology. For example, it was often mentioned that the surveying profession is "a tough nut to crack", with a lot of vested interest, uncertainties and worries around accuracy.

Experts mentioned two main opportunities for UAV data to positively impact existing governance and institutional structures and contribute to the FELA goals. First, due to its high spatial and temporal resolution, UAV data can significantly increase the transparency of land administration processes. For example, instead of lines and numbers, UAV data can enrich cadastral maps by adding a detailed base map that allows civil society to participate in the surveying process and substantially improves accessibility, particularly for the poor and vulnerable. Secondly, at the plateau of productivity, UAV data creation and sharing mechanism can break down the silos between land administration and land management authorities and ultimately strengthen multi-sectoral collaboration.

\section{Legal and Policy}

Except for the first and last stages of the Hype Cycle, which are assigned with medium importance, Legal and Policy are judged to be highly important when adopting UAV technology throughout. In this aspect, our assessment includes the legal frameworks surrounding land policies (including land administration) and UAV-specific flight regulations and, thus, extends the scope of FELA for this focus area. During the innovation trigger stage, the relevance was judged to be medium. With targeted R\&D projects, donor organisations and research institutions are encouraged to influence policymaking from the very beginning. At the following stages, adequate UAV regulations are considered one of the most necessary pre-conditions for scaled implementation in the land administration sector. All interviewees agree that a risk-based approach is the most favourable option for regulating UAV missions as prescriptive regulations impose substantial entry barriers for innovators and early adopters, as reported from Kenya. In addition, it has to be ensured that UAV regulations are managed by trained staff and that administrative, operational and technical requirements to obtain flight permissions are appropriately communicated to the public. This was experienced in Germany, but not so in Rwanda. Next, and equally important, UAV technology needs to be adopted in land policies to collect spatial data on land. Only if the use and application of UAV technology are legitimised, a market can start to develop. Once the legal foundation is laid with enabling UAV 
regulations and land policies, the relevance of the focus area Legal and Policy is expected to decrease at the slope of productivity slightly.

\section{Finance}

Besides the fact that all experts characterised UAV technology as a lowcost alternative to field surveying and its prominent contribution towards a more cost-efficient land administration system, the pathway Finance plays a crucial role during the innovation and adoption phase. Almost all interviewees mentioned a lack of finance as one of the main impediments to scaled implementation. Particularly expenses related to maintenance, import taxes and fees. Typically, R\&D activities during the innovation trigger phase are hardly paying off initially and need substantial government investments. Thus, they are rated with high importance. Subsequent stages involve piloting activities financed by donor organisations or venture capital funds of hardware or software companies. Only once expectations are starting to grow again and first adopters implement UAV technology for land administration workflows, the question of sustainable business models arises - a requirement that is also acknowledged in FELA. Without exception, all interviewed experts reported that the projects for land administration purposes alone could not cover all direct and indirect expenses related to hardware, software, maintenance and staff rates. It was further stated that a substantial amount of time is needed to be spent on stakeholder engagement - a service that is typically not paid. Consequently, private or public institutions offering "drones as a service" (DaaS) serve the surveying and mapping market and execute orders related to promotion, videography, monitoring, or inspections, among others, as reported from Bulgaria and Germany. However, international experts predict that after extensive awareness-raising campaigns and further proceeding through the innovation process, at least at the slope of enlightenment, the added value becomes more tangible. Once this is the case, service providers are expected to gain more revenue from mapping and surveying jobs.

\section{Data}

When data is the product of innovation, its quality is crucial for its uptake. Throughout all stages of the Hype Cycle, the pathway Data is perceived to be of high importance. Experts reported that the data product triggered excitement at the early stages and supported market development later on. High-resolution aerial photography, digital elevation models, or even 3D point clouds unveil details that were not visible before and allow to map cadastral boundaries on base maps that 
are more meaningful than satellite or aerial images. Guidelines and best-practice examples need to be developed to ensure that the data meets the requirement set by the land policy, an aspect where standards are becoming equally important. All experts discussed the concept of accuracy and highlighted the opportunities to serve a range of different technical specifications offered by various UAV sensors or ground control strategies.

Moreover, it was mentioned that UAV technology could empower local stakeholders to collect and own data by themselves by enabling a decentralised data collection strategy that is independent of the central government, as exemplified in Ghana, Tanzania, or Indonesia. Highresolution images were perceived as a game-changer that opened the discussion about land rights to a much wider audience, including marginalized groups and illiterate people. With the opportunity for high spatial and temporal resolution, UAV data can significantly contribute towards the FELA goals calling for reliable data and service quality. Aside from data quality, privacy and data protection concerns need to be accounted for and adopted from global ethical standards pertaining to locational UAV data.

\section{Innovation}

The relevance of the pathway Innovation is assessed to be high at the beginning stages and decreases towards the plateau of productivity. Following FELA, most interviewees reflected on technology push and societal pull as driving factors for innovation. In this aspect, the interviewees particularly mentioned the opportunities for process improvement and advancements concerning various sensors that can be deployed at the UAV (LIDAR, Radar, multispectral cameras). By adding UAV technology to the toolbox, the surveyor has yet an additional complementary tool to ensure that his services best fit with the administrative requirements set by the contracting authority. Societal pull calls on the ability of UAV technology to contribute to alternative land administration concepts. In particular, interviewees mentioned the opportunity to bring the data back to the community, or even provide it immediately, and foster a decentralised land administration system.

The growing number of UAV-related sessions during world-leading land conferences such as the Land and Poverty Conference or the FIG Conferences proves the increasing importance of innovative UAVbased solutions throughout the last years. Furthermore, recurring expos and events such as the Africa Drone Forum 2020 and the Lake 
Kivu Challenge, which started in 2020, help mature the Global South's innovation culture.

\section{Standards}

This focus area is a cross-cutting issue and is closely related to Data and Legal and Policy. All experts agreed on the importance of adding UAV technology to existing (surveying) standards to accelerate technology uptake by legitimising its application. Thus, the relevance of the pathway Standards increases once the public and private sectors are starting to adopt UAV technology towards the slope of enlightenment. Interviewees referred to necessary national standards on processing aerial photography, map production standards, and survey accuracy standards. For instance, although UAVs have been widely used in Indonesia for several years, they have only recently been accepted by the spatial agency to collect data for base maps for further land administration processes. Despite the General Data Protection Regulation safeguarding personal data at the European level, no international standard was reported on dealing with the use of UAV data for land administration. Perhaps, this could be a future area for international professional organisations such as the FIG.

\section{Partnerships}

As the implementation of UAV technology cuts through multiple actors, the pathway Partnerships were perceived to be critical throughout the process. However, particularly during the stages where pilots are set up, and the market is expected to develop, the importance of partnerships was rated as high, as demonstrated in Tanzania. At the beginning of the innovation process, partnerships with international organisations and academia facilitating R\&D and pilot projects are deemed essential to convey the message: "There is some new technology out there. Here are the options of how to use it here is how to use it safely here are cost implications.". However, later in the process, Public-Private-Partnerships gain importance to establish an enabling environment where UAVs are offered as a service. Moreover, UAV technology provides a huge opportunity to increase nongovernmental actors' responsibility and play an active role in current data collection efforts, as reported from Ghana and Namibia. Additionally, regulating bodies such as civil aviation authorities should be engaged with from the beginning. Nepal has shown that this partnership can be precious for co-creating efficient flight authorisation procedures (Nepal Flying Labs, 2020). 


\section{Capacity and Education}

Capacity development is crucial for creating local innovators and entrepreneurs. Similar to the focus area of Standards, the relevance of Capacity and Education increases over time. For the use of UAV technology in land administration processes, capacity development comprises various aspects. For example, most experts reported that UAV piloting is not an issue due to its simplicity and improved automation. The flying capabilities are also covered in national UAV legislation, which typically mandates basic practical and theoretical pilot training. This contrasts with data processing and data literacy, two aspects perceived to be among the main hindrances for scaled implementation. However, most countries that were covered during the interviews recently embedded UAV-related educational courses in existing GIS or surveying curricula and expect to produce young professionals (e.g. in Bulgaria, Kenya, Kosovo, Nepal). Training courses by established international academic institutions or sophisticated online workshops are an additional opportunity to enhance local knowledge. A key challenge was identified concerning data literacy, understanding the value of the data and its contribution to land administration processes.

\section{Advocacy and Awareness}

Similar to the statement in FELA, interviewees showed consensus that the application of UAV technology in land administration cannot flourish without the support and acceptance of stakeholders and society. Once pilot studies are in place, and expectations are still declining, awareness-raising campaigns and advocacy become essential and the importance of the pathway Advocacy and Awareness increases. In this context, two main themes were identified as most crucial. First, awareness-raising campaigns should not focus on the technology but on the value UAV data can add to solve land administration challenges. One of the experts reports that "The problem that I see with drones is like everybody gets super excited and then everybody wants to do it, but nobody asked themselves like what was the end goal here? What are you actually trying to achieve? What is the outcome, not the output?". Second, sensitisation and advocacy with the organised profession play an essential role. The majority of interviewees reported the private surveyors or government staff are afraid that somebody will take their jobs or become redundant. If this is not acknowledged during the innovation process, the actors expected to adopt will renounce UAV technology. 


\subsection{Discussion}

The findings demand reflection on two main aspects relevant in the current debate of technology adoption. First, institutional constraints and opportunities offered by UAV technology to embed change into existing land administration workflows are discussed. The second part elaborates on the applicability of the Hype Cycle and FELA as global frameworks and their relevance for national contexts.

In the past, land reforms and technology innovations were significant drivers of regime change (Jepsen et al., 2015). However, at the same time, institutions oppose this social force with stability and consistency. Thus the relationship between innovation and institutions is often connotated with tension (Ho et al., 2013). Reflecting on the findings, UAV technology offers various opportunities to incept change but is also challenged by rigorous institutional frameworks and vested interest. One central aspect concerns the organization of land administration activities. UAVs can facilitate local actors with a surveying tool to realise a meaningful engagement of communities and local citizens as an integral part of effective land administration systems. In this vein, the central government or other dominant organizations (e.g. donors) would no longer be the only data provider of high-resolution aerial data, and local communities or agencies can determine the data collection strategy according to their requirements. However, particularly in authoritative countries, government institutions fearing losing power and control can resist this decentralised approach.

Similarly, the increased transparency provided by UAV data related to land use and land management might be an undesired by-product hampering the scaled implementation, particularly in countries with corrupt political institutions. In most countries, the surveying profession is highly standardized, which can be both a challenge and an opportunity. Bounded by surveying regulations and standards that do not include UAV-based procedures, the organized profession has little incentive to deviate from business as usual. As outlined by (Hargadon and Douglas, 2001), entrepreneurs should strive to embed their ideas within the set of prevailing understandings that mark the institutional setting yet contrast their innovations to existing solutions. Best practices by entrepreneurs and technology advocates as well as a clear understanding of the cost and time savings of UAV-based workflows are expected to support the uptake of UAV technology as an additional surveying tool - side by side with traditional equipment such as GNSS, total stations or theodolites. As reported in the findings, a 
business model including "drone-as-a-service" seems to be the most likely option for early adopters. In addition, responsibility to incept change should also be carried by donor organizations supporting landrelated projects in the Global South. Western donors often contract companies from their own countries to get a job done without investing in domestic markets (Cheney, 2019). Instead, tenders should invigorate local partners to incentivize the development of new market segments at the geographical region of the beneficiary.

The Hype Cycle depicts a typical pattern that arises with each innovation showing typical progress through an evolution of overenthusiasm and disillusionment followed by eventual productivity. By assessing the importance of FELA pathways throughout the Hype Cycle, we depict a generalized pattern that evolved from best practices and expert opinions on future developments (i.e. towards the slope of enlightenment). Our findings do not specifically focus on the country level but rather model context-specific dynamics and requirements in the process to reach the plateau of productivity and ultimately foster increased adoption of UAVs in land administration. Following (Linden and Fenn, 2003), innovation profiles may be at different positions in different geographical regions. With UAV technology in general and its implementation in land administration contexts, the findings suggest that national policies and infrastructure fundamentally steer innovation dynamics. The firm reliance on infrastructure and regulations, two aspects that need time to evolve, means that UAVs are instead a longfuse technology showing a slower adoption (c.f. Linden and Fenn, 2003). Depending on these inherently different circumstances from one nation to another, some countries may lag behind, and others might be further ahead. Eventually, some countries might even skip early technology development stages. The latter is also referred to as technology leapfrogging (Davison et al., 2000). For UAV technology, this has been reported for the agricultural and medical sector highlighting examples from the Global South (Cheney, 2019; Radovic, 2019; Haula and Agbozo, 2020). However, it is beyond the scope of this investigation to infer on technology leapfrogging in the context of land administration.

Overall, our generalized assessment does not claim to be the ultimate guide to make UAVs succeed in the domain of land administration. Instead, with the nuanced importance of pathways over the process of technology emergence, FELA can be seen as a set of levers that can be utilized or calibrated differently, at different moments, to support innovation. 


\subsection{Conclusion}

UAVs are increasingly used to aid mapping endeavours worldwide. However, adoption and implementation in the land administration domain remain low. Embedded in the Hype Cycle and FELA concept, this study analysed the emergence of UAV technology in land administration. It assessed the importance of FELA elements from the initial phase of innovation trigger to the plateau of productivity. Nine experts could elaborate on 16 different country contexts. None of the experts reported that the plateau of productivity was reached already. The majority saw respective countries already sliding into or being at the trough of disillusionment. This presents a phase in which the innovation needs to overcome disappointing expectations, and a market is yet to develop, and adoption starts to rise.

Notwithstanding the importance of all FELA pathways throughout the innovation process, it can be concluded that scaling up the use of UAVs from a niche market to a widespread application in land administration contexts is led by alternating top-down and bottom-up dynamics. Enabling laws and policies and supporting governance, accountability and institutions are crucial to create such a UAV-friendly national ecosystem early on in the process of technology adoption and allay exaggerated expectations. Once this ecosystem has been made, market demand is expected to surge driven by partnerships, adapted standards, tech advocacy and awareness-raising campaigns highlighting the superiority of high-resolution data amongst other benefits of UAV technology. The generalized global perspective reported here sheds new light on the dynamics during the innovation process of UAV technology in land administration. The assessment of the importance of FELA pathways can be used as a baseline to direct strategic decisions concerning national policies and guidelines towards the scaled implementation of UAVs in land administration.

The scope of this study was limited to a generalized assessment of the emergence of UAV technology in land administration. Data from the expert interviews did not suffice to conclude country-specific developments or technology adoption rates. Thus, further research should be undertaken to study the impact of UAVs as disruptive technology on existing land administration workflows in a national context. The trajectory of countries that succeed towards the plateau of productivity and eventually adopt UAV technology for land administration could further be compared to the generalized model presented in this chapter. 
Chapter 6 -Summary of the Main Findings and Reflections 


\subsection{Introduction}

This thesis was motivated by the fact that a large share of the global population has no documented land rights. Insecure land rights can lead to conflicts, instability and the exclusion of vulnerable and marginalized groups. In this context, the documentation of land rights is an essential step to make progress towards a more peaceful and sustainable future of our planet and achieve the SDGs to build inclusive, resilient, and sustainable societies. New tools and approaches are needed to overcome the cadastral divide (Bennett et al., 2013). Amongst others, Unmanned Aerial Vehicles (UAVs) are emerging as a tool for alternative land tenure recording.

Even though UAV technology appears a promising technology, it was not clear to what extent it can address the global land administration challenge to increase the number of people enjoying tenure security. Thus, the main objective of this dissertation was to explore UAV-based data acquisition workflows as a tool for effective land administration. Chapter 1 sets the scene and introduces the main concepts and the methodological approach of this thesis. Furthermore, it outlines the problem statement and research objectives. Chapter 2 to chapter 5 embrace the research investigations embedded in the socio-technical assessment. As such, chapter two examines the nexus of land data, user needs and UAV imagery and analyses to what extent stakeholder needs can be met by UAV technology. Chapter 3 analyses past, present and future developments of UAV regulations which are considered a crucial requirement for pursuing UAV data acquisition workflows. In chapter 4, different aspects impacting the final quality of UAV-derived orthophotos as base maps for cadastral mapping are examined. The outcomes of chapters 2, 3 and 4 feed into chapter 5, integrating innovations theories and land administration frameworks to understand the dynamics of the implementation of UAV technology in land administration.

The following subsections are guided by the sub-objectives as outlined in the introduction:

1) To examine stakeholders' land information needs and understand how UAV technology can meet respective requirements.

2) To analyse the interface between UAV data acquisition workflows and stakeholders in terms of legal and regulatory frameworks. 
3) To assess different data quality measures of UAV-based orthophotos and recommend optimal UAV data acquisition workflows.

4) To understand the dynamics of the diffusion process of UAV technology as a tool for effective land administration.

\subsection{Conclusions per research objective}

\section{To examine stakeholders' land information needs and understand how UAV technology can meet respective requirements.}

The presented work in chapter two highlights the capabilities of UAV technology to match the needs of land professionals in Rwanda. A needs assessment enabled expressing a range of land information needs across multiple levels and stakeholder sectors. Next to the social study, three different UAVs were flown to test the quality of data and the possibilities of using this technology within the current institutional environment. A priority list of needs for cadastral and non-cadastral information and insights into operational challenges and data outputs are presented. Appendix 2 supplements the research evidence by reporting on a practical UAV-based community mapping activity. Results of the sound needs assessment demonstrate the prioritised demands of respective respondents. Although ranked differently, the need for high-resolution, up-to-date land information is consistently identified in the final lists of all group discussions. The results suggest that UAVs as a data acquisition device could most likely be adopted by national-level stakeholders or sub-national government stakeholders, which can be attributed to the system in Rwanda where the national government is the primary provider of geospatial data.

The examination also suggests four main aspects which may reveal challenges to UAV interventions to derive land information in the context of Rwanda: 1) the hilly terrain, 2) UAV regulations, 3) groundtruthing in urban areas, 4) data processing capacities at the national land administration authority. Next to these operational challenges, the integration of the needs assessment and the UAV test flights indicates that structural and capacity conditions currently undermine the vast potential of the UAV data acquisition method. Therefore, a key policy priority should be to implement country-specific capacity development and governance strategies. However, notwithstanding the outlined challenges, the results of this study show that UAV technology has the 
potential to make a significant contribution to delivering the base data for most of the prioritized land information needs in Rwanda.

\section{To analyse the interface between UAV data acquisition workflows and stakeholders in terms of legal and regulatory frameworks.}

This analysis was triggered by our own experiences in Rwanda, Kenya and Ethiopia and started when we faced a situation in which current legal frameworks that regulate UAVs presented significant barriers to our research plans. Based on these experiences, we were curious to understand if this situation is unique to our study sites or whether this situation is representative of global developments. Based on a research synthesis that included a thorough literature review and comparative analysis of 19 national regulatory frameworks, similarities and contrasting elements in various national UAV regulations and their implications for data acquisition activities were explored. In essence, regulations target the management of risks and minimization of perceived harms. Within the context of UAVs, the main harms are malfunction, mid-air collisions and consequential damages to people and property on the ground.

In essence, chapter three suggests that UAV regulations focus upon three key aspects: (1) targeting the regulated use of airspace by UAVs; (2) imposing operational limitations; and (3) tackling the administrative procedures of flight permissions, pilot licenses and data collection authorization. Since the early 2000s, countries have gradually established national legal frameworks for UAVs. Although all UAV regulations aim at one common goal-minimizing the risks for other airspace users and people and property on the ground-a distinct heterogeneity of national regulations exists. However, commonalities such as mandatory platform registration, obligatory insurance coverage and standard pilot licensing procedures indicate trends towards mature national UAV regulation. Desirable trends were seen in the coexistence of hard and soft regulations and the successful international dialogue that may eventually provide a legal framework for harmonized regulatory standards.

To assess different data quality measures of UAV-based orthophotos and recommend optimal UAV data acquisition workflows.

The fact that UAV-derived geospatial information supports decisionmaking processes involving people's land rights ultimately raises 
questions about data quality and accuracy. In this vein, this chapter investigated different flight configurations to give guidance for efficient and reliable UAV data acquisition. Imagery from six study areas across Europe and Africa and overall more than 100 scenes representing different flight configurations provide the basis for an integrated quality assessment including three main aspects: (1) the impact of land cover on the number of tie-points as an indication of how well bundle block adjustment can be performed, (2) the impact of the number of ground control points (GCPs) on the final geometric accuracy, and (3) the impact of different flight plans on the extractability of cadastral features. It was found that land use has a significant impact on the generation of tie-points. Image scenes characterized by a high percentage of vegetated areas, especially trees or forests, require image overlap settings of at least $80 \%$ to $90 \%$ to establish sufficient image correspondences.

Moreover, independent of the size of the study area, the error level of planimetric and vertical residuals remains steady after seven equally distributed GCPs, given at least $70 \%$ forward overlap and $70 \%$ side lap. As exemplified for concrete walls, the quality of reconstructed thin cadastral objects is highly variable to the flight configuration. A large image overlap and a cross-flight pattern have proven to enhance the reliability of the generated orthophoto as quantified by the increased accuracy and completeness of automatically delineated walls. In contrast, the delineation results of rooftops showed less sensitivity to the flight configuration. Moreover, even though the RMSE of checkpoint residuals as a commonly accepted error measure is within a range of few centimetres in all datasets, chapter four revealed large discrepancies in the accuracy and the completeness of automatically detected cadastral features. The results highlight that scene context, flight configuration, and GCP setup significantly impact the final data quality of resulting orthophotos and subsequent automatic extraction of relevant cadastral features.

\section{To understand the dynamics of the diffusion process of UAV technology as a tool for effective land administration.}

Despite the prospects and market opportunities in land administration, there is a gap between experimentation and widespread diffusion of UAV technology. In this work, the Framework for Effective Land Administration (FELA) and the Hype Cycle concept are integrated to understand the dynamics of the innovation process of UAVs for the land administration sector. Empirical data stems from literature and interviews of UAV and land administration experts worldwide. Most 
experts estimate UAV technology to be in a phase in which the innovation needs to overcome initial unmet expectations to foster market development and increased adoption. Notwithstanding the importance of all FELA pathways throughout the innovation process, it has been shown that the process of scaling up the use of UAVs from a niche market to a widespread application is led by alternating top-down and bottom-up dynamics. Enabling laws and policies in addition to supporting governance, accountability and institutions are crucial to create a UAV-friendly national ecosystem early on in the process of technology adoption and allay exaggerated expectations. Once this ecosystem has been made, market demand is expected to surge driven by partnerships, adapted standards, tech advocacy and awarenessraising campaigns highlighting the superiority of high-resolution data amongst other benefits of UAV technology.

\subsection{Conclusions on the main research objective}

\section{To explore UAV-based data acquisition workflows as a tool for responsible land administration.}

Two themes drive the conclusions on the main research objective. First, the results of the sub-objectives are put together to take a holistic perspective of UAV-based data acquisition workflows in land administration contexts. The second part concludes on the role of the main findings for advancing responsible land administration.

The integrated view on the socio-technical system, including stakeholders, land administration processes and UAV technology as system elements, allows identifying interactions and positive and negative dependencies. Guided by the results presented earlier, we can infer that the researched nexuses of this thesis, namely land data needs (chapter 2), UAV regulations (chapter 3 ), data quality (chapter 4) and technology diffusion (chapter 5), are closely interrelated. Whereas the aspects of data quality and regulations both influence but are also impacted by other elements, land data needs were found to have a critical role in the system and predominantly affect other elements. Underpinned by contemporary land administration approaches (Zevenbergen et al., 2013; Enemark et al., 2014), land data needs are the central starting point for any land tool intervention. In our socio-technical system, land data needs determine data quality by creating the terms of reference to which UAV data must comply. To this end, chapter 4 endorses various flight configurations allowing to design flight missions to meet the agreed requirements. On the other 
hand, land data needs may challenge existing - mostly pre-mature UAV regulations calling for mature risk-based (inter)national laws that lower the entry barrier for UAV service providers to establish a demand-driven UAV mapping market. Looking further into regulations, the execution of optimal flight configurations required to meet data quality needs may be constrained depending on administrative requirements or operational limitations promulgated at the national level.

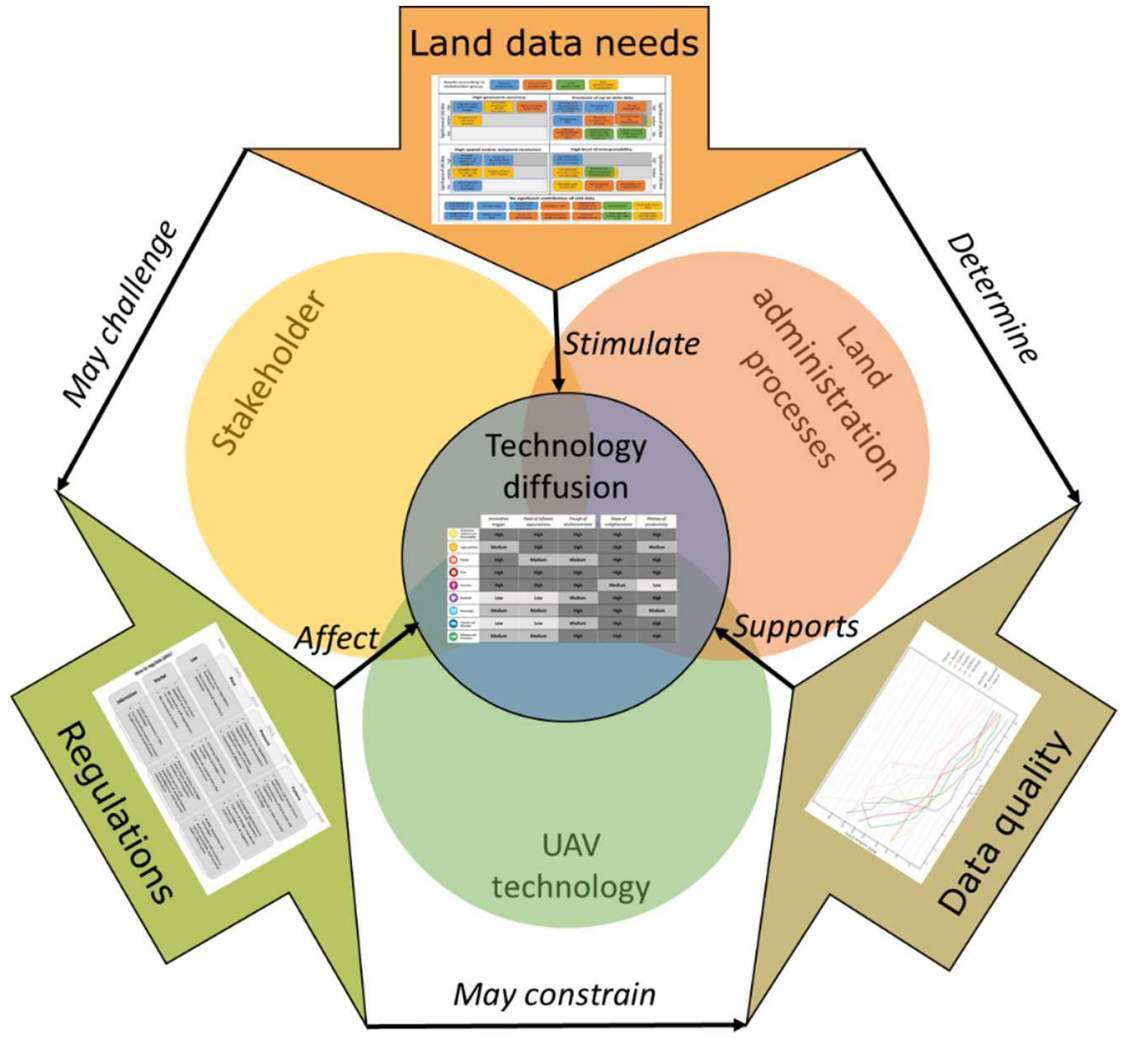

Figure 6.1: Main conclusions on the dependencies of the nexuses in the socio-technical system including land data needs (chapter 2), UAV regulations (chapter 3), data quality (chapter 4), and technology diffusion (chapter 5). Embedded figures are further explained in the corresponding chapter.

In our framework, the diffusion of UAV technology in land administration takes a central place. It is impacted by requirements for land data, regulatory frameworks, and data quality, as investigated in this thesis. Firstly, the superior quality of UAV data is a key driver throughout the diffusion process. In contrast, regulations may 
positively or negatively affect the emergence of UAV technology, as appropriate UAV legislation presents a vital pre-condition for technology uptake. Third and most importantly, land data needs stimulate the scaled implementation creating a demand for up-to-date high-resolution land data.

The second part of this conclusion infers on the role of the presented findings in advancing responsible land administration. This dissertation aligns well with the endorsed practices regarding land (Zevenbergen, De Vries and Bennett, 2015) as the research takes a multi-stakeholder focus, uses insights from multiple disciplines, and is proactive, considers a global context and advocates for societal relevance and practicability. The multi-stakeholder direction is represented in chapter 2 and chapter 5, including the needs assessment among various governmental and non-governmental actors and the expert interviews with representatives from NGOs, practitioners and national governments. The connection of insights from technical assessments, social sciences, law, and innovation systems makes this dissertation's results truly cross-disciplinary and allows us to infer a holistic perspective. Ethical and societal aspects are mainly addressed in chapter 2 and chapter 3 , reflecting on societal demands, the interplay of law and technology, and the risks UAV technology may pose to civil society and how the risks are mitigated. Being embedded in the H2020 funded international research and innovation project its4land, parts of this thesis (especially chapter 2 and chapter 4 ) were exemplified in the East African context, including test flights, workshops, and mapping activities.

In contrast, chapter 3 and chapter 5 take a global perspective and elaborate on UAV regulations and the emergence of UAV technology on a global scale. Practicability was essential in chapter 4 that draws recommendations on optimal flight configurations. Societal relevance is inherent to the discussion on the emergence of UAV technology as an innovation in land administration, as presented in chapter 5. Overall, the findings of this thesis suggest that UAV technology is a highly flexible, widely available, easy-to-use and end-user responsive data acquisition tool that complements the range of mapping tools for land administration processes side by side with satellite data and GNSS surveying. Yet, regulatory constraints, missing awareness and weak governance support currently hamper exploiting the full potential in a large share of countries. 


\subsection{Implications of the results}

\subsubsection{Contributions to scientific research}

The most important contribution of this dissertation to the knowledge base refers to the holistic assessment of UAV technology in land administration. Previous works - which seldom exceed the scope of feasibility studies - mainly refer to data-driven and technological assets of the utilization of UAVs and do not consider societal, governance and institutional settings and needs of people and groups. To address this gap, this thesis aligns technology-push with societal-pull via a sociotechnical investigation considering technology, processes and people in a holistic framework.

Looking through a methodological lens, three aspects may advance the knowledge base. First, chapter 3 developed a novel methodological framework to analyse UAV regulations by assigning their content to six core dimensions:
1) Applicability
4) Administration procedures
2) Technical requirements
5) Human resource requirements
3) Operational limitations
6) Ethical constraints

This framework may help to enhance the comparability of existing UAV regulations even though institutional and organisational structures might be diverse.

The second aspect, which marks a contribution to the knowledge base, encompasses investigated discrepancies between the spatial accuracy and the completeness of automatically detected cadastral features, even though the RMSE of the orthophoto as a commonly accepted error measure is low. According to these data, we can infer that the flight configurations play a crucial role in achieving high data quality, particularly for cadastral features characterized by height differences and thin shapes, as exemplified in the case of concrete walls. Consequently, it should be emphasized that a context-driven error analysis as presented in this thesis is essential to assess the overall accuracy of UAV-based data products for different applications.

The third aspect addresses our investigation of the process of technology diffusion. Finding sufficient resources for innovation in land administration systems are an often reported challenge. Based on technology innovation and diffusion models, we added a temporal dimension to FELA, allowing us to differentiate the importance of each pathway during the Hype Cycle stages representing the sequence of a 
typical innovation. In turn, this itemization allows attributing the pathways as a set of levers that can be utilized or calibrated differently at different moments to support technology diffusion. Although this methodological approach was developed and exemplified with the subject of UAV technology, it may also be adapted to other emerging technologies in land administration contexts.

\subsubsection{Contributions to land administration and policy}

Current land administration approaches guided this work and the quest for innovative geospatial solutions to address the immense land administration challenge to map millions of undocumented land (Rahmatizadeh et al., 2018; Lengoiboni, Richter and Zevenbergen, 2019). In this regard, UAV technology as an innovation in land administration has been investigated in a socio-technical framework, including deep dives from various angles. This dissertation focused on the provision of base data which is essential for subsequent mapping campaigns to ascertain land tenure, land value, land use or land development. Chapters 2 and 4 showed that UAV technology is mature enough to contribute to the paradigm shift in land administration. Of course, it is not UAV technology alone that will bring tenure security. Still, it has been shown that UAV technology is a viable tool that may close the gap between highly accurate but time-consuming field campaigns and less detailed satellite imagery. It is found that UAV technology may mainly benefit bottom-up and grassroots organisations as it presents a means to collect spatial base data at the local level effectively.

The possible emergence and diffusion of UAVs in land administration are presented in chapter 5 . This chapter further assesses the importance of different pathways which may be used as a baseline to direct strategic decisions concerning national policies and guidelines towards the scaled implementation of UAVs in land administration. This assessment is based on the Framework of Effective Land Administration, an overarching policy guide prepared by the Expert Group of Land Administration and Management of the United Nations Committee of Experts on Global Geospatial Information Management. It thus relates to the most recent policy developments in land administration. At the bottom line, advocacy and awareness are particularly important at the current stage of innovation diffusion in which a demand-driven market is yet expected to develop. During this research, findings were continuously discussed and disseminated on various occasions, including national, regional and international land- 
related conferences and workshops to promote the opportunities and limitations of UAV technology in land administration contexts.

As with any new technology, UAVs can be used for good or bad. However, by adhering to fundamental human rights, imposed regulations and a strong materialisation of responsible land administration, the use of UAVs as a practical tool in land administration may contribute toward shared prosperity.

\subsubsection{Contributions to UAV practice and policy}

This work has shown that data quality significantly changes depending on the flight configurations presenting risks and opportunities simultaneously. The risk is that UAVs are used as off-the-shelf products with little knowledge of photogrammetric principles and options to customize flight configurations. The opportunities show that different flight configurations and ground-truthing measures offer a wide range of options to tailor the data collection task to financial, personnel and time capacities and optimally align it to customer needs and requirements in the land sector. The recommendations outlined in chapter 4 encompass basic principles that may be considered by anyone conducting UAV flight missions.

Furthermore, chapter 3 outlined the challenging situation to find an optimum balance between the demands of various actors, allowing for innovation on the one hand and ensuring recognition and support for safety, fundamental human rights, and civil liberties. The future development of UAV applications will ultimately involve multiple interest groups and various motivations (Rao, Gopi and Maione, 2016). It can be predicted that over the next decade, technology, societal acceptance and regulation may converge. Reflecting on chapter 3 , remarkable progress has already been made during the past years. More and more countries are establishing risk-based regulations as a fundamental basis to unlock the full potential of UAVs for national economies. The bottom line is that all users should comply with the rules and regulations, even though compliance assessment and compliance finding might be in the early stages of development (Washington, Clothier and Silva, 2019). Otherwise, if widely publicised incidents happen, the risk-based system might get jeopardised, and the current balance for regulating UAV missions might be revisited and even lead to a 'no, unless ..' system in many more cases. 


\subsection{Limitations of this work}

Several aspects limited the scope of the research and required data acquisition in this thesis. At the beginning of this work, controlled large scale UAV test flights and extensive community engagement in Rwanda, Kenya and Ethiopia were foreseen. However, external forces and a strong dependency on policy and (absence of) regulations, UAV pilots, and technology significantly challenged these plans. Firstly, Ethiopia faced a severe political crisis during the first phase of our work, and it was impossible to import UAV technology or even do fieldwork in the pilot region. Although the Rwandan UAV legislation got enacted in March 2016 and pretended straightforward procedures for UAV registration, pilot certification, and flight approval, the time required to obtain all necessary documentation to conduct UAV flights with our Rwandese project partners exceeded the time of the its4land project, and an existing UAV company had to be contracted. In Kenya, flight approval was granted only one time via exemption regulations for six weeks. Overall, these circumstances limited the spatial extent and the level of control during the envisaged test flights. Additionally, the study location was extended to the Republic of Tanzania to derive additional data.

On the flip side, the limitations we experienced marked a solid motivation to look at the diffusion of technology and investigate the potentials and the challenges of scaling the use of UAV technology in land administration, as outlined in chapter 5 .

\subsection{Prospects for future research}

This thesis showed the opportunities and limitations of UAV technology for responsible land administration by undertaking research investigations on land data needs, UAV regulations and data quality aspects. However, several questions remain unanswered at present.

Further research will be needed to explore the overall costs of UAV compared to other geospatial technologies such as satellite data and classical aerial photographs in providing base data. A robust understanding of the costs and time resources allows inferring on costbenefit calculations and assessing the profitability of UAV technology in an operational environment

Following this aspect, it would be interesting to analyse the impact of UAV regulations on the development of businesses. Furthermore, investigations of likely consequences for market developments in 
different regulatory contexts could be another angle for additional research.

The proposed model of the importance of FELA pathways, as presented in chapter 5 , provides a baseline for future work focussing on national contexts. A comparison between the generalized model and contextspecific peculiarities at the national level and potentially different ways to reach the plateau of productivity would be fascinating. 


\section{Bibliography}

Adams, S. M. and Friedland, C. J. (2011) 'A Survey of Unmanned Aerial Vehicle (UAV) Usage for Imagery Collection in Disaster Research and Management', in Proceedings of 9th International Workshop on Remote Sensing for Disaster Response. Stanfort University, California, USA.

Aditya, T. et al. (2020) 'Participatory land administration in Indonesia: Quality and usability assessment', Land, 9(3), pp. 1-27.

Agüera-Vega, F., Carvajal-Ramírez, F. and Martínez-Carricondo, P. (2017) 'Assessment of photogrammetric mapping accuracy based on variation ground control points number using unmanned aerial vehicle', Measurement, 98, pp. 221-227.

Alcantarilla, P. F., Nuevo, J. and Bartoli, A. (2013) 'Fast explicit diffusion for accelerated features in nonlinear scale spaces', in BMVC 2013 - Electronic Proceedings of the British Machine Vision Conference 2013.

Asiama, K., Bennett, R. and Zevenbergen, J. (2017) 'Participatory Land Administration on Customary Lands: A Practical VGI Experiment in Nanton, Ghana', ISPRS International Journal of GeoInformation.

Barnes, G. et al. (2014) 'Drones for Peace: Part 1 of 2 Design and Testing of a UAV-based Cadastral Surveying and Mapping Methodology in Albania. Paper prepared for presentation at the 2014 WORLD BANK CONFERENCE ON LAND AND POVERTY

Barnes, G. and Volkmann, W. (2015) 'High-Resolution Mapping with Unmanned Aerial Systems', Surveying and Land Information Science, 74(1), pp. 5-13.

Bay, H. et al. (2008) 'Speeded-Up Robust Features (SURF)', Computer Vision and Image Understanding, 110(3), pp. 346-359.

Benassi, F. et al. (2017) 'Testing accuracy and repeatability of UAV blocks oriented with gnss-supported aerial triangulation', Remote Sensing, 9(2).

Bennett Moses, L. (2007) 'Recurring Dilemmas: The Laws's Race to Keep Up with Technological Change', Journal of Law, Technology \& Policy, 2, pp. 239-285.

Bennett Moses, L. (2013) 'How to Think about Law, Regulation and Technology: Problems with "Technology" as a Regulatory Target', Law Innovation and Technology, 1(June), pp. 1-20. 
Bennett, R. et al. (2013) 'Continuing To Bridge the Cadastral Divide', Annual World Bank Conference on Land and Poverty, pp. 1-22.

Bennett, R. et al. (2021) 'Recent land administration experiences from the Arab region: Findings from Egypt, Lebanon and the Palestinian Territories', in Second Arab Land Conference.

Bennett, R. M., Pickering, M. and Sargent, J. (2019) 'Transformations, transitions, or tall tales? A global review of the uptake and impact of NoSQL, blockchain, and big data analytics on the land administration sector', Land Use Policy. Elsevier, pp. 435-448.

Besley, T. (1995) 'Property Rights and Investment Incentives: Theory and Evidence from Ghana. The University of Chicago Press, 103(5), pp. 903-937.

Bleijenbergh, I., Korzilius, H. and Verschuren, P. (2011) 'Methodological criteria for the internal validity and utility of practice oriented research', Quality \& Quantity, 45(1), pp. 145156.

van Blyenburgh, P. (2016) 'Harmonising UAS Regulations and Standards', GIM International - UAS Special Issue. Lemmer, The Netherlands: Geomares, 30.

Boucher, P. (2015) 'Domesticating the Drone: The Demilitarisation of Unmanned Aircraft for Civil Markets', Science and Engineering Ethics, 21(6), pp. 1393-1412.

Cantrill, J. A., Sibbald, B. and Buetow, S. (1996) 'The Delphi and nominal group techniques in health services research The Delphi technique', The International Journal of Pharmacy Practice, June, pp. 67-74.

Casiano Flores, C. et al. (2020) 'Governance assessment of the UAVs implementation in Rwanda under the fit-for-purpose land administration approach', Land Use Policy.

Cavoukian, A. (2012) Privacy and Drones: Unmanned Aerial Vehicles, Privacy by Design, Canada. Ontario, Canada.

Chan, T. O. and Williamson, I. P. (1999) 'The different identities of GIS and GIS diffusion', International Journal of Geographical Information Science, 13(3), pp. 267-281.

Cheney, C. (2019) Drones for development? A DEVEX Report, DEVEX.

Clarke, R. (2014) 'What drones inherit from their ancestors', Computer Law and Security Review. Elsevier Ltd, 30(3), pp. 247-262. 
Clarke, R. and Bennett Moses, L. (2014) 'The regulation of civilian drones' impacts on behavioural privacy', Computer Law and Security Review. Elsevier Ltd, 30(3), pp. 286-305.

Clothier, R. A. et al. (2011) 'Definition of an airworthiness certification framework for civil unmanned aircraft systems', Safety Science.

Clothier, R. A. et al. (2015) 'Risk Perception and the Public Acceptance of Drones', Risk Analysis, 35(6), pp. 1167-1183.

Clothier, R., Fulton, N. and Walker, R. (2008) 'Pilotless aircraft: the horseless carriage of the twenty-first century?', Journal of Risk Research, 11(8), pp. 999-1023.

Colomina, I. and Molina, P. (2014) 'Unmanned aerial systems for photogrammetry and remote sensing: A review', ISPRS Journal of Photogrammetry and Remote Sensing. International Society for Photogrammetry and Remote Sensing, Inc. (ISPRS), 92, pp. 79-97.

Cooper, H. and Hedges, L. V (2009) 'Research synthesis as a scientific process', The handbook of research synthesis and meta-analysis, pp. 3-17.

Crommelinck, S. et al. (2018) 'Interactive cadastral boundary delineation from UAV data', in ISPRS Annals of Photogrammetry, Remote Sensing and Spatial Information Sciences, pp. 4-7.

Crommelinck, S. et al. (2019) 'Application of deep learning for delineation of visible cadastral boundaries from remote sensing imagery', Remote Sensing, 11(21).

Crommelinck, S. (2019) Delineation tool. GitHub. Available at: https://github.com/SCrommelinck/delineation-tool (Accessed: 10 July 2020).

Davison, R. et al. (2000) 'Technology Leapfrogging in Developing Countries - An Inevitable Luxury?', The Electronic Journal of Information Systems in Developing Countries, pp. 1-10.

Dedehayir, O. and Steinert, M. (2016) 'The hype cycle model : A review and future directions', Technological Forecasting \& Social Change. Elsevier Inc., 108, pp. 28-41.

Delbecq, A. L. and Van de Ven, A. H. (1971) 'A group process model for problem identification and program planning', The Journal of Applied Behavioral Science. Sage PublicationsSage CA: Thousand Oaks, CA, 7(4), pp. 466-492.

Delbecq, A. L., Van de Ven, A. H. and Gustafson, D. H. (1975) Group techniques for program planning: a guide to nominal group and Delphi processes. Glenview, Ill. : Scott, Foresman. 
Devriendt, L. and Bonne, J. (2014) 'UAS mapping as an alternative for land surveying techniques?', International Archives of the Photogrammetry, Remote Sensing and Spatial Information Sciences - ISPRS Archives, 40(3W1), pp. 39-45.

Dijkstra, P. et al. (2020) 'Towards Land Sector Equity and Resilience in Nepal - Pole, Uptake and Impact of UAVs', in World Bank Land and Poverty Conference. Washington D.C.

DRONEII (2019) The Drone Industry Barometer 2019.

EASA (2015) Riga Declaration on Remotely Piloted Aircraft (drones). Riga, Latvia.

EASA (2016) 'Prototype' Commission Regulation on Unmanned Aircraft Operations - Explanatory Note.

Eisenbeiß, H. (2009) 'UAV photogrammetry', Institute of Photogrammetry and Remote Sensing. PhD Thesis.

Ekaso, D., Nex, F. and Kerle, N. (2020) 'Accuracy assessment of realtime kinematics (RTK) measurements on unmanned aerial vehicles (UAV) for direct geo-referencing', Geo-Spatial Information Science. Taylor \& Francis, 23(2), pp. 165-181.

Eling, C., Klingbeil, L. and Kuhlmann, H. (2014) 'Development of an RTK-GPS system for precise real-time positioning of lightweight UAVs', Ingenieurvermessung, (i), pp. 1-12.

Eltner, A. et al. (2015) 'Image-based surface reconstruction in geomorphometry - merits, limits and developments of a promising tool for geoscientists', Earth Surface Dynamics Discussions, pp. 1445-1508.

Enemark, S. (2004) 'Building Land Information Policies', UN, FIG, PC IDEA Inter-regional Special Forum on The Building of Land Information Policies in the Americas, (October), pp. 1-20.

Enemark, S. et al. (2014) Fit-For-Purpose Land Administration, International Federation of Surveyors (FIG).

Enemark, S. et al. (2016) 'Guiding principles for building fit-forpurpose land administration systems in developing countries', in Scaling up Responsible Land Governance.

European Commission (2016) Responsible research and innovation (RRI).

European Commission (2019) Commission Implementing Regulation (EU) 2019/947 on the rules and procedures for the operation of unmanned aircraft. 
European Parliament (2018) Regulation (EU) 2018/1139 on common rules in the field of civil aviation and establishing a European Union Aviation Safety Agency.

European RPAS Steering Group (2013) 'Roadmap for the integration of civil Remotely - Piloted Aircraft Systems into the European Aviation System', (June), pp. 1-54.

Everaerts, J. (2009) New Platforms - Unconventional Platforms (Unmanned Aircraft Systems) for Remote Sensing. Amsterdam, The Netherlands.

Federal Aviation Administration (2016) Operation and Certification of Small Unmanned Aircraft Systems.

Fetai, B. et al. (2019) 'Extraction of visible boundaries for cadastral mapping based on UAV imagery', Remote Sensing, 11(13).

Feyertag, J. et al. (2020) A global assessment of perceived tenure security from 140 countries.

Figliozzi, M. A., Tucker, C. and Polikakhina, P. (2018) 'Drone deliveries logistics, efficiency, safety and last mile trade-offs', in ILS 2018 - Information Systems, Logistics and Supply Chain, Proceedings.

Finn, R. L. and Wright, D. (2012) 'Unmanned aircraft systems: Surveillance, ethics and privacy in civil applications', Computer Law and Security Review. Elsevier Ltd, 28(2), pp. 184-194.

Finn, R. L. and Wright, D. (2014) Study on privacy, data protection and ethical risks in civil Remotely Piloted Aircraft Systems operations. Brussels, Belgium: European Comission.

Finn, R. L. and Wright, D. (2016) 'Privacy, data protection and ethics for civil drone practice: A survey of industry, regulators and civil society organisations', Computer Law and Security Review. Elsevier Ltd.

Floreano, D. and Wood, R. J. (2015) 'Science, technology and the future of small autonomous drones', Nature, 521(7553), pp. 460-466.

Forlani, G. et al. (2018) 'Quality assessment of DSMs produced from UAV flights georeferenced with on-board RTK positioning', Remote Sensing, 10(2), pp. 1-22.

Förstner, W. and Gülch, E. (1987) 'A Fast Operator for Detection and Precise Location of Distinct Points, Corners and Centres of Circular Features', ISPRS Intercommission Workshop, pp. 281305. 
Foster, R. N. (1986) 'Working the S-curve: assessing technological threats', Research Management. Taylor I\& Francis, 29(4), pp. $17-20$.

Freeman, P. K. and Freeland, R. S. (2015) 'Agricultural UAVs in the U.S.: Potential, policy, and hype', Remote Sensing Applications: Society and Environment. Elsevier, 2, pp. 35-43.

Gartner Inc. (2019) Hype Cycle for Drones and Mobile Robots, 2019.

Gartner Inc. (2021) Gartner. Available at: https://www.gartner.com/en (Accessed: 27 January 2021).

Gerke, M. and Przybilla, H. J. (2016) 'Accuracy Analysis of Photogrammetric UAV Image Blocks: Influence of Onboard RTKGNSS and Cross Flight Patterns', Photogrammetrie Fernerkundung - Geoinformation, pp. 17-30.

Gillingham, P. and Buckle, F. (2014) Rwanda Land Tenure Regularisation Case Study.

Giones, F. and Brem, A. (2017) 'From toys to tools: The co-evolution of technological and entrepreneurial developments in the drone industry', Business Horizons. 'Kelley School of Business, Indiana University', 60(6), pp. 875-884.

GLTN (2012) Handling land: Innovative tools for land governance and secure tenure. Nairobi.

Gonzales-Aguilera, D. et al. (2020) 'Photomatch: an open-source multi-view and multi-modal feature matching tool for photogrammetric applications', ISPRS Annals of Photogrammetry, Remote Sensing and Spatial Information Sciences.

Grant, D. et al. (2020) 'The Cadastral triangular model', Land Use Policy, 97.

Grenzdörffer, G., Engel, A. and Teichert, B. (2008) 'The photogrammetric potential of low-cost UAVs in forestry and agriculture', in International Archives of Photogrammetry Remote Sensing and Spatial Information Sciences Vol. XXXVII. Part B1. Beijing, China: ISPRS, pp. 1207-1213.

Gruen, A. (2012) 'Development and Status of Image Matching in Photogrammetry', Photogrammetric Record, 27(137), pp. 36-57.

Haala, N. et al. (2012) 'Performance Test on Uav-Based Photogrammetric Data Collection', ISPRS - International Archives of the Photogrammetry, Remote Sensing and Spatial Information Sciences, XXXVIII-1/(September), pp. 7-12. 
Hardiono, M. et al. (2016) 'Empowering Communities to Mark Boundaries and Map Resources with Geospatial Technology: Early Results of using UAVs in Participatory Village Boundary Setting / Resource Mapping (VBS/RM) activity in Indonesia', in Land and Poverty Conference 2016: Scaling up Responsible Land Governance.

Hargadon, A. B. and Douglas, Y. (2001) 'When Innovations Meet Institutions: Edison and the Design of the Electric Light', Administrative Science Quarterl, 46.

Haula, K. and Agbozo, E. (2020) 'A systematic review on unmanned aerial vehicles in Sub-Saharan Africa: A socio-technical perspective', Technology in Society. Elsevier Ltd, 63, p. 101357.

Hendriks, B. et al. (2019) 'Pro-poor land administration: Towards practical, coordinated, and scalable recording systems for all', Land Use Policy. Elsevier Ltd, 81, pp. 21-38.

Ho, S. et al. (2013) 'Land Use Policy Legal barriers to 3D cadastre implementation: What is the issue?', Land Use Policy. Elsevier Ltd, 35, pp. 379-387.

Ho, S. et al. (2019) 'Scaling emerging geospatial technologies for land administration: understanding institutional innovation dynamics through a Technological Innovation System perspective', World Bank Land and Poverty Conference, (July).

Honkavaara, E. et al. (2012) 'Hyperspectral Reflectance Signatures and Point Clouds for Precision Agriculture By Light Weight Uav Imaging System', in Annals of Photogrammetry, Remote Sensing and Spatial Information Sciences. Melboure, Australia: ISPRS, pp. 353-358.

Hsieh, H. F. and Shannon, S. E. (2005) 'Three approaches to qualitative content analysis', Qualitative Health Research, 15(9), pp. 12771288.

Hugenholtz, C. et al. (2016) 'Spatial accuracy of UAV-derived orthoimagery and topography: Comparing photogrammetric models processed with direct geo-referencing and ground control points', Geomatica, 70(1).

ICAO (1944) Convention on International Civil Aviation. USA: ICAO.

ICAO (2011) Unmanned Aircraft Systems (UAS), Cir 328 AN/190. Montreal, Canada: ICAO.

ICAO (2015) Manual on Remotely Piloted Aircraft Systems (RPAS). Montréal, Quebec, Canada: ICAO. 
ISO (2013) 'ISO 19157: Geographic information-data quality.' International Organization for Standardization.

Ison, R., Röling, N. and Watson, D. (2007) 'Challenges to science and society in the sustainable management and use of water: investigating the role of social learning', Environmental Science and Policy, 10(6), pp. 499-511.

James, M. R. et al. (2014) 'Mitigating systematic error in topographic models derived from UAV and ground-based image networks', 1420(June), pp. 1413-1420.

James, M. R. et al. (2017) 'Optimising UAV topographic surveys processed with structure-from-motion: Ground control quality, quantity and bundle adjustment', Geomorphology. Elsevier B.V., 280, pp. 51-66.

Jansen, P. (2015) The ethics of domestic drones - an evaluation of the use of Surveillance-Capable Unmanned Aerial Systems in Civil Contexts. University of Twente.

JARUS (2013) Certification Specification for Light Unmanned Rotorcraft Systems.

JARUS (2015) FCL Recommendation.

JARUS (2016) RPAS "Required C2 Performance "( RLP) concept.

Jepsen, M. R. et al. (2015) 'Transitions in European land-management regimes between 1800 and 2010', Land Use Policy, 49, pp. 5364.

Joint Aviation Authorities and Eurocontrol (2004) A Concept for European Regulations for Civil Umanned Aerial Vehicles (UAVs).

Kelm, K. et al. (2014) 'Drones for Peace: Part II Fast and Inexpensive Spatial Data Capture for Multi - Purpose Use', pp. 1-22.

Kelm, K. (2014) 'UAVs Revolutionise Land Administration', GIM International, p. 2014.

Koeva, M. et al. (2016) 'Using UAVs for map creation and updating. A case study in Rwanda', Survey Review. Taylor \& Francis, 50(361), pp. 1-14.

Koeva, M. et al. (2017) 'Towards innovative geospatial tools for fit-forpurpose land rights mapping', in International Archives of the Photogrammetry, Remote Sensing and Spatial Information Sciences - ISPRS Archives, pp. 37-43.

Koeva, M. et al. (2020) 'Innovative remote sensing methodologies for Kenyan land tenure mapping', Remote Sensing, 12(2). 
Koeva, M. et al. (2021) 'Fit-for-Purpose Land Administration Assessment', Land, 10(557), pp. 1-23.

Kohli, D., Unger, E. and Lemmen, C. (2018) 'Validation of a cadastral map created using satellite imagery and automated feature extraction techniques: A case of Nepal', in FIG Congress 2018: Embracing our smart world where the continents connect: enhancing the geospatial maturity of societies. Istanbul.

Kshetri, N. (2017) 'Will blockchain emerge as a tool to break the poverty chain in the Global South?', Third World Quarterly. Routledge, 38(8), pp. 1710-1732.

Kumar, R. (2014) Research Methodology, New Age International. SAGE.

Langford, B. E., Schoenfeld, G. and Izzo, G. (2002) 'Nominal grouping sessions vs focus groups', Qualitative Market Research: An International Journal. Emerald, 5(1), pp. 58-70.

Leavitt, H. J. (1965) 'Applied Organizational Change in Industry: Structural, Technological and Humanistic Approaches', in March, J. G. (ed.) Handbook of Organizatinos. Illinois: Rand McNally and Company.

Lee, J. K., Kang, sang G. and Lee, D. (2016) 'A study on the verification of the UAV utilization for Cadastral Renovation Project', in Land and Poverty Conference 2016: Scaling up Responsible Land Governance.

Lemmen, C. et al. (2015) 'A New Era in Land Administration Emerges', Geomares Publishing, 29(1).

Lengoiboni, M., Richter, C. and Zevenbergen, J. (2019) 'Cross-cutting challenges to innovation in land tenure documentation', Land Use Policy. Elsevier, 85(March), pp. 21-32.

Liddell, W. W. (2004) 'A Socio-Technical Systems Analysis of Capital Markets'.

Lijphart, A. (1971) 'Comparative Politics and the Comparative Method', The American Political Science Review, 65(3), pp. 682-693.

Linden, A. and Fenn, J. (2003) Understanding Gartner' s Hype Cycles Understanding Gartner's Hype Cycles, Strategic Analysis Report.

Lloyd, S. (2011) 'Applying the nominal group technique to specify the domain of a construct', Qualitative Market Research: An International Journal. Emerald Group Publishing Limited, 14(1), pp. $105-121$. 
Lowe, D. G. (1999) 'Object recognition from local scale-invariant features', Proceedings of the IEEE International Conference on Computer Vision, 2, pp. 1150-1157.

Lowe, G. (2004) 'SIFT - The Scale Invariant Feature Transform', 2, pp. 91-110.

Lu, C., Xu, L. and Jia, J. (2012) 'Contrast preserving decolorization', 2012 IEEE International Conference on Computational Photography, ICCP 2012.

Manfreda, S. et al. (2019) 'Assessing the Accuracy of Digital Surface Models Derived from Optical Imagery Acquired with Unmanned Aerial Systems', Drones, 3(1), pp. 1-14.

Manyoky, M. et al. (2012) 'Unmanned Aerial Vehicle in Cadastral Applications', ISPRS - International Archives of the Photogrammetry, Remote Sensing and Spatial Information Sciences, XXXVIII-1/(September), pp. 57-62.

Marchant, G. E., Allenby, B. R. and Herkert, J. R. (2011) The growing gap between emerging technologies and legal-ethical oversight: The pacing problem. 7th edn. New York, USA: Springer.

Marzocchi, O. (2015) Privacy and Data Protection Implications of the Civil Use of Drones, European Parliament. Brussels, Belgium.

Matese, A. et al. (2015) 'Intercomparison of UAV, aircraft and satellite remote sensing platforms for precision viticulture', Remote Sensing, 7(3), pp. 2971-2990.

Maurice, M. J. et al. (2015) 'A photogrammetric approach for map updating using UAV in Rwanda .', in GeoTechRwanda. Kigali, pp. $1-8$.

Maza, I. et al. (2011) 'Experimental results in multi-UAV coordination for disaster management and civil security applications', Journal of Intelligent and Robotic Systems: Theory and Applications, 61(1-4), pp. 563-585.

Meha, M. et al. (2016) 'UAV Technology and Open Source Software for Systematic registration in the Republic of Kosovo', in Land and Poverty Conference 2016: Scaling up Responsible Land Governance.

Mesas-Carrascosa, F. J. et al. (2014) 'Validation of measurements of land plot area using UAV imagery', International Journal of Applied Earth Observation and Geoinformation, 33(1), pp. 270279.

Mesas-Carrascosa, F. J. et al. (2015) 'Assessing optimal flight parameters for generating accurate multispectral orthomosaicks 
by uav to support site-specific crop management', Remote Sensing, 7(10), pp. 12793-12814.

Meticulous Research (2019) Unmanned Aerial Vehicle (UAV) Market Global Opportunity Analysis and Industry Forecasts (2020-2027).

Montuori, A. (2011) 'Systems Approach', In: Runco, M. A. and S. Pritzker, (eds). Encyclopedia of Creativity (Second Edition), San Diego: Academic Press, pp. 414-421.

Morales, A. C., Paez, D. and Arango, C. (2015) 'Multi-criteria analysis of UAVs regulations in 6 countries using the analytical hierarchical process and expert knowledge', in International Conference on Unmanned Aerial Vehicles in Geomatics. Toronto, Canada: ISPRS, pp. 175-181.

Mumbone, M. et al. (2015) 'Innovations in boundary mapping: Namibia, customary lands and UAVs', in Land and Poverty Conference 2015: Linking Land Tenure and Use for Shared Prosperity.

National Institute of Statistics Rwanda (2014) Fourth Population and Housing Census, 2012 Census Atlas. Rwanda.

Nepal Flying Labs (2020) Workshop to design a flight authorization platform. Available at: https://blog.werobotics.org/2020/12/07/launching-a-digitalsolution-to-expedite-drone-flight-permissions-for-disasterresponse/.

Nex, F. and Remondino, F. (2014) 'UAV for 3D mapping applications: A review', Applied Geomatics, 6(1), pp. 1-15.

Ngoga, T. H. (2018) Rwanda 's Land Tenure Reform. CABI.

Oniga, V. E. et al. (2020) 'Determining the suitable number of ground control points for UAS images georeferencing by varying number and spatial distribution', Remote Sensing, 12(5).

Ottens, M. and Stubkjær, E. (2007) 'A socio-technical analysis of cadastral systems', in Zevenbergen, J., Frank, A., and Stubkjær, E. (eds) Real property transactions. IOS Press.

Panday, U. S. et al. (2021) 'Securing Land Rights for All through Fitfor-Purpose Land Administration Approach: The Case of Nepal', pp. 1-24.

Pfeifer, N., Glira, P. and Briese, C. (2012) 'Direct georeferencing with on board navigation components of light weight UAV platforms', International Archives of the Photogrammetry, Remote Sensing and Spatial Information Sciences - ISPRS Archives, 39(September), pp. 487-492. 
Pont-Tuset, J. et al. (2017) 'Multiscale Combinatorial Grouping for Image Segmentation and Object Proposal Generation', IEEE Transactions on Pattern Analysis and Machine Intelligence, 39(1), pp. 128-140.

Potter, W. J. and Levine-Donnerstein, D. (1999) 'Rethinking validity and reliability in content analysis', Journal of Applied Communication Research, 27(3), pp. 258-284.

PWC (2018) Drones impact on UK economy, PWC.

Radovic, M. (2019) 'Leapfrogging into the Future: Drones in SubSaharan Africa', DRONEII.

Rahmatizadeh, S. et al. (2018) 'A framework for selecting a fit-forpurpose data collection method in land administration', Land Use Policy. Elsevier, 70(November 2017), pp. 162-171.

Ramadhani, S. A., Bennett, R. M. and Nex, F. C. (2018) 'Exploring UAV in Indonesian cadastral boundary data acquisition', Earth Science Informatics. Earth Science Informatics, 11(1), pp. 129-146.

Rango, A. and Laliberte, A. S. (2010) 'Impact of flight regulations on effective use of unmanned aircraft systems for natural resources applications', Journal of Applied Remote Sensing, 4(1), p. 043539.

Rao, B., Gopi, A. G. and Maione, R. (2016) 'The societal impact of commercial drones', Technology in Society. Elsevier Ltd, 45, pp. 83-90.

Rehak, M. and Skaloud, J. (2016) 'Applicability of New Approaches of Sensor Orientation To Micro Aerial Vehicles', ISPRS Annals of Photogrammetry, Remote Sensing and Spatial Information Sciences, III-3(July), pp. 441-447.

Remondino, F. et al. (2011) 'Uav Photogrammetry for Mapping and 3D Modeling - Current Status and Future Perspectives -', International Archives of the Photogrammetry, XXXVIII(September), pp. 14-16.

Remondino, F. et al. (2014) 'State of the art in high density image matching', Photogrammetric Record, 29(146), pp. 144-166.

Rijsdijk, M. et al. (2013) 'Unmanned Aerial Systems in the Process of Juridical Verification of Cadastral Border', International Archives of Photogrammetry and Remote Sensing, XL(September), pp. 46.

Rogers, E. M. (1995) Diffusion of innovations. New York. 
Rosen, J. (2017) 'Zipline's Ambitious Medical Drone Delivery in Africa', MIT Technology Review. Available at: https://www.technologyreview.com/s/608034/blood-from-thesky-ziplines-ambitious-medical-drone-delivery-in-africa/.

Rubinov, E. et al. (2015) 'From Barefoot to "Air-foot" surveyors', GIM International. Geomatics Information and Trading Centre B.V., 29(6), pp. 21-23.

Rwanda Civil Aviation Authority (2016) Ministerial Regulations No 01/MOS/Trans/016 of 26/04/2016 Relating to Unmanned Civil Aircraft System.

Rwanda Civil Aviation Authority (2018) 'Ministerial Regulations No 01/MOS/Trans/2018 of 23/01/2018 Relating to Unmanned Civil Aircraft System', pp. 30-70.

Santos, F., Fletschner, D. and Daconto, G. (2014) 'Enhancing Inclusiveness of Rwanda's Land Tenure Regularization Program: Insights from Early Stages of its Implementation', World Development. Elsevier Ltd, 62, pp. 30-41.

Sanz-Ablanedo, E. et al. (2018) 'Accuracy of Unmanned Aerial Vehicle (UAV) and SfM photogrammetry survey as a function of the number and location of ground control points used', Remote Sensing, 10(10).

Seifert, E. et al. (2019) 'Influence of drone altitude, image overlap, and optical sensor resolution on multi-view reconstruction of forest images', Remote Sensing, 11(10).

Singh, K. K. and Frazier, A. E. (2018) 'A meta-analysis and review of unmanned aircraft system (UAS) imagery for terrestrial applications', International Journal of Remote Sensing. Taylor \& Francis, 39(15-16), pp. 5078-5098.

Sink, D. S. (1983) 'Using the nominal group technique effectively', National Productivity Review. Wiley-Blackwell, 2(2), pp. 173184.

Snavely, N., Steven, M., Seitz, R. S. (2006) 'Photo Tourism: Exploring image collections in 3D', Proceedings of SIGGRAPH 2006, 1(212), pp. 835-846.

South African Civil Aviation Authority (2016) Technical guidance material for RPAS part 101.

Spectairgroup (2016) 'UAS Experiences in Africa: Heat, Dust and Distrustful Locals', GIM International, pp. 3-4.

Stöcker, C., Nex, F., et al. (2017) 'Quality assessment of combined IMU/GNSS data for direct georeferencing in the context of UAV- 
Bibliography

based mapping', in International Archives of the Photogrammetry, Remote Sensing and Spatial Information Sciences - ISPRS Archives, pp. 355-361.

Stöcker, C., Bennett, R., et al. (2017) 'Review of the current state of UAV regulations', Remote Sensing, 9(5).

Stöcker, C. et al. (2018) 'Towards UAV-based Land Tenure Data Acquisition in Rwanda: Needs Assessment and Technology Response Towards UAV-based Land Tenure Data Acquisition in Rwanda: Needs Assessment and Technology Response', in FIG Congress 2018: Embracing our smart world where the continents connect: enhancing the geospatial maturity of societies. Istanbul.

Stöcker, C., Koeva, M., et al. (2019) 'Evaluation of UAV-based technology to capture land rights in Kenya: Displaying stakeholder perspectives through interactive gaming', in World Bank Conference on Land and Poverty. Washington D.C.

Stöcker, C., Ho, S., et al. (2019) 'Unmanned Aerial System Imagery, Land Data and User Needs: A Socio-Technical Assessment in Rwanda', Remote Sensing, 11(9), p. 1035.

Stöcker, C. et al. (2020) 'High-quality UAV-based orthophotos for cadastral mapping: Guidance for optimal flight configurations', Remote Sensing.

Stöcker, C., Koeva, M. and Zevenbergen, J. (2020) 'UAV Technology: Opportunities to Support the Updating Process of the Rwandan Cadastre', in FIG Working Week 2020, pp. 1-14.

Tampubolon, W. and Reinhardt, W. (2014) 'UAV Data Processing for Large Scale Topographical Mapping', ISPRS - International Archives of the Photogrammetry, Remote Sensing and Spatial Information Sciences. International Society for Photogrammetry and Remote Sensing, XL-5(5), pp. 565-572.

Tan, E., Flores, C. and Crompvoets, J. (2021) 'Land Use Policy A capacity assessment framework for the fit-for-purpose land administration systems: The use of unmanned aerial vehicle ( UAV ) in Rwanda and Kenya', 102(December 2020).

Tashakkori, A. and Teddlie, C. (1998) Mixed methodology: combining qualitative and quantitative approaches. 46th edn. SAGE.

Tatham, P. (2009) 'An investigation into the suitability of the use of unmanned aerial vehicle systems', International Journal of Risk Assessment and Management, 13(1), pp. 60-78.

The Japan Times (2016) 'Man who landed drone on roof of Japanese prime minister's office gets suspended sentence'. Available at: 
http://www.japantimes.co.jp/news/2016/02/16/national/crimelegal/man-landed-drone-roof-japanese-prime-ministers-officegets-suspended-sentence/\#.WFEvqIrQf_w (Accessed: 17 December 2016).

Tonkin, T. N. and Midgley, N. G. (2016) 'Ground-control networks for image based surface reconstruction: An investigation of optimum survey designs using UAV derived imagery and structure-frommotion photogrammetry', Remote Sensing, 8(9), pp. 16-19.

Turner, D., Lucieer, A. and Wallace, L. (2014) 'Direct georeferencing of ultrahigh-resolution UAV imagery', IEEE Transactions on Geoscience and Remote Sensing, 52(5), pp. 2738-2745.

UAViators (2015) Humanitarian UAV Code of Conduct \& Guidelines. UN-DESA (2019) World Population Prospects 2019. New York, USA.

UN-FAO (2012) Voluntary Guidelines on the Responsible Governance of Tenure of Land, Fisheries and Forests in the Context of National Food Security.

UN-GGIM (2015) 'The Application of Geospatial Information - Land Administration and Management', (July), pp. 1-28.

UN-HABITAT (2008) Secure Land Rights for All, Global Land Tool Network. Available at: www.unhabitat.org.

UN-Habitat and GLTN (2015) Property Theory, Metaphors and the Continuum of Land Rights.

UN (2015) 'Transforming our world: the 2030 Agenda for Sustainable Development Goal', pp. 1-35.

UNDP and Government of Rwanda (2015) Rwanda National Human Development Report 2014 (March 2015).

UNECE (1996) Land Administration Guidelines. Available at: http://www.unece.org/fileadmin/DAM/hlm/documents/Publicatio ns/land.administration.guidelines.e.pdf.

UNGGIM (2020) 'Framework for Effective Land Administration', (July). Union African and NEPAD (2018) Drones on the horizon - transforming African's horizon.

Villanueva, J. K. S. and Blanco, A. C. (2019) 'Optimization of ground control point (GCP) configuration for unmanned aerial vehicle (UAV) survey using structure from motion (SFM)', International Archives of the Photogrammetry, Remote Sensing and Spatial Information Sciences - ISPRS Archives, 42(4/W12), pp. 167-174.

Villasenor, J. (2013) 'Observations from above: unmanned aircraft systems and privacy', Harv. JL \& Pub. Pol'y, 36. 
De Vries, W. and Chigbu, U. E. (2017) 'Responsible Land Management - Concept and application in a ter- ritorial rural context', FuB Flächenmanagement und Bodenordnung, (2), pp. 65-73.

de Vries, W. T., Bennett, R. M. and Zevenbergen, J. A. (2015) 'Toward Responsible Land Administration', in Advances in Responsible Land Administration, pp. 3-14.

Washington, A., Clothier, R. and Silva, J. (2019) 'Challenges to the Risk-based Regulation of Unmanned Aircraft Systems', Australian International Aerospace Congress (AIAC18), (August).

Watts, A. C., Ambrosia, V. G. and Hinkley, E. A. (2012) 'Unmanned aircraft systems in remote sensing and scientific research: Classification and considerations of use', Remote Sensing, 4(6), pp. 1671-1692.

WBI Evaluation Group (2007) 'Nominal Group Technique', World Bank Needs Assessment Knowledge Base. The World Bank.

van Wegen, W. and Stumpf, J. (2016) 'Bringing a new level of intelligence to UAVs - Interview with Jan Stumpf', GIM International - UAS Special Issue. Lemmer, The Netherlands: Geomares.

Widodo, S. et al. (2016) 'Empowering Communities to Mark Boundaries and Map Resources with Geospatial Technology: Early Results of using UAVs in Participatory Village Boundary Setting / Resource Mapping (VBS/RM) activity in Indonesia', in Land and Poverty Conference 2016: Scaling up Responsible Land Governance. Washington D.C.

Williamson, I. et al. (2010) 'Land Administration for Sustainable Development', Redlands, CA: ESRI Press Academic.

Witkin, B. R. and Altschuld, J. W. (1995) Planning and Conducting Needs Assessments: A Practical Guide. Thousand Oaks, CA: SAGE Publications Inc.

Xia, X., Persello, C. and Koeva, M. (2019) 'Deep fully convolutional networks for cadastral boundary detection from UAV images', Remote Sensing, 11(14).

Yin, R. K. (2003) 'Case study research: design and methods', Applied Social research Methods Series. Third Edit.

Yu, X. and Zhang, Y. (2015) 'Sense and avoid technologies with applications to unmanned aircraft systems: Review and prospects', Progress in Aerospace Sciences. Elsevier, 74, pp. $152-166$. 
Zeng, Z. and Cleon, C. B. (2018) 'Factors affecting the adoption of a land information system: An empirical analysis in Liberia', Land Use Policy, 73, pp. 353-362.

Zevenbergen, J. (2004) 'A Systems Approach to Land Registration and Cadastre', Nordic Journal of Surveying and Real Estate Research, 1.

Zevenbergen, J. et al. (2013) 'Pro-poor land administration: Principles for recording the land rights of the underrepresented', Land Use Policy. Elsevier Ltd, 31, pp. 595-604.

Zevenbergen, J., De Vries, W. T. and Bennett, R. M. (2015) Advances in Responsible Land Administration. CRC Press.

Zhang, C. and Kovacs, J. M. (2012) 'The application of small unmanned aerial systems for precision agriculture: a review', Precision Agriculture, 13(6), pp. 693-712. 
Appendix 


\section{Appendix 1 - The its4land project}

its4land was a European Commission Horizon 2020 project that ran from 2016-2020. its4land aimed to deliver an innovative suite of land tenure recording tools that respond to sub-Saharan Africa's immense challenge to rapidly and cheaply map millions of unrecognized land rights in the region. its4land combined an innovation process with emerging geospatial technologies, including smart sketchmaps, UAVs, automated feature extraction, and geocloud services, to deliver land recording services that are end-user responsive, market-driven, and fit-for-purpose. The transdisciplinary work also developed supportive models for governance, capacity development, and business capitalization. Set in the East African development hotbeds of Rwanda, Kenya, and Ethiopia, its4land encapsulated three major phases that enabled contextualization, design, and eventual land sector transformation (see Figure A.1). This PhD research was embedded in the technical work package "Fly and Create" of the its4land project.

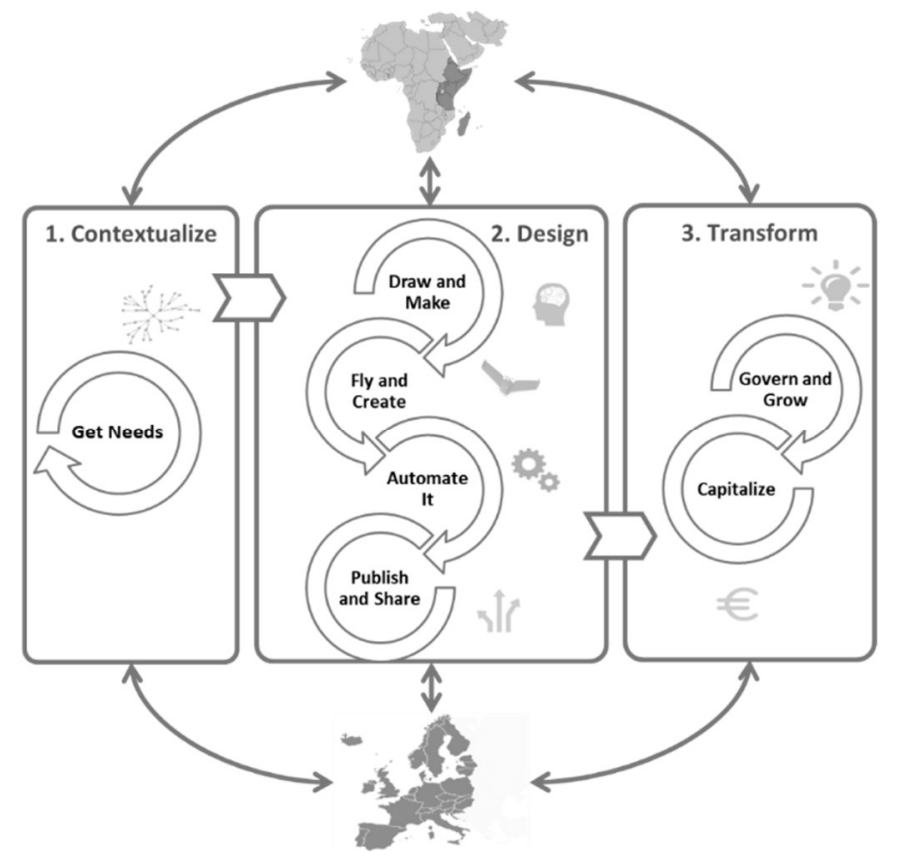

Figure A.1: Concept of the its4land project. 


\section{Appendix 2 - A practical excursus: Exemplifying a UAV-driven community mapping exercise to support the updating process of the Rwandan cadastre ${ }^{12}$}

This appendix exemplifies a UAV-driven participatory mapping approach and as an alternative concept to contemporary ground-based boundary surveying workflows and seeks to support the findings outlined in chapter 2 of this thesis. Data were collected in two main steps: 1) UAV data collection; 2) participatory mapping activity with the local citizen.

\section{Background}

The study area for the UAV data collection covers $3 \mathrm{~km}^{2}$ of the northern part of Ruhengeri Cell, District of Musanze, Northern Province of Rwanda. The area of interest was chosen due to significant urban developments during the past years (cf. Figure A.2). These changes are not visible in the aerial images from 2009 and are mainly also not updated in the Land Administration Information System (LAIS). Consequently, disputes arise as the current cadastre does not reflect the real situation on the ground, causing problems with updating mechanisms, correct compensations, and transactions. One of the villages in our study area was selected by Rwanda Land Management and Use Authority to conduct a systematic updating of the cadastre during the financial year 2019-2020. To show the potential of UAV technology, we chose this village to trial the community-based participatory mapping activity.

\section{The procedure of the mapping exercise}

The data is drawn from three main sources: 1) UAV data collection in February 2019, and 2) a participatory mapping activity with local residents, and 3) GNSS measurements of parcel boundaries. The evaluation of the opportunities provided by UAV technology for the updating process of the Rwandan cadastre is based on the geometric quality assessment and observations during the case study.

\footnotetext{
12 This appendix is based on:

Stöcker, C., Koeva, M. N., Nkerabigwi, P., \& Zevenbergen, J. A. (2020). UAV Technology: Opportunities to support the updating process of the Rwandan cadastre. In FIG Working Week 2020.
} 


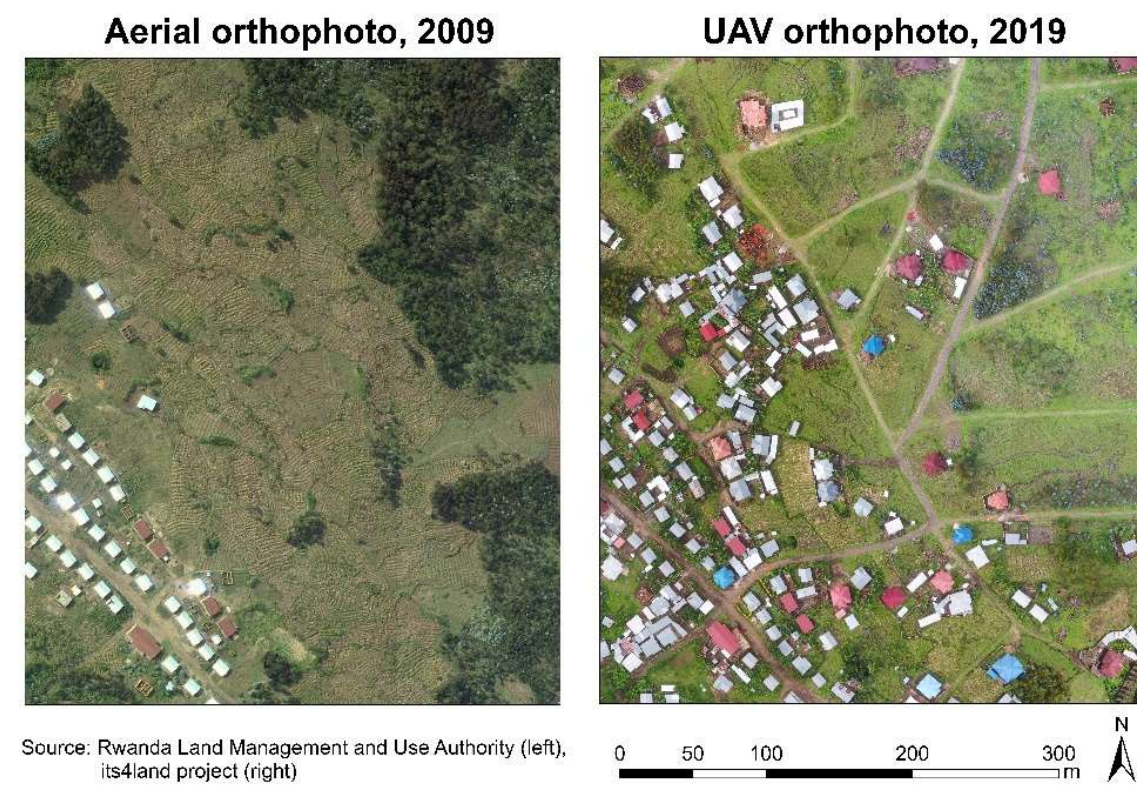

Figure A.2: Case study area for community-based participatory mapping

In collaboration with RLMUA, INES Ruhengeri and Esri Rwanda, we captured the area of interest with high-resolution images. The flights were carried out by the only licensed UAV company in Rwanda: Charis UAS Ltd., which holds all required licenses and permissions of the Rwanda Civil Aviation Authority to perform UAV flights. A DJI Inspire Pro UAV with an RGB sensor was employed to capture nadir pictures during the flight. The mission was planned and executed with Pix4D capture. Parameters were set according to the maximum allowed flight height of $120 \mathrm{~m}$ leading to a ground resolution of $2 \mathrm{~cm}$. Image overlap was set to $80 \%$ (forward) and $75 \%$ (side lap) to cater for unexpected wind turbulences and to ensure the creation of a reliable orthomosaic that is based on a strong image network. Additionally to the UAV data, we also collected ground truth data with a survey-grade GNSS (Trimble R8). For means of georeferencing, in total, 14 visible ground control points were marked throughout the study area. The points were deployed with spray paint and had a round shape with an identifiable centroid (Figure A.3). All locations were measured during two consecutive measurement campaigns with an accuracy below $2 \mathrm{~cm}$ as the GNSS devices were connected to the RTK network of the continuously operating reference system. The data was processed using the photogrammetric software Pix4D. Here, 8 points were used as ground control points within the photogrammetric processing. The remaining 6 points were used as independent checkpoints for quality control. 


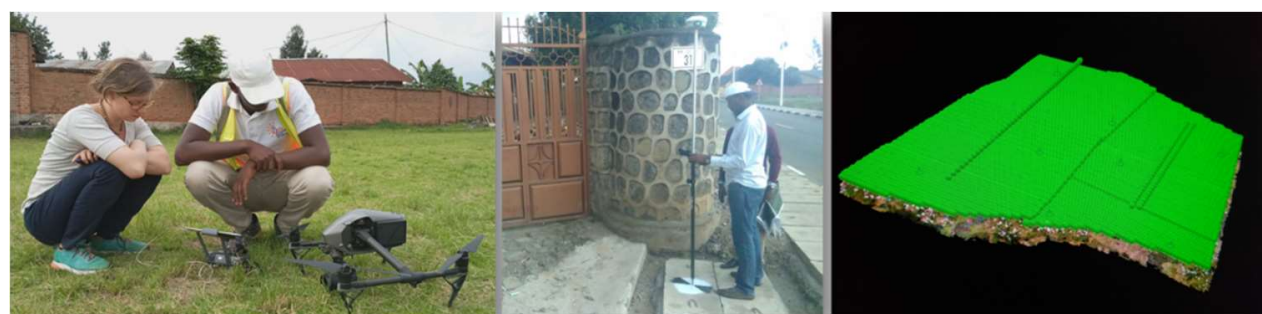

Figure A.3: UAV data collection. A: checking the UAV DJI Inspire Pro before the flight; $B$ : measurement of ground control points for georeferencing; $C$ : data processing in Pix4D

The second part of the data collection was focused on a participatory mapping activity to see how local inhabitants demarcate their land on the orthophoto. Furthermore, this mapping activity provided exciting insights into the ability of locals to identify their houses and boundaries. For this, we selected an area in Susa village, which is known to have land conflicts as well as unrecorded land transactions. Relevant local government stakeholders were notified and informed about the data collection. The UAV data of the respective area was processed during the weekend and printed with a scale of 1:300 on an A0 sheet (Figure A.4). The map was protected with a thin lamination layer, and waterproof markers were used for the drawing.

Accompanied by a village elder, we approached residents in their houses during the daytime on two consecutive days and asked if they could delineate their parcel boundary on the printed map. If the parcel was drawn successfully, we additionally collect information on the identification number of the parcel and the situation of ownership.
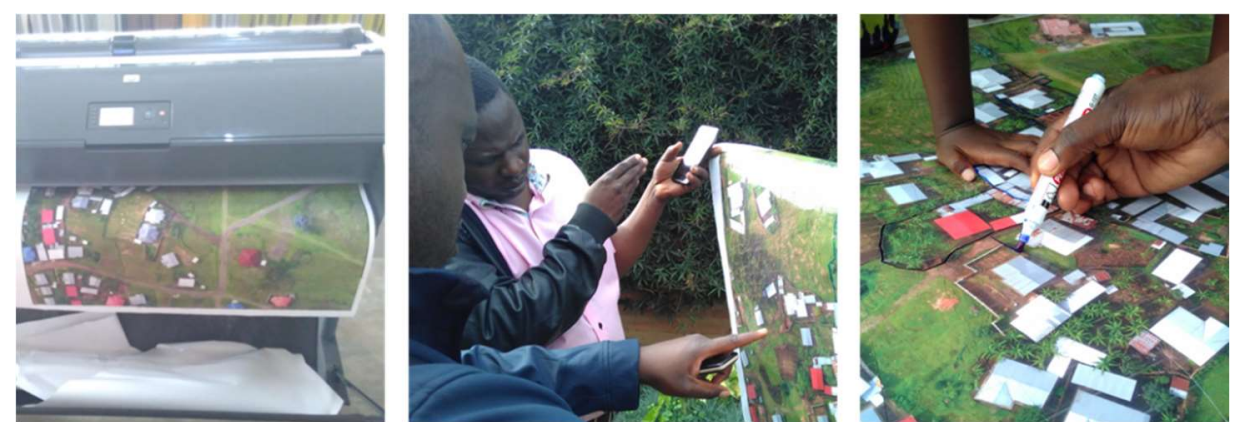

Figure A.4: Participatory mapping activity. Left: printing the orthophoto; Center: identifying houses on the orthophoto; Right: drawing the parcel boundary on the orthophoto

Additional GNSS measurements of nine selected parcels were carried out with a Trimble R8 GNSS device to allow independent quality control 
of the hand-drawn parcel outlines. Parcel corners were surveyed in RTK mode, with a baseline of approximately $2 \mathrm{~km}$. Measurements revealed a horizontal accuracy of less than $2 \mathrm{~cm}$. The following steps were accomplished to prepare the data for the quality assessment: 1) Scanning the AO paper with hand-drawn parcel outlines, 2) georeferencing the scanned map based on clearly identifiable landmarks, 3) digitizing parcel outlines by tracing hand-drawn lines, 4) measurement of Euclidian distances of corner points. The statistical comparison of point coordinates is graphically depicted in boxplots.

\section{Findings}

During the photogrammetric processing, three main data products can be derived from the UAV images. Firstly, a 3D point cloud is reconstructed, which presents a $3 \mathrm{D}$ visualization of the entire scene. As shown in Figure A.5, the surface, as well as rooftops, are represented consistently. Since the UAV only captured nadir images, the representation of vertical features such as walls of houses show lower point densities and are less consistent. Next to the 3D point cloud, a digital surface model (DSM) and the orthophoto can be derived. Even though all three datasets could be used to derive parcel information during a participatory mapping, the emphasis in this study was put on the orthophoto as this represents the dataset, which is the easiest to interpret for residents. The overall geometric accuracy of the orthophoto is $10.3 \mathrm{~cm}$, with a ground sampling distance of $2.1 \mathrm{~cm}$.
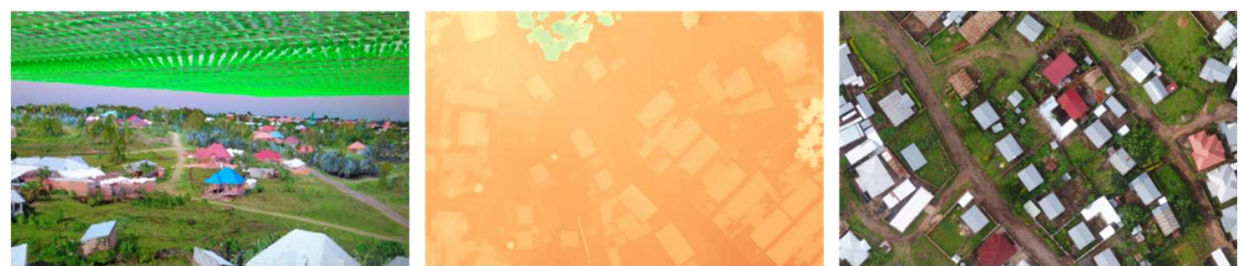

Figure A 5: Data products derived from UAV images. A: 3D point cloud; $B$ : digital surface model; C: Orthophoto

During the participatory mapping activity, 32 parcel boundaries were delineated by local residents. It was found that $72 \%$ of all people could identify their houses without or with little guidance. Landmarks such as construction works, a road, or special buildings that are known to everyone guided the orientation of local people. Furthermore, the high level of detail helped to draw the boundary as fences accurately, walls, special plants that usually demarcate the boundary, and even slight changes in the paving of streets were easy to detect. Few people refused to participate in the mapping activity as they reported landrelated conflicts. An overview of parcel outlines derived from the 
participatory mapping as well as information derived from the cadastral data in the LAIS is presented in Figure A.6.

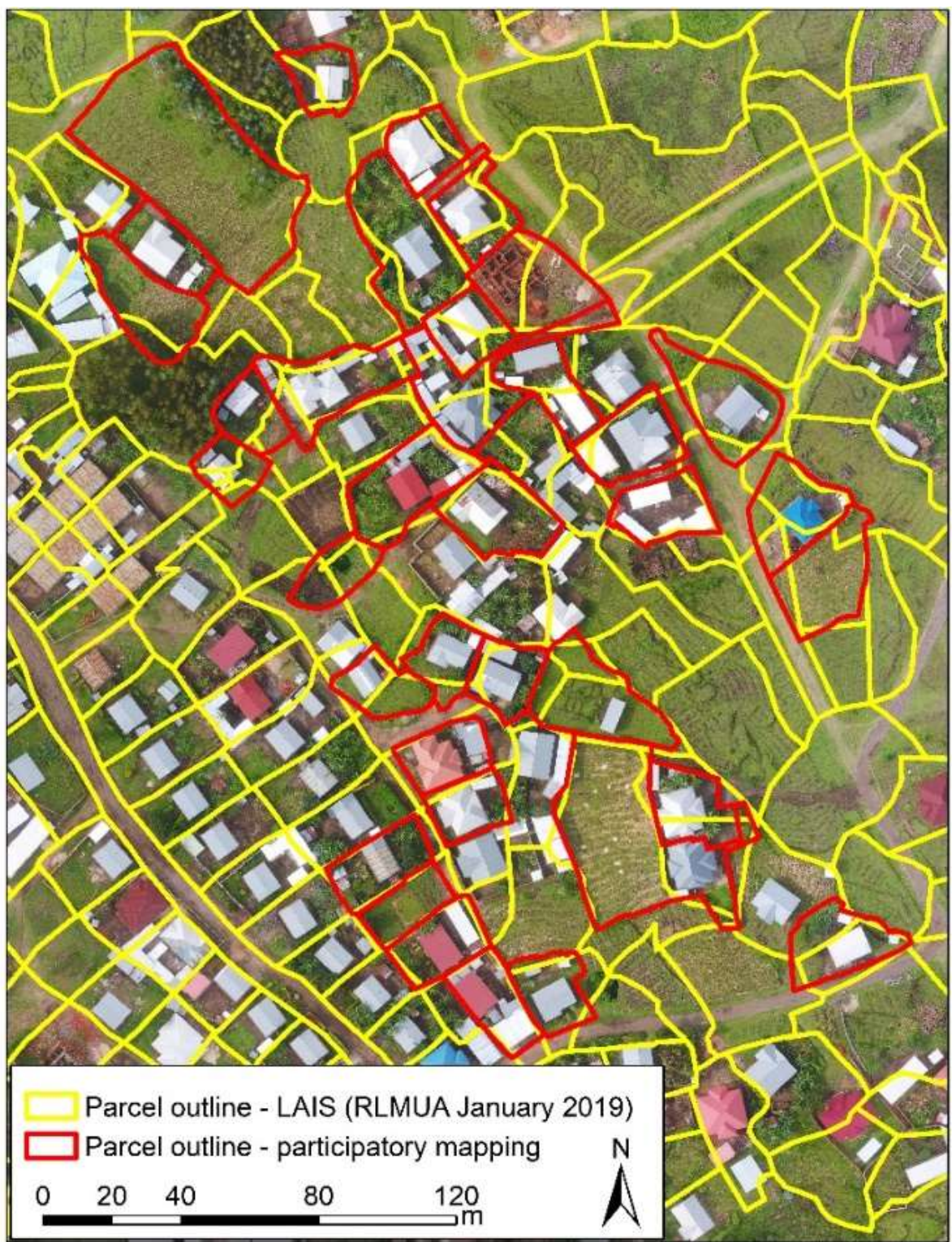

Figure A.6: Overview of parcel boundaries derived from participatory mapping (red) and cadastral database LAIS (yellow)

During data analysis, the parcel boundary drawn by the resident was linked to the existing parcel outline in the LAIS. The link was made through parcel IDs or via the parcel's location if the parcel ID was not known. One-third of all parcels could not directly be linked to an 
existing parcel as none of the conditions mentioned above was fulfilled. It is clearly visible that some parcels have the same extent in both datasets, especially for parcels with a regular rectangular pattern (lower left area in the map ). In other cases, two to three parcels from the participatory mapping activity form one parcel derived from the LAIS, which indicates that the land has not been officially subdivided yet. Lastly, in some instances, the drawn parcel boundary by local residents does not reflect the parcel outline from LAIS, neither in shape nor in size. This problem can be attributed to several issues: errors during first level registration in 2013, informal land transactions, or a faulty parcel survey during land transactions. In this specific case, especially the first level registration could be a potential source of errors as many developments took place during the period from 20092013, and the first level registration was carried out during 2012-2013, whereas the base maps were bound to the aerial image from 2009.

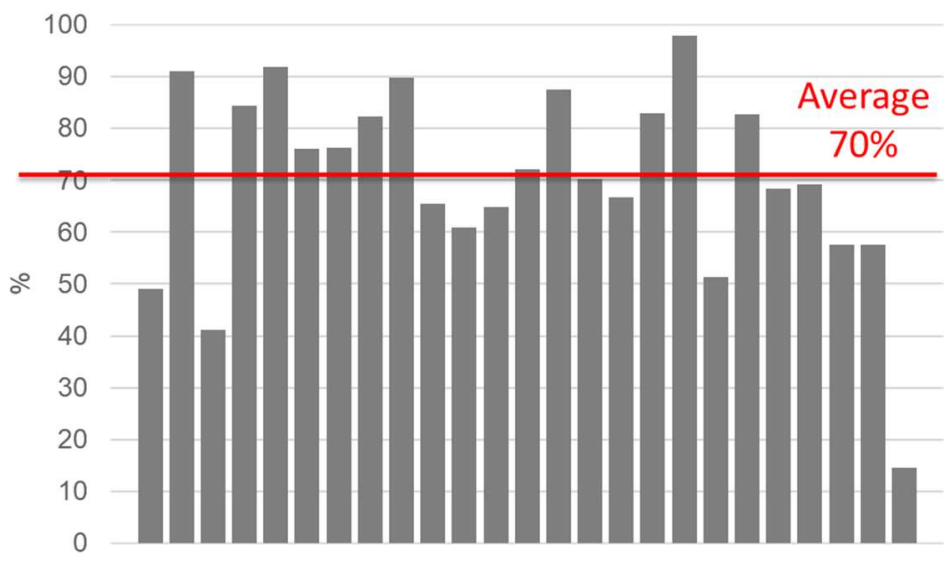

Figure A.7: Percentage of overlay from parcel area derived from participatory mapping with parcel area from LAIS

A closer analysis of the parcel shapes reveals that, on average, $70 \%$ of the dawn parcels overlay with the official parcel data in LAIS. From the diagram in Figure A.7, it can be seen that the range is very large and spreads from a minimum of $15 \%$ overlay to a maximum of $98 \%$ overlay. In this context, it should be noted that this average only refers to parcels that could be linked (25 out of 32), whereas the overall average might decrease when considering the "odd" parcels as well.

As the percentage of overlay alone does not provide the full picture of discrepancies in the spatial extent, we further compared the size of parcels. Here, negative values indicate that the drawn parcel is smaller than the parcel in LAIS, whereas positive values indicate that the drawn 
parcel is larger. The diagram in Figure A.8 shows two extremely negative values with more than $1000 \mathrm{~m}^{2}$ of land. Both parcels refer to a case in which the parcel size in LAIS is significantly larger as the land has been informally subdivided. The maximum value on the positive balance reflects a case where the owner has already bought his neighbour's property but did not report this transaction to the District. Besides those extreme deviations, all remaining differences are in a range of $+/-300 \mathrm{~m}^{2}$. Most of those deviations can probably not be explained by land transactions that are not yet processed but by an apparent discrepancy of the situation on the ground and the information in the cadastral database.

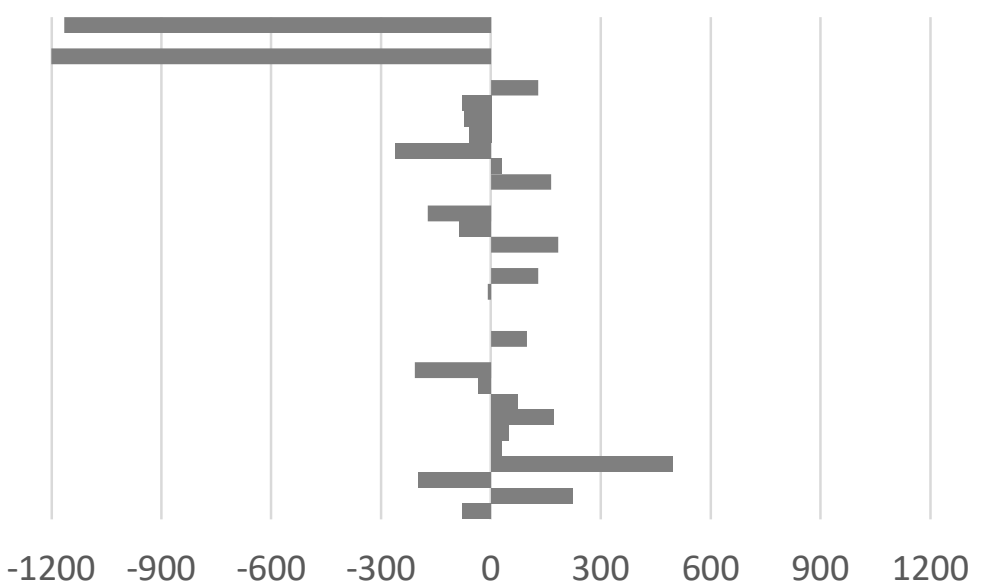

Figure A.8: Difference in parcel size $\left(\mathrm{m}^{2}\right)$. Negative values indicate that the drawn parcel is smaller than the parcel in LAIS, whereas positive values indicate that the drawn parcel is larger.

Since GNSS measurements and hand-drawn sketches were collected simultaneously and under the same condition, data from both survey methods can be directly compared. For means of a more transparent analysis, parcels have been distinguished according to their shape. Regularly shaped parcels refer to a standard geometric shape such as a rectangular or a circle, whereas irregularly shaped parcels include all other forms. Two main observations are visible in the statistical analysis (Figure A.9).

Firstly, significant geometric differences have been found for parcels with hardly any visible boundary. In both cases - regularly and irregularly shaped parcels - the maximum point distance of (more than) $5 \mathrm{~m}$ can be ascribed to parcel corners without clear landmarks such as walls or specific plants. Two irregularly shaped parcels are 
shown in the lower right of Figure A.9. Whereas the agricultural area with maize crops shows only minor discrepancies between differently surveyed parcel corners, the residential parcel has almost no visual features to determine the parcel boundary towards the North. Consequently, the GNSS point measurements and the hand-drawn parcel boundary do not represent the same spatial extent.

Secondly, irregularly shaped parcels show a significantly larger variance of point distances as represented by the interquartile range, thus tend to have a higher uncertainty in the representation of the hand-drawn outline. In contrast, both types of parcels show almost the same mean distance, with $1.30 \mathrm{~m}$ for regularly shaped parcels and $1.47 \mathrm{~m}$ for irregularly shaped parcels, respectively.
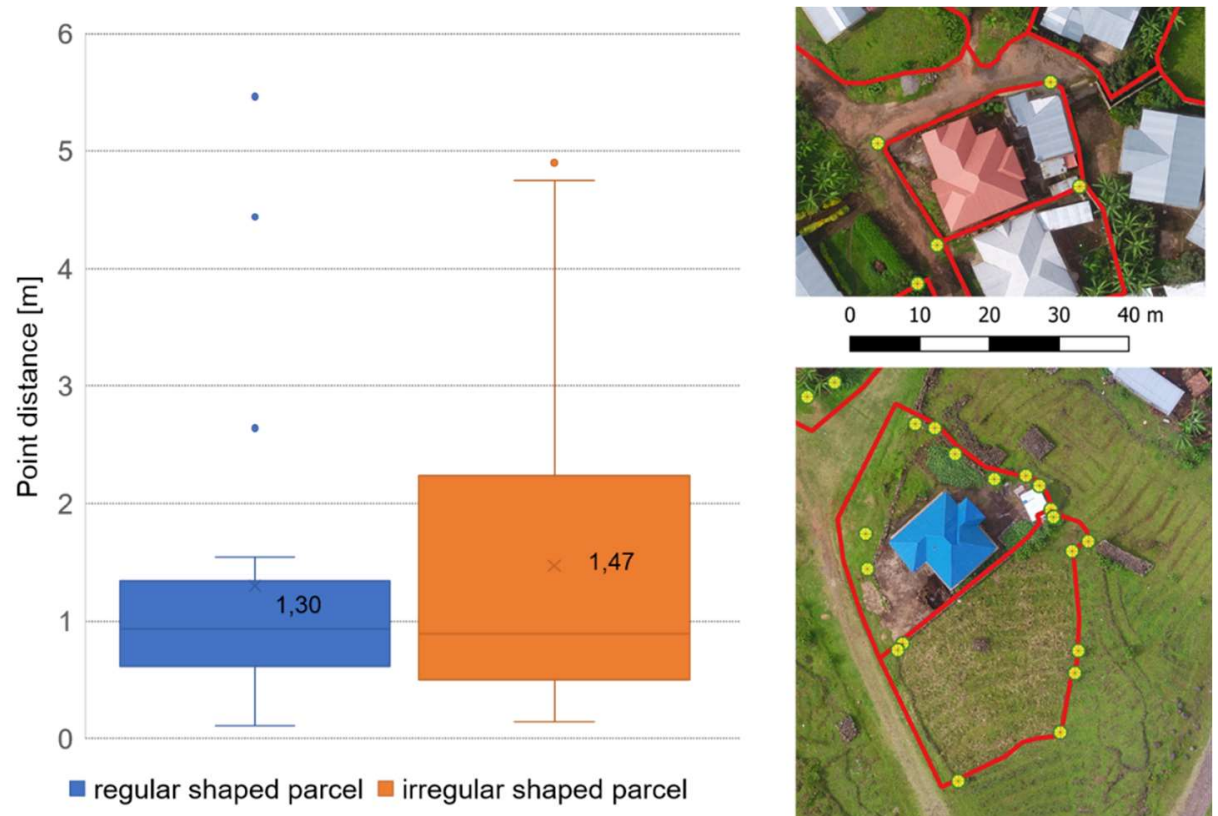

Figure A.9: Statistical analysis of distances of parcel corner points derived from GNSS measurements and participatory mapping (left), graphical examples for a regularly shaped parcel (upper right) and irregularly shaped parcel (lower right).

\section{Reflecting the practical excursus}

The Rwandan tax system is currently based on the areal extent of the parcel as well as the land use zone. As an example, landowners pay 40 RWF per $\mathrm{m}^{2}$ annually in a residential area. Deviations in the parcel extents derived from this study ultimately imply that landowners pay too little or too many taxes. This might lead to conflicts, especially when residents come in touch with the conventional system. Re- 
surveying and fixing existing parcel boundaries cause several problems. Firstly, almost all neighbouring parcels are affected by the survey and would require a re-survey as well. During fixing boundaries, surveyors are still using the old aerial images or google earth to validate and adjust the polygon of the geodetic survey in the field, not to raise concerns by the official land authorities. If the proposed cadastral parcel plan deviates too much from the original parcel, the re-survey might be rejected. Missing survey standards and a lack of well-trained professionals add to this problem and cumulate in a cadastral updating process, which is neither efficient nor reliable. Musanze is one of the fastest developing secondary cities in Rwanda, and land prices are increasing tremendously. In this regard, it will be a matter of time until conflicts during land transactions arise, especially when people pay the wrong amount of taxes or do not get compensated correctly due to the discrepancy of the LAIS and the reality on the ground.

Looking into the measurement accuracy of UAV-based participatory mapping of parcel boundaries, the accuracy assessment with GNSS measurements as reference data revealed a mean offset of $1.3 \mathrm{~m}$ for regularly shaped parcels and $1.47 \mathrm{~m}$ for irregularly shaped parcels. The geometric difference in point locations has various error sources. Both surveying methods contain different levels of accuracy. Whereas the mode of ambiguity resolution determines the accuracy of the GNSS measurement, the UAV-based participatory mapping approach shows various sources of errors. Firstly, the error of the photogrammetric reconstruction, and secondly, the error of the drawing, which can be subject to map scale, the thickness of the pencil, as well as the ability of the local citizen to correctly determine and draw the parcel boundary. Aside from extreme outliers in the range from 4-5m point distance, the results in this study suggest a measurement accuracy of $1 \mathrm{~m}$ to $2 \mathrm{~m}$ for UAV-based participatory mapping of parcel boundaries when following the approach described here.

Despite the geometric measurement accuracy, we could validate that people can understand the map and identify their houses, primarily due to the high resolution and clear visualization of small features such as walls, surface characteristics of roads, and even particular forms of vegetation. The immediateness of the data delivery of only a few days from the UAV data collection and the printout of the map certainly helped in this procedure as we observed that people are more likely to identify small features such as little piles of sand or stones that they are used to see in their every-day life. The high level of detail further reduced disputes about the location of boundaries to a minimum. 
Although we went from house to house and did not include all neighbours during the boundary delineation process, not even one party disputed the line drawn by its neighbour.

Even though the discrepancies of the LAIS and the hand-drawn parcel outlines cannot be solely ascribed to one or another reason, it could be shown that UAV orthophotos can help to detect informal land transactions. Secondly, significant boundary offsets from the first registration can be spotted, especially when parcel boundaries are crossing houses and are not aligned to any visible boundaries on the ground. At a lower level of implementation, UAV data could further be used by the District government to validate geodetic surveys of professionals. Referring back to the situation that some regions in Rwanda were nominated for a systematic re-survey, UAVs would be a suitable technology to provide an up-to-date base map for regions whose extent is limited to a few $\mathrm{km}^{2}$.

\section{Conclusions}

The results of this practical excursus have shown that the current LAIS data shows significant discrepancies from the real situation on the ground in the study area. The case study showed that UAV-based upto-date base data could significantly improve current surveying practices either for validation or even as a primary data source for participatory mapping activities to determine general boundaries with an overall accuracy of $1-2 \mathrm{~m}$. The suggested workflow shows strong benefits compared to contemporary ground-based surveying, particularly in terms of transparency of the data collection and the participation of residents. Especially the task of systematic resurveying of small-to-medium scale areas should be considered to employ UAV technology as a fit-for-purpose (Enemark et al., 2014) mapping and surveying practice. 
Appendix 3 - Overview of UAV regulations in selected countries 


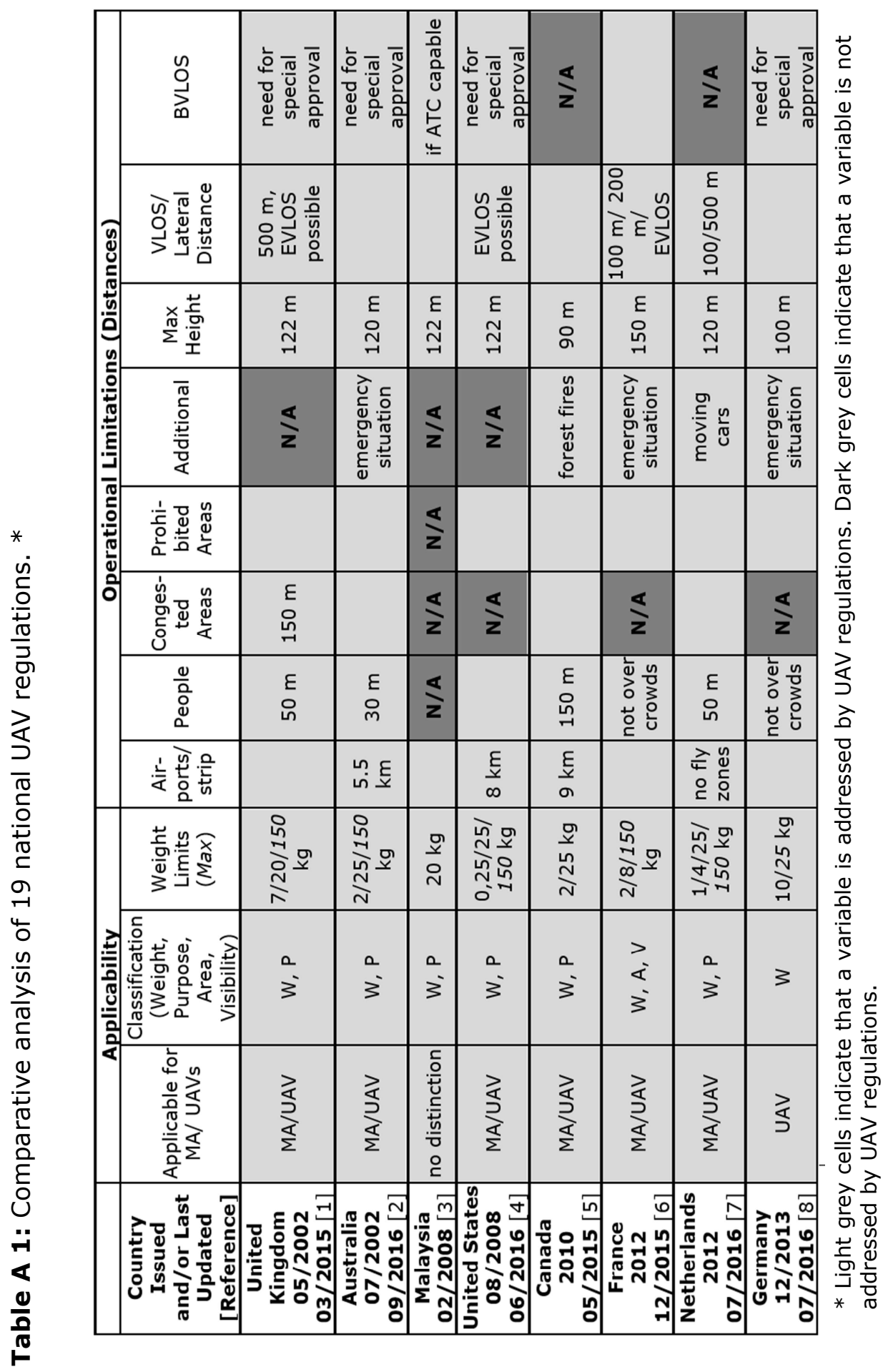




\begin{tabular}{|c|c|c|c|c|c|c|c|c|c|c|c|c|}
\hline & $\stackrel{0}{0}_{0}^{n}$ & 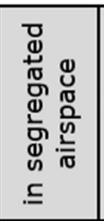 & 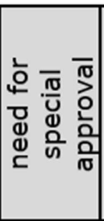 & 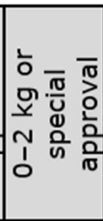 & 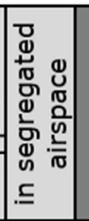 & 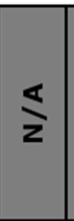 & $\frac{\varsigma}{z}$ & 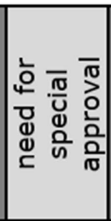 & $\leqslant$ & & & $\leqslant$ \\
\hline y & 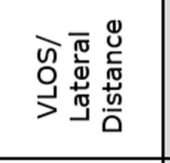 & है & & 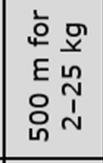 & & $\begin{array}{l}E \\
\text { in }\end{array}$ & \begin{tabular}{|l|}
$E$ \\
员 \\
\end{tabular} & 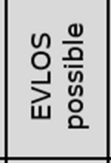 & & & $\begin{array}{l}E \\
\stackrel{0}{\circ}\end{array}$ & \\
\hline$\underline{\theta}$ & 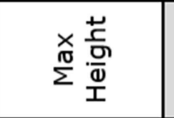 & $\begin{array}{l}E \\
\text { E } \\
\text { in }\end{array}$ & $\begin{array}{l}E \\
\text { L } \\
\text { 음 }\end{array}$ & $\begin{array}{l}\varepsilon \\
\text { ָे } \\
\text { Tे }\end{array}$ & $\begin{array}{l}E \\
N \\
\mathbb{F}\end{array}$ & \begin{tabular}{l|}
$E$ \\
$D$ \\
$\stackrel{M}{-}$ \\
-1
\end{tabular} & \begin{tabular}{l|l}
$E$ \\
$N$ \\
$N$ \\
\\
\end{tabular} & $\begin{array}{l}E \\
\mathbb{N}\end{array}$ & 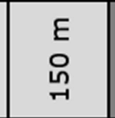 & $\leqslant$ & \begin{tabular}{l|l|} 
& \\
8 & \\
\end{tabular} & $\leqslant$ \\
\hline & 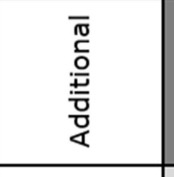 & $\leqslant$ & $\frac{\Sigma}{z}$ & $\leqslant$ & $\overleftarrow{z}$ & $\begin{array}{l}\stackrel{c}{\bar{E}} \\
\stackrel{\circ}{o} \\
\mathrm{v}\end{array}$ & $\mid$ & $\overleftarrow{z}$ & $\frac{\varsigma}{z}$ & 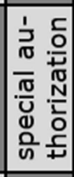 & $\leqslant$ & 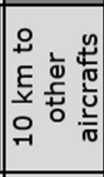 \\
\hline & 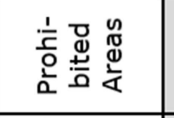 & & & & & & & & & $\frac{\varsigma}{z}$ & & \\
\hline 0 & 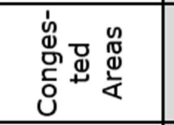 & $\begin{array}{l}E \\
\text { in } \\
\text { ñ }\end{array}$ & $\frac{\varangle}{z}$ & & 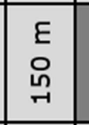 & $\frac{\varsigma}{z}$ & & & & $\leqslant$ & & \\
\hline & $\begin{array}{l}\frac{\omega}{0} \\
0 \\
0 \\
0\end{array}$ & $\begin{array}{l}E \\
\text { in }\end{array}$ & 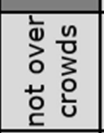 & 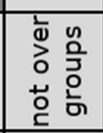 & $\begin{array}{l}E \\
\text { in }\end{array}$ & $\begin{array}{l}E \\
\stackrel{m}{m}\end{array}$ & & $\begin{array}{l}E \\
\text { in }\end{array}$ & $\begin{array}{l}E \\
\text { O }\end{array}$ & $\frac{\varsigma}{z}$ & $\begin{array}{l}E \\
\text { in }\end{array}$ & $\leqslant$ \\
\hline & 完产高 & $\frac{\text { E }}{\text { in }}$ & & $\stackrel{n}{\infty}_{\infty}^{n} \underline{\varepsilon}$ & & $\frac{E}{N}$ & \begin{tabular}{l|}
$\frac{E}{y}$ \\
in
\end{tabular} & 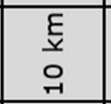 & 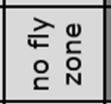 & $\frac{\pi}{z}$ & 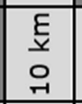 & \\
\hline & 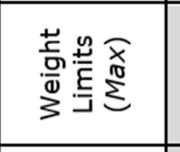 & $\begin{array}{ll}\frac{n}{n} \\
\frac{n}{4} \\
\end{array}$ & 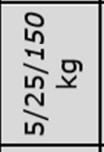 & $\begin{array}{l}\stackrel{n}{n} \\
\stackrel{n}{n} g \\
\stackrel{n}{N} \\
\end{array}$ & 柋 & $\begin{array}{l}\frac{8}{8} \\
0\end{array}$ & $\begin{array}{l}\mathscr{g} \\
\stackrel{2}{N} \\
\stackrel{n}{N}\end{array}$ & $\begin{array}{l}\frac{9}{2} \\
\stackrel{N}{n} \\
\end{array}$ & $\leqslant$ & $\frac{\leqslant}{z}$ & 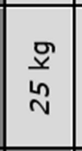 & 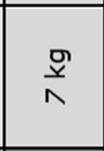 \\
\hline & 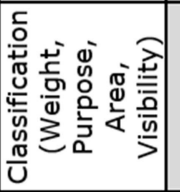 & $\begin{array}{l}4 \\
3\end{array}$ & $\$$ & 3 & 3 & 3 & 3 & $\begin{array}{l}> \\
3\end{array}$ & $\leqslant$ & $\frac{\varsigma}{z}$ & $\leqslant$ & $\frac{\varangle}{z}$ \\
\hline & 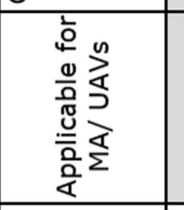 & \্ব & 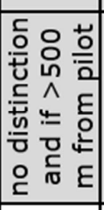 & 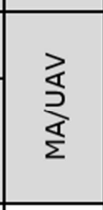 & 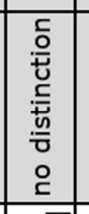 & 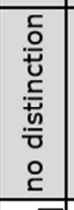 & & 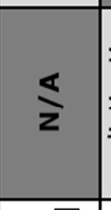 & 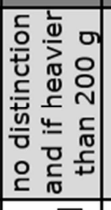 & 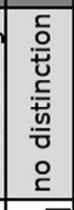 & 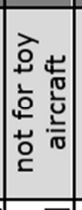 & 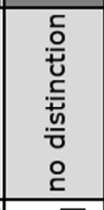 \\
\hline & 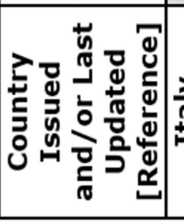 & 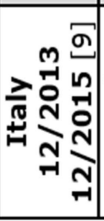 & 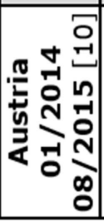 & 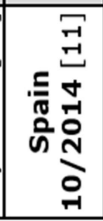 & 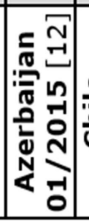 & 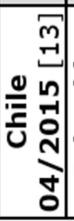 & 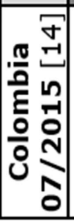 & 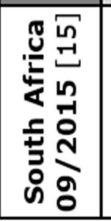 & 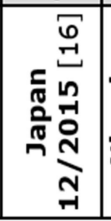 & | & 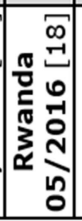 & 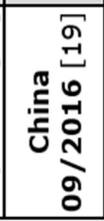 \\
\hline
\end{tabular}




\begin{tabular}{|c|c|c|c|c|c|c|c|c|c|}
\hline \multirow{2}{*}{ 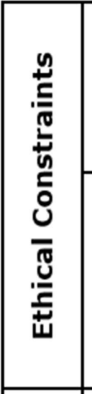 } & 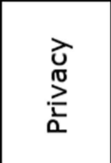 & 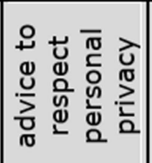 & \multirow{2}{*}{ 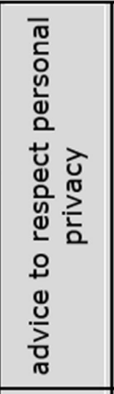 } & \multirow{2}{*}{ 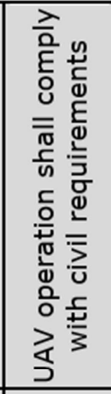 } & \multirow{2}{*}{ 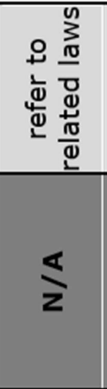 } & \multirow{2}{*}{ 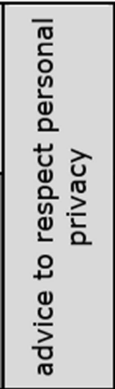 } & 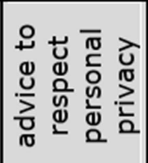 & \multirow{2}{*}{ 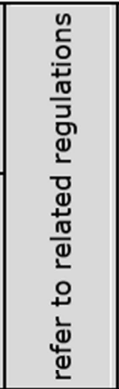 } & \multirow{2}{*}{ 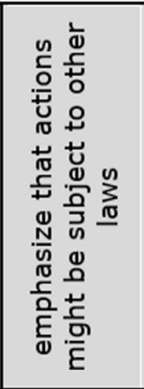 } \\
\hline & 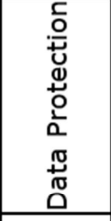 & 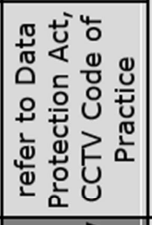 & & & & & 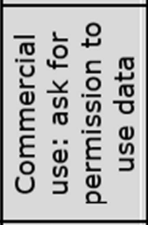 & & \\
\hline 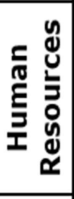 & 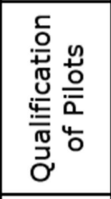 & 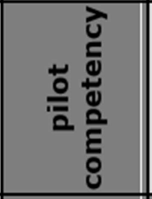 & 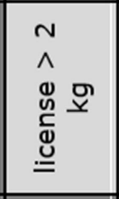 & 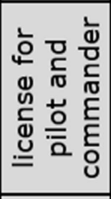 & 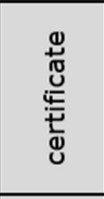 & 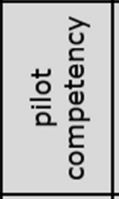 & 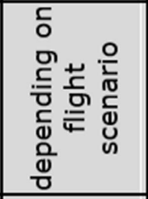 & 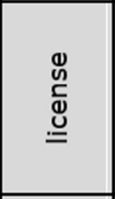 & 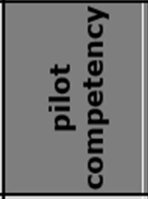 \\
\hline \multirow{3}{*}{ 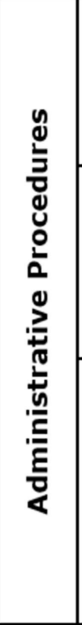 } & 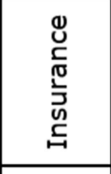 & $\leqslant$ & 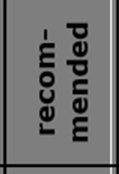 & & 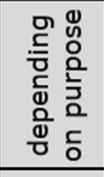 & 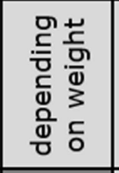 & & & \\
\hline & 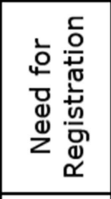 & $\frac{\leqslant}{z}$ & $\leqslant$ & $\begin{array}{l}\text { v } \\
\text { ì } \\
\end{array}$ & 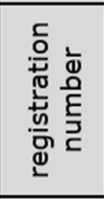 & $\leqslant$ & 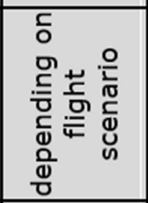 & & $\frac{\ll}{z}$ \\
\hline & 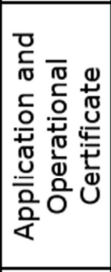 & 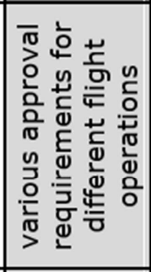 & 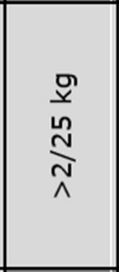 & 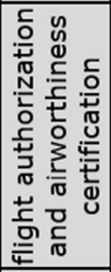 & 敢 & 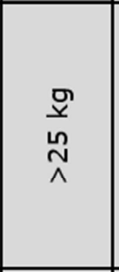 & 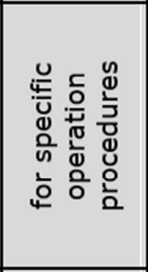 & 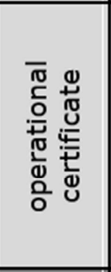 & 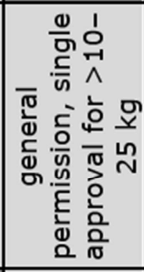 \\
\hline \multirow{2}{*}{ 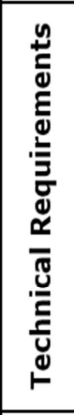 } & 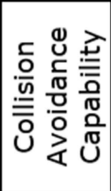 & 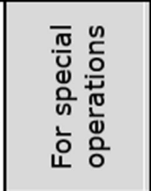 & $\leqslant$ & \multirow{2}{*}{ 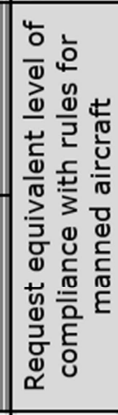 } & $\frac{\leqslant}{z}$ & $\begin{array}{l}\stackrel{0}{\vec{v}} \\
\stackrel{n}{N} \\
\wedge\end{array}$ & 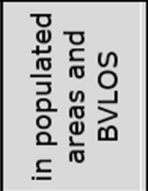 & $\leqslant$ & 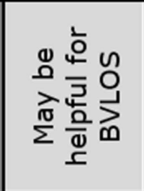 \\
\hline & 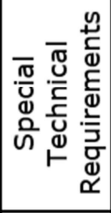 & & $\leqslant$ & & $\bar{z}$ & $\hat{z}$ & g) & 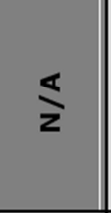 & $\begin{array}{l}\text { वे } \\
\text { वे } \\
\text { ते } \\
\wedge\end{array}$ \\
\hline & 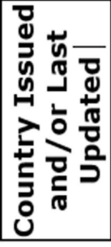 & 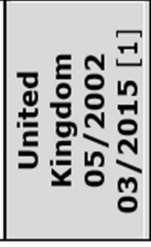 & 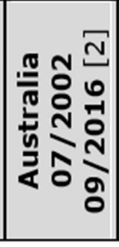 & 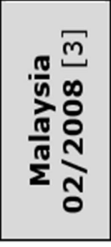 & 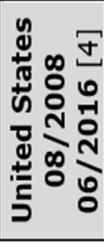 & 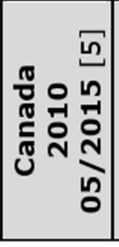 & 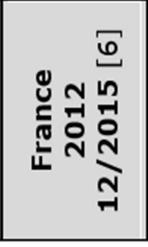 & 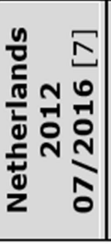 & 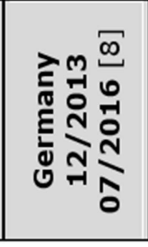 \\
\hline
\end{tabular}




\begin{tabular}{|c|c|c|c|c|c|c|c|c|}
\hline \multirow{2}{*}{ 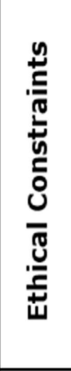 } & 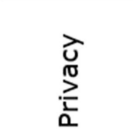 & $\overleftarrow{z}$ & $\frac{\varsigma}{z}$ & $\frac{s}{z}$ & $\frac{\ll}{z}$ & $\frac{\ll}{z}$ & \multirow{2}{*}{ 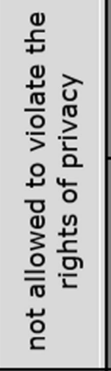 } & \multirow{2}{*}{$\frac{\frac{s}{2}}{\frac{1}{2}}$} \\
\hline & 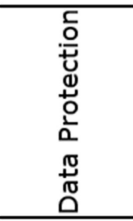 & 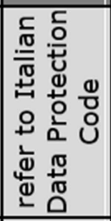 & $\frac{\S}{z}$ & $\frac{\leqslant}{z}$ & $\leqslant$ & $\frac{\leqslant}{z}$ & & \\
\hline 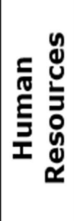 & 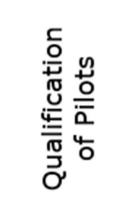 & 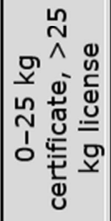 & 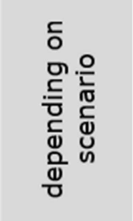 & 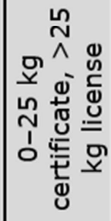 & 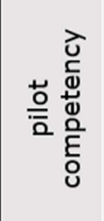 & 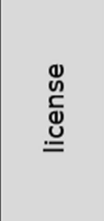 & 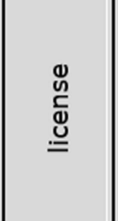 & 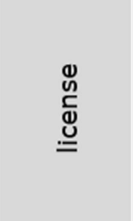 \\
\hline \multirow{3}{*}{ 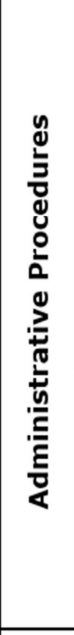 } & 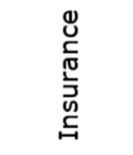 & & & & & $\leqslant$ & & \\
\hline & 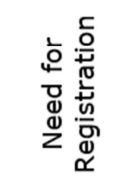 & 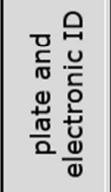 & 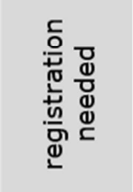 & 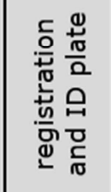 & $\begin{array}{l}\text { g } \\
\stackrel{2}{N} \\
\text { N }\end{array}$ & & & 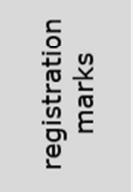 \\
\hline & 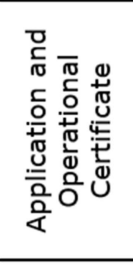 & 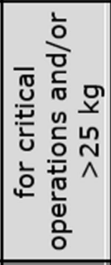 & 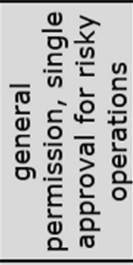 & 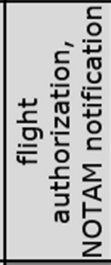 & 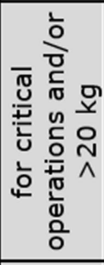 & 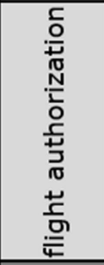 & 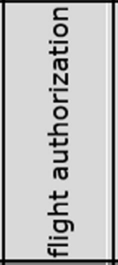 & 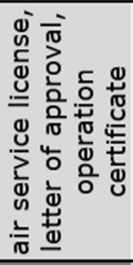 \\
\hline \multirow{3}{*}{ 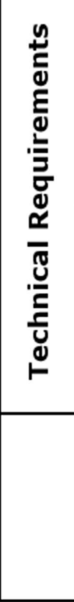 } & 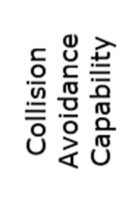 & $\overleftarrow{z}$ & 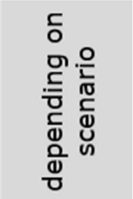 & $\leqslant$ & 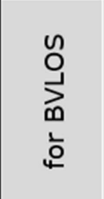 & $\leqslant$ & $\leqslant$ & $\leqslant$ \\
\hline & 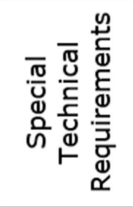 & 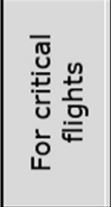 & 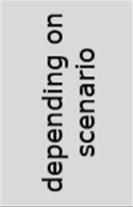 & $\leqslant$ & $\overleftarrow{z}$ & 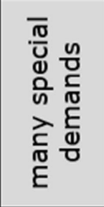 & 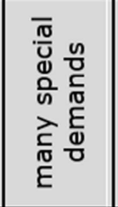 & \\
\hline & 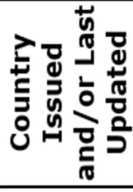 & ) & 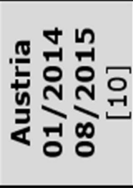 & 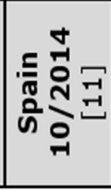 & 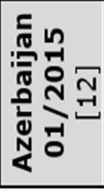 & 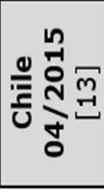 & 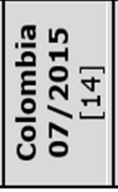 & 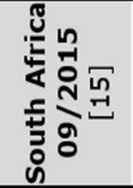 \\
\hline
\end{tabular}




\begin{tabular}{|c|c|c|c|c|c|}
\hline \multirow{2}{*}{ 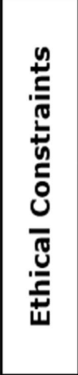 } & 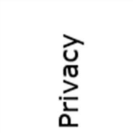 & $\frac{s}{z}$ & $\overleftarrow{z}$ & \multirow{2}{*}{ 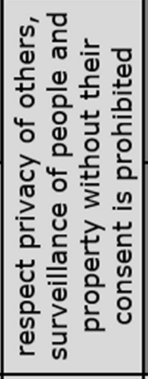 } & \multirow{2}{*}{$\frac{\frac{s}{2}}{\frac{2}{2}}$} \\
\hline & 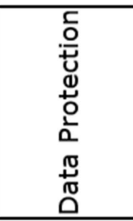 & $\frac{\Sigma}{z}$ & 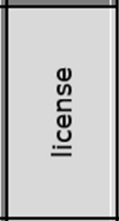 & & \\
\hline 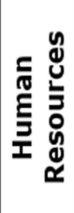 & 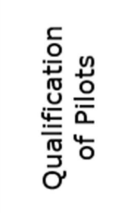 & $\leqslant$ & 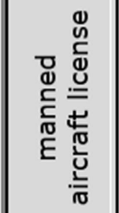 & 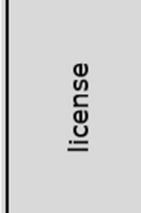 & 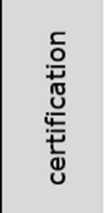 \\
\hline \multirow{3}{*}{ 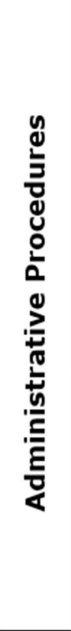 } & 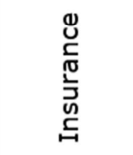 & $\leqslant$ & $\leqslant$ & & $\frac{\leqslant}{z}$ \\
\hline & 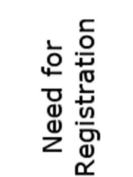 & $\leqslant$ & $\leqslant$ & 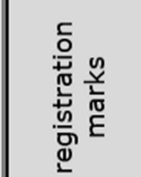 & 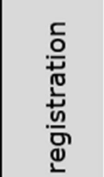 \\
\hline & 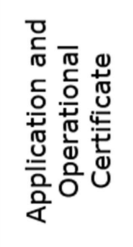 & 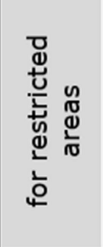 & 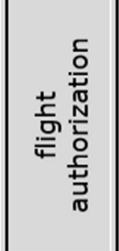 & 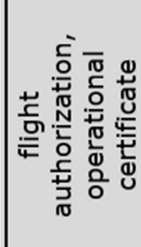 & 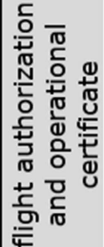 \\
\hline \multirow{3}{*}{ 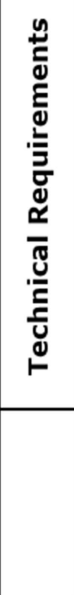 } & 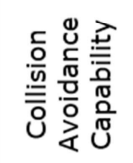 & $\leqslant$ & $\frac{\varangle}{z}$ & $\leqslant$ & $\frac{\varsigma}{z}$ \\
\hline & 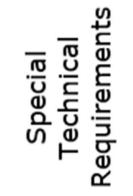 & $\leqslant$ & $\leqslant$ & $\leqslant$ & $\frac{\leqslant}{z}$ \\
\hline & 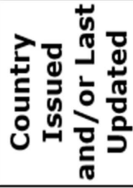 & 践 & 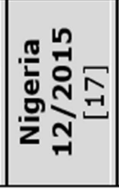 & 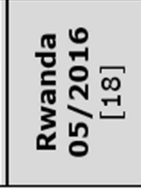 & 产 \\
\hline
\end{tabular}




\section{References of national UAV regulations:}

1. United Kingdom: Civil Aviation Authority UK. Unmanned Aircraft System Operations in UK Airspace-Guidance; 2015. Available online: https://publicapps.caa.co.uk/docs/33/CAP\%20722\%20Sixth\%20Edition $\% 20$ March\% 202015.pdf (accessed on 15 October 2016).

2. Australia: CASA Advisory Cirular AC 101-10. Remotely Piloted Aircraft Systems-Operation of Excluded RPA ( Other than Model Aircraft); 2016. Available online: https://www.casa.gov.au/files/ac10110pdf (accessed on 15 February 2017).

3. Malaysia: Aeronautical Information Services Malaysia. Unmanned Aerial Vehicle (UAV) Operations in Malaysian Airspace; 2008. Available online: http://aip.dca.gov.my/aip\%20pdf\%20new/AIC/AIC\%20200804.pdf (accessed on 15 February 2017).

4. United States: U.S. Department of Transportation Federal Aviation Administration. Advisory Circular Small Unmanned Aircraft Systems (sUAS); 2016. Available online: https://www.faa.gov/regulations_policies/advisory_circulars/index.cfm/g o/document.list/parentTopicID/137 (accessed on 15 October 2016).

5. Canada: Transport Canada Notice of Proposed Amendment Unmanned Air Vehicles; 2015. Available online: http://wwwapps.tc.gc.ca/Saf-SecSur/2/NPA-APM/doc.aspx?id=10294 (accessed on 15 February 2017).

6. France: Diretion Generale de I'Aviation Civile Guide DSAC - Aeronefs Circulant Sans Personne a bord: Activites Particulieres; 2016.

Available online: http://www.developpementdurable.gouv.fr/sites/default/files/Guide\%20Activités\%20particulières\% 20v1.2\%2010jan17.pdf (accessed on 15 February 2017).

7. The Netherlands: ILT Staatscourant Nr. 27761; 2016. Available online: https://www.ilent.nl/onderwerpen/transport/luchtvaart/dronevliegers/ (accessed on 15.02.2017).

8. Germany: Deutsche Flugsicherung. Nachrichten fuer Luftfahrer; 2016. Available online: https://www.dfs.de/dfs_homepage/de/Unternehmen/Richtlinien/1-78616.pdf (accessed on 15 February 2017).

Italy: L'Ente Nazionale per I'Aviazione Civile. Regulation Remotely Piloted Aerial Vehicles; 2015. Available online:

https://www.enac.gov.it/La_Normativa/Normativa_Enac/Regolamenti/Re golamenti_ad_hoc/info-122671512.html (accessed on 15.02.2017).

9. Austria: austro control Lufttüchtigkeits- und Betriebstüchtigkeitsanforderungen für unbemannte Luftfahrzeuge der Klasse 1; 2015. Available online: 
http://www.ris.bka.gv.at/Dokument.wxe?Abfrage=Bundesnormen\&Doku mentnummer=NOR40151655 (accessed on 15 February 2017).

10. Spain: Ministerio De Agricultura Boletín oficial del estado Aeronaves civiles pilotadas por control remoto; 2014. Available online: http://www.boe.es/boe/dias/2014/10/17/pdfs/BOE-A-2014-10517.pdf (accessed on 18 March 2017).

11. Azerbaijan: Republic of Azerbaijan State Civil Aviation Administration. Unmanned Aircraft Operations; 2015.

Available online:

http://www.caa.gov.az/index.php?option=com_k2\&view=item\&id $=297: u$ nmanned-aircraft-operations\&Itemid=174\&lang=en (accessed on 15 February 2017).

12. Chile: Direccion General De Aeronautica Chile Dan 151-Operaciones de Aeronaves Pilotadas a Distancia (RPAS).

Available online: https://www.dgac.gob.cl/portalweb/restportalweb/jcr/repository/collaboration/sites\%20content/live/dgac/catego ries/normativas/normasDAN/documents/DAN_151-20150413.pdf (accessed on 15 February 2017).

13. Colombia: Aeronautica Civil de Colombia Requisitos Generales de Aeronavegabilidad y Operaciones para RPAS; 2015. Available online: http://www.aerocivil.gov.co/autoridad-de-la-aviacion-civil/certificacionylicenciamiento/Documents/CIRCULAR\%20REGLAMENTARIA $\% 20 \% 2000$ 2\%20-\%20RPAS.pdf\#search=RPAS (accessed on 15 February 2017).

14. South Africa: South African Civil Aviation Authority Technical guidance material for RPAS part 101. Available online: http://www.caa.co.za/Pages/RPAS/Remotely\%20Piloted\%20Aircraft $\% 20$ Systems.aspx (accessed on 28 March 2017).

15. Japan: Japan Ministry of Land Infrastructure Transport and Tourism Amendments to the Aeronautical Act. Available online: http://www.mlit.go.jp/en/koku/uas.html (accessed on 15 February 2017)

16. Nigeria: Federal Republic of Nigeria. Nigeria Civil Aviation Regulation. Available online: http://ncaa.gov.ng/ncaa-issues-safety-guidelines-fordrone-operators/ (accessed on 15 February 2017)

17. Rwanda: Rwanda Civil Aviation Authority Ministerial Regulations No. 01/MOS/Trans/016 of 26/04/2016 Relating to Unmanned Civil Aircraft System. Available online: (accessed on 15 February 2017)

18. China: Civil Aviation Administration of China Measures for the Administration of Air Traffic in Civil Unmanned Aircraft Systems. Available online:

http://www.caac.gov.cn/XXGK/XXGK/GFXWJ/201610/P02016100834566 8760913.pdf (accessed on 15 February 2017) 


\section{Summary}

Secure land rights promote tenure security, a critical factor to fight hunger and poverty, the promotion of peace, economic growth, and the sustainable use of the environment. However, a large share of the global population feels insecure about their property rights, mainly attributed to missing documentation. During the past years, geospatial innovations and alterative concepts of land tenure recording disrupt traditional means of cadastral mapping and land administration. One of these innovations refers to Unmanned Aerial Vehicles (UAVs) offering flexible, cheap, and real-time acquisition of high resolution aerial imagery. Characteristics, which are considered key for addressing current land administration challenges.

Although reported benefits seem to be auspicious, the adoption of UAVs as an innovative tool for cadastral mapping remains remarkably poor. Previous works mainly refer to data-driven and technological assets of the utilization of UAVs and do not consider societal, governance and institutional settings and needs of people and groups. To respond to this gap this thesis utilizes a socio-technical approach to explore UAV-based data acquisition workflows as a tool for responsible land administration. Moreover, this thesis emphasizes on the interrelationship of three key system elements: technology (UAVs), processes (land administration processes) and actors (stakeholders). These interrelationships are reflected in four specific research objectives. Being embedded in the EU H2O2O funded international research and innovation project its4land, parts of this thesis were exemplified in the East African context, including test flights, workshops, and mapping activities.

The first part assesses the capabilities of UAV technology to match the needs of land professionals in Rwanda. A needs assessment enabled the expression of a range of land information needs across multiple levels and stakeholder sectors. Although ranked differently, the need for high-resolution, up-to-date land information was consistently identified in the final lists of all group discussions. Test flights with multiple UAVs and data processing in Rwanda proved, that UAVs are able to provide valuable spatial information for various stakeholder. However, the examination also suggests four main aspects which may reveal challenges to UAV interventions to derive land information in the context of Rwanda: 1) the hilly terrain, 2) UAV regulations, 3) ground-truthing in urban areas, 4) data processing capacities at the national land administration authority. In sum, although UAVs can play a key role in satisfying stakeholder needs, 
structural and capacity conditions currently present major challenges to exploit the potential of UAV data acquisition workflows.

Triggered by tremendous regulatory obstacles to fly UAVs in Eastern Africa, the second part of this thesis analyses the interface between UAV technology and stakeholders in terms of legal and regulatory frameworks. To get a better understanding of past, present and future developments of UAV regulations, a thorough literature review and a comparative analysis of national UAV regulations was conducted. Although all UAV regulations have a similar target - minimizing the risk for people and property on the ground - a distinct heterogeneity of national regulatory frameworks was investigated. However, commonalities such as mandatory platform registration, obligatory insurance coverage and standard pilot licensing procedures indicate trends towards mature national UAV regulation. Desirable trends are seen in the coexistence of hard and soft regulations and the successful international dialogue that may eventually provide a legal framework for harmonized regulatory standards.

The third part of this thesis explores the technical and operational aspect of UAV workflows and assesses different data quality measures with the aim to recommend optimal UAV data acquisition workflows. Imagery from six study areas across Europe and Africa, representing overall more than 100 different flight configurations, provided the basis for an integrated data quality assessment including various aspects of the photogrammetric processing chain. The results suggest that scene context, flight configuration, and GCP setup significantly impact the final data quality and subsequent automatic delineation of visual cadastral boundaries. Moreover, even though the root mean square error of checkpoint residuals as a commonly accepted error measure lies within a range of few centimeters in all datasets, our results show large discrepancies of the accuracy and the completeness of automatically detected cadastral features for orthophotos generated from different flight plans. With its unique combination of methods and integration of various study sites, these results and recommendations can help land professionals and bottom-up initiatives alike to optimize existing and future UAV data collection workflows.

Despite the prospects and market opportunities in land administration, there is a gap between experimentation and widespread diffusion of UAV technology. To understand the dynamics of the diffusion process of UAV technology as a tool for effective land 
administration, the fourth part of this thesis integrates the Framework for Effective Land Administration (FELA) and the Hype Cycle concept. The majority of experts estimated UAV technology to be in a phase in which the innovation needs to overcome initial unmet expectations to foster market development and increased adoption. The results indicate a changing importance of different FELA pathways during this process, led by alternating top-down and bottom-up dynamics. Enabling laws and policies in addition to supporting governance, accountability and institutions are found to be crucial to create a UAV-friendly national ecosystem early on in the process of technology adoption and allay exaggerated expectations. Once this ecosystem has been created, market demand is expected to surge driven by partnerships, adapted standards, tech advocacy and awareness-raising campaigns, highlighting the superiority of highresolution data amongst other benefits of UAV technology. These insights can be used as a baseline to direct national strategic decisions towards the increased adoption of UAVs in land administration.

In conclusion, the integrated view on the socio-technical system, including stakeholders, land administration processes and UAV technology as system elements, allows identifying interactions and positive and negative dependencies. Whereas the aspects of data quality and regulations both influence but are also impacted by other elements, land data needs were found to have a critical role in the system and predominantly affect other elements as a starting point for any land tool intervention. Representing the overlap of all three system elements, the diffusion of UAV technology in land administration takes a central place. Here, the superior quality of UAV data is a key driver throughout the diffusion process. In contrast, regulations may positively or negatively affect the emergence of UAV technology, as appropriate UAV legislation presents a vital pre-condition for technology uptake. Ultimately and potentially most importantly, land data needs stimulate the scaled implementation creating a demand for up-to-date high-resolution land data.

Overall, the findings of this thesis suggest that UAV technology is a highly flexible, widely available, easy-to-use and end-user responsive data acquisition tool that complements the range of mapping tools for land administration processes side by side with satellite data and GNSS surveying. Yet, regulatory constraints, missing awareness and weak governance support currently hamper exploiting the full potential of UAV technology for land administration in many countries. 


\section{Samenvatting}

Duidelijke grondeigendomsrechten bevorderen de zekerheid van grondbezit; een cruciale factor voor de bestrijding van honger en armoede, vrede, economische groei en het duurzaam gebruik van de omgeving. Een groot deel van de wereldbevolking voelt zich echter onzeker over haar eigendomsrechten, hetgeen vooral te wijten is aan het ontbreken van documentatie. In de afgelopen jaren zijn geospatiale innovaties en alternatieve concepten voor de registratie van grondbezit ontwikkeld. Één van deze innovaties betreft onbemande luchtvaartuigen (UAVs) die flexibele, goedkope en real-time verwerving van hoge resolutie luchtfoto's mogelijk maken. Deze kenmerken worden van cruciaal belang geacht voor de aanpak van de huidige uitdagingen op het gebied van land administratie.

Hoewel de gerapporteerde voordelen veelbelovend lijken, blijft de daadwerkelijke toepassing van UAVs als innovatief instrument voor kadastrale kartering opmerkelijk achter. Eerdere studies verwijzen voornamelijk naar data-gedreven en technologische voordelen van het gebruik van UAVs en houden geen rekening met maatschappelijke, bestuurlijke en institutionele omstandigheden en behoeften van mensen en groepen. Om deze leemte op te vullen wordt in dit proefschrift gebruik gemaakt van een sociaal-technologische benadering om UAV-gebaseerde gegevensinwinningsprocessen te onderzoeken als een instrument voor verantwoorde land administratie. Bovendien legt dit proefschrift de nadruk op de onderlinge relatie van drie belangrijke systeemelementen: technologie (UAVs), processen (land administratie processen) en actoren (stakeholders). Deze onderlinge relaties worden weerspiegeld in vier specifieke onderzoeksdoelstellingen. Ingebed in het H2020 gefinancierde internationale onderzoeks- en innovatieproject its4land, zijn delen van dit onderzoek uitgevoerd in de Oost-Afrikaanse context, inclusief testvluchten, workshops en karteringsactiviteiten.

Het eerste deel beoordeelt de mogelijkheden van UAV-technologie om aan de behoeften van landprofessionals in Rwanda te voldoen. Een evaluatie van de behoeften maakt het mogelijk om de behoeften aan landinformatie op verschillende niveaus en in verschillende sectoren van belanghebbenden in kaart te brengen. Hoewel niet steeds als eerste, werd de behoefte aan actuele landinformatie met hoge resolutie consequent vermeld in de bevindingen van alle groepsdiscussies. Testvluchten met meerdere UAVs en gegevensverwerking in Rwanda hebben aangetoond dat UAVs in staat zijn waardevolle ruimtelijke 
informatie te verschaffen voor diverse belanghebbenden. Uit het onderzoek in Rwanda, komen echter ook vier belangrijke uitdagingen naar voren voor UAV-inzet om landinformatie te genereren: 1) het heuvelachtige terrein, 2) UAV-regelgeving, 3) naverkenning in stedelijke gebieden, 4) gegevensverwerkings-capaciteit bij de nationale land administratie dienst. Kortom, hoewel UAVs een sleutelrol kunnen spelen bij het voldoen aan de behoeften van de belanghebbenden, vormen de structurele en capaciteitsbeperkingen momenteel grote uitdagingen voor het benutten van het potentieel van UAV-gegevensinwinningsprocessen.

Getriggerd door de enorme obstakels in de regelgeving voor het vliegen met UAVs in Oost-Afrika, analyseert het tweede deel van dit proefschrift de interface tussen UAV gegevensinwinning en belanghebbenden in termen van wet en regelgeving. Om een beter inzicht te krijgen in het verleden, het heden en de toekomstige ontwikkelingen van UAV-regelgeving, werd een grondige literatuurstudie en een vergelijkende analyse van nationale UAVregelgeving uitgevoerd. Hoewel alle UAV-regelgeving een vergelijkbaar doel heeft - het minimaliseren van het risico voor mensen en eigendommen op de grond - werd een sterk uiteenlopende aanpak in nationale regelgeving vastgesteld. Gemeenschappelijke kenmerken zoals verplichte platformregistratie, verplichte afsluiting van een verzekering en standaardprocedures voor pilotenvergunningen wijzen echter op trends in de richting van volwassen nationale UAVregelgeving. Het naast elkaar bestaan van harde en zachte regelgeving en de succesvolle internationale dialoog die uiteindelijk een juridisch kader voor geharmoniseerde regelgevingsnormen kan opleveren, zijn wenselijke ontwikkelingen.

Met als doel om optimale workflows aan te bevelen voor UAVgegevensinwinning onderzoekt het derde deel van dit proefschrift de technische en operationele aspecten van UAV-workflows en beoordeelt verschillende maatregelen met betrekking tot de datakwaliteit. Beeldmateriaal van zes studiegebieden in Europa en Afrika, die samen meer dan 100 verschillende vluchtconfiguraties vertegenwoordigen, vormen de basis voor een geïntegreerde beoordeling van de gegevenskwaliteit, inclusief verschillende aspecten van de fotogrammetrische verwerkingsketen. Uit de resultaten blijkt dat de context van ieder beeld, de vluchtconfiguratie en de GCP-opstelling aanzienlijke invloed hebben op de uiteindelijke gegevenskwaliteit en de daaropvolgende automatische detectie van visuele kadastrale grenzen. Hoewel de kwadratische gemiddelde fout van residuen van controlepunten in alle datasets binnen een bereik van enkele 
centimeters ligt, tonen onze resultaten grote verschillen in de nauwkeurigheid en de volledigheid van automatisch gedetecteerde kadastrale grenzen in orthofoto's gegenereerd uit verschillende vluchtplannen. Dankzij de unieke combinatie van methoden en de integratie van verschillende studie locaties kunnen deze resultaten en aanbevelingen zowel professionals als bottom-up initiatieven helpen om bestaande en toekomstige UAV-gegevensinwinningsprocessen te optimaliseren.

Ondanks de vooruitzichten en marktkansen op het gebied van land administratie, gaapt er een kloof tussen het experimenteren met, de verspreiding van en toepassing van UAV-technologie. Om de dynamiek van het verspreidingsproces van UAV-technologie als een instrument voor effectieve land administratie te begrijpen, integreert het vierde deel van dit proefschrift het Framework for Effective Land Administration (FELA) en het Hype Cycle-concept. De meeste deskundigen schatten in dat de UAV-technologie zich in een fase bevindt waarin de innovatie de initiële onvervulde verwachtingen moet overwinnen om marktontwikkeling en verdere aanvaarding te bevorderen. De beoordeling wijst op het veranderende belang van de verschillende FELA-trajecten tijdens dit proces, geleid door een afwisselende top-down- en bottom-up dynamiek. Wetgeving en beleid zijn, naast ondersteunende aansturing, verantwoordingsplicht en instituties, van cruciaal belang om in een vroeg stadium van het proces van invoering van de technologie een UAV-vriendelijk nationaal ecosysteem tot stand te brengen en te hoog gespannen verwachtingen weg te nemen. Zodra dit ecosysteem tot stand is gebracht, zal de marktvraag naar verwachting sterk toenemen onder invloed van samenwerkingsverbanden, aangepaste normen, technische belangenbehartiging en bewustmakingscampagnes, waarin de toegevoegde waarde van gegevens met hoge resolutie en andere voordelen van UAV-technologie worden belicht. Deze inzichten kunnen worden gebruikt als uitgangspunt om nationale strategische beslissingen te sturen in de richting van een toenemend gebruik van UAV's bij land administratie.

Concluderend kan worden gesteld dat de geïntegreerde visie op het socio-technische systeem, met belanghebbenden, land administratie processen en UAV-technologie als systeemelementen, het mogelijk maakt interacties en positieve en negatieve afhankelijkheden te identificeren. T De aspecten rondom datakwaliteit van UAV producten en de regelgeving inzake UAV vluchten beïnvloeden beide andere systeemelementen, maar worden daar zelf ook weer door beïnvloed. Nog belangrijker blijkt de door stakeholders ervaren behoefte aan 
landinformatie te zijn; deze vormt het startpunt bij de inzet van geospatiale innovaties, zoals UAVs, in de land administratie. De verspreiding van UAV-technologie in de land administratie, laat duidelijke de verwevenheid van de drie systeemelementen zien. In de eerste plaats is de superieure kwaliteit van UAV-gegevens een belangrijke drijfveer in het hele verspreidingsproces. In de tweede plaats kan de regelgeving een positieve of negatieve invloed hebben op de opkomst van de UAV-technologie, aangezien een passende UAVwetgeving een essentiële voorwaarde is voor de invoering van de technologie. In de derde en mogelijk belangrijkste plaats stimuleren de behoefte aan landinformatie de grootschalige inzet, waardoor er een vraag ontstaat naar actuele landgegevens met een hoge resolutie.

In het algemeen suggereren de bevindingen van dit proefschrift dat UAV-technologie een zeer flexibel, op grote schaal beschikbaar, gebruiksvriendelijk en op de behoeften van de eindgebruiker afgestemd instrument voor gegevensinwinning is, dat een aanvulling vormt op het scala van karteringsinstrumenten voor land administratie zoals satellietgegevens en GNSS-metingen. Toch belemmeren wettelijke beperkingen, een gebrek aan bewustzijn en een zwakke ondersteuning vanuit het bestuur momenteel de volledige benutting van het potentieel van UAV technologie voor land administratie in veel landen. 


\section{Zusammenfassung}

Verlässliche Landrechte fördern die Sicherheit von Grundbesitz - ein entscheidender Faktor für die Bekämpfung von Hunger und Armut, der Wahrung von Frieden, von Wirtschaftswachstum und der nachhaltigen Nutzung von Ressourcen. Ein Großteil der Weltbevölkerung fühlt sich jedoch unsicher in Bezug auf seine Landrechte, was hauptsächlich auf eine fehlende Dokumentation ebendieser zurückzuführen ist.

In den letzten Jahren wurden zunehmend raumbezogene Innovationen und alternative Konzepte für die Registrierung und Kartierung von Grundbesitz entwickelt. Eine dieser Innovationen umfasst unbemannte Luftfahrzeuge (UAVs), die eine flexible, kostengünstige und EchtzeitErfassung von hochauflösenden Luftbildern ermöglichen. Diese Funktionalitäten werden als entscheidend für die Bewältigung der aktuellen Herausforderungen der Landadministration angesehen.

Obwohl die berichteten Vorteile vielversprechend zu sein scheinen, wird der Einsatz von UAVs als innovatives Instrument für die Landvermessung nach wie vor kaum genutzt. Bisherige Arbeiten beziehen sich hauptsächlich auf die datenbezogenen und technologischen Vorteile des Einsatzes von UAVs und lassen die gesellschaftlichen, verwaltungstechnischen und institutionellen Rahmenbedingungen sowie Bedürfnisse diverser Akteure unberücksichtigt. Um diese Lücke zu schließen, wird in dieser Arbeit ein sozio-technischer Ansatz verwendet, um UAV-basierte Datenerfassungsworkflows als Instrument für eine verantwortungsvolle Landadministration zu untersuchen. Darüber hinaus wird in dieser Arbeit der Schwerpunkt auf die Wechselbeziehung zwischen drei Schlüsselelementen des sozio-technischen Systems gelegt: Technologie (UAVs), Prozesse (Landadministrationsprozesse) und Akteure (Interessengruppen).

Diese Zusammenhänge spiegeln sich in vier spezifischen Forschungszielen wider. Eingebettet in das von der EU im Rahmen des H2020-Programms finanzierte internationale Forschungs- und Innovationsprojekt its4land, wurden weite Teile dieser Arbeit im ostafrikanischen Kontext durchgeführt. Dies umfasste insbesondere diverse Testflüge, Workshops und Kartierungsaktivitäten.

Der erste Teil dieser Dissertation untersucht, inwieweit die UAVTechnologie den Bedürfnissen der Akteure der Landadministration in Ruanda entspricht. Eine Bedarfsanalyse ermöglichte es, eine Reihe von Landinformationsbedürfnissen in verschiedenen Sektoren der 
Landadministration zu ermitteln. Obwohl unterschiedlich eingestuft, wurde der Bedarf an hochauflösenden, aktuellen Landinformationen in den Ergebnislisten aller Gruppendiskussionen durchweg genannt. Testflüge mit verschiedenen UAVs und die Prozessierung eines Beispieldatensatzes in Ruanda haben gezeigt, dass es UAV Technologie vermag, wertvolle raumbezogene Informationen für verschiedene Interessengruppen zu liefern. Die Studie deutet jedoch auch auf vier Hauptaspekte hin, die Herausforderungen für die Nutzung von UAVs zur Gewinnung von Landinformationen im Kontext Ruandas aufzeigen können: 1) das hügelige Gelände, 2) UAV-Regularien, 3) die Erhebung von Bodenreferenzdaten in städtischen Gebieten, sowie 4) Kapazitäten für die Datenprozessierung an der nationalen Behörde für Landadministration. Zusammenfassend lässt sich sagen, dass UAVs zwar eine Schlüsselrolle bei der Erreichung der Bedürfnisse der Interessengruppen spielen können, die strukturellen und kapazitiven Bedingungen jedoch derzeit große Herausforderungen darstellen, um das Potenzial der UAV-Datenerfassungsabläufe optimal zu nutzen.

Ausgelöst durch die erheblichen regulatorischen Hindernisse des Einsatzes von UAVs in Ostafrika, analysiert der zweite Teil dieser Arbeit die Schnittstelle zwischen UAV-Technologie und Akteuren im Hinblick auf rechtliche Rahmenbedingungen des Einsatzes von UAVs. Um ein besseres Verständnis der vergangenen, gegenwärtigen und zukünftigen Entwicklungen der UAV-Regularien zu erhalten, wurde eine umfassende Literaturrecherche und eine vergleichende Analyse der nationalen UAV-Regularien durchgeführt. Obwohl alle UAVVorschriften ein ähnliches Ziel verfolgen - die Minimierung des Risikos, dass Menschen und Objekte am Boden zu Schaden kommen- wurde eine deutliche Heterogenität der nationalen Regelwerke ermittelt. Gemeinsamkeiten wie die obligatorische Registrierung von Luftfahrzeugen, der verpflichtende Versicherungsschutz und Standardverfahren für die Pilotenlizenzierung deuten jedoch auf einen Trend zu einer ausgereiften nationalen UAV-Regulierung hin. Wünschenswerte Entwicklungen werden in der Koexistenz harter und weicher Regelwerke und erfolgreicher internationaler Dialoge gesehen, welche letztendlich einen rechtlichen Rahmen für standardisierte Vorschriften schaffen könnten.

Der dritte Teil dieser Arbeit untersucht die technischen und operativen Aspekte von UAV-Datenerfassungsabläufen und bewertet verschiedene Aspekte der Datenqualität, mit dem Ziel, optimale UAV-Prozessabläufe zu empfehlen. Bildmaterial aus sechs Untersuchungsgebieten in Europa und Afrika, das insgesamt mehr als 100 verschiedene Flugkonfigurationen repräsentiert, bildete die Grundlage für eine 
integrierte Bewertung der Datenqualität unter Einbeziehung unterschiedlicher Gesichtspunkte der photogrammetrischen Prozessierung. Die Ergebnisse deuten darauf hin, dass der landschaftliche Kontext, die Flugkonfiguration und das Setup von Bodenkontrollpunkten einen erheblichen Einfluss auf die endgültige Datenqualität und die anschließende automatische Detektion von visuellen Katastergrenzen haben. Obwohl der mittlere quadratische Fehler der Kontrollpunktresiduen als allgemein akzeptiertes Fehlermaß in allen Datensätzen innerhalb eines Bereichs von wenigen Zentimetern liegt, zeigen unsere Ergebnisse große Diskrepanzen bei der Genauigkeit und Vollständigkeit der auf Orthophotos automatisch erkannten Bildobjekte. Durch die einzigartige Kombination von Methoden und die Integration verschiedener Untersuchungsstandorte können diese Ergebnisse und Empfehlungen sowohl Landexperten als auch Bottom-up-Initiativen dabei helfen, bestehende und künftige Arbeitsabläufe der UAV-Datenerfassung optimal auszurichten.

Trotz vielversprechender Aussichten und Vermarktungsmöglichkeiten in der Landadministration klafft eine Lücke zwischen der Erprobung und der weiten Verbreitung von UAV-Technologie. Um die Dynamik des Entwicklungsprozesses der UAV-Technologie als Instrument für eine effektive Landverwaltung zu verstehen, werden im vierten Teil dieser Arbeit das Framework for Effective Land Administration (FELA) und das Hype Cycle Konzept herangezogen. Die Mehrheit der Experten schätzt, dass sich die UAV-Technologie noch stets in einem Hype befindet. In dieser Phase gilt es, anfänglich unerfüllte Erwartungen zu überwinden, um im weiteren Verlauf die Marktentwicklung und eine verstärkte Akzeptanz zu fördern. Die Forschungsergebnisse weisen auf eine unterschiedliche Bedeutung verschiedener FELA-Pathways hin, welche durch eine alternierende Top-down- und Bottom-up-Dynamik geleitet werden. Es hat sich gezeigt, dass Gesetze und politische Maßnahmen sowie Verwaltungsstrukturen, Verantwortlichkeiten und Institutionen von entscheidender Bedeutung sind, um frühzeitig im Prozess der Einführung von UAVs ein förderliches Ökosystem zu schaffen und überzogene Erwartungen zu zerstreuen. Ist dieses Ökosystem etabliert, dürfte die Marktnachfrage durch Partnerschaften, angepasste Standards, technische Lobbyarbeit und Sensibilisierungskampagnen stark ansteigen. Diese Erkenntnisse können als Grundlage für nationale strategische Entscheidungen herangezogen werden, um den Einsatz von UAVs in der Landadministration zu stärken.

Zusammenfassend lässt sich sagen, dass die integrierte Betrachtung des sozio-technischen Systems, welche die Akteure, die Landverwaltungsprozesse und die UAV-Technologie als 
Systemelemente einschließt, es ermöglicht, Wechselwirkungen sowie positive und negative Abhängigkeiten zu erkennen. Während die Aspekte der Datenqualität und der UAV-Regulationen sowohl Einfluss auf andere Elemente haben als auch von diesen beeinflusst werden, wurde festgestellt, dass die Ansprüche an Grundbesitzinformationen eine entscheidende Rolle im System spielen. Diese beeinflussen in erster Linie die anderen Systemelemente, da jene Ansprüche den Ausgangspunkt für jede Intervention im Bereich der Landadministration bilden. Wird die Überschneidung aller drei Systemelemente betrachtet, kommt der Verbreitung der UAVTechnologie in der Landadministration eine zentrale Rolle zu. Zunächst ist die überlegene Qualität der UAV-Daten eine wichtige Triebkraft des gesamten Verbreitungsprozesses. Dagegen können sich Rechtsvorschriften positiv oder negativ auf das Aufkommen von UAVTechnologie auswirken, da eine angemessene UAV-Gesetzgebung eine wesentliche Voraussetzung für den weiteren Innovationsprozess darstellt. Zusätzlich spielt auch der Bedarf an Landinformationen eine wichtige Rolle, da somit die skalierte Implementation angeregt und eine gesteigerte Nachfrage nach aktuellen, hochauflösenden Landdaten geschaffen werden kann.

Insgesamt deuten die Ergebnisse dieser Arbeit darauf hin, dass UAVTechnologie ein äußerst flexibles, weithin verfügbares, einfach zu verwendendes und auf die Bedürfnisse der Endnutzer abgestimmtes Instrument zur Datenerfassung ist, welches die Palette der Kartierungsinstrumente für Landadministrationsprozesse neben Satellitendaten und GNSS-Vermessungen ergänzt. Dennoch behindern regulatorische Beschränkungen, fehlendes Bewusstsein und schwache Governance derzeit die Nutzung des vollen Potenzials von UAVTechnologie für Landadministration in vielen Ländern. 


\section{Author's Biography}

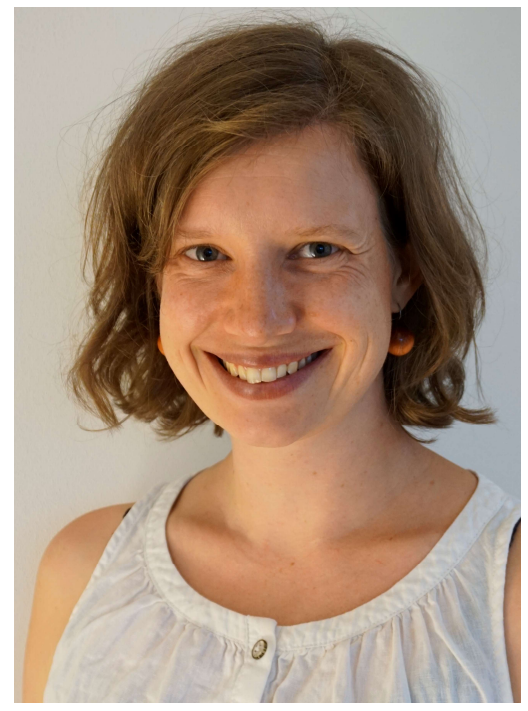

Claudia was born on January $19^{\text {th }}$ 1989, in Dresden (Germany). She received a Bachelor degree in Geography in 2011 from the Dresden University of Technology. Afterwards, she pursued a Master course in Global Change - Regional Sustainability and graduated with a Master of Science from the University of Innsbruck in 2014. She started working with unmanned aerial vehicles (UAVs) and UAV data, first as a student assistant in an Austrian company and later as a researcher at the Institute of Photogrammetry at the Dresden University of Technology.

Fascinated by the almost unlimited capabilities of UAVs for data capture, Claudia started as a PhD candidate at the University of Twente, Faculty of Geo-information Science and Earth Observation in 2016 - which has resulted in the present manuscript. As a PhD candidate, she was involved in teaching and supervision of MSc students. In 2020, Claudia received the SARES young researcher award from the International Sustainable Aviation and Energy Research Society. During her research, Claudia authored and coauthored various papers in remote sensing and land administration journals. In addition, her work has been presented at numerous national and international conferences, including FIG conferences, ISPRS conferences and the annual World Bank conference in Washington.

Besides her PhD research, Claudia was involved in the organisation of the Lake Kivu Challenge at the African Drone Forum 2020. Since 2017, she has been part of the editorial team of the book "UAVs in the environmental sciences", which will be published by the end of 2021. Besides her role as an editor, she authored and co-authored two chapters. In addition to this engagement, she acts as an active reviewer for leading remote sensing and land administration journals.

Claudia likes to be in nature and enjoys climbing, cycling, and hiking with her family in her leisure time. 


\section{Journal publications}

Stöcker, C., Bennett, R., Nex, F., Gerke, M., Zevenbergen, J. (2017): Review of the current state of UAV regulations. In : Remote sensing 9, 5, 459.

Stöcker, C., Ho, S., Nkerabigwi, P., Schmidt, C.Koeva, M., Bennett, R., Zevenbergen, J. (2019): Unmanned Aerial System Imagery, Land Data and User Needs: A Socio-Technical Assessment in Rwanda. In: Remote sensing 11, 9, 1035.

Stöcker, C., Nex, F., Koeva, M., Gerke, M. (2020): High-quality UAVbased orthophotos for cadastral mapping: Guidance for optimal flight configurations. In : Remote sensing 12, 21, 3625.

Stöcker, C., Bennett, R., Koeva, M., Nex, F., Zevenbergen, J.: Scaling up UAVs for land administration: towards the plateau of productivity. In: Land Use Policy (Undergoing minor revision).

Flores, C. C., Tan, E., Buntinx, I., Crompvoets, J., Stöcker, C., Zevenbergen, J. (2020): Governance assessment of the UAVs implementation in Rwanda under the fit-for-purpose land administration approach. In: Land Use Policy, 99, 104725.

Koeva, M., Stöcker, C., Crommelinck, S., Ho, S., Chipofya, M., Sahib, J., Bennett, R., Zevenbergen, J., Vosselman, G., Lemmen, C., Crompvoets, J., Buntinx, I., Wayumba, G., Wayumba, R., Odwe, P.O., Osewe, G.T., Chika, B., Pattyn, V. (2020): Innovative Remote Sensing Methodologies for Kenyan Land Tenure Mapping. In: Remote Sensing. 12(2):273.

Koeva, M., Humayun, M., Timm, C., Stöcker, C., Crommelinck, C., Chipofya, M., Bennett, R., Zevenbergen J. (2021): Geospatial Tool and Geocloud Platform Innovations: A Fit-for-Purpose Land Administration Assessment. In: Land 10, 6, 557.

\section{Conference papers and presentations}

Stöcker, C., Nex, F., Koeva, M., Gerke, M. (2017): Quality assessment of combined IMU/GNSS data for direct georeferencing in the context of UAV-based mapping. In: ISPRS 2017: International Conference on Unmanned Aerial Vehicles in Geomatics ISPRS Archives XLII-2/W6.

Stöcker, C., Nex, F., Koeva, M., Bennett, R., Zevenbergen, J. (2017): Conference presentation: Towards UAV-based Land Tenure Data Acquisition. NCG Symposium. Delft. 
Koeva, M., Bennett, R., Gerke, M., Crommelinck, S., Stöcker, C., Crompvoets, J., Ho, S., Schwering, A., Chipofya, M., Schultz, C., Zein, T., Biraro, M., Alemie, B. K., Wayumba, R., Kundert, K. (2017): Towards innovative geospatial tools for fit-forpurpose land rights mapping. In: Proceedings ISPRS geospatial week 2017 ISPRS Vol. XLII-2/W7

Bennett, R., Gerke, M., Crompvoets, J., Alemie, B. K., Crommelinck, S., Stöcker, C. (2017): Building Third Generation Land Tools: Its4land, Smart Sketchmaps, UAVs, Automatic Feature Extraction, and the GeoCloud. In: Proceedings of the Annual World Bank Conference on Land and Poverty The World Bank 2017.

Stöcker, C., Ho, S., Koeva, M., Nkerabigwi, P., Schmidt, C., Zevenbergen, J., Bennett, R. (2018): Towards UAV-based Land Tenure Data Acquisition in Rwanda: Needs Assessment and Technology Response. In: XXVI FIG Congress 2018: Embracing our smart world where the continents connect: enhancing the geospatial maturity of societies (FIG)

Stöcker, C., Koeva, M., Zevenbergen, J. (2018): Conference presentation: UAV Technology: Opportunities and Limitations to support Land Administration. LANDac International Conference 2018: Land Governance and (Im)mobility.

Stöcker, C., Nex, F., Koeva, M., Zevenbergen, (2018): Data quality assessment of UAV-based products for land tenure recording in East Africa. In: NCG Symposium, Wageningen.

Koeva, M. N., Crommelinck, S., Stöcker, C., Crompvoets, J. (2018): Its4land - Challenges and Opportunities in Developing Innovative Geospatial Tools for Fit-For-Purpose Land Rights Mapping. In: XXVI FIG Congress 2018: Embracing our smart world where the continents connect: enhancing the geospatial maturity of societies (FIG)

Gerke, M., Stöcker, C., Crommelinck, S., Koeva, M. (2018): UAV für das Kadaster - das EU-Projekt its4land. In : UAV 2018 Vermessung mit unbemannten Flugsystemen: Beiträge zum 169. DVW-Seminar Deutsche Gesellschaft fur Geodesie, Geoinformation, und Landmanagement, p. 10512 p. 116.

Ho, S., Chipofya, M., Stöcker, C., Crommelinck, S, Timm, C. (2018): Smart Sustainable Futures in Land Administration: A Role for Emerging Technologies. In: International Symposium on a Smart Sustainable Future for All - Enhancing Resilience in a Changing Landscape. University of Melbourne, Australia. 
Stöcker, C., Nex, F., Koeva, M., Gerke, M. (2019): UAV-based cadastral mapping: an assessment of the impact of flight parameters and ground truth measurements on the absolute accuracy of derived orthoimages. In : The International Archives of the Photogrammetry, Remote Sensing and Spatial Information Sciences: ISPRS Geospatial Week 2019 ISPRS Archives, Vol. XLII-2/W13.

Stöcker, C., Koeva, M., Zevenbergen, J. (2019): UAV Technology: Opportunities to support the updating process of the Rwandan cadastre. In: Annual Conference of EALAN - East African Land Administration Network, Zanzibar.

Stöcker, C., Koeva, M., Zevenbergen, J., Bennett, R. (2019): UAVbased cadastral mapping: Evaluation of UAV-based technology to capture land rights in Kenya: displaying stakeholder perspectives through interactive gaming. In: 20th Annual World Bank Conference on Land and Poverty 2019: Catalyzing Innovation - Washington DC, United States The World Bank.

Stöcker, C. Koeva, M., Nkerabigwi, P., Zevenbergen, J. (2020): UAV Technology: Opportunities to support the updating process of the Rwandan cadastre. In: FIG Working Week - Smart surveyors for land and water management (FIG).

Stöcker, C. (2020): ICUAV - International Course on Unmanned Aerial Vehicles, Kyiv, Invited speaker: UAV regulations - Current state and past developments.

\section{Data repository}

All data used for this research has been published through Data Archiving and Networked Services (DANS) on behalf of the Royal Netherlands Academy of Arts and Sciences.

Stöcker, C (Faculty of Geo-Information Science and Earth Observation (ITC), University of Twente) (2020): its4land UAV data acquisition workflows. DANS. https://doi.org/10.17026/dansxhz-uqct. 